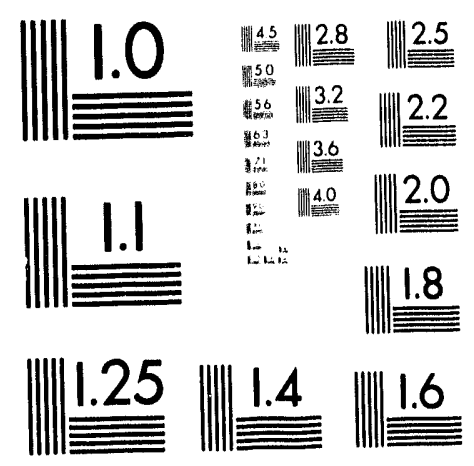



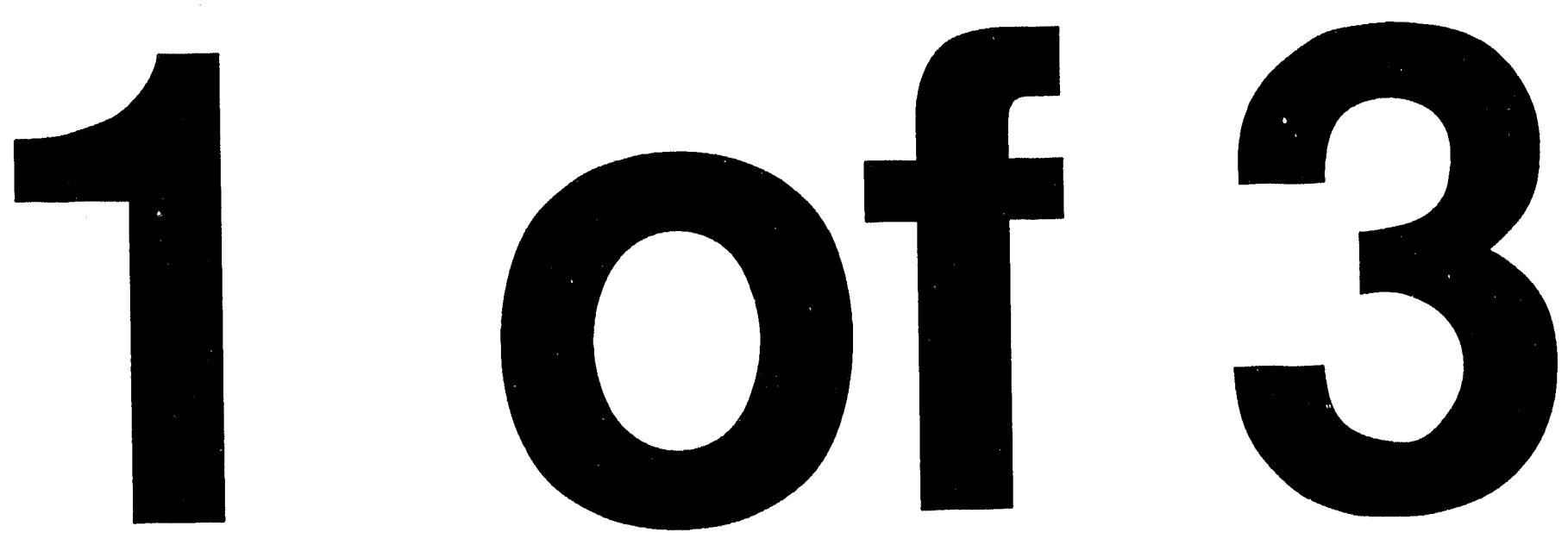
LBL-34481

UC-401

\title{
HIGH-RESOLUTION SOFT X-RAY PHOTOIONIZATION STUDIES OF SELECTED MOLECULES
}

\author{
Eric Allen Hudson \\ $\mathrm{Ph} . \mathrm{D}$. Thesis \\ DEPARTMENT OF CHEMISTRY \\ University of California \\ and \\ CHEMICAL SCIENCES DIVISION \\ Lawrence Berkeley Laboratory \\ University of California \\ Berkeley, CA 94720
}

AUGUST 1993

This work was supported by the Director, Office of Energy Research, Office of Basic Energy Sciences, Chemical Sciences Division, of the U.S. Department of Energy, under Contract No. DE-AC03-76SF00098. 


\begin{abstract}
by

Eric Allen Hudson

Doctor of Philosophy in Chemistry

University of California at Berkeley

Professor David A. Shirley, Chair
\end{abstract}

High-Resolution Soft X-Ray Photoionization Studies Of Selected Molecules

Near-edge soft $\mathrm{x}$-ray photoionization spectra were measured with high resolution in energy for a series of molecules in the gas phase, using the Free University of Berlin plane-grating SX-700-II monochromator at the synchrotron radiation source BESSY. Analysis of the spectra provides details of the core-excited electronic states which are accessed, including quantum defects, natural linewidths, vibrational spacings, molecular geometries, and molecular-field splittings. The interpretation of these results characterizes the valence and Rydberg orbitals which are populated by the transitions, as well as the core orbitals which are vacated.

Photoionization spectra of carbon monoxide were measured near the carbon and oxygen $\mathrm{K}$ edges. Vibrational spacings and bond lengths are derived for several resonances. Results are consistent with the equivalent-core model and indicate the different influences of the carbon and oxygen 1 s core holes. Corresponding spectra of $\mathrm{H}_{2} \mathrm{CO}$ and $\mathrm{D}_{2} \mathrm{CO}$ were also measured. Assignment of the complex vibrational structure in the valence-shell and Rydberg resonances is facilitated by a comparison of the spectra for the two isotopic species. Geometric and vibrational parameters are derived for several carbon $1 s$ core-excited states. Isotopic shifts are observed in the energies and linewidths of some core-excited states.

Sulfur hexafluoride photoionization spectra, measured near the sulfur $\mathrm{L}_{2,3}$ edges, show several series of weak, narrow Rydberg resonances. The high resolution and good 
counting statistics allow a complete assignment of these states. The lineshapes of the broad inner-well resonances also observed in these spectra are analyzed to establish the magnitudes of vibrational and lifetime broadening in these states.

Spectra of the $\mathrm{H}_{2} \mathrm{~S}$ and $\mathrm{D}_{2} \mathrm{~S}$ molecules were also measured near the sulfur $\mathrm{L}_{2,3}$ edges. Besides lower-energy transitions to inner-well states, a complex manifold of overlapping Rydberg resonances is observed. The rich fine structure of these states arises mainly from the removal of orbital degeneracies in the molecular field. Additional structure due to vibrational excitations in the final state is identified by a comparison of the spectra for the two isotopic species. 


\section{TABLE OF CONTENTS}

List Of Figures............................................................ iv

List $O f$ Tables.................................................................. vi

List of Related Publications...................................................... vii

Dedication..................................................................... viii

Acknowled gements.......................................................... ix

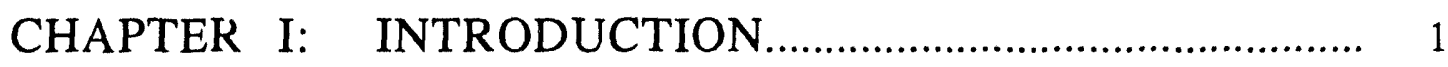

A. Motivations: What may be learned from these experiments?......... 1

B. Historical development of soft x-ray absorption spectroscopy....... 4

C. Survey of present measurements..................................... 6

D. Basic principle and common themes ............................... 7

1. Rydberg states........................................... 8

2. Valence-shell states........................................ 10

3. Intermediate or mixed states.................................. 11

4. Vibrational structure................................... 12

5. Equivalent-core approximation............................. 14

6. Dissociative states and zero-point vibrational energy.......... 15

7. Core-hole spin-orbit interaction............................. 17

E. Organization of chapters...................................... 18

References.................................................... 19

CHAPTER II: EXPERIMENT AND DATA ANALYSIS............... 21

A. Synchrotron Radiation....................................... 21

B. The SX700/II monochromator................................. 21

C. Photoionization gas cell........................................... 22

D. Data analysis............................................. 25

References......................................................... 27

Figures............................................................ 28

\section{CHAPTER III: HIGH-RESOLUTION PHOTOIONIZATION} OF CARBON MONOXIDE AT THE CARBON AND OXYGEN $\mathrm{K}-\mathrm{EDGES}$

A. Introduction.................................................. 31

B. Experimental.................................................... 31

C. Carbon K-edge................................................ 32

1. Overview......................................... 32

2. $\quad(\mathrm{C} 1 \mathrm{~s})^{-1} \pi^{*}$ resonance................................... 32

3. $\quad(\mathrm{C} 1 \mathrm{~s})^{-1}$ Rydberg resonances.............................. 33

D. Oxygen K-edge.................................................. 34

1. Overview................................................ 34

2. $(\mathrm{O} 1 \mathrm{~s})^{-1} \pi^{*}$ resonance.................................. 35 
3. $\quad(\mathrm{O} 1 \mathrm{~s})^{-1}$ Rydberg resonances................................. 36

E. Comparison to equivalent-core molecules.............................. 36

References......................................................... 38

Tables.................................................................. 39

Figures................................................................ 41

\section{CHAPTER IV: HIGH-RESOLUTION PHOTOIONIZATION OF FORMALDEHYDE AT THE CARBON AND OXYGEN K-ED GES .................................................................... 46}

A. Introduction.................................................... 46

B. Experimental............................................... 47

C. Carbon K-edge................................................ 47

1. Overview..................................................... 47

2. (C $1 \mathrm{~s})^{-1} \pi^{*}$ resonance....................................... 48

3. (C 1s) ${ }^{-1}$ Rydberg resonances................................. 50

4. Franck-Condon analysis................................... 54

D. Oxygen K-edge................................................. 59

E. Isotopic effects............................................. 61

1. Isotopic effects on vibrational fine structure.................. 61

2. Isotopic effects on the (C 1s) $)^{-1} 3 s-a_{1}$ and $\left(\mathrm{O}_{1}\right)^{-1} 3 s-a_{1}$ Rydberg states..................................... 63

3. Comparison to the $\left(2 b_{1}\right)^{-1}$ valence-excited Rydberg states of formaldehyde................................... 66

4. Similar isotopic effects in the $(\mathrm{C} 1 \mathrm{~s})^{-1} 3 \mathrm{~s}$ states of other molecules..................................... 67

5. An alternative explanation for the isotopic effects on the

References. $(\mathrm{C} 1 \mathrm{~s})^{-1} 3 \mathrm{~s}-\mathrm{a}_{1}$ and $(\mathrm{O} 1 \mathrm{~s})^{-1} 3 \mathrm{~s}-\mathrm{a}_{1}$ Rydberg states.... 68

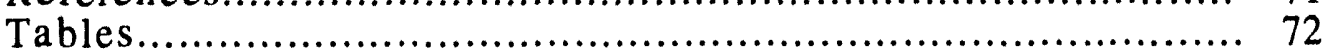

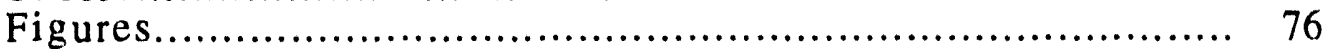

\section{CHAPTER V: HIGH-RESOLUTION MEASUREMENTS OF NEAR-EDGE RESONANCES IN THE CORE-LEVEL PHOTOIONIZATION SPECTRA OF $\mathrm{SF}_{6}$.}

A. Introduction...................................................... 83

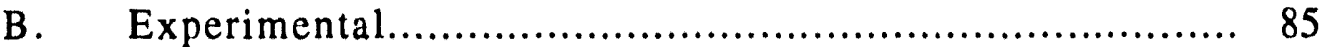

C. Overview of results............................................. 85

D. Sulfur $L_{2,3}$ thresholds: Inner-well resonances......................... 87

1. Spin-orbit interaction and exchange interaction................. 87

2. Lineshapes............................................... 88

a. $\quad(\mathrm{S} \mathrm{2p})^{-1}$ a $1 \mathrm{~g}$ resonances............................ 88

b. $\quad(\mathrm{S} 2 \mathrm{p})^{-1} \mathrm{t}_{2 \mathrm{~g}}$ resonances.............................. 92

3. Vibronic coupling.......................................... 94

E. Sulfur $\mathrm{L}_{2,3}$ thresholds: Rydberg series.................................. 96

1. Analysis using the Rydberg formula............................ 96

2. Multiplet splitting.............................................. 98 
3. Linewidths of the Rydberg resonances..................... 100

4. Vibrational structure.................................... 101

5. Sulfur np Rydberg orbitals................................... 104

6. Energies and edge jumps of the sulfur $L_{2,3}$ thresholds....... 106

F. Sulfur $\mathrm{L}_{2,3}$ thresholds: Comparison with theory.................... 106

G. Fluorine $\mathrm{K}$ threshold.......................................... 108

References........................................................... 110

Tables................................................................ 113

Figures........................................................... 117

\section{CHAPTER VI: HIGH-RESOLUTION PHOTOIONIZATION} AT THE SULFUR $\mathrm{L}_{1,2,3}$ THRESHOLDS: $\mathrm{H}_{2} \mathrm{~S}$ AND $\mathrm{D}_{2} \mathrm{~S} \ldots \ldots \ldots \ldots \ldots . .127$

A. Introduction .................................................. 127

B. Experimental............................................. 130

C. Overview of results.............................................. 131

D. Sulfur $L_{2,3}$ Edges: Non-degeneracy of the sulfur $2 p$ core level..... 132

E. Sulfur L2,3 Edges: Rydberg and Mixed Excitations.................... 135

1. General Considerations...................................... 135

2. Upper Range: Rydberg states............................... 138

3. Lower range: Rydberg and mixed states....................... 140

a. Approach used for analysis.......................... 140

b. Results of analysis.................................. 143

4. Comparison to other XANES spectra......................... 147

a. $\quad$ Mixed states.............................................. 147

b. Rydberg states.................................... 149

5. Comparison to previous $\mathrm{H}_{2} \mathrm{~S}$ results......................... 151

6. Vibrational structure........................................ 152

F. Sulfur L2,3 Edges: Valence Shell Excitations............................ 153

1. Assignment and electron-hole interaction...................... 153

2. Vibrational structure and other isotopic shifts................... 154

G. Linewidths............................................... 158

H. Sulfur $L_{1}$ Edge............................................... 159

I. Comparison to Theoretical Results.................................... 160

J. Conclusions................................................... 161

References........................................................ 163

Tables.............................................................. 166

Figures................................................................. 170

\section{CHAPTER VII: FUTURE DIRECTIONS.................................. 177}

A. Improvements in the present spectra................................. 177

B. High-resolution spectra of other molecules............................ 178

C. Further studies of core-excited electronic states using high-resolution

$\mathrm{x}$-rays................................................ 178

1. Photoelectron spectroscopy.................................... 179

2. Resonance autoionization electron spectroscopy............... 180

3. Photo-fragmentation spectroscopy.............................. 181

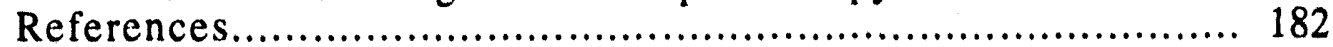




\section{LIST OF FIGURES}

Figure II-1: (Bottom) Schematic view of the SX700/II monochromator. (Top) The monochromator resolution $\triangle \mathrm{E}$ (FWHM) as a function of photon energy.

Figure II-2: Schematic view of the ionization cell used for the measurement of gas-phase total-ion-yield spectra.

Figure III-1: The photoabsorption spectrum of gas-phase $\mathrm{CO}$ near the carbon $\mathrm{K}$ edge.

Figure III-2: An expanded view of the Rydberg region of Fig. III-1, showing the (C 1s) $)^{-1}$ Rydberg states of CO.

Figure III-3: Spatial distribution of the $1 \pi$ molecular orbital in ground-state $\mathrm{CO}$ and NO.

Figure III-4: The photoabsorption spectrum of $\mathrm{CO}$ near the oxygen K-edge.

Figure IV-1: Overview of the photoionization spectrum of $\mathrm{H}_{2} \mathrm{CO}$ near the carbon $1 \mathrm{~s}$ ionization threshold IP C.K.

Figure IV-2: High-resolution spectrum of the (C $1 \mathrm{~s})^{-1} \pi^{*}$ resonance in (a) $\mathrm{H}_{2} \mathrm{CO}$ and (b) $\mathrm{D}_{2} \mathrm{CO}$.

Figure IV-3: Core-excited Rydberg states below the carbon K-edge in (a) $\mathrm{H}_{2} \mathrm{CO}$ and (b) $\mathrm{D}_{2} \mathrm{CO}$.

Figure IV-4: Spatial distributions of the molecular orbitals in ground-state $\mathrm{H}_{2} \mathrm{CO}$.

Figure IV-5: Photoionization spectrum of formaldehyde near the oxygen $1 \mathrm{~s}$ ionization threshold.

Figure V-1. Schematic representation of a double-well potential (not to scale).

Figure V-2. Photoabsorption spectrum of $\mathrm{SF}_{6}$ near the sulfur $\mathrm{L}_{2,3}$ edges.

Figure V-3. The $(S 2 p)^{-1} a_{1 g}$ resonances of $S_{6}$.

Figure V-4. Sulfur $\mathrm{L}_{2,3}$ pre-edge fine structure of the $\mathrm{SF}_{6}$ photoabsorption spectrum.

Figure V-5. Photoabsorption spectrum of $\mathrm{SF}_{6}$ near the fluorine $\mathrm{K}$ edge.

Figure V-6. Level diagram of the core-excited states of $\mathrm{SF}_{6}$ at the sulfur $\mathrm{L}_{2,3}$ and fluorine $\mathrm{K}$ edges.

Figure V-7. Franck Condon analysis of the vibrational structure of the (S 2p $)^{-1} 4 s$ state (peaks A,B,C in Fig. V-4). 
Figure VI-1. Overview of the photoionization spectrum of $\mathrm{H}_{2} \mathrm{~S}$ near the sulfur $\mathrm{L}$ edges.

Figure VI-2. High-resolution photoionization spectra of $\mathrm{H}_{2} \mathrm{~S}$ and $\mathrm{D}_{2} \mathrm{~S}$ in the mixed and Rydberg region of the sulfur $\mathrm{L}_{2,3}$ edges.

Figure VI-3. High-resolution photoionization spectra of $\mathrm{H}_{2} \mathrm{~S}$ and $\mathrm{D}_{2} \mathrm{~S}$ in the region of valence shell excitations below the sulfur $\mathrm{L}_{2,3}$ edges.

Figure VI-4. High-resolution photoionization spectra of $\mathrm{H}_{2} \mathrm{~S}$ and $\mathrm{D}_{2} \mathrm{~S}$ near the sulfur $\mathrm{L}_{1}$ edge.

Figure VI-5. Alternative fits to the data of Fig. VI-2, assuming there is no molecular-field splitting of the sulfur $2 \mathrm{p}_{3} / 2$ core level.

Note: Figures III-3 and IV-4 are used with the permission of Academic Press. Those figures may not be reproduced or transmitted in any form or by any means, aside from reproduction of this dissertation, without permission in writing from Academic Press. 


\section{LIST OF TABLES}

Table III-1: Energies and assignments of the $\mathrm{CO}(\mathrm{C} 1 \mathrm{~s})^{-1}$ core-excited states and their vibrational sidebands.

Tabie III-2: Equilibrium distances R, vibrational energies hv, and anharmonicity constants hvx for $\mathrm{CO}$ and $\mathrm{N}_{2}$ in the ground state and in core-excited states.

Table IV-1: Energies (in eV) of the (C $1 \mathrm{~s})^{-1}$ and $(\mathrm{O} 1 \mathrm{~s})^{-1}$ core-excited states of $\mathrm{H}_{2} \mathrm{CO}$ in comparison with previous EELS results and theoretical predictions.

Table IV-2: Contributions, in percent, of the symmetry coordinates to the normal modes in the ground states of $\mathrm{H}_{2} \mathrm{CO}$ and $\mathrm{D}_{2} \mathrm{CO}$.

Table IV-3: Derived equilibrium values for the $\mathrm{C}-\mathrm{O}$ and $\mathrm{C}-\mathrm{H}$ (C-D) bond lengths, $\mathrm{R}$ (in angstroms), and the $\mathrm{HCH}$ (DCD) molecular bond angle $\theta$, and vibrational energies $h v$ (in $\mathrm{meV}$ ) for the $v_{1}, v_{2}$, and $v_{3}$ modes in the ground and core-excited states of $\mathrm{H}_{2} \mathrm{CO}$ and $\mathrm{D}_{2} \mathrm{CO}$.

Table IV-4: Isotopic effects on energies $E$ and linewidths W (FWHM) of the (C 1s) $)^{-1}$ $3 \mathrm{~s}-\mathrm{a}_{1}$ and $(\mathrm{O} 1 \mathrm{~s})^{-1} 3 \mathrm{~s}-\mathrm{a}_{1}$ states of formaldehyde.

Table V-1 : $\quad \mathrm{SF}_{6}$ sulfur $2 p$ inner-well resonances: Assignments and fit results.

Table V-2: $\quad$ SF$_{6}$ sulfur $2 p$ Rydberg states: Assignments and fit results.

Table V-3 : $\quad \mathrm{SF}_{6}$ fluorine $\mathrm{K}$ inner-well resonances: Assignments and fit results.

Table V-4: Comparison of measured and calculated term values for the (S $2 p)^{-1}$ belowedge resonances in $\mathrm{SF}_{6}$.

Table VI-1 : $\quad \mathrm{H}_{2} \mathrm{~S}$ and $\mathrm{D}_{2} \mathrm{~S}(2 \mathrm{p})^{-1}$ Rydberg resonances: Assignments and fit results.

Table VI-2: $\quad \mathrm{H}_{2} \mathrm{~S}$ and $\mathrm{D}_{2} \mathrm{~S}(2 \mathrm{p})^{-1}$ mixed resonances: Fit results and vibrational assignments.

Table VI-3 : Quantum defects for Rydberg states in the L2,3 XANES spectra of Ar and the second-row hydrides.

Table VI-4: Results of least-squares analysis of the $\mathrm{H}_{2} \mathrm{~S}$ and $\mathrm{D}_{2} \mathrm{~S}(2 \mathrm{p})^{-1}$ valence-shell region: Approximate fits of overall lineshapes.

Table VI-5: Results of least-squares analysis of the $\mathrm{H}_{2} \mathrm{~S}$ and $\mathrm{D}_{2} \mathrm{~S}(2 \mathrm{p})^{-1}$ valence-shell region: Exact fit of fine structure. 


\section{LIST OF RELATED PUBLICATIONS}

1. "Carbon and Oxygen K-Edge Photoionizaton of the CO Molecule" M. Domke, C. Xue, A. Puschmann, T. Mandel, E. Hudson, D. A. Shirley, and G. Kaindl.

Chem. Phys. Lett. 173, 122 (1990); ibid, 174, 668 (1990).

Includes some of the research presented in Chapter III.

2. "High-Resolution K-Shell Photoabsorption in Formaldehyde"

G. Remmers, M. Domke, A. Puschmann, T. Mandel, C. Xue, G. Kaindl, E. Hudson, and D. A. Shirley.

Phys. Rev. A 46, 3935 (1992).

Includes some of the research presented in Chapter IV.

3. "High-Resolution Measurements of Near-Edge Resonances in the Core-Level Photoionization Spectra of $\mathrm{SF}_{6} "$

E. Hudson, D. A. Shirley, M. Domke, G. Remmers, A. Puschmann, T. Mandel, C. Xue, and G. Kaindl.

Phys. Rev. A 47, 361 (1993).

Includes some of the research presented in Chapter $\mathrm{V}$.

4. "High-Resolution Photoabsorption Near the Sulfur $\mathrm{l}_{-2,3}$ Thresholds: $\mathrm{H}_{2} \mathrm{~S}$ and $\mathrm{D}_{2} \mathrm{~S}$ " E. Hudson, D.A. Shirley, M. Domke, G. Remmers, and G. Kaindl.

Phys. Rev. A, submitted (1993).

Includes some of the research presented in Chapter VI. 
viii

This dissertation is dedicated to my parents, for their consistent love, encouragement, and support. 


\section{ACKNOWLEDGEMENTS}

\section{"Once in while you get shown the light \\ In the strangest of places if you look at it right." \\ -- Robert Hunter}

In the last six years, many people have provided me with guidance, advice, assistance, and support. First and foremost I must thank Dave Shirley, my advisor, for teaching me so much about science and research. He has shared his extensive knowledge and deep insight while allowing me to pursue the directions which interested me the most. I have had the additional good fortune to work very closely with another skilled scientist, Zahid Hussain. His enthusiasm for research is infectious and he has the rare talents needed to make difficult experiments succeed. The Shirley research group has provided a consistently stimulating intellectual environment as well as an enjoyable workplace. I thank Alexis, Jane, Lai-Sheng, Lou, Li-Qiong, Phil, and Zheng-qing for patiently answering countless questions, especially near the beginning of my studies. Baohua, Bear, Eddie, Scot, Tobias, Tony, and Yu all deserve credit for their support and for facing many challenges with good humor. I have benefited from the ideas and advice of many others in Berkeley and beyond, including Chuck Fadley, Norm, Jerry, Heino, David Shuh, Trish, Dennis, Marjorie, and members of the Neumark group. The staff of LBL is acknowledged for valuable technical support.

The experimental work in this dissertation was performed in Berlin, mostly during my ten-month visit in 1989-1990. After my return, I continued to work with these colleagues from afar. Gunter Kaindl provided a productive environment during my visit and I have learned niuch from our collaboration. Michael Domke deserves special thanks for his tireless efforts to make the monochromator the best possible and for his commitment. to the highest standards for our publications, despite the difficulties of a long-distance collaboration. I am indebted to the staff of BESSY and the members of the Kaindl group, 
especially Ching, Guido, Thomas Mandel, Alfred, Thomas Simmons, and Clemens. Klaus Baberschke and his group were also helpful, in particular I thank Dimitri, Hans, and Thomas.

Although they do not appear in this dissertation, I have in fact been involved with numerous experiments at SSRL. I must give credit to the staff of SSRL, especially the duty operators Don, Ken, John, Peter, and Joe, as well as Michelle, Tony, Brian, Tracy, George, Tom, and Mike. Their support makes SSRL a better place to work.

The work contained in this dissertation was supported by the Director, Office of Basic Energy Sciences, Chemical Sciences Division of the U. S. Department of Energy, under Contract No. DE-AC03-76SF00098 and the Bundesminister für Forschung und Technologie, Project No. 05-413AXI-7/TP4. I was partially supported by a University of California Regents Fellowship for two years of my studies.

For personal support I must acknowledge family members: my parents, my sister, Nana, and Rosemary and her clan. Many friends have helped to keep me going these last six years. I can only list some of them here: Ted, Batch, John, Beth, Andy, Mark, Paige, Jane, Eddie, Carina, Mike, Kathy, Stefano, Zack, Sue, Doug, Byrnes, Charlie, Rick, Deb, Steph, Lynn, Lui, Linda, Mark, and Jackie. Special thanks go to Jerry Garcia for his continuing efforts. Most of all, I thank Natasha for all she has shared--I couldn't have finished this work without her. 


\section{CHAPTER I}

\section{I.A. Motivations: What may be learned from these experiments?}

This dissertation presents and interprets the results of high-resolution soft $\mathrm{x}$-ray photoionization measurements. In particular, near-edge photoionization spectra were measured for a series of molecules in the gas phase. In this type of experiment, the total photoion current produced by a gas is measured as the photon energy is scanned through an energy range which includes the ionization threshold for a core-level electron of an atom in the molecule. Peaks are observed in the spectrum due to the promotion of the core electron into one of the unoccupied orbitals of the molecule. Transitions to bound valence-shell and Rydberg orbitals will appear as peaks at photon energies below the ionization threshold, while transitions to quasi-bound shape resonances will be observed as broader peaks above threshold. Although the states at energies below threshold are neutral, they are nonetheless observed in photoionization spectra because the dominant decay mechanism in the soft $\mathrm{x}$-ray energy range is autoionization. In fact, ihe photoionization spectra are nearly equivalent to the corresponding $\mathrm{x}$-ray photoabsorption spectra. For the results presented here, the distinction between these two types of measurements is not significant. The study of near-edge $\mathrm{x}$-ray photoabsorption is also commonly referred to as X-ray Absorption Near-Edge Structure (XANES). Here that term will generally be restricted to soft $\mathrm{x}$-ray absorption and will also be used to describe photoionization spectra.

XANES spectra (and thus near-edge photoionization spectra) provide information about the orbitals of the molecule which are unoccupied in the ground electronic state. From a fundamental viewpoint, knowledge of these orbitals is needed even for an understanding of the ground state. Although within the Hartree-Fock approximation these orbitals are unoccupied, in fact they do influence ground-state properties through 
electron correlation, i.e. configuration interaction. These orbitals also play a role in the dynamics of a molecule, e.g. in a collision or chemical reaction. And these orbitals may be populated in the many different excited electronic states of the neutral or ionized molecule. Naturally the details of a given orbital may vary in these different circumstances, but XANES can provide at least a basic characterization of the unoccupied molecular orbitals.

Relative energies of the various unoccupied orbitals may be determined from XANES spectra. For example, molecular-field splittings of low-lying Rydberg orbitals can be directly measured. The spatial distribution of an orbital accessed by a XANES transition may be inferred from transition intensities, natural linewidths, and vibrational structure. Similar information can be obtained from lower-energy photoabsorption experiments, where a valence electron is excited into an unoccupied orbital. This approach is also useful, and may achieve better energy resolution than is possible with XANES. However core-level photoabsorption has several inherent advantages over valence photoabsorption. First of all, occupied valence levels have small energy separations. Therefore excitations from different valence levels will tend to form overlapping series of peaks, and it may be non-trivial to associate each observed transition with the correct valence hole. In contrast, core levels are widely separated in energy, and $\mathrm{x}$-ray absorption features are thus more easily assigned to the correct hole. Secondly, the symmetries of the various occupied valence orbitals are not always known, and it may therefore be difficult to identify the symmetry of orbitals accessed in transitions from these initial states. Core-level symmetries are more easily determined because these levels are very similar to their parent atomic orbitals. Thirdly, creation of a valence hole may have a dramatic effect upon the electronic and geometric structure of a molecule, because valence orbitals are often strongly bonding or antibonding. These hole-dependent changes may be difficult to distinguish from the influences of the newlyoccupied orbital. While a core hole will also have an influence upon the final-state 
structure, the hole is localized near one nucleus of the molecule, and will typically have a smaller influence than a valence hole. This influence is also more easily predicted. The effect of the core hole can often be modeled simply as the creation of a positive point charge at the nucleus.

Near-edge photoabsorption spectra also provide information about the core levels which are excited. For example, the natural line width of a transition is determined by the lifetime of the excited state. This lifetime is normally determined by the rate of decay of the core hole, by either autoionization or $\mathbf{x}$-ray fluorescence. High-resolution photoabsorption spectra may therefore be used to study trends in core-hole decay rates. These spectra are also useful for the measurement of various energy splittings in core levels. In particular, spin-orbit and molecular-field splittings may be determined and used to understand the effects of chemical bonding on atomic core levels.

Promotion of a core electron into a previously unoccupied orbital creates a very highly excited electronic state of the molecule. Structural parameters of that electronic state may be determined if vibrational transitions are resolved in the photoabsorption spectrum. Upon electronic excitation, it is possible to excite one or more quanta of vibrational energy in the molecule, resulting in a series of peaks associated with a single electronic transition. The vibrational energy spacings observed are a measure of the force constants of the molecule in the excited state. The relative intensities of the individual vibrational excitations can be analyzed using the Franck-Condon principle to derive bond lengths and bond angles of the molecule in the excited state.

High-resolution XANES spectra can provide some information about the structure and dynamics of highly excited electronic states. However, more complex measurements are possible which give more detailed characterizations of these states or which use these states to study other molecular properties. For example, measurement of the relative intensities of fragment ions can be used to characterize the decay dynamics of the states which are excited in XANES. Photoelectron and Auger electron spectroscopy can be 
used to determine the role of the excited electron in autoionization of the excited state. Resonance-excited $\mathbf{x}$-ray emission is a relatively new technique for studying occupied valence levels of the ground state. In these spectroscopies, and many others, a high resolution photoabsorption spectrum is useful for the interpretation of experimental results. High-resolution XANES can therefore be regarded not only as a tool with the specific abilities listed above, but also as a starting point for more sophisticated measurements.

\section{I.B: Historical development of soft x-ray absorption spectroscopy}

Measurements of $x$-ray absorption spectra date back to the beginning of this century, when Bragg diffraction was discovered and $\mathbf{x}$-rays monochromators were first constructed [1]. There was considerable activity in this area over the following decades, including studies of condensed matter as well as gas-phase atoms and molecules [2]. There were similar advances, starting in the middle of the $19^{\text {th }}$ century, in the area of ultraviolet (UV) absorption. Developments in both UV and X-ray absorption spectroscopy, along with major advances in quantum theory, created a framework for the interpretation of electronic excitation spectra. The resulting understanding of electronic structure and spectra is thoroughly described in Ref. [3] for atoms and Ref. [4] for molecules.

Historically, absorption spectra in the soft $\mathrm{x}$-ray region $(\approx 30-1000 \mathrm{eV})$ were difficult to measure due to the lack of intense sources of continuum radiation. Some spectra were measured using quasi-continuum spark sources [5], but the field was very limited until the 1960's, when synchrotron radiation first became available. It was immediately obvious that this was a superior source of soft $x$-rays, because of the nearly featureless continuum as well as the high flux. As better soft $\mathrm{x}$-ray monochromators were developed to exploit this radiation source, many near-edge molecular photoabsorption 
spectra were collected and the resulting interpretations and associated theoretical work established a basis for modern work [6]. By modern standards, however, most soft x-ray spectra collected before the mid-1970's were not truly high resolution.

In the 1970's the technique of Electron Energy Loss Spectroscopy (EELS) was improved, resulting in a truly high-resolution spectroscopy. EELS, which was developed in parallel with $x$-ray absorption, provides an alternate means of measuring absorption spectra. In EELS, a high-energy electron replaces the photon as the source of excitation energy. Since a free electron can occupy a continuum of energy levels, the incident electron can transfer any fraction of its energy to the molecule. Thus a spectrum is obtained by measuring the energy loss of the inelastically-scattered electrons, using a very narrow incident-beam energy distribution. The groups of King, at Cambridge University [7], and Brion, at University of British Columbia [8], developed gas-phase EELS instruments which could measure near-edge spectra with resolutions close to the natural linewidths of the spectral features. These instruments were used to study many atoms and molecules. The UBC group, in particular, studied an impressive number of molecules and systematically interpreted the results. The detailed understanding of nearedge spectra obtained from the work of the EELS groups is, in fact, the framework with which the present measurements were interpreted. However, for all its advantages and success, EELS has an inherently low count rate, and spectra usually have relatively small signal-to-noise ratios even for counting times of more than one day.

From the mid-1970's to the mid-1980's, EELS was the method of choice for measuring high resolution XANES in the soft $x$-ray energy range. The performances of soft $x$-ray monochromators were improving, however, and more synchrotron radiation facilities were being developed. The modern era of high-resolution XANES began in the late 1980 's, when several soft $x$-ray monochromators were commissioned with resolving powers (E/ $\Delta 5$ ) of 5000 or more. The first and still the most famous spectrum of the current period of activity is the molecular nitrogen $1 \mathrm{~s}-\mathrm{to}-\pi^{*}$ resonance, with well-resolved 
vibrational structure, collected by Chen and coworkers using the Dragon monochromator at the National Synchrotron Light Source (NSLS) [9]. Another Spherical Grating Monochromator (SGM) on Beam Line 6 at the Stanford Synchrotron Radiation Laboratory (SSRL) soon afterwards demonstrated a similar resolving power [10]. The next addition was the SX700/II monochromator [11] at the Berliner Elektronenspeicherring für Synchrotronstrahlung (BESSY), which provided the highresolution soft $x$-rays used for the measurements in this dissertation. Since the initial measurements at SX700/II, other high-resolution monochromators have heen commissioned at various synchrotron radiation facilities around the world.

As a result of the unprecedented combinations of resolution and flux provided by these new monochromators, there has been a recent flurry of activity in the area of highresolution XANES. Besides the work contained in this dissertation, and other work of the same group [12], the group using the Dragon monochromator, as well as a newer SGM at NSLS, has actively investigated a number of molecules [13]. Other groups are also working in this area, and even more may be expected as high-resolution, high-flux monochromators become widely available.

\section{I.C. Survey of present measurements}

This dissertation presents molecular soft $x$-ray photoionization spectra which, for the purposes of this study, are equivalent to the corresponding XANES spectra. The SX700/II monochromator at BESSY provided the combination of high flux and high resolution required. Absorption of $\mathrm{x}$-rays by the gas was detected by the total ion yield technique, in which a biased electrode is used to collect all ions produced by photoexcitation. Full details of the experiment are given in Chapter II.

XANES spectra of four different molecules will be presented and discussed. The first molecule studied in this series of experiments was carbon monoxide. The 
interpretation of the observed carbon and oxygen K-edge spectra is fairly straightforward, and introduces some concepts used in the interpretation of more complex spectra. In particular, the equivalent-core model is applied and an analysis based on Franck-Condon (FC) theory is demonstrated. Formaldehyde, because of its larger number of atoms, exhibits more complex carbon and oxygen K-edge XANES spectra than those of CO. Both $\mathrm{H}_{2} \mathrm{CO}$ and $\mathrm{D}_{2} \mathrm{CO}$ were studied, exploiting the isotopic dependence of vibrational frequencies for the assignment of vibrational features in the spectra. By applying a polydimensional FC analysis, the geometries of several excited states of both isotopomers were derived. An unprecedented isotopic effect was observed in the energy and width of the lowest-energy Rydberg state at both edges.

The XANES spectra of sulfur hexafluoride were measured at the sulfur $L_{2,3}$ edges and the fluorine $\mathrm{K}$ edge. The high symmetry and small size of the molecule allows a relatively simple interpretation of the observed spectra, even though this is a seven-atom molecule. The most striking result was the observation of many Rydberg states below the sulfur $L_{2,3}$ edges. The natural linewidths of these states are very small, especially in comparison to the linewidths of the intense inner-well resonance at slightly lower photon energy. XANES spectra were also measured at the sulfur $L_{1,2,3}$ edges of hydrogen sulfide. Considerable fine structure was resolved below the $L_{2,3}$ edges. Interpretation of the spectra was facilitated by measuring both $\mathrm{H}_{2} \mathrm{~S}$ and $\mathrm{D}_{2} \mathrm{~S}$. A detailed analysis revealed a molecular-field splitting of the sulfur $2 p$ core levels and suggested that some observed states had characteristics intermediate between the common valence-shell and Rydberg classifications. Vibrational structure and isotopic shifts were resolved in the valenceshell region and were interpreted by considering the recently-established dissociative nature of these excited states.

\section{I.D. Basic principle and common themes}


This dissertation is organized with separate chapters to discuss the results from each of the four molecules studied. This is a useful structure, but it may hide some of the common threads running through these different systems. In this section, the basic prinsiples underlying this research are introduced and connections between the different molecules are outlined.

\section{Rydberg states}

A Rydberg state arises from the promotion of an electron into a Rydberg orbital. Rydberg states are most easily defined as states whose energies are predicted by the Rydberg formula

$$
E_{\mathrm{n} \ell}=E_{\mathbb{I P}} \cdot R /\left(n-\delta_{\ell}\right)^{2},
$$

where $\mathrm{E}_{\mathrm{IP}}$ is the ionization potential of the electron's initial-state level, $\mathrm{R}$ is the Rydberg constant, $\mathrm{n}$ and $\mathrm{l}$ are the principal and orbital-angular-momentum quantum numbers of the Rydberg orbital, and $\delta_{\ell}$ is the quantum defect. The quantum defect depends on $\ell$ because of the differing degrees of core penetration for $s, p, d$ etc. orbitals. Generally $\delta_{\ell}$ will te largest for $s$ orbitals, which have no centrifugal barrier, and will decrease with increasing $\ell$. $\delta$ will also increase with $Z$, the nuclear charge, because the screening of the nuclear charge by the inner electrons becomes less efficient in larger atoms. Rydberg states will iypically be observed in one or more series with energies converging on $E_{I P}$ as predicted by Eqn. (1.1). Such series are identified for all the molecules discussed in this dissertation.

A useful quantity for the description of Rydberg states is the term value

$$
T=E_{I P} \cdot E_{n \ell} .
$$


Ignoring configuration interaction and relaxation effects, which are normally small, the term value is associated only with the energy of the electron in the Rydberg orbital. Thus the term value is especially useful for comparisons of Rydberg states corresponding to different absorption edges. While this dissertation is concemed only with core-excited electronic states, it is important to stress that Rydberg states also may arise when valence electrons are promoted to Rydberg orbitals. Indeed, these lower-energy Rydberg states are more thoroughly studied, and provide the basis for the modern understanding of Rydberg orbitals $[14,15,16]$.

The Rydberg formula successfully predicts the term values of Rydberg states in multi-electron atoms, if $\mathrm{n}$ is larger than the maximum $\mathrm{n}$ of the occupied orbitals in the ground state. Moreover, it is also successful for predicting term values of many excited states in molecules. The reason for this success is that Rydberg orbitals have a large spatial extent. As $\mathbf{n}$ increases, Rydberg orbitals have less amplitude within the molecule, and more amplitude far away from the center, where the potential is atomic-like. Since the energy of an orbital depends on the entire one-electron wavefunction, higher Rydberg orbitals will have atomic-like energies. Another consequence of the large spatial extent of these orbitals is that transitions to higher Rydberg states will be successively weaker, simply tue to diminishing Rydberg orbital amplitude in the region of the core electron. However dipole selection rules will not necessarily approach the atomic rules for increasing $n$, because the region of the Rydberg orbital which overlaps the core electron is within the molecule and feels the full molecular field. An example of this is seen in Chapter VI, where the $2 \mathrm{p}$ to np transitions in $\mathrm{H}_{2} \mathrm{~S}$ show appreciable intensity, although the transition is forbidden by atomic dipole selection rules.

Rydberg orbitals have little amplitude within the molecu' essentially non-bonding, especially for large values of $n$. However the excitation of a Rydberg state may nonetheless have accompanying changes in molecular geometry, 
because the influence of the newly-created hole must also be considered. Valence holes can clearly have a dramatic influence on molecular geometry, since valence orbitals may be strongly bonding or antibonding. Core holes will have a smaller but often notable influence upon molecular structure (as seen, for example, in Chapter III for CO). Therefore core-excited Rydherg states may in some cases show vibrational sidebands as a result of the geometry changes associated with the excitation.

\section{Valence-shell states}

A valence-shell state arises from the promotion of an electron into an unoccupied molecular orbital. The analogy in an open-shell atom would be the promotion of an electron into the partially occupied shell, i.e. the valence shell. Dipole-allowed transitions to valence-shell states tend to be more intense than corresponding Rydbergstate transitions, because molerular orbitals are spatially localized within the molecule and will have appreciable amplitude in the region of the ground-state electrons. Like Rydberg states, valence-shell states have been more thoroughly studied for valence excitations than for core excitations [14,15]. However, core-excitation is, in some ways, a more powerful means for studying molecular orbitals which are unoccupied in the ground state. For example, an unoccupied molecular orbital could be excited from a (highly localized) core level of each atom of a molecule, and the resulting relative intensities would provide a map of the orbital amplitude at the different atoms.

From simple molecular orbital theory, it is evident that molecular orbitals which are unoccupied in the ground state are usually anti-bonding. Putting an electron into an anti-bonding orbital will tend to lengthen the associated molecular bonds, inducing vibrational excitation in the final state. Therefore core-excited valence-shell states generally show more extensive vibrational sidebands than the corresponding Rydherg states. There is an important exception however: It is possible that the creation of the 
core-hole will have some compensating effect on the change in geometry, leading to a final state with a geometry closer to the ground state than might otherwise be expected. This is seen in Chapter III, for example, where more extensive vibrational structure is observed for the (C 1s) $)^{-1}$ Rydberg states than for the (C $\left.1 \mathrm{~s}\right)^{-1} \pi^{*}$ valence-shell state.

Core-excited valence-shell states are of particular interest because they may be used to determine the orientation of molecules adsorbed on surfaces. This technique, which is called NEXAFS [17], exploits the molecular symmetry of antibonding orbitals and the (usually) linear polarization of synchrotron radiation. The dependence of transition intensity upon the angle between the photon polarization vector and the surface normal reveals the orientation of the bond associated with the antibonding orbital. Some of the valence-shell states used in this approach lie above the ionization threshold of the excited core electron. In that case, the orbital accessed is only quasi-bound, i.e. the electron rapidly tunnels out of the orbital into the continuum of free-electron states and leaves the molecule. This special, but not unusual, type of valence-shell state is called a shape resonance. Chapter $\mathrm{V}$ includes a discussion of the shape resonances of $\mathrm{SF}_{6}$.

\section{Intermediate or mixed states}

The sharp division between valence-shell and Rydberg states which is perhaps suggested by the preceding discussion is not always valid. In fact, it is sometimes more accurate to classify a state as mixed, i.e. intermediate between these two extremes. Chapter VI presents evidence of a mixed state in the core-excitation spectrum of formaldehyde. Another example is seen in Chapter VI for $\mathrm{H}_{2} \mathrm{~S}$. In that case, the spectral region between clearly identifiable valence-shell and Rydberg states shows some states with intermediate characteristics. The nature of mixed states has been more thoroughly investigated for valence excitations. While there is a general agreement that mixing is important in some situations, there is some controversy over the manifestation of that 
mixing. Robin [14] has argued that mixing occurs between conjugate pairs of valenceshell and Rydberg orbitals, generally resulting in a pair of mixed orbitals, one more Rydberg-like and the other more valence-shell-like. He stresses that transitions to both orbitals should be observed, although sometimes the transition to the valence-shell-like orbital will be very broad and difficult to identify. In contrast, Mulliken [18] has argued that mixing does not necessarily result in two distinct orbitals. The analysis used in this dissertation tends to follow Mulliken, i.e. identifying individual mixed states rather than conjugate pairs, but the results do not depend on the specific assumption of either point of view.

\section{Vibrational structure}

The promotion of an electron into a previously unoccupied orbital will generally change the geometry of a molecule, i.e. modifying the bond lengths and bond angles. Changes in geometric structure tend to induce the excitation of vibrational quanta in the final state. These excitations are observed as peaks at higher energies than the fundamental electronic transition, with spacings characteristic of the molecular vibrational frequencies in the excited state. This explains the sidebands observed for many transitions in molecular XANES spectra. Understanding the vibrational structure associated with a particular excited electronic state can provide details of the influence of the hole and the spatial distribution of the excited electron. Vibrational structure is observed and interpreted for all the molecules studied in this dissertation.

In complicated XANES spectra it may be difficult to assign all the observed features. It is very helpful to have additional information. Such information is available in the spectra of isotopically-substituted molecules. Different isotopic species of a molecule tend to have very similar electronic and geometric structures. However, due to the changes in atomic mass, they tend to have different vibrational frequencies. These 
differences are naturally very large when deuterium is substituted for hydrogen, because the mass changes by a factor of two. The vibrational frequencies in the excited states will be modified in the substituted molecule, and any vibrational sidebands in the spectra will show corresponding shifts. Thus this effect is useful for distinguishing vibrational sidebands from fundamental electronic transitions in a spectrum. The effect will, of course, be largest for those normal vibrational modes which include significant motion of the hydrogen/deuterium atoms. The energies of fundamental transitions, i.e. those without any vibration excitation, may also show small shifts upon isotopic substitution due to zero-point energy changes, as discussed below in Section I.D.6. Isotopic substitution is used in Chapters IV $\left(\mathrm{H}_{2} \mathrm{CO}\right.$ vs. $\left.\mathrm{D}_{2} \mathrm{CO}\right)$ and $\mathrm{VI}\left(\mathrm{H}_{2} \mathrm{~S}\right.$ vs. $\left.\mathrm{D}_{2} \mathrm{~S}\right)$ to aid in the interpretation of complicated spectra.

Once vibrational sidebands are identified in a XANES spectrum, it is immediately possible to determine vibrational frequencies of the excited electronic state from the spacing of the sidebands. This provides some information about the force constants, and thus the electronic structure of the excited electronic state, especially by comparison to ground-state vibrational frequencies. In polyatomic molecules, the vibrational spacings may also indicate changes in molecular symmetry, although this effect is not observed for the examples in this dissertation. Further information about the structure of the excited state is contained in the intensities of the vibrational sidebands. By applying the FranckCondon (FC) principle[19], bond lengths and angles in the excited state may be determined. The most important prediction of the FC principle is that extensive vibrational excitations tend to accompany large changes in molecular geometry. As mentioned above, in Sections I.D.1 and I.D.2, changes in molecular geometry are indicative of the spatial distribution of the excited valence-shell or Rydberg orbital, as well as of the influence of the core hole. Therefore FC analysis is a particularly useful tool for the interpretation of molecular XANES spectra. 


\section{Equivalent-core approximation}

As mentioned above, the creation of a core hole may have a notable influence upon the electronic and geometric structure of the excited electronic state. However this influence can usually be modeled by very simply by approximating the core hole as an additional positive charge at the nucleus of the atom. In other words, one can assume that the core orbital is localized in an extremely small region and thus that the core hole is a point charge. In this case, the core-excited atom is equivalent to a ground-state atom with one additional proton, i.e. the next element on the periodic table. Thus this model is known as the $\mathrm{Z}+1$ or equivalent-core approximation. Note that this model is also useful in the case where the excited electron has left the system, i.e. the final state in photoelectron spectroscopy. Indeed, the model was initially developed to describe the core-excited ions created in photoelectron spectroscopy [20]. To apply the equivalentcore model in XANES, it is important to consider the excited electron. If the core electron is promoted into the lowest unoccupied molecular orbital (LUMO), then the equivalent core molecule is the neutral $\mathrm{Z}+1$ molecule in the ground state, because the "extra" electron of the $\mathrm{Z}+1$ molecule occupies this LUMO. But if the transition is to a Rydberg state, then the equivalent-core molecule is the neutral $\mathrm{Z}+1$ molecule in an excited electronic state, with its outermost electron promoted into the same Rydberg orbital.

This model is applied in Chapter III for $\mathrm{CO}$ and the results show that the approximation is quite successful. If vibrational frequencies are corrected for the wellunderstood effects of different masses, the overall agreement between the core-excited values and the equivalent-core values is good. Bond lengths for core-excited states, derived using a FC analysis, also compare well to corresponding equivalent-core values. The success of the core model in the test case of $\mathrm{CO}$ is encouraging, and therefore the equivalent core model is also applied to the other three molecules studied in this 
dissertation. This model has two important applications. It can be used to predict the influence of the core hole in a core-excited electronic state. It can also be turned around, using XANES as a way to study the structure of the equivalent-core molecule [21]. The latter application is natural, in a way, because the first valence-shell state of a coreexcited closed-shell molecule, corresponds to an open-shell equivalent-core molecule in its ground state. Since closed-shell molecules are usually much more stable and inert than open-shell molecules, this approach is a simple way to learn about molecules which may be difficult to study directly. The results for $\mathrm{H}_{2} \mathrm{CO}$ in Chapter IV and $\mathrm{H}_{2} \mathrm{~S}$ in Chapter VI are both discussed in this light.

\section{Dissociative states and zero-point vibrational energy}

The core-excited electronic states accessed in XANES have very short lifetimes, due to the rapid autoionization processes associated with core holes created in the soft $\mathrm{x}$ ray energy range. However it is possible for a core-excited state to dissociate before this electronic decay occurs, i. e. the molecule fragments with a core hole in one of the pieces. Very rapid dissociation is most likely to occur if the excited state accessed has a strongly repulsive potential. Another possibility is that the excited state is bound, but is so strongly coupled to a dissociative state that conversion to the repulsive potential and subsequent dissociation occurs before the core hole decays.

One effect of dissociation is to shorten the lifetime of the excited state, as compared to a corresponding non-dissociative state which decays by autoionization. Therefore a dissociative state may be characterized a larger natural line width than nondissociative states in the same spectrum. There are other possible causes for differences in line broadening, however. The rate of autoionization may vary for different states in the same spectrum, as proposed in Chapter $\mathrm{V}$ for $\mathrm{SF}_{6}$. 
Another uffect of dissociation can be observed by comparing the corresponding XANES spectra of different isotopic species (or "isotopomers") of the molecule. Dissociative states will exhibit shifts in the energy of the fundamental peak upon isotopic substitution (for this effect, the final state of the transition must have a repulsive potential, rather than rapidly coupling to another state which is dissociative). These shifts arise from changes in the zero-point vibrational energy of the molecule upon excitation. Even in the vibrational ground state of a particular electronic state, each vibrational mode contributes to the total energy of a molecule [i.e. the energy of the vibronic (vibrationalelectronic) state]. Assuming a harmonic oscillator potential, this zero-point energy is given by

$$
\mathrm{E}_{\mathrm{zp}}=\sum_{i} \frac{\hbar \omega_{i}}{2}
$$

where the sum is over all the vibrational modes, and $\hbar \omega_{i}$ is the vibrational spacing of the $i^{\text {th }}$ mode. Normally these zero-point vibrational energies are difficult to distinguish from the total electronic energy of the overall molecular state. However, a comparison of total energies between isotopomers would show these effects, due to the isotopic dependence of vibrational frequencies. Of course, total energies are not observed in absorption experiments; only the diffcrence in total energy between the initial and final state is measured. If vibrational frequencies are similar in these two states, there will be little change in the zero-point energy and thus only a small difference between the fundamental transition energies of the isotopomers. If the final state is dissociative, however, there is no potential well along the dissociating coordinate, and therefore no zero-point energy for that mode. The dissociative mode contributes to the zero-point energy in the initial state but not in the final state. Since that contribution is just half the vibrational spacing for that mode, there will be a shift of the fundamental transition upon isotopic substitution. This shift will equal half the difference between the ground state vibrational spacing in 
the two isotopomers. For the case of deuteration, the fundamental transition will shift to higher energies for the deuterated species.

Even a vibrational mode which is not dissociative may cause isotopic shifts in the fundamental transition energy, if the frequency of that mode changes upon excitation. However, the best-resolved spectra in this dissertation can only detect energy shifts of more than $\approx 10 \mathrm{meV}$. To obtain this shift upon deuteration, a change in the vibrational spacing of about $65 \mathrm{meV}$ for a mode involving motion of the hydrogen/deuterium would be required upon excitation. This corresponds to a change of more than $20 \%$ in the vibrational frequency for a stretching mode, and a much larger relative change for a bending mode. Of course, there may be several modes which change frequency and contribute to the isotopic shift. The conclusion is that only large changes in vibrational frequency can lead to observable shifts, and only modes with large isotopic dependencics, i.e. those that involve motion of the hydrogen/deuterium, will make significant contributions to the isotopic shifts. Clearly the largest possible change in a vibrational frequency occurs when the final state frequency is zero, i.e. the mode is dissociative. However it must be stressed that this effect may also be observed for completely nondissociative states if there is a large enough change in vibrational frequencies between the ground and final states. In Chapter IV there is a discussion of the isotopic shifts of fundamental transitions in reference to $\mathrm{H}_{2} \mathrm{CO}$ and related molecules. In Chapter VI this effect is used to explain isotopic shifts in transitions to states which are known, from other studies, to be dissociative.

\section{Core-hole spin-orbit interaction}

For core levels with orbital angular momentum greater than zero, i.e. for $\mathrm{p}, \mathrm{d}$, etc. levels, there is an observable splitting in XANES spectra due to the spin-orbit interaction of the core hole. This splitting, and the associated branching ratio, will sometimes also 
include contributions from the interaction of the core hole with the excited electron. The observable effects of the latter interaction provide information on the spatial distribution of the excited electron. This effect is discussed in Chapters $\mathrm{V}$ and $\mathrm{VI}$ in reference to $\mathrm{SF}_{6}$ and $\mathrm{H}_{2} \mathrm{~S}$, respectively. In the latter case, there is also a modification of the observed spinorbit splitting in XANES due to molecular-field effects on the core levels. More detailed introductions to these phenomena are given as needed in Chapters V and VI.

\section{E. Organization of chapters}

Chapter II presents the technical details of the experiments. The following chapters present XANES spectra along with the corresponding analysis and discussion. Chapters III, IV, V, and VI discuss $\mathrm{CO}, \mathrm{H}_{2} \mathrm{CO}, \mathrm{SF}_{6}$, and $\mathrm{H}_{2} \mathrm{~S}$, respectively. Chapter VII presents some ideas for future work in this area. 


\section{REFERENCES:}

1. A. Compton and S. Allison, X-Rays in Theory and Experiment (Van Nostrand, New York, 1936).

2. L. G. Parratt, Rev. Mod. Phys. 31, 616 (1959).

3. R. D. Cowan, The Theory of Atomic Structure and Spectra (Univ. of California Press, Berkeley, 1981).

4. G. Herzberg, Molecular Spectra and Molecular Structure: Vol. I: Spectra of Diatomic Molecules (Van Nostrand, Princeton, 1950) Second Edition, Vol. III: Electronic Spectra of Polyatomic Molecules (Van Nostrand, New York, 1966).

5. D. H. Tomboulian, Handbuch der Physik (Springer-Verlag, Berlin, 1957) Vol. 30, p. 246.

6. F. Brown, Ch. 4, and G. S. Brown and S. Doniach, Ch. 10 in Synchrotron Radiation Research, ed. H. Winick and S. Doniach (Plenum, New York, 1980).

7. G. C. King and F. H. Read, in Atomic Inner-Shell Physics, ed. B. Crasemann (Plenum, New York, 1985), pp. 317-375; M. Tronc, G. C. King, and F. H. Read, J. Phys. B 12, 137 (1979).

8. See, for example, A. P. Hitchcock and C. E. Brion, J. Electr. Spectrosc. 10, 317 (1977); ibid, 13, 193 (1978); ibid, 14, 417 (1978); ibid, 15, 401 (1979); ibid, 18, 1 (1980); ibid, 19, 231 (1980).

9. C. T. Chen, Y. Ma, and F. Sette, Phys. Rev. A40, 6737 (1989).

10. P. A. Heimann, F. Senf, W. McKinney, M. Howells, R. D. van Zee, L. J. Medhurst, T. Lauritzen, J. Chin, J. Meneghetti, W. Gath, H. Hogrefe, and D. A. Shirley, Phys. Scr. T31, 127 (1990).

11. M. Domke, T. Mandel, A. Puschmann, C. Xue, D. A. Shirley, G. Kaindl, H. Petersen, and P. Kuske, Rev. Sci. Instrum. 63, 80 (1992).

12. M. Domke, C. Xue, A. Puschmann, T. Mandel, E. Hudson, D. A. Shirley, G. Kaindl, C. H. Greene, H. R. Sadeghpour, and H. Petersen, Phys. Rev. Lett. 66, 1306 (1991); M. Domke, G. Remmers, and G. Kaindl, Phys. Rev. Lett. 69, 1171 (1992); G. Remmers, M. Domke, and G. Kaindl, Phys. Rev. A 47, 3085 (1993); G. Remmers, M. Domke, A. Puschmann, T. Mandel, G. Kaindl, E. Hudson, and D. A. Shirley, Chem. Phys. Lett., submitted.

13. Y. Ma, C. T. Chen, G. Meigs, K. Randall, and F. Sette, Phys. Rev. A44, 1848 (1991); F. X. Gadea, H. Köppel, J. Schirmer, L. S. Cederbaum, K. J. Randall, A. M. Bradshaw, Y. Ma, F. Sette, C. T. Chen, Phys. Rev. Lett. 66, 883 (1991); J. Schirmer, A. B. Trofimov, K. J. Randall, J. Feldhaus, A. M. Bradshaw, Y. Ma, C. T. Chen, and F. Sette, Phys. Rev. A 47, 1136 (1993).

14. M. B. Robin, Higher Excited States of Polyatomic Molecules (Academic, New York, 1974), Vol. I. 
20

15. M. N. R. Ashford, M. T. Macpherson, and J. P. Simons, Top. Curt. Chem. 86, 2 (1979); C. Sandorfy, Top. Current Chem. 86, 91 (1979).

16. A. B. F. Duncan, Rydberg Series in Atoms and Molecules (Academic Press, New York, 1971).

17. J. Stour and R. Jaeger, Phys Rev, B 26, 4111 (1982); J. Stor, NEXAFS Spectroscopy (Springer-Verlag, Berlin, 1992).

18. R. Mulliken, Accounts of Chemical Research 9. 7 (1976).

19. E. Hutchisson, Phys. Rev. 36, 410 (1930); Phys. Rev. 37, 45 (1931); T. E. Sharp and H. M. Rosenstock, J. Chem. Phys. 41, 3453 (1964).

20. J. M. Hollander and W. L. Jolly, Acc. Chem. Res. 3, 193 (1970); W. L. Jolly, in Electron Spectroscopy: Theory, Techniques, and Applications, ed. C. R. Bridle and A. D. Baker (Academic Press, New York, 1978) p. 119.

21. W. H. E. Schwarz, Angew. Chem. Internat. Edit. 13, 454 (1974). 


\section{CHAPTER II: EXPERIMENT AND DATA ANALYSIS}

\section{II.A. Synchrotron radiation}

Synchrotron radiation was used as the radiation source for all the measurements presented in this dissertation. The primary advantage of synchrotron radiation in this case is that it provides a nearly structureless continuum, i.e. flux is a smooth function of photon energy. The inherently high flux in the soft $x$-ray energy range is another useful aspect of this radiation. Synchrotron radiation is produced when charged particles at relativistic energies experience a centripeta] acceleration [1]. Electron (or positron) storage rings have been constructed at many locations around the world to exploit this effect.

\section{II.B. The SX700/II monochromator}

To measure photoionization spectra, the continuum of photon energies provided hy the storage ring must be monochromatized. All the spectra presented here were measured using the SX700/II monochromator $[2,3]$, which is operated hy the Free University of Berlin at the storage ring BESSY. The combination of high photon flux and high resolution in energy provided by this monochromator is essential for the measurement of high-resolution XANES. Fig. II-1 shows a schematic of the monochromator optics, as well as a plot of calculated and measured energy resolution as a function of photon energy. The monochromator has only three optical elements and no entrance slit, using instead the electron beam in the storage ring as the monochromator entrance "aperture". These features lead to an inherently high flux. Only one optical element is non-planar, and that (ellipsoidal) mirror was made to a very high tolerance. Because the ellipsoidal mirror has smaller figure errors over shorter length scales, the optical aberrations can be reduced by shading most of the mirror and using only the central portion. This improves the energy 
resolution at the expense of flux. All of the spectra shown in this dissertation were measured with some shading of the ellipsoidal mirror; the exact amount of shading was selected for the optimum combination of flux and resolution in each case.

Another controllable contribution to the resolution is the verticul size of the electron beam in the ring. Because there is no entrance slit, this source size has a direct influence on the resolution. Some of the spectra discussed in Chapters III and V were obtained using special small-source beam conditions in the storage ring, allowing higher resolution measurements. The exit slit size also contributes to the final resolution, but this parameter is more easily adjusted. Several slit sizes are available, with smaller slits providing hetter

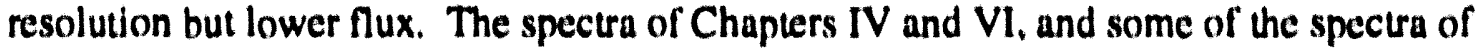
Chapter V, were obtained using a 2442 line/mm grating. This grating provides significantly better combinations of flux and resolution than the $1221 \mathrm{line} / \mathrm{mm}$ grating used for the earlier measurements. The resolution values plotted in Fig. II-1 refer to the 1221 line/mm grating. In the first order of diffraction, with a normal source size, the 2442 line/mm grating provides resolution comparable to line (b) in Fig. II-1. Further details of monochromator operation are provided in the individual discussions of experimental details in the following chapters.

In addition to the results presented in this thesis, the $S \times 7(0) / 11$ monochromator has heen used to study the high-resolution photoionization spectra of several atoms and molecules in the gas phase $[3,4]$. Its high resolution has also been exploited for a number

of other experiments. For example, adsorhed molecules were studied by NEXAFS [5] and by photon-stimulated desorption [6]. A complete description of research at the SX7(X)/II is provided in a recent report [7].

\section{II.C. Photoionization gas cell}

To obtain the hest possible high-resolution XANES spectra using the SX700/1I, a 
sensitive means of detecting photoabsomtion in the sample gas is required. This was achieved using a photoionization gas cell. As shown in Fig. Il-2, the cell contains the sample gas at pressures $<0.1$ mbar, separated from the ultra-high vacuum of the monochromator by a thin window $(-10(x)-15(x) \AA)$. Absorption of soft $x$-rays by the sample gas in the interaction region is detected by the total ion yield method, i.e. all the ions produced hy photoexcitation are collected and the ion current is measured. Specifically, the soft $x$-ray heam passes between two parallel copper plates in the gas cell for a distance of $10 \mathrm{~cm}$. A voltage of $+!(X) \mathrm{V}$ is applied to one plate, forcing positive photo-ions to the opposite plate, which is connected to a picoammeter. It is preferable to collect ions rather than electrons, hecause some photoelectrons may be ejected from the window or from wherever stray $x$-rays strike a surface within the gas cell.

Although the excited states studied in these experiments are neutral, the excited molecule or its dissociation fragments rapidly decay hy emitting one or more electrons. The resulting ion current provicies a measure of the photoabsorption cross section. To be exact, the total ion yield technique gives a photoabsorption spectrum which is weighted hy the average total positive charge produced by the molecule upon excitation at a given photon energy. It is important to recognize that many different linal states may result from these high-energy excitations, possibly including several highly-charged fragment ions from a single excited molecule. Thus the total positive charge produced may vary among the different final states, and the hranching ratio to these different final states may have some spectral dependence. For example, if one resonance in a spectrum produces significantly more or less total positive charge than another resonance, then the spectrum measured by total ion yield will show a different intensity ratio than the corresponding photoabsorption spectrum. Therefore relative intensities ohserved in total ion yield spectra should be carefully interpreted, as they may not exactly represent the absorption spectrum. In practice, these differences should not the very large. In this dissertation, the measured photoionization spectra are generally assumed to he equivalent to photoabsorption spectra. 
but the conclusions presented are expected to be valid even if this equivalence is not exact. Moreover, a general comparison of photoionization spectra to photoabsorption spectra demonstrates that this assumption is reliable in many different cases.

Spectra obtained using the total ion yield technique are subject to a systematic "saturation" error if the sample gas pressure is too high. This is caused by absorption of $x$ rays in the volume of gas between the window and the leading edges of the electrodes. lons produced in some portion of this volume will collected by the cathode, i.e. they are in the active region of the ionization cell, but generally some ions will not be detected. Therefore the photon flux reaching the active region will be attenuated by the gas in the "dead" volume, as predicted by the Beer-Lambert Law

$$
\frac{1}{10}=10^{-(000)}
$$

where Io is the incident photon flux, I is the transmitted flux, $\sigma$ is the photoabsorption cross section. $c$ is the concentration of absorber, and $b$ is the path length between the window and the active region. If the concentration $\mathrm{c}$ is large enough, changes in the cross section $\sigma$ as the photon energy is varied will cause measurable changes in the amount of flux reaching the active region. In that case, a peak in the total ion yield spectrum will appear broader and less intense than it otherwise would, hecause at the center of the peak the "dead" volume attenuates more photon flux than at the edges. This saturation effect may be avoided if the concentration, i.e. the gas pressure, is so low that the dead volume does not absorb appreciable flux, even at peak maxima in the spectrum. The saturation pressure may be determined empirically by comparing the shapes of the intense absorption features in spectra measured at different pressures. All the spectra presented in this dissertation were tested for saturation by comparison to spectra at higher pressures, and found to be unsaturated. Of course, saturation effects may be reduced by designing a gas cell with the smallest possible dead volume, i.e. minimizing $\&$ by placing the electrodes 
very close to the window. This would effectively increase the maximum measurement pressure, leading to higher signal-to-noise ratios.

\section{II.D. Data analysis}

To interpret the photoionization spectra in this dissertation, it was necessary to extract various parameters from the data. In the limit of perfect instrumental resolution, i.e. $\Delta E=0$, peaks in XANES spectra normally have a Lorentzian lineshape. Each individual transition is then characterized by an energy, a natural linewidth, and an intensity. The energy of the transition is the difference between the final (core-excited) and initial (ground) states. The Lorentzian (natural) linewidth corresponds to the lifetime broadening of the state. The intensity (area) is proportional to the cross-section for the transition. In practice, an instrumental contribution to the linewidth must also be considered, due to the limitations even of the highest-resolution experiments. For the SX700/1I monochromator, the resolution function may be roughly approximated by a Gaussian lineshape, although better approximations are possible [3]. Deviations from this approximation should not significantly affect the conclusions of the present work.

Because the natural linewidths tend to be large for core-excited states, XANES spectra often include regions of overlapping peaks. For example, vibrational spacings are often comparable to or less than natural linewidths and/or spectral resolution, and Rydberg states inherently have smaller energy separations as the excitation energy increases towards threshold. To best understand such spectra, it is desirable to deconvolute these regions of overlapping peaks, remove the instrumental linewidth, and thereby obtain the energy, natural linewidth, and relative intensity of each individual absorption peak. In the present

work, this deconvolution was achieved, as much as possible, hy analyzing the spectra with a least-squares-minimization curve-fitting routine.

Each individual peak was modeled by a Voigt function, i.e. by a Lorentzian 
function convoluted with a Gaussian function. One contribution to the Gaussian linewidth stems from instrumental broadening due to the finite resolution of the monochromator. Unresolved vibrational progressions associated with one electronic excitation will cause additional broadening, which can be approximated by a Gaussian lineshape. In general, the total Gaussian linewidth derived from a fit will reflect both of these contributions.

The data analysis presented in this dissertation often required considerable curvefitting to extract the significant features of the spectra. This least-squares analysis was accomplished using a VAX-based software package XFIT [8], which allows great flexibility in the specification of the fit functions. For example, the Franck-Condon (FC) fits of Chapters III, IV, and IV were accomplished by interfacing an existing FC code with XFIT. 


\section{REFERENCES:}

1. H. Winick, Ch. $1 \& 2$ in Synchrotron Radiation Research, ed. H. Winick and S. Doniach (Plenum, New York, 1980).

2. H. Petersen, Opt. Comm. 40, 402 (1982); Nucl. Instrum. Methods A246, 260 (1986).

3. M. Domke, T. Mandel, A. Puschmann, C. Xue, D. A. Shirley, G. Kaindl, H. Petersen, and P. Kuske, Rev. Sci. Instrum. 63, 80 (1992); G. Kaindl, M. Domke, C. Laubschat, E. Weschke, and C. Xue, Rev. Sci. Instrum. 63, 1234 (1992);

4. M. Domke, C. Xue, A. Puschmann, T. Mandel, E. Hudson, D. A. Shirley, G. Kaindl, C. H. Greene, H. R. Sadeghpour, and H. Petersen, Phys. Rev. Lett. 66, 1306 (1991); M. Domke, G. Remmers, and G. Kaindl, Phys. Rev. Lett. 69, 1171 (1992); G. Remmers, M. Domke, and G. Kaindl, Phys. Rev. A 47, 3085 (1993); G. Remmers, M. Domke, A. Puschmann, T. Mandel, G. Kaindl, E. Hudson, and D. A. Shirley, Chem. Phys. Lett., submitted.

5. See, for example: T. Solomun, K. Christmann, and H. Baumgärtel, J. Phys. Chem. 93, 7199 (1989); H. Rabus, D. Arvanitis, M. Domke, A. Puschmann, L. Wentz:1, G. Comelli, G. Kaindl, and K. Baberschke, Physica Scripta T31, 131 (1990); A. Puschmann, T. Mandel, M. Domke, C. Xue, and G. Kaindl, Surf. Sci. 252, 353 (1991); H. Rabus, D. Arvanitis, M. Domke, and K. Baberschke, J. Chem. Phys. 96, 1560 (1992); D. Arvanitis, J. Singh, H. Rabus, T. Lederer, and K. Baberschke, Phys. Rev. B 45, 1518 (1992).

6. P. Feulner, R. Scheuerer, M. Scheuer, G. Remmers, W. Wurth, and D. Menzel, Applied Phys. A55, 478 (1992).

7. SX700/II Beamline at BESSY Operated by Freie Universität Berlin Progress Report (1989-1992), ed. M. Domke and G. Kaindl (Freie Universität Berlin internal report, 1993).

8. XFIT was developed by C. Xue, in Frof. G. Kaindl's research group at the Free University of Berlin. 


\section{FIGURE CAPTIONS:}

Figure II-1: (Bottom) Schematic view of the SX700/II monochromator. (Top) The monochromator resolution $\triangle \mathrm{E}$ (FWHM) as a function of photon energy. The straight lines indicate the expected relation $\Delta E \alpha E^{3 / 2}$. (a) Normal source size, first order of diffraction. (b) Small source size, first order of diffraction. (c) Small source size, second order of diffraction.

Figure II-2: Schematic view of the ionization cell used for the measurement of gas-phase total-ion-yield spectra. 

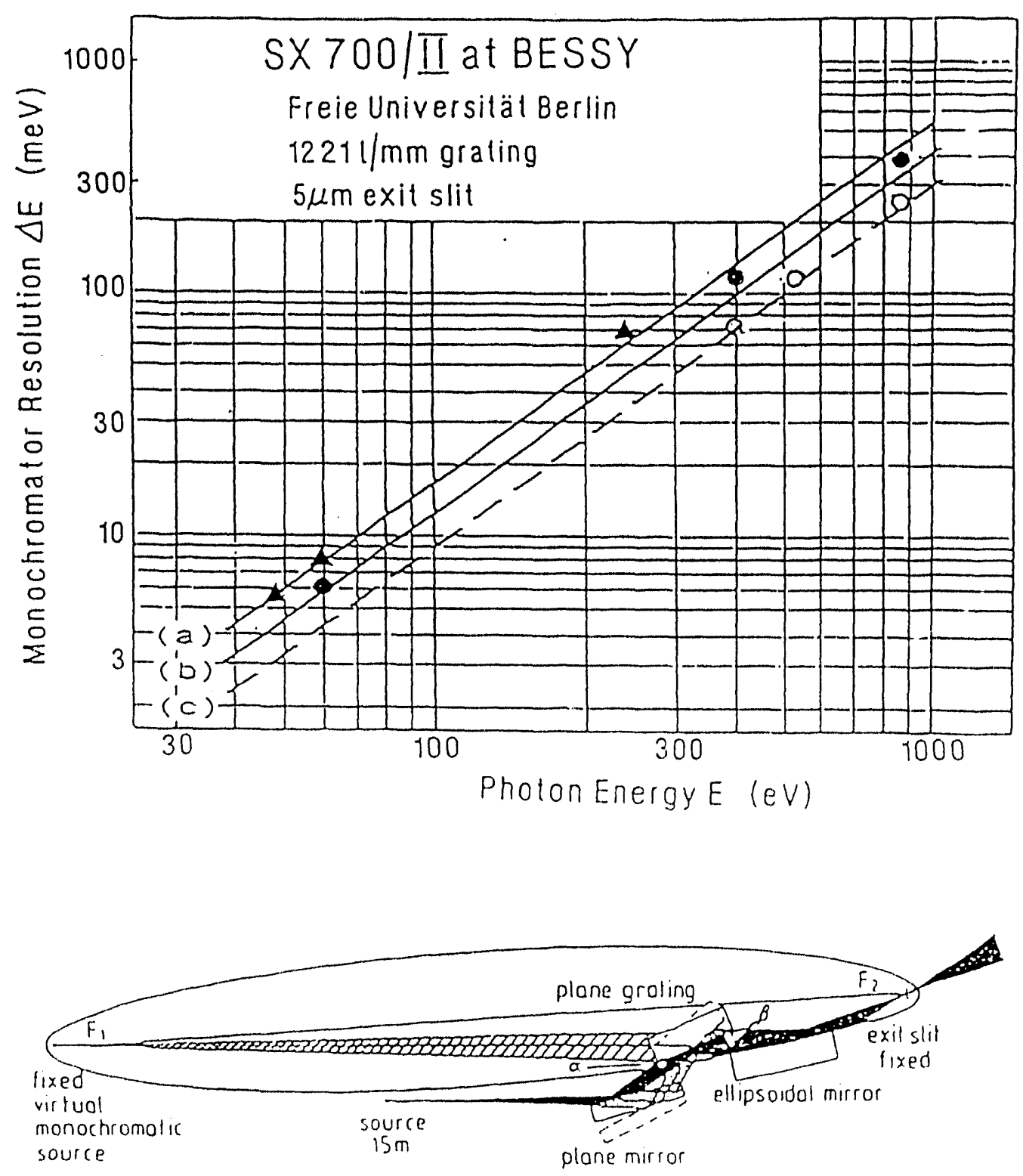

Figure II-1 


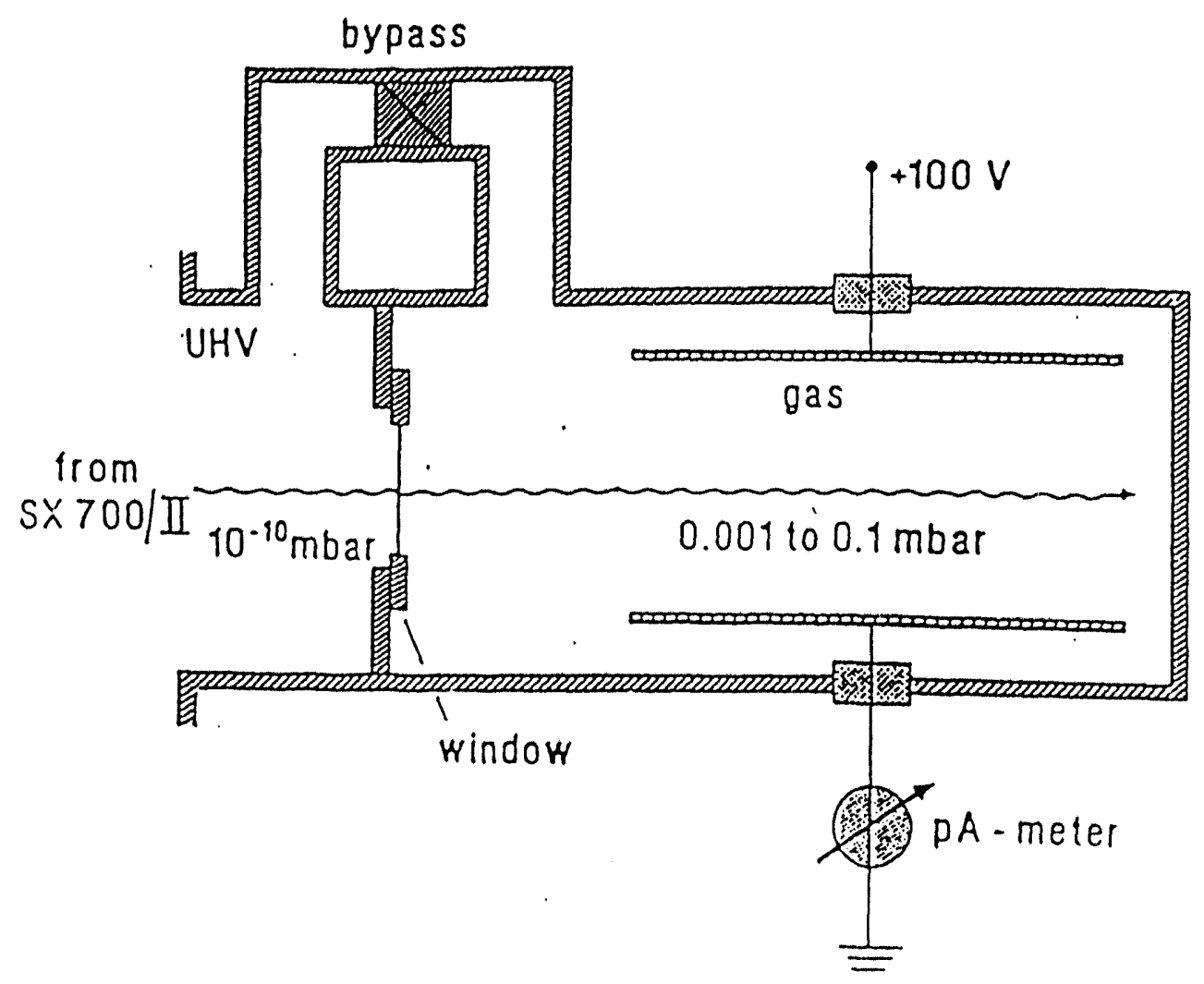

Figure II-2 


\section{CHAPTER III: HIGH-RESOLUTION PHOTOIONIZATION OF CARBON MONOXIDE AT THE CARBON AND OXYGEN K- EDGES}

\section{III.A. Introduction}

The carbon [1,2] and oxygen [3] K-edge XANES of carbon monoxide has been studied previously with high resolution in energy using Electron Energy Loss Spectroscopy (EELS). In the present experiment, using soft $x$-rays from the SX700/II monochromator, the spectral resolution was improved at the oxygen K-edge, and the signal-to-noise ratio was significantly improved at both edges. The resulting spectra show previously unobserved features, especially in the carbon K-edge Rydberg states and the oxygen K-edge valence-shell and Rydberg states. A Franck-Condon analysis is applied to the observed vibrational structure. The derived excited-state vibrational spacings and bond lengths are compared to the known values for the corresponding equivalent-core-molecule electronic states.

\section{III.B. Experimental}

The spectra were measured using the SX700/II monochromator with the 1221 line/mm grating. At the carbon K-edge, in the second order of diffraction, the spectral resolution was $70 \mathrm{meV}$ (FWHM). At the oxygen K-edge, with small-source beam conditions and second-order diffraction, the resolution was $\approx 115 \mathrm{meV}$. High-purity carbon monoxide (99.997\%) at pressures of $\approx 0.05$ mbar was used, with a $1500 \AA \mathrm{Al}(1 \%$ $\mathrm{Si})$ window separating the monochromator from the gas cell. 


\section{III.C. Carbon K-edge}

\section{Overview}

Fig. III-1 shows the photoionization spectrum of $\mathrm{CO}$ near the carbon K-edge. The lowest-energy feature is the (C $1 s)^{-1} \pi^{*}$ resonance, plotted with an expanded energy scale in the inset of Fig. III-1. A series of less intense features, converging on the carbon $1 \mathrm{~s}$ ionization threshold at $296.1 \mathrm{eV}$, are assigned to transitions to Rydberg states with vibrational sidebands. Assignments and derived energies of the individual states are given in Table III-I. Above threshold there is further structure, assigned to two-electron excitations. These $2 \mathrm{~h}$-2e states arise from the simultaneous promotion of the $\mathrm{C} 1 \mathrm{~s}$ electron and a valence electron into the $\pi^{*}$ and Rydberg orbitals.

\section{2. (C $1 \mathrm{~s})^{-1} \pi^{*}$ resonance}

Four vibrational transitions, from $0 \rightarrow 0$ to $0 \rightarrow 3$, were resolved for the (C $1 \mathrm{~s})^{-1} \pi^{*}$ icsonance, as indicated in the inset of Fig. III-1. A Franck-Condon analysis, with groundstate parameters $h v^{\prime \prime}=269.0 \mathrm{meV}$ and $\mathrm{R}^{\prime \prime}=1.1283 \AA$ [4], resulted in the excited-state parameters $h v^{\prime}=256(2) \mathrm{meV}, h v x^{\prime}=3.6 \mathrm{meV}$ and $R^{\prime}=1.153(1) \AA$, where the uncertainty ir indicated in parenthesis in units of the last digit. (Double primes indicate ground state parameters while primes refer the to excited state.) The resulting fit is plotted with the data in the inset of Fig. III-1. These results agree fairly well with those of Refs. [1,2], with smaller errors. Note that in Ref. [2] it was suggested that the bond length decreases relative to the ground state. The present results definitively show that the bond length increases, i.e. the excitation is to the repulsive side of the potential. 


\section{3. (C 1s) $)^{-1}$ Rydberg resonances}

Fig. III-2 shows the fine structure observed below the $\mathrm{C}$ 1s ionization threshold. These features are assigned, as indicated in Fig. III-2 and Table III-I, to excitation of the C is electron into various Rydberg orbitals, with accompanying vibrational sidebands. This interpretation agrees with earlier assignments, with some small differences and with more states resolved. Note that core-excited carbon corresponds to ground-state nitrogen, within the $\mathrm{Z}+1$ approximation. Thus the present assignments were guided by well-established assignments for the cquivalent-core states, i.e. the excited states of NO arising from the promotion of the (valence) electron in the $2 \pi$ molecular orbital (the $\pi^{*}$ orbital of $\mathrm{CO}$ ) into the Rydberg orbitals. A comparison of term values is shown in Table III-1and discussed again in Section III.E.

The two prominent triplets in the energy range $292-294 \mathrm{eV}$ are assigned to the (C $1 \mathrm{~s})^{-1} 3 \mathrm{~s} \sigma$ and $(\mathrm{C} 1 \mathrm{~s})^{-1} 3 \mathrm{p} \pi$ Rydherg resonances, each with a fundamental transition $(0 \rightarrow 0)$ and two vibrational sidebands. In addition, the least-squares analysis indicated the presence of a weak pair of previously unobserved transitions underlying the $3 p \pi$ states. In analogy to the NO valence and $\mathrm{N}_{2} 1 \mathrm{~s}^{-1}$ spectra, these features are assigned to the (C 1s) ${ }^{-1}$ $3 p \sigma$ state with one vibrational sideband. Franck-Condon fits of the (C 1s) $)^{-1} 3 \mathrm{~s} \sigma$ and (C $1 \mathrm{~s})^{-1} 3 \mathrm{p} \pi$ vibrational structure give larger vibrational spacings, $h v^{\prime}(3 \mathrm{~s} \sigma)=307(3) \mathrm{meV}$ and $h V^{\prime}(3 p \pi)=309(3) \mathrm{meV}$, and shorter bond lengths, $\mathrm{R}^{\prime}(3 \mathrm{~s} \sigma)=1.077(1) \AA$ and $\mathrm{R}^{\prime}(3 \mathrm{p} \pi)=$ $1.073(1) \AA$, as compared both to the ground state and the (C $1 \mathrm{~s})^{-1} \pi^{*}$ state. These results are consistent with the stronger bonding expected for excitations into the nonbonding Rydberg orbitals rather than into the antibonding $\pi^{*}$ orbital. They also indicate that the $\mathrm{C}$ 1s core hole tends to strengthen the bond, relative to the ground state. This is expected because the bonding electrons of ground-state $\mathrm{CO}$ are somewhat polarized towards the oxygen end, and the creation of the $\mathrm{C}$ 1s core hole redistributes these bonding electrons to increase the interatomic electron density. This may be seen directly by comparing the 
spatial distributions of the $1 \pi$ molecular orbitals for $\mathrm{CO}$ with those of NO (i.e. the equivalent-core molecule for $\mathrm{C}^{*} \mathrm{O}$ ). As shown in Fig. III-3 [5], these orbitals are more asymmetric in $\mathrm{CO}$ than in NO. Note that in the $(\mathrm{C} 1 \mathrm{~s})^{-1} \pi^{*}$ state, the bond-strengthening effect of the $\mathrm{C}$ is core hole is overcome by the presence of the excited electron in the strongly anti-bonding $\pi^{*}$ orbital, as demonstrated by the larger bond length and smaller vibrational spacing, as compared to the ground state.

Above $294.4 \mathrm{eV}$ there are many overlapping features, but due to the good combination of statistics and resolution a definitive analysis and assignment is possible. In particular, six electronic states are identified, most showing vibrational sidebands, as indicated in Fig. III-2 and Table III-1. A Franck-Condon analysis was performed for the $(\mathrm{C} 1 \mathrm{~s})^{-1} 3 \mathrm{~d} \pi$ and $(\mathrm{C} 1 \mathrm{~s})^{-1} 4 \mathrm{p} \pi$ states, with the results shown in Table III-2. The Rydberg formula predicts the energies of the (C $1 \mathrm{~s})^{-1} \mathrm{np} \pi$ states reasonably well, for $n>3$, with the quantum defect $\delta_{\mathrm{p} \pi}=0.75$ and the $\mathrm{C}$ is ionization threshold at $296.080 \mathrm{eV}$. For the (C $1 \mathrm{~s})^{-1} 3 \mathrm{p} \pi$ state, the corresponding quantum defect is $\delta=0.775$.

The derived vibrational spacings for the $(\mathrm{C} 1 \mathrm{~s})^{-1}$ Rydberg states are shown in Table III-2. As expected, these spacing are larger than that of the ground state, because of the bond-stregthening influence of the carbon $1 \mathrm{~s}$ core-hole. The observed values agree with the vibrational spacing recently measured for carbon $1 \mathrm{~s}$ core-ionized $\mathrm{CO}$ [at and above the energy of the (C 1s $)^{-1} \sigma^{*}$ shape resonance] [6], and also with the vibrational spacing and derived bond length measured at the carbon $1 \mathrm{~s}$ ionization threshold using zero-kineticenergy photoelectron spectroscopy [7] (see Table III-2). The similarity of the bond lengths and vibrational spacings in the core-ionized and core-excited Rydberg states demonstrates the non-bonding character of the Rydberg orbitals.

\section{III.D. Oxygen K-edge}

\section{Overview}


The photoionization spectrum of $\mathrm{CO}$ near the oxygen K-edge is shown in Fig. III-

4. The observed structure may be assigned in analogy to the carbon K-edge spectral assignment. The feature at $533-536 \mathrm{eV}$ is an intense $(\mathrm{O} / \mathrm{s})^{-1} \pi^{*}$ resonance with previously unobserved vibrational fine structure. At higher energies there are weaker transitions to ( $O$ 1s) $)^{-1}$ Rydberg states.

\section{2. $(O 1 s)^{\cdot 1} \pi^{*}$ resonance}

The $(01 \mathrm{~s})^{-1} \pi^{*}$ resonance is much wider than the corresponding resonance below the carbon K-edge, with a distinctly different fine structure. The regularly-spaced peaks within the $(\mathrm{O} 1 \mathrm{~s})^{-1} \pi^{*}$ resonance are assigned to an extensive vibrational progression, which produces the very large peak width. The individual vibrational peaks are more poorly resolved than the less extensive vibrational series of the $(C 1 \mathrm{~s})^{-1} \pi^{*}$ resonance for three reasons: 1) The instrumental resolution is larger at higher photon energies; 2) The natural linewidth of the oxygen $1 \mathrm{~s}$ core level is larger than that of the carbon $1 \mathrm{~s}$ core level: 3) The vibrational spacing is smaller in the $(\mathrm{O} / \mathrm{s})^{-1} \pi^{*}$ resonance than in the $(\mathrm{C} 1 \mathrm{~s})^{-1} \pi^{*}$ resonance.

A least-squares analysis of the fine structure yielded a vibrational spacing of $146(1)$ $\mathrm{meV}$ for the $(\mathrm{O} / \mathrm{s})^{-1} \pi^{*}$ state, remarkably less than the ground-state value, $\mathrm{hv} "=269 \mathrm{mcV}$. A Franck-Condon fit was not performed, because of difficulty in determining the energy of the fundamental transition (i.e. the band origin). The broad intensity envelope of the ( $O$ $1 \mathrm{~s})^{-1} \pi^{*}$ resonance clearly indicates, however, that there is a large change in bond length relative to the ground state of the molecule. This is in striking contrast to the very limited vibrational progression and relatively small change in bond length for the corresponding resonance at the carbon K-edge. Both transitions promote an electron into the same $\pi^{*}$ molecular orbital, thus the difference in final-state structures is attributed to the differing 
influence of the carbon and oxygen is core holes upon molecular bonding. As discussed above in Section III.C.2, the carbon core hole tends to strengthen honding by pulling bonding electron density towards the center of the molecule. The oxygen core hole has the opposite effect. i.e. bonding electron density is withdrawn from the center of the molecule. This effect, in combination with the presence of an electron in the antibonding $\pi^{*}$ orbital, results in a large decrease in hond strength for the $(\mathrm{O} / \mathrm{s})^{-1} u^{*}$ resonance as compared to the ground state.

\section{3. (O 1s) $)^{-1}$ Rydberg resonances}

The $(\mathrm{O} \text { |s })^{-1}$ Rydherg resonances shown in Fig. III.4 are assigned in analogy to the carbon K-edge spectrum of Fig. III-2. The (O Is)-1 Rydherg states have vibrational sidebands, but these features are not as well-resolved as they are in the comesponding Rydherg resonances below the carbon K-edge. This difference arises for the same reasons given above for the $(\mathrm{O} / \mathrm{s})^{-1} \pi^{*}$ resonance. Despite the poorer resolution, some FranckCondon analysis is possible. Derived parameters for the $(O / \mathrm{ls})^{-1} 3 \mathrm{~s} \sigma$ and $(\mathrm{O} / \mathrm{s})^{-1} 3 \mathrm{p} \pi$ states are $h v^{\prime}(3 s \sigma)=227(8) m e V, h v^{\prime}(3 p \pi)=224(9) m e V, R^{\prime}(3 s \sigma)=1.167(9) \AA$, and $R^{\prime}(3 p \pi)=1.159(9) \AA$. These results indicate a weakening of the hond, as compared to the ground state and the (C 1s) $)^{-1}$ Rydherg states. This is expected from the influence of the oxygen Is core hole. Note that the derived vibrational frequencies are larger for the $(\mathrm{O} / \mathrm{s})^{-1}$ Rydberg states than for the $(\mathrm{O} / \mathrm{s})^{-1} \pi^{*}$ state, indicating a stronger bond for the Rydberg states, because the Rydherg orhitals are essentially non-bonding and the $\pi^{*}$ orbital is strongly antibonding.

\section{III.E. Comparison to equivalent-core molecules}

Table III- 1 shows the derived term values for the $(\mathrm{C} \text { Is })^{-1}$ states of $\mathrm{CO}$ in 
comparison to the term values of the corresponding states in the equivalent-core molecule NO. The good agreement supports the present assignment, which in fact used this comparison as a guide. Table III-2 lists the results of Franck-Condon analyses for the various core-excited states of $\mathrm{CO}$. The derived vibrational spacings and bond lengths are compared to known values for corresponding states in the equivalent-core molecules NO and CF. The good agreement indicates the level of accuracy of the equivalent-core approximation. The largest discrepancies occur for the $\pi^{*}$ states, where the approximation is perhaps less valid because the $\pi^{*}$ orbital has appreciable amplitude near the core vacancy. (N 1 s $)^{-1}$ core-excited states of $\mathrm{N}_{2}[8]$ may also be compared to the equivalent-core NO molecule, giving slightly poorer agreement in Table III-2 than for the (C $1 \mathrm{~s})^{-1}$ core-excited states of $\mathrm{CO}$. Note that the vihrational spacings in the last column of Table III-2are corrected for differences in atomic masses.

A comparison of bond lengths and vibrational spacings in the core-excited Rydberg states to the corresponding values in the ground state indicates that the influence of the core hole upon hond strength is smaller in core-excited $\mathrm{N}_{2}$ than in core-excited $\mathrm{CO}$. Because the bonding electrons of ground-state $\mathrm{N}_{2}$ are inherently unpolarized, the redistribution of that charge due to the creation of the core hole has only a small effect on the hond strength. This contrasts with $\mathrm{CO}$, where the polarization of the ground-state bonding electrons provides a greater potential for a core-hole-induced modification of the hond strength. 


\section{REFERENCES:}

I. M. Tronc, G. C. King, and F. H. Read, J. Phys. B 12, 137 (1979).

2. D. A. Shaw, G. C. King, D. Crejanovic, and F. H. Read, J. Phys. B 17, 2091 (1984).

3. A. P. Hitcheock and C. E. Brion, J. Electr. Spectrosc.18, 1 (1980).

4. K. P. Huber and G. Herzberg. Molecular Spectra and Molecular Structure, Vol. 4 (Van Nostrand Reinhold, New York, 1979).

5. W. L. Jorgensen and L. Salem. The Organic Chemist's Book of Orbirals, (Academic Press, New York, 1973).

6. K. J. Randall, A. L. D. Kilcoync, H. M. Kuppe, J. Feldhaus, A. M. Bradshaw, J.-E. Rubensson, W. Eberhardt, Z. Xu, P. D. Johnson, and Y. Ma, to be published.

7. L. J. Medhurst, P. A. Heimann, M. R. F. Siggel, D. A. Shirley, C. T. Chen, Y. Ma. S. Modesti, and F. Sette. Chem. Phys. Lett. 193, 493 (1992).

8. C. T. Chen, Y. Ma, and F. Selle, Phys. Rev. A40, 6737 (1989). 
Table III-1: Energies and assignments of the (C $1 \mathrm{~s})^{-1}$ core-excited states and their vibrational sidebands. Uncertainties in the units of the last digit are given in parenthesis, based upon an assumed value of $287.400 \mathrm{eV}$ for the $(\mathrm{C} 1 \mathrm{~s})^{-1} \pi^{*}, v^{\prime}=0$ resonance. In the last two columns, the term values for the (C $1 s)^{-1}$ states and the equivalent-core $2 \pi$-excited states of NO are compared. All energies are in eV.

\begin{tabular}{|c|c|c|c|c|c|c|}
\hline & \multirow[t]{2}{*}{$v^{\prime}=0$} & \multirow[t]{2}{*}{$v^{\prime}=1$} & \multirow[t]{2}{*}{$v^{\prime}=2$} & \multirow[t]{2}{*}{$v^{\prime}=3$} & \multicolumn{2}{|c|}{ Term values } \\
\hline & & & & & $\mathrm{CO}^{\mathrm{a}}$ & NOb \\
\hline \multicolumn{7}{|c|}{$(\mathrm{C} / \mathrm{s})^{-1}$} \\
\hline$\pi^{*}$ & $287.400)$ & $287.656(2)$ & $287.912(2)$ & $288.168(3)$ & 8.680 & 9.264 \\
\hline $3 \mathrm{~s} \sigma$ & $292.368(2)$ & $292.665(2)$ & $292.956(2)$ & & 3.712 & 3.799 \\
\hline $3 \mathrm{p} \pi$ & $293.331(1)$ & $293.630(2)$ & $293.922(2)$ & & 2.749 & 2.786 \\
\hline 3po & $293.499(3)$ & $293.787(4)$ & & & 2.581 & 2.672 \\
\hline $3 \mathrm{~d} \pi$ & $294.614(2)$ & $294.920(5)$ & $295.226(6)$ & & 1.466 & 1.503 \\
\hline $4 \mathrm{p} \pi$ & $294.803(2)$ & $295.090(2)$ & $295.374(5)$ & & 1.277 & 1.309 \\
\hline $4 \mathrm{~d} \pi$ & $295.275(5)$ & $295.56(1)$ & & & 0.805 & 0.863 \\
\hline $5 p \pi$ & $295.345(3)$ & $295.635(5)$ & & & 0.735 & 0.768 \\
\hline $6 \mathrm{p} \pi$ & $295.561(5)$ & $295.866(7)$ & & & 0.519 & 0.508 \\
\hline $7 p \pi$ & $295.744(9)$ & & & & & \\
\hline
\end{tabular}

a Relative to the $\mathrm{C}$ 1s binding energy $(296,080 \mathrm{eV}$, determined by the Rydherg analysis). b Relative to the $2 \pi$ binding energy (Ref. $[4]$ ). 
Table III-2: Equilibrium distances R, vibrational energies hv, and anharmonicity constants $h v x$ for $\mathrm{CO}$ (this work) and $\mathrm{N}_{2}[8]$ in the ground state and in core-excited states.

Uncertainties in the units of the last digit are given in parenthesis. The parameters in the last two columns are taken from ref. [4] and the hv values were corrected for the differences in reduced masses (multiplied by $[16 / 15]^{1 / 2},[49 / 45]^{1 / 2}$, and $[133 / 124]^{1 / 2}$ for $\mathrm{NO} \rightarrow \mathrm{N}_{2}^{*}, \mathrm{NO} \rightarrow \mathrm{C}^{*} \mathrm{O}$, and $\mathrm{CF} \rightarrow \mathrm{CO}^{*}$, respectively).

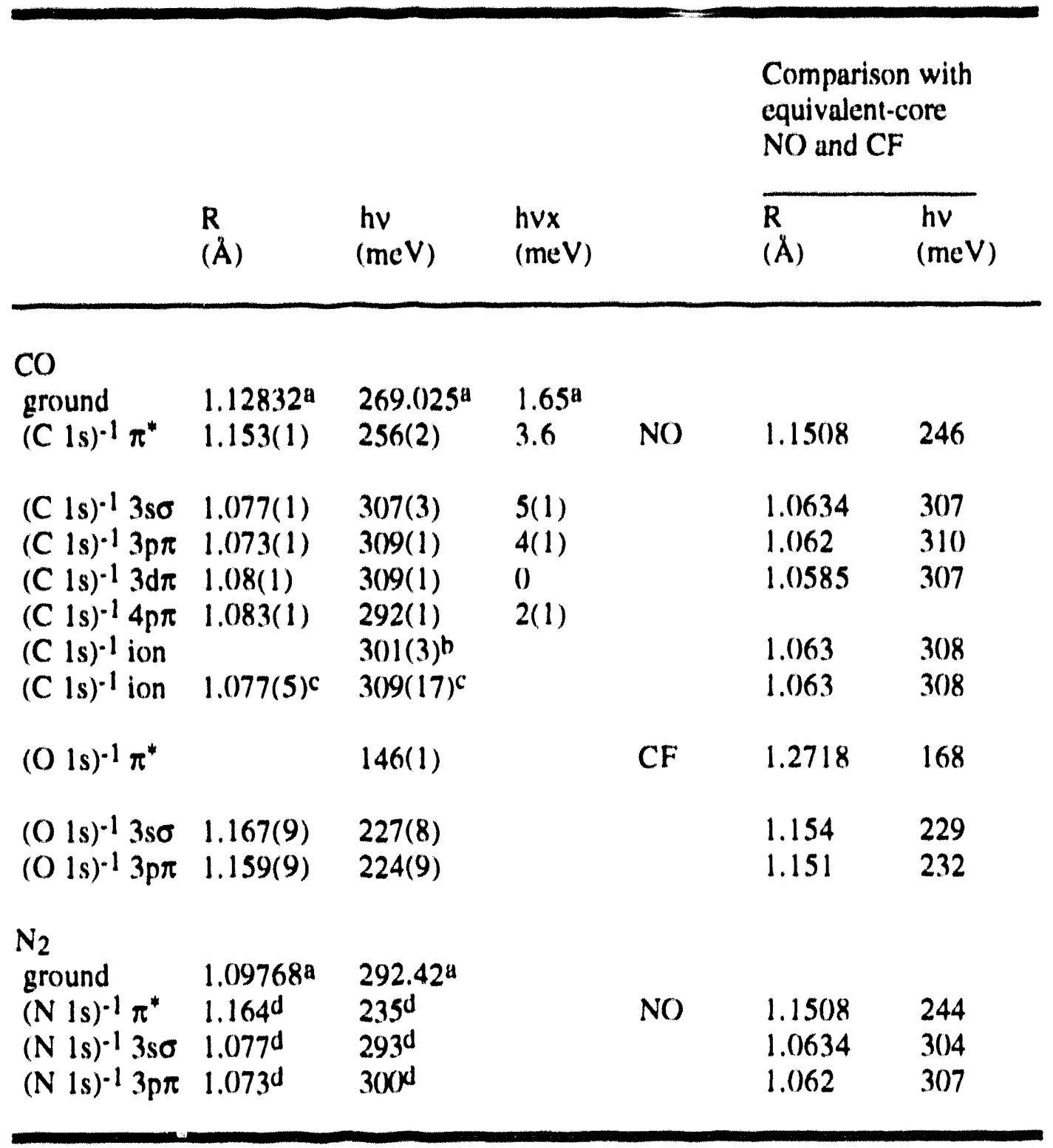

aRef. [4]. bRef. [6]; measured by photoelectron spectroscopy at $\mathrm{KE}=7.5$ and $16 \mathrm{eV}$, i.e. at the energy of the $\sigma^{*}$ shape resonance, and also somewhat higher. cRef. [7]; measured by zero-kinetic-energy photoelectron spectroscopy. dRef. [8]. 


\section{FIGURE CAPTIONS:}

Figure III-1: The photoabsorption spectrum of gas-phase $\mathrm{CO}$ near the carbon $\mathrm{K}$ edge. Note the vertical scale change, which multiplies intensity in the region $292-310 \mathrm{eV}$ by a factor of 10 relative to the lower range $287-292 \mathrm{eV}$, and also shifts the baseline. The inset shows the $(\mathrm{C} 1 \mathrm{~s})^{-1} \pi^{*}$ resonance with an expanded energy scale, to better display the vibrational sidebands and the results of a least-squares Franck-Condon fit to the data.

Figure III-2: An expanded view of the Rydberg region of Fig. III-1, showing the (C 1s) ${ }^{-1}$ Rydberg states of $\mathrm{CO}$. Assignment bars indicate the seven vibrational progressions which are resolved. The results of least-squares Franck-Condon fits for each progression are plotted with the data. Note the vertical scale change at $294.4 \mathrm{eV}$, which doubles the intensity of the data in the upper range and shifts the baseline.

Figure III-3: Spatial distribution of the $1 \pi$ molecular orbital in ground-state $\mathrm{CO}$ and NO. Reprinted from Ref. [5] with permission.

Figure III-4: The photoabsorption spectrum of CO near the oxygen K-edge. Note the vertical scale change, which multiplies intensity by a factor of 10 in the upper range and also shifts the baseline. Assignment bars and the results of a least-squares Franck-Condon analysis are shown with the data. The weak but distinct vibrational structure within the $(\mathrm{O}$ $1 \mathrm{~s})^{-1} \pi^{*}$ resonance is observed here for the first time. 


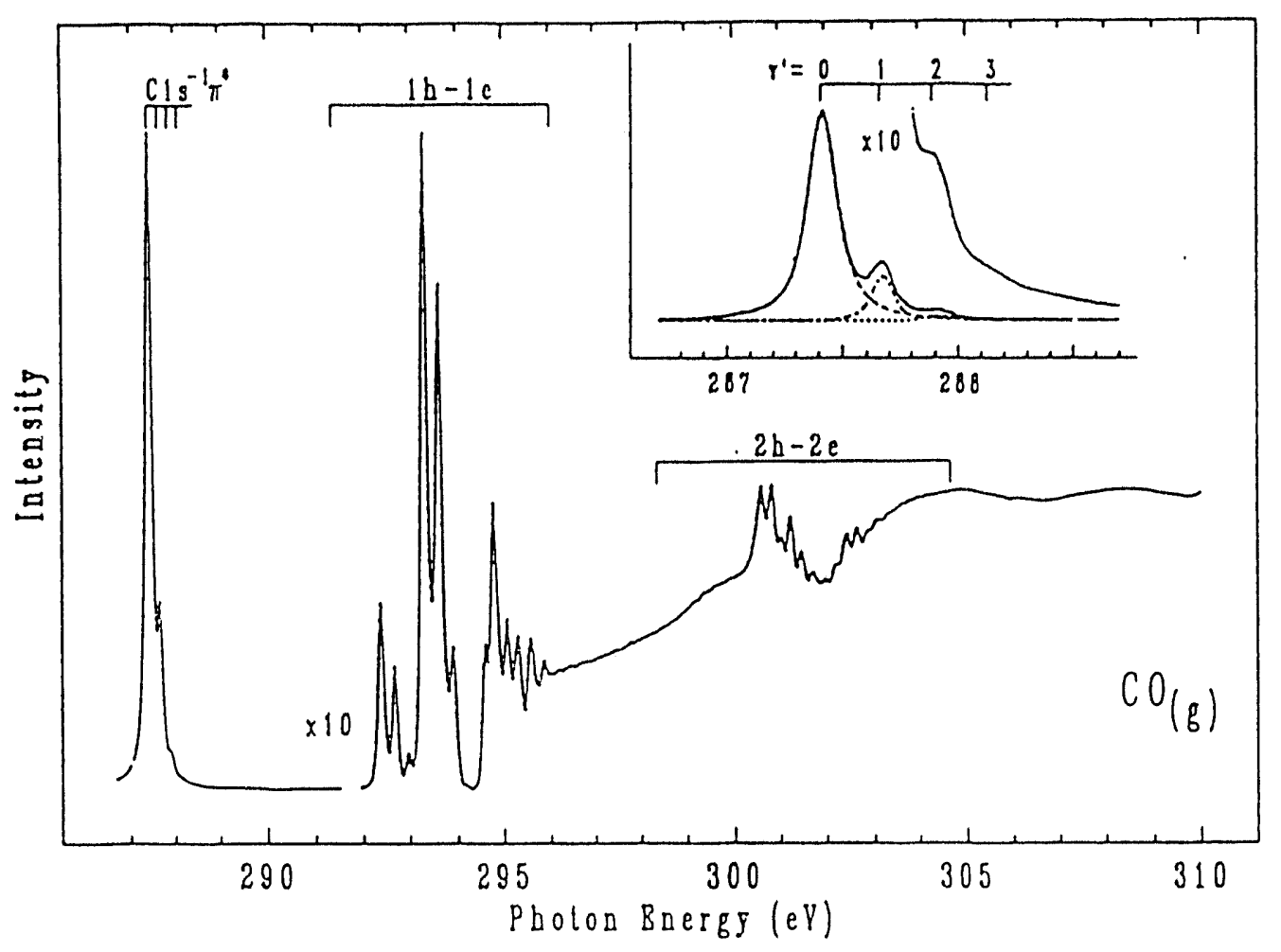

Figure III-1 


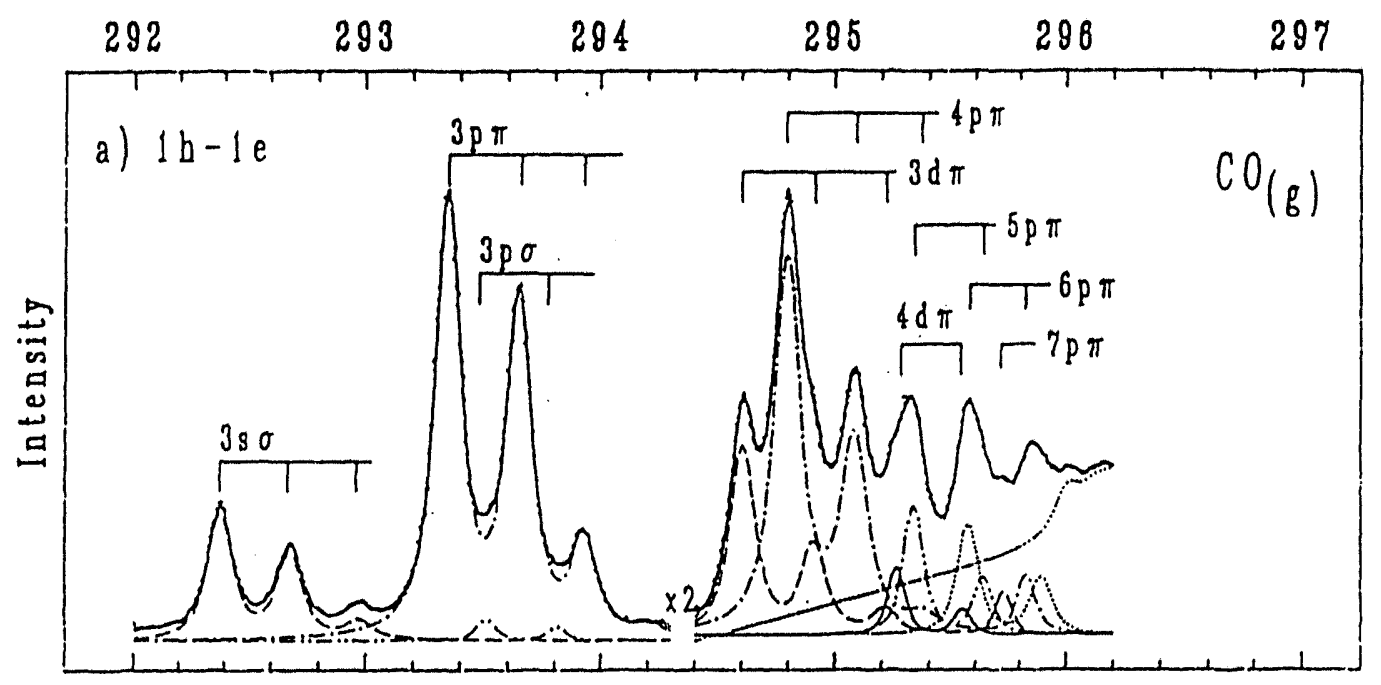

Figure III-2 

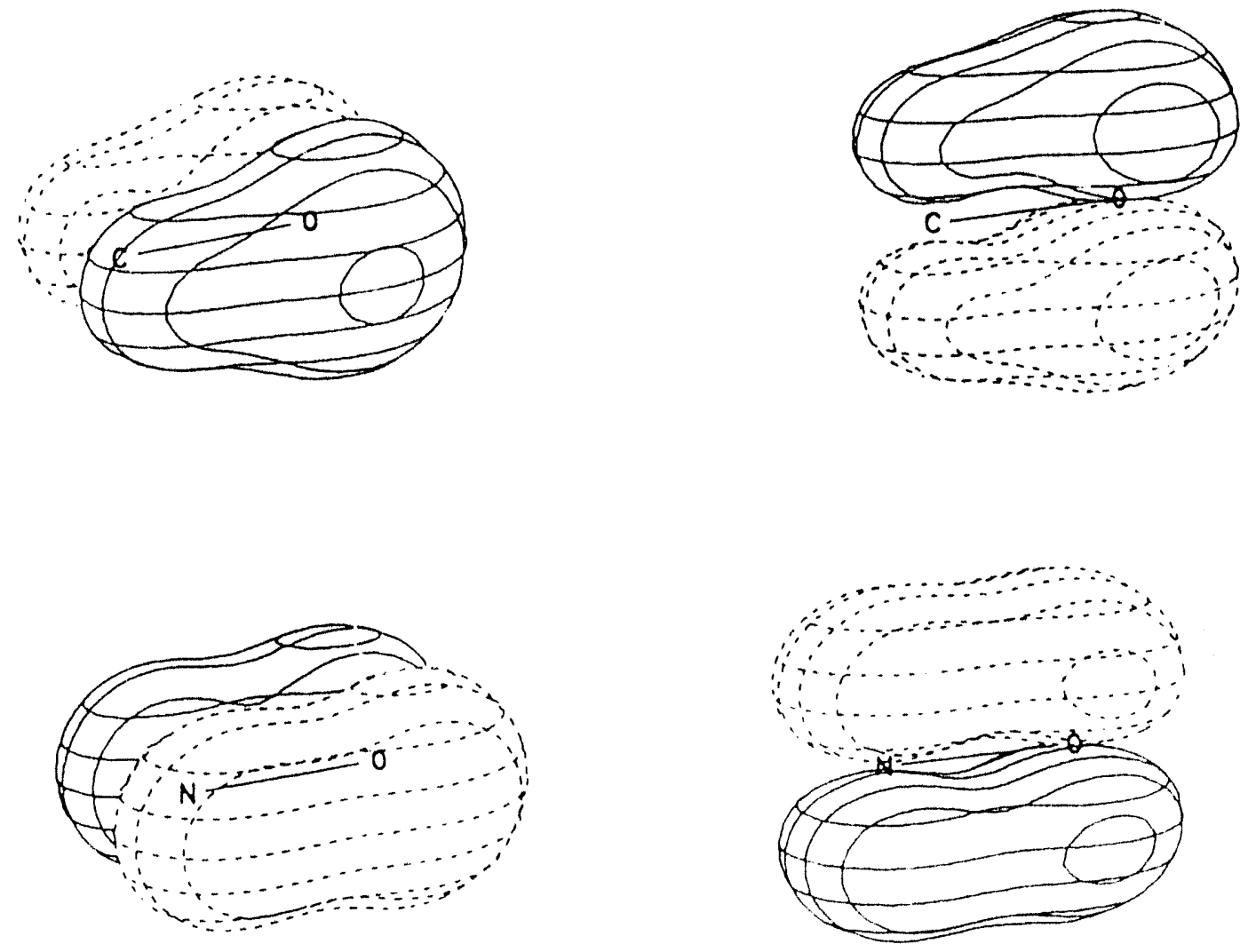

Figure III-3 


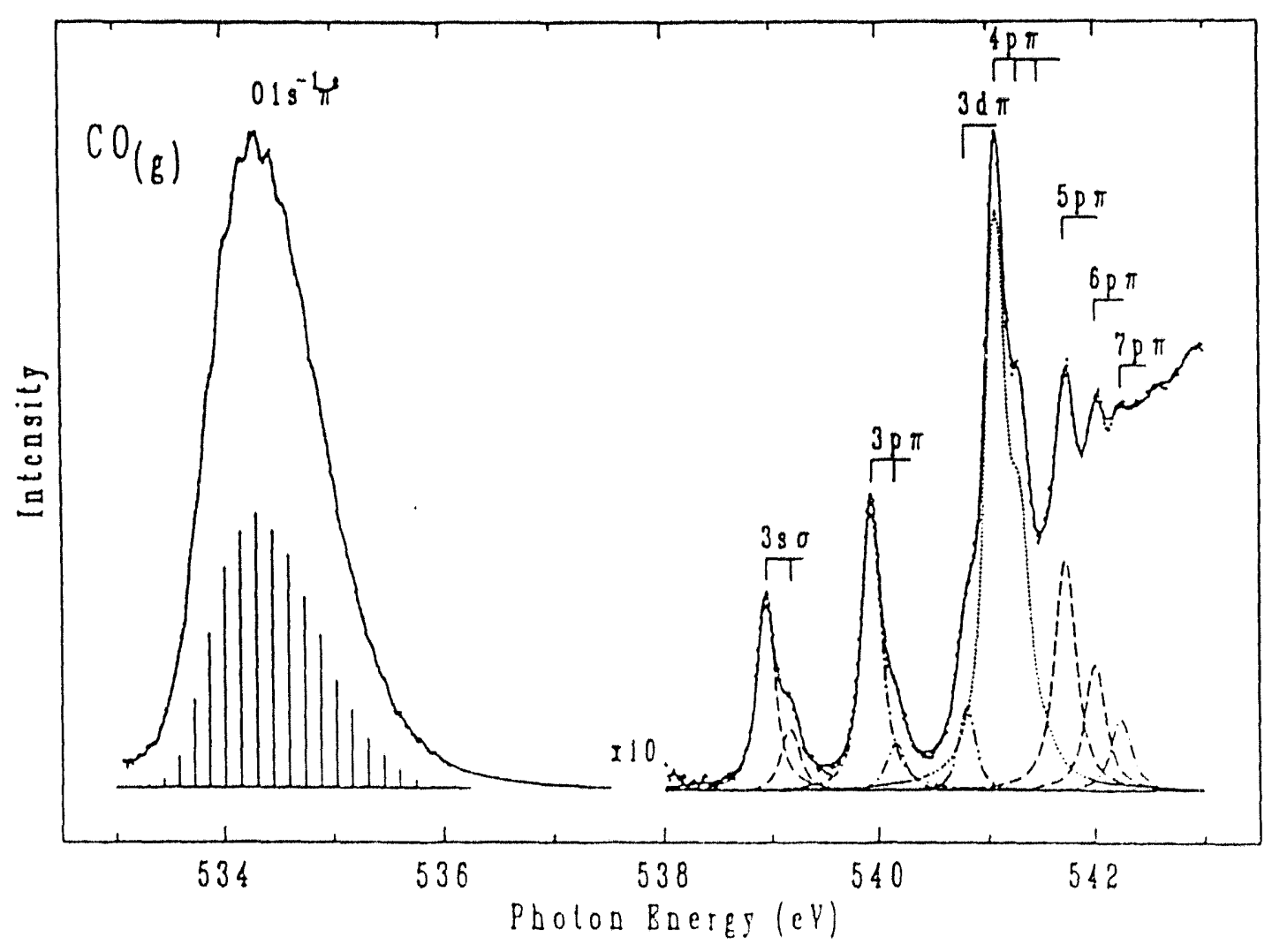

Figure III-4 


\section{CHAPTER IV: HIGH-RESOLUTION PHOTOIONIZATION OF FORMALDEHYDE AT THE CARBON AND OXYGEN K- EDGES}

\section{IV.A. Introduction}

Limitations in spectral resolution and/or counting rates have restricted the study of vibrational features in the core-excited electronic states of molecules. In particular, vibrational fine structure in XANES has been examined mainly for diatomic molecules; few polyatomic systems have been fully characterized. For formaldehyde, $\mathrm{H}_{2} \mathrm{CO}$, excitations of the carbon $1 s$ and oxygen 1 s electrons into $\pi^{*}$ and Rydberg orbitals were studied by EELS with a resolution of $\approx 250 \mathrm{meV}$ [1], providing only an approximate understanding of these core-excited states. The EELS spectra were compared to the results of theoretical calculations [2], but several questions remained unresolved. Because $\mathrm{H}_{2} \mathrm{CO}$ is a four-atom molecule with six vibrational modes, a relatively complicated fine structure is expected; excitations of various combinations of vibrational modes can produce highly structured spectra, particularly below the carbon $\mathrm{K}$ edge.

The present study benefits from both higher energy resolution and lower noise levels, as compared to the EELS results. The carbon and oxygen K-edge photoionization spectra of $\mathrm{H}_{2} \mathrm{CO}$ and, for the first time, $\mathrm{D}_{2} \mathrm{CO}$, have been measured. For the prominent $(\mathrm{C} 1 \mathrm{~s})^{-1} \pi^{*}$ resonance, vibrational fine structure is resolved and assigned to the $\mathrm{C}-\mathrm{O}$ and symmetric $\mathrm{C}-\mathrm{H}$ stretch modes as well as the $\mathrm{CH}_{2}$ scissors bend mode. Several of the more intense (C 1s) $)^{-1}$ Rydberg states also exhibit vibrational excitation of these two stretch modes. For deuterated formaldehyde, $\mathrm{D}_{2} \mathrm{CO}$, isotopic shifts in vibrational spacings and spectral intensities are clearly observed in the vibrational sidebands. Equilibrium bond lengths and bond angles are derived from Franck-Condon analyses of vibrational fine structures. Below the carbon 1 s ionization threshold, Rydberg states up to $n=9$ are 
assigned. Striking isotopic shifts effects are also observed in the energy and linewidth of the ground vibrational level of the 3s-a 1 Rydberg state, for both carbon 1s and oxygen is excitations. These shifts are tentatively attributed to large changes in the vibrational zeropoint energy upon excitation, perhaps indicating a dissociative state. Similar effects in the carbon K-edge spectra of several other molecules are discussed.

\section{IV.B. Experimental}

These measurements utilized the 2442 line/mm grating on the SX700/1I monochromator. In the first order of diffraction, the spectral resolution was $=60 \mathrm{meV}$ (FWHM) at the carbon K-edge and $\approx 150 \mathrm{meV}$ at the oxygen K-edge. The photon energy was calibrated using the (C $1 \mathrm{~s})^{-1} \pi^{*}, v^{\prime}=0$ resonance of gas-phase $\mathrm{CO}$ (at hv $=287.40 \mathrm{eV}$ ) and the $\left(\mathrm{N}_{1 \mathrm{~s}}\right)^{-1} \pi^{*}, v^{\prime}=0$ resonance of gas-phase $\mathrm{N}_{2}$ (at hv $=400.88 \mathrm{eV}$ ) [3]. Gas-phase $\mathrm{H}_{2} \mathrm{CO}$ and $\mathrm{D}_{2} \mathrm{CO}$ were produced by thermal decomposition of the corresponding paraformaldehyde at $\approx 70^{\circ} \mathrm{C}$. Sample pressures of $0.06-0.08 \mathrm{mbar}$ in the gas cell were separated from the monochromator by a $1500 \AA \mathrm{Al}(1 \% \mathrm{Si})$ window.

\section{IV.C. Carbon K-edge}

\section{Overview}

Fig. IV-1 shows an overview photoionization spectrum of $\mathrm{H}_{2} \mathrm{CO}$ in the region of the carbon $1 \mathrm{~s}$ ionization threshold. The $(\mathrm{C} 1 \mathrm{~s})^{-1} \pi^{*}$ resonance is observed between 285 and $287 \mathrm{eV}$. The spectrum shows a series of peaks between $\approx 290 \mathrm{eV}$ and the carbon $\mathrm{K}$ edge at $\approx 294.5 \mathrm{eV}$. These arise from the promotion of the carbon $1 \mathrm{~s}$ electron into Rydberg orbitals. The inset shows a very broad resonance $\approx 15 \mathrm{eV}$ above threshold; this is assigned to a $\sigma^{*}$ shape resonance. The weaker broad feature at $\approx 301 \mathrm{eV}$, indicated by the dashed 
vertical bar, is tentatively assigned to multielectron excitations.

\section{2. (C $1 \mathrm{~s})^{-1} \pi^{*}$ resonance}

In the EELS measurement, the (C $1 s)^{-1} \pi^{*}$ resonance was observed as a relatively broad feature at $\approx 286 \mathrm{eV}$, with a small shoulder on the low-energy side [1]. In contrast, the present high-resolution spectra reveal detailed fine structure. The (C $1 \mathrm{~s})^{-1} \pi^{*}$ resonances for $\mathrm{H}_{2} \mathrm{CO}$ and $\mathrm{D}_{2} \mathrm{CO}$ are shown in more detail in Fig. IV-2. The distinctive peaks and shoulders, with isotopic shifts, allow for the first time a detailed analysis of the many transitions involved.

The designation of this state as a $\pi^{*}$ resonance is made in analogy to the corresponding resonance in core-excitation spectra of diatomic molecules. In the $\mathrm{C}_{2 v}$ symmetry of the formaldehyde molecule, the $\pi^{*}$ orbital loses its degeneracy and splits into two components. The lower level has b symmetry and is occupied in the ground electronic state [2]. Thus, the resonances observed here correspond to transitions into the unoccupied orbital of $b_{1}$ symmetry. Having established the exact symmetry of this resonance, the commonly used " $\pi^{*}$ " designation will be used henceforth.

To interpret vibrationally resolved spectra of polyatomic molecules, a normal coordinate analysis is essential. In the present analysis, the approach described by Wilson, Decius, and Cross [4] was applied. In the space of internal coordinates, which conveniently represents internuclear distances and bond angles, the $\mathrm{G}$ matrix (derived from the atomic masses and molecular geometry) and the F matrix (which contains the force constants) were constructed. The values of the force constants were taken from Ref. [5]. The internal coordinates were transformed into symmetry coordinates (symmetric $\mathrm{C}-\mathrm{H}$ stretch, $\mathrm{C}-\mathrm{O}$ stretch, $\mathrm{HCH}$ scissors bend, etc.) and then to normal coordinates by simultaneously diagonalizing the $\mathrm{F}$ and $\mathrm{G}$ matrices. The contributions of the symmetry coordinates to the normal coordinates was thereby obtained. Some of the normal 
coordinates have significant contributions from more than one symmetry coordinate. Thus, the commonly used designations [6] for the $v_{1}, v_{2}$, and $v_{3}$ vibrational modes as $C \cdot H$ symmetric stretch, C-O stretch, and $\mathrm{HCH}$ scissors bend, respectively, are only approximate, as expected. Moreover, the contributions of the symmetry coordinates to a given normal coordinate depend on mass and geometry; thus they will be different for $\mathrm{H}_{2} \mathrm{CO}$ and $\mathrm{D}_{2} \mathrm{CO}$.

An unambiguous fit of the $(C 1 s)^{-1} \pi^{*}$ resonance is difficult to achieve, because strong excitations of three of the six normal modes, in any combination, are dipole-allowed for this transition. The present fit takes all three of these totally-symmetric modes into account. They are the $v_{1}$ mode (mainly symmetric $\mathrm{C}-\mathrm{H}$ stretch), the $\mathrm{v}_{2}$ mode (mainly $\mathrm{C}-\mathrm{O}$ stretch), and the $v_{3}$ mode (commonly attributed to $\mathrm{HCH}$ scissors bend, but consisting in reality of mainly $\mathrm{C}-\mathrm{O}$ stretch with $=30 \% \mathrm{HCH}$ bend). These normal modes have groundstate vibrational energies of $h v_{1}=345.0 \mathrm{meV}, h v_{2}=216.5 \mathrm{meV}$, and $h v_{3}=186.0 \mathrm{meV}$ for $\mathrm{H}_{2} \mathrm{CO}[6]$ and $h v_{1}=254.9 \mathrm{meV}, h v_{2}=211.0 \mathrm{meV}$, and $h v_{3}=137.1 \mathrm{meV}$ for $D_{2} C O[6]$. The isotopic differences in ground-state vibrational energies are largest for $v_{1}$ and smallest for $V_{2}$, which confirms the assignments of these normal modes to predominantly C-H and C-O stretch modes, respectively. Results of the least-squares fit are plotted in Fig. IV-2 with the data, and discussed in Section IV.C.4.

The first peak, at $h v=285.59 \mathrm{eV}$, is caused by a transition from the $v^{\prime \prime}=(0,0,0)$ level of the electronic ground state $\left[v^{\prime \prime}=(0,0,0)\right.$ denotes the vibrational ground level: $\left.v_{1} \prime=0, v_{2}^{\prime \prime}=0, v_{3} \prime=0\right]$ to the $v^{\prime}=(0,0,0)$ level of the $(\mathrm{C} 1 \mathrm{~s})^{-1} \pi^{*}$ electronic state. As usual, the single prime indicates vibrational parameters for the electronically excited state, the double prime applies to the ground electronic state, and $v_{q}$ is the quantum number for the $\mathrm{i}^{\text {th }}$ vibrational mode. At higher photon energies, transitions are visible which include the excitation of quanta of vibrational energy in one or more of the normal modes. The subspectra of Fig. IV-2 show the $v_{2}{ }^{\prime}$ vibrational series for different fixed $v_{1}{ }^{\prime}$ and $v_{3}{ }^{\prime}$ quantum numbers. The solid subspectrum represents only the $v_{2}^{\prime}$ ("C-O stretch") series 
$(0,0,0),(0,1,0),(0,2,0),(0,3,0)$, etc.; the dotted subspectrum stands for the $v_{3}^{\prime}=1$ series $(0,0,1),(0,1,1),(0,2,1),(0,3,1)$, etc. For reasons of clarity, the subspectra of the higher series show the sum of $v_{2}$ ' and $v_{3}$ ' transitions: The dashed subspectrum represents the $v_{1}^{\prime}=1$ series with combinations of $v_{2}^{\prime}$ and $v_{3}^{\prime}$ excitations $(1,0,0),(1,1,0)+(1,0,1)$, $(1,0,2)+(1,1,1)+(1,2,0)$, etc.; while the dash-dotted and dash-double-dotted ones are the $v_{1}^{\prime}=2$ series $(2,0,0),(2,1,0)$, etc.; and the $v_{1}^{\prime}=3$ series $(3,0,0),(3,1,0)$, etc.; respectively.

Tuming from the $\mathrm{H}_{2} \mathrm{CO}$ to the deuterated form, $\mathrm{D}_{2} \mathrm{CO}$, a substantial change in the vibrational fine structure of the (C $1 \mathrm{~s})^{-1} \pi^{*}$ resonance is observed (see Fig. IV-2). It is remarkable that this change occurs not only in the higher photon-energy region, for excitations of the $v_{1}$ normal mode, which is usually assigned to the symmetric $\mathrm{C} \cdot \mathrm{H}$ stretch. but also in the low-energy region, where mainly the $v_{2}$ mode is excited. In particular, the intensity ratio of the first two peaks is clearly different between the two isotopic species. The second peak is assigned to the $(0,1,0)$ vibrational level, and the $v_{2}$ mode is primarily composed of the $\mathrm{C}-\mathrm{O}$ stretch. This observation indicates that, upon excitation of the (C 1s) $)^{-1} \pi^{*}$ resonance, the $\mathrm{C}-\mathrm{O}$ hond length changes more in the case of $\mathrm{H}_{2} \mathrm{CO}$ than for $D_{2} C O$. (The isotopic effect upon this intensity ratio may also result from the differing $v_{2}$ vibrational energies in the two isotopic species, but clearly this difference is small in this case.) Isotopic shifts of the vibrational energies are indicated in Fig. IV-2 for only three selected vibrational states: the $(0,0,1)$ state by dotted vertical lines, the $(1,0,0)$ state by dashed vertical lines, and the $(2,0,0)$ state by dash-dotted vertical lines.

\section{3. (C 1s) $)^{-1}$ Rydberg resonances}

Fig. IV-3 shows the transitions to the (C 1s) $)^{-1}$ Rydherg excited states in $\mathrm{H}_{2} \mathrm{CO}$ and $\mathrm{D}_{2} \mathrm{CO}$. A comparison of the $\mathrm{H}_{2} \mathrm{CO}$ and $\mathrm{D}_{2} \mathrm{CO}$ spectra immediately distinguishes fundamental transitions to Rydberg states (i.e. without vibrational excitation) from 
vibrational sidebands (i.e. including excitation of vibrations in the final state).

Fundamental transitions will exhibit no isotopic energy shift, whereas vibrational sidebands will shift to lower energies in the deuterated species. The only exception to this rule in the present work is found for the $(\mathrm{C} / \mathrm{s})^{-1} 3 \mathrm{~s}-\mathrm{a}_{1}$ Rydherg state, where an isotopic energy shift is observed even for the fundamental transition (see the dash-dotted subspectra in Figs. IV. 3(a) and (b), and the discussion below). Solid subspectra lines are used in Fig. IV-3 to represent the fundamental peaks, i.e. transitions to the ground vibrational level of core. excited Rydberg states.

Rydherg series in core-excited molecules are normally assigned on the hasis of their quantum defects as well as by comparison with known series in the valence-excited equivalent-core molecule. Using the $\mathrm{Z}+1$ approximation, the equivalent-core molecule of (C 1s) $)^{-1} \mathrm{H}_{2} \mathrm{CO}$ is $\mathrm{H}_{2} \mathrm{NO}$. Since litule data is available for $\mathrm{H}_{2} \mathrm{NO}$, a comparison to the $(\mathrm{C}$ 1s) ${ }^{-1}$ Rydherg states of $\mathrm{CO}[7]$ is used here instead. In the two molecules, $\mathrm{CO}$ and $\mathrm{H}_{2} \mathrm{CO}$. the $\mathrm{C}$. $\mathrm{O}$ bonds are fairly similar, with the iwo $\mathrm{H}$ atoms in $\mathrm{H}_{2} \mathrm{CO}$ expected to influence mainly the intensities and vibrational sidebands of the Rydherg transitions. This leads to the assignments given in Fig. IV-3 and in Table IV-I for hoth $\mathrm{H}_{2} \mathrm{CO}$ and $\mathrm{D}_{2} \mathrm{CO}$. Table IV1 also provides a comparison of the present results for $\mathrm{H}_{2} \mathrm{CO}$ with those of other measurements and of theoretical calculations. A number of previously unresolved Rydherg states were identified in the present work and will be discussed in more detail helow.

The first relatively broad feature at hv $=290) .2 \mathrm{eV}$ arises from the transition to the (C 1s) ${ }^{-1} 3 \mathrm{~s}$-a core-excited Rydberg state, which shows a high-energy shoulder due to vibrational excitations. This resonance can he compared to the $(\mathrm{C} \text { 1s })^{-1} 3$ so state in $\mathrm{CO}$ [7]. The natural width of this peak, derived from a least-squares fit. is $240 \mathrm{meV}$ (FWHM), which is much larger than the derived widths of the $(\mathrm{C} 1 \mathrm{~s})^{-1} \pi^{*}$ states and of the higher Rydberg states in $\mathrm{H}_{2} \mathrm{CO}$. The (C $\left.1 \mathrm{~s}\right)^{-1} 3 \mathrm{~s}-\mathrm{a}_{1}$ state of formaldehyde is also broader than all the $(\mathrm{C} / \mathrm{s})^{-1}$ states in $\mathrm{CO}$ (including the $(\mathrm{C} / \mathrm{s})^{-1} 3 \mathrm{~s} \sigma$ state). Although the $(\mathrm{C} \mid \mathrm{s})^{-1} 3 \mathrm{~s}-\mathrm{a}_{1}$ transition was previously observed in the EELS study [1], it did not appear any broader 
than the higher Rydberg excitations due to the lower energy resolution of the EELS measurements. This broadening is accompanied by a distinctive isotopic shift of the energy of this peak, which changes from $290.178 \mathrm{eV}$ for $\mathrm{H}_{2} \mathrm{CO}$ to $290.232 \mathrm{eV}$ for $\mathrm{D}_{2} \mathrm{CO}$. The change in energy is indicated in Fig. IV-3 by vertical hars. A close inspection of Fig. IV-3 shows that an isotopic effect is also observed for the width of the 3s-a, Rydberg state. The natural linewidth, determined from a least-squares analysis, is only $180 \mathrm{meV}$ for $\mathrm{D}_{2} \mathrm{CO}$ as compared to $240 \mathrm{meV}$ for $\mathrm{H}_{2} \mathrm{CO}$. These effects will be discussed in more detail in Sec. IV.E, in conjunction with the similar effects for the $(\mathrm{O} 1 \mathrm{~s})^{-1} 3 \mathrm{~s}-\mathrm{a}$ ) and $\left(2 b_{1}\right)^{-1} 3 \mathrm{~s}$-a 1 states of formaldehyde and for the $(C / \mathrm{s})^{-1} 3 \mathrm{~s}$ state of other molecules. Among the higher Rydherg states, both the $3 p$ and $4 p$ states exhibit a splitting into $h_{2}$ and $b_{1}$ component levels, caused by the removal of degeneracy of the $p$ states in $C_{2 v}$ symmetry. Note that the molecular coordinates are chosen with the $x$ axis perpendicular to the molecular plane, according to the nomenclature of Ref. [8]. Actually, a molecular-field splituing into three states $\left(a_{1}, b_{1}\right.$, and $\left.b_{2}\right)$ is expected, but theoretical predictions put the (C $1 \mathrm{~s})^{-1} 3 \mathrm{p}$-a state only $100 \mathrm{meV}$ higher than the (C 1s) $)^{-1} 3 \mathrm{p}-\mathrm{b}_{2}$ state, with an oscillator strength only $-1 \%$ that of the $(\mathrm{C} 1 \mathrm{~s})^{-1} 3 \mathrm{p}-\mathrm{b}_{2}$ state $[2,9]$. Thus the $(\mathrm{C} 1 \mathrm{~s})^{-1} 3 \mathrm{p}-\mathrm{a}_{1}$ state is not expected to be observed, and there is no indication of it in the data. The $b_{2}-b_{1}$ splitting is largest for the (C $1 \mathrm{~s})^{-1} .3 \mathrm{p} \mathrm{state}(476 \mathrm{meV})$, such that the two $3 \mathrm{p}$ peaks were already resolved as separate peaks in the lower-resolution EELS work (1) and tentatively assigned as in the present study. Because neither peak shows an isotopic energy shift, an alternative assignment of the $(\mathrm{C} \text { Is })^{-1} 3 \mathrm{p}-\mathrm{b}_{1}$ peak at $\mathrm{hv}=291.73 \mathrm{eV}$ to a $\mathrm{C} \cdot \mathrm{H}$ symmetric stretch vibrational excitation of the (C 1s) ${ }^{-1} 3 \mathrm{p}$-b2 state can now he excluded, confirming the adopted assignment.

The molecular-field splitung of the $4 p$ states is naturally smaller ( $107 \mathrm{meV})$ because these orbitals have a larger spatial distribution and thus feel a weaker anisotropic field as compared to the $3 p$ states. The two features observed are again assigned to the $b_{2}$ and $b_{1}$ components, in analogy to the 3p states. However, the theoretical predictions for the (C 
1s) $)^{-1} 4 \mathrm{p} \mathrm{states}[2]$ differ from those for the (C $\left.1 \mathrm{~s}\right)^{-1} 3 \mathrm{p}$ states, suggesting the alternative assignment that the ohserved splitung arises from the $b_{2}$ and $a_{1}$ components. For the higher $p$ states, a molecular-field splitting is no longer resolved. Fig. IV-3 shows that the (C 1s) $)^{-1}$ np Rydberg series, converging at the carbon $1 s$ ionization threshold, IPC.K, is resolved up to $n=7$ and fitted up to $n=9$. A least-squares fit of the spectrum of Fig. IV-3 with the Rydherg formula leads to a carbon 1s ionization threshold of IPC.K $=294.352 \mathrm{eV}$ and a quantum defect of $\delta_{p}=1.08$. The fit results are shown by the solid line through the data points in Fig. IV-3. In analogy to the corresponding spectrum for CO [7], transitions to (C1s) $)^{-1}$ nd Rydberg states can also be identified in the spectra of Fig. IV-3. An additional excitation is observed at an energy of $292.2 \mathrm{eV}$. which can be assigned to a (C 1s) $)^{-1} 4 \mathrm{~s}$ state and its vibrational sidebands; this Rydberg state was not observed for $\mathrm{CO}$ [7]. The present assignments differ from those of Ref. (1), particularly for the $3 \mathrm{~d}$ and $4 \mathrm{~s}$ Rydberg states. The quantum defects derived from the present assignment, $\delta_{s}=1.4$ and $\delta_{\mathrm{d}}=0.05$, appear to be more reasonable. The more recent theoretical predictions for the energy of the (C $1 \mathrm{~s})^{-1} 4 \mathrm{~s}$ state, relative to the calculated energies of the other Rydherg states [9], also tend to support the present assignment (see Table IV-1). Calculations of the energies of the $(\mathrm{C} \text { /s })^{-1}$ nd Rydherg states would be useful to better evaluate the present assignment.

Particularly for the lower Rydherg states, clearly resolved vibrational sidebands are observed which are easily identified by their strong isotopic energy shifts (see dotted and dashed subspectra in Fig. IV-3). The isotopic effects on vibrational spacings are indicated by the vertical-bar diagrams in Fig. IV-3. Note also the isotopic dependence of the relative intensities of the vibrational excitations. As in the case of the (C $1 \mathrm{~s})^{-1} \pi^{*}$ resonance, more than one vibrational mode contributes to each set of fine structure. This is most easily seen for the (C $1 \mathrm{~s})^{-1} 3 \mathrm{p}-\mathrm{b}_{2}$ and $(\mathrm{C} / \mathrm{s})^{-1} 3 \mathrm{p}-\mathrm{b}_{1}$ Rydberg states, where the vibrational sidebands are clearly characterized by two different vibrational spacings. In contrast to the (C $1 \mathrm{~s})^{-1}$ $\pi^{*}$ resonance, only two vibrational modes are observed for the Rydherg states; the lower- 
energy $v_{2}$ mode (mainly C-O stretch) and the higher-energy $v_{1}$ mode (mainly $\mathrm{C}-\mathrm{H}$ symmetric stretch). It is striking that the $(C 1 s)^{-1} 3 p-b_{2}$ state, for both $\mathrm{H}_{2} \mathrm{CO}$ and $\mathrm{D}_{2} \mathrm{CO}$, shows a relatively more intense $v_{2}$ vibrational sideband, as compared to the $(C 1 s)^{-1} 3 p-b_{1}$ state (see Fig. IV-3, dotted components).

\section{Franck-Condon analysis}

For the quantitative analysis of the vibrational structure in the (C $1 \mathrm{~s})^{-1} \pi^{*}$ and (C 1s) $)^{-1}$ Rydberg resonances, a Franck-Condon (FC) calculation was combined with a least-squares curve-fitting analysis. Unlike the case of diatomic molecules, where a onedimensional potential curve determines the vibrational states, in the present case of a polyatomic molecule each normal coordinate has its own set of FC parameters derived from a multidimensional potential surface. The advantage of using normal coordinates is that they can be treated independently in a FC analysis, in the limit of small vibrational quantum numbers. The essential result of this independent-mode approximation is that the relative branching ratios between vibrational levels of a given mode are independent of the quantum numbers of the other modes. For example, the relative intensities of the $(0,0,0),(1,0,0)$, $(2,0,0)$, etc. progression are the same as the relative intensities of the $(0,1,0),(1,1,0)$, $(2,1,0)$, etc. progression, within this approximation.

The multimode FC analysis used here was hased on the single-mode algorithms first given by Hutchisson [10]. In this analysis, only the $v_{1}, v_{2}$, and $v_{3}$ vibrational modes were included. These three modes are expected to dominate the vibrational structure because dipole-allowed electronic excitations will preferentially excite the totally-symmetric a) modes $v_{1}, v_{2}$, and $v_{3}$. Substantial excitation of the $v_{4}\left(b_{1}\right.$ symmetry) and $v_{5}, v_{6}\left(b_{2}\right.$

symmetry) modes are not expected due to selection rules. Since $C_{2 v}$ symmetry is assumed in the final state, the symmetry coordinates associated with the $v_{4}, v_{5}$, and $v_{6}$ modes are not adjusted in the analysis. The possiblity of small contributions to the spectrum from 
excitations of these modes, however, cannot be excluded. Note that the above arguments are only valid for a planar $\mathrm{H}_{2} \mathrm{CO}$ molecule with $\mathrm{C}_{2 v}$ symmetry. The geometry of the (C

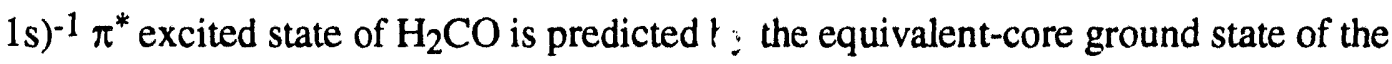
nitroxyl radical $\mathrm{H}_{2} \mathrm{NO}$, which is probably not planar, but has an inversion barrier of only $\approx 6 \mathrm{meV}$ [11]. Since this barrier is much less than the zero-point energy expected for the out-of-plane bending mode, $v_{4}$, the assumption of a planar $\mathrm{H}_{2} \mathrm{CO}$ molecule in the (C 1s) ${ }^{-1}$ $\pi^{*}$ state seems to be reasonable. Note that this slightly non-planar geometry is caused by the unpaired $\pi^{*}$ electron (which actually has $b_{2}$ symmetry). The $a_{1}$-symmetry carbon $1 \mathrm{~s}$ core hole cannot cause deviations from planarity. Thus deviations from planitity should be smaller for the Rydberg states than for the $\pi^{*}$ state, simply because the Rydberg orbitals have less amplitude near the nuclei and the valence electrons. Therefore a planar geometry is also assumed for all the Rydberg states.

As discussed in Section IV.C.2, the contribution of the symmetry coordinates to the normal modes has been calculated for the ground states of $\mathrm{H}_{2} \mathrm{CO}$ and $\mathrm{D}_{2} \mathrm{CO}$. The results are shown in Table IV-2. Note that this transformation matrix is block-diagonalized, e.g. the three $\mathrm{a}_{1}$ symmetry coordinates contribute only to the three $\mathrm{a}_{1}$ normal modes. The changes in normal coordinates upon excitation, derived from the multimode FC analysis, were transformed to symmetry coordinates using this matrix. This assumes that the same transformation matrix applies in the (C 1s) $)^{-1}$ excited states as in the ground state. Because this transformation is derived from the ground-state force constants and geometry of the molecule, this assumption is not strictly valid. Indeed, the observed vibrational excitations and changes in vibrational spacings relative to the ground state indicate that the force constants and geometry do change upon excitation. However, this parallel-mode approximation allows a straightforward interpretation of the results of the FC analysis. The transformation matrix is not expected to change drastically upon core-excitation, so the qualitative results of the analysis are reliable. Because of this approximation, the derived geometric parameters may be subject to somewhat larger errors than the indicated 
uncertainties, which were are based only on the statistical quality of the data and the fit. In fact, some of the results discussed below are more easily understood as artifacts of the parallel-mode approximation than as real structural effects.

As a result of the least-squares analysis, the FC parameters for the (C $1 \mathrm{~s})^{-1} \pi^{*}$ state of $\mathrm{H}_{2} \mathrm{CO}$ were derived, i.e. the vibrational energies $h v_{i}(i=1,2,3)$ and the changes in bond lengths ( $\mathrm{C}-\mathrm{H}$ and $\mathrm{C}-\mathrm{O}$ equilibrium distances $\mathrm{R})$ and in the $\mathrm{HCH}$ molecular bond angle $\theta$, along with the corresponding values for $\mathrm{D}_{2} \mathrm{CO}$. In the $\mathrm{FC}$ calculations, anharmonic Morse potentials were used for these three excited-state vibrational modes, while harmonic potentials were assumed for the ground-state modes, because those anharmonicities are not known. Note that the Morse potential is valid for the $v_{3}$ mode, where contributions from bond-stretching motions will contribute anharmonicity to the potential, even if the H-C-H bending contribution is assumed to be harmonic. The results of the FC analysis are summarized in Table IV-3. The use of the Morse potential in the excited state allows the determination of the sign of the change in symmetry coordinates, i.e. it indicates whether a bond distance increases or decreases.

For the (C 1s) $)^{-1}$ Rydberg states, the lower signal intensities and the overlaps between different Rydberg states make a full FC analysis difficult. The spectra were therefore analyzed by accounting only for two of the vibrational modes $\left(v_{1}, v_{2}\right)$, since the results for the (C $1 s)^{-1} \pi^{*}$ resonance show that the $v_{3}$ mode is only weakly excited. Thus only the vibrational energies $h v_{i}(i=1,2)$ and the changes in bond length were determined for these states (see Table IV-3). Different intermolecular distances are obtained by assuming different $\mathrm{HCH}$ (DCD) molecular bond angles, $\theta$. The values shown in Table IV3 are derived by assuming no change in this angle upon excitation. The derived values change by less than $0.01 \AA$ if the $H C H$ (DCD) angle $\theta$ is varied by $\pm 10^{\circ}$. For the $(C 1 \mathrm{~s})^{-1}$ Rydberg states, a harmonic potential was assumed; thus it is not possible to determine whether an electronic transition takes place to the attractive or the repulsive side of the excited-state potential curve. As a result, the sign of the bond length change is not known. 
Table IV-3 therefore lists two possible equilibrium internuclear distances for each R of the core-excited Rydberg states. One of the values is less likely (see below) and is set in square brackets.

The results show that the equilibrium $\mathrm{C}-\mathrm{O}$ distance in formaldehyde, $\mathrm{R}_{\mathrm{C}-\mathrm{O}}$, increases in the (C 1s) $)^{-1} \pi^{*}$ state relative to the ground state, as also seen for the $\mathrm{CO}$ molecule [7]. In $\mathrm{H}_{2} \mathrm{CO}$, however, this increase $(\approx 0.10 \AA)$ is much larger than in the $\mathrm{CO}$ molecule $(\approx 0.025 \AA)$, so that the intensities of vibrationally excited states with C-O stretching-mode character in the (C $1 \mathrm{~s})^{-1} \pi^{*}$ resonance of formaldehyde are much higher than those of the analogous excitations of $\mathrm{CO}$. While in the (C 1s)-1 $\pi^{*}$ of $\mathrm{CO}$, the intensity of the $v^{\prime}=1$ excitation amounts to only $\approx 10 \%$ of that of the $v^{\prime}=0$ excitation, for formaldehyde the corresponding $v_{2}$ ' excitations are found to be roughly equal in intensity (see solid subspectrum in Fig. IV-2). This is quite similar to the well-known case of the

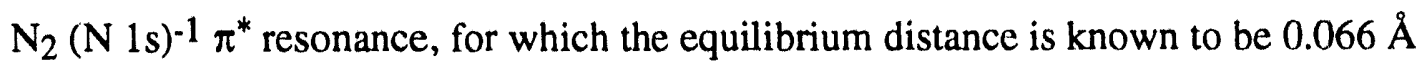
larger than in the ground state [3]. For the $v_{1}$ mode of $\mathrm{H}_{2} \mathrm{CO}$ (mainly symmetric $\mathrm{C}-\mathrm{H}$ stretch), excitation into the lowest $(\mathrm{C} 1 s)^{-1} \pi^{*}$ vibrational state $\left(v_{1}^{\prime}=0\right)$ is strongest (see the relative intensities of the solid, dashed, and dash-dotted subspectra in Fig. IV-2).

For the (C 1s $)^{-1} 3 p-b_{2}$ and $(C 1 s)^{-1} 3 p-b_{2}$ Rydberg states, excitations of the C-O stretching mode $v_{2}$ are much less intense than for the (C 1s $)^{-1} \pi^{*}$ resonance. Similarly, the excitation of the $v_{1}$ vibrational mode is also less intense in the (C 1s) $)^{-1} 3 p$ Rydberg states, as compared to the (C $1 \mathrm{~s})^{-1} \pi^{*}$ resonance. The changes in internuclear equilibrium distances, derived from the FC analysis, are summarized in Table IV-3. While the qualitative trends of the results are believed to be reliable, limitations on the validity of the parallel-mode approximation may introduce inaccuracies in the bond distances which are larger than the statistical error bars shown. For the $(C 1 s)^{-1} \pi^{*}$ resonance, there is a decrease of the $\mathrm{C}-\mathrm{H}(\mathrm{C}-\mathrm{D})$ distance and an increase of the C-O distance with respect to the ground states. This means that the C-H (C-D) bond is strengthened, while the C-O bond is weakened by the excitation. These changes are cunfirmed by the observed increase in the 
$v_{1}$ mode vibrational energy and decrease in the $v_{2}$ mode vibrational energy (see Table IV-

3). Qualitative predictions of these changes can be made by examining the orbital diagrams of Fig. IV-4 (from Ref. [12]). The observed weakening of the C-O bond is expected from the spatial distribution of the antibonding $\pi^{*}\left(b_{2}\right)$ orbital, which is located mainly on $C$ and $\mathrm{O}$, but not on the $\mathrm{H}$ atoms. The influence of the carbon 1s core hole leads to the strengthening of the $\mathrm{C}-\mathrm{H}$ bond because the $\mathrm{C}-\mathrm{H}$ bonding $1 \mathrm{~b}_{1}$ and $4 \mathrm{a}_{1}$ orbitals are somewhat polarized towards the $\mathrm{H}$ atoms. The increased positive charge on the carbon atom pulls these bonding electrons towards the carbon, strengthening the $\mathrm{C}-\mathrm{H}$ bond. As discussed for the $\mathrm{CO}$ molecule [7], the $\mathrm{C}-\mathrm{O}$ bonding orbitals in formaldehyde (in particular, $3 a_{1}$ ) are polarized towards the oxygen atom, so that the carbon core hole will also tend to strengthen the $\mathrm{C}-\mathrm{O}$ bond. For the $(\mathrm{C} 1 \mathrm{~s})^{-1} \pi^{*}$ resonance, this $\mathrm{C}-\mathrm{O}$ strengthening is more than compensated by the $\mathrm{C}-\mathrm{O}$ antibonding character of the $\pi^{*}$ orbital, even more than for the CO molecule [7].

For the (C 1s) $)^{-1}$ Rydberg excitations, the FC analysis of the present data does not indicate whether the bond lengths increase or decrease relative to the ground state. The Rydberg orbitals are expected to be non-bonding, however. Thus, from the arguments given just above, it can be predicted that the carbon 1s core hole will induce a slight decrease of both the $\mathrm{C}-\mathrm{O}$ and $\mathrm{C}-\mathrm{H}$ (C-D) bond lengths. Therefore the smaller value given for each bond length in a (C 1s)-1 Rydberg state in Table IV-3 is preferred; the larger values are considered less likely and are therefore set in square brackets. In either set of values, the magnitude of the change in the $\mathrm{C}-\mathrm{O}$ bond distance is much larger for the (C $1 \mathrm{~s})^{-1} \pi^{*}$ state than for the (C 1s) $)^{-1}$ Rydberg states. This is consistent with the strongly C-O antibonding nature of the $\pi^{*}$ orbital and the essentially nonbonding character of the Rydberg orbitals. The expected strengthening of the C-H (C-D) and C-O bonds in the Rydberg states should be reflected by a corresponding increase in the $v_{1}$ and $v_{2}$ vibrational spacings, as compared to the ground state. Table IV-3 indicates, however, that the vibrational spacings actually decrease in several cases. This can arise, even if the 
characteristic vibrational frequencies of both the $\mathrm{C}-\mathrm{H}(\mathrm{C}-\mathrm{D})$ and $\mathrm{C}-\mathrm{O}$ bonds increase, if the composition of the normal mode changes upon excitation. For example, the observed decrease in the $v_{2}$ vibrational spacing of $\mathrm{H}_{2} \mathrm{CO}$ upon excitation of the $(\mathrm{C} 1 \mathrm{~s})^{-1} 3 \mathrm{p}-\mathrm{b}_{2}$ state may result from larger contributions from the symmetric $\mathrm{C}-\mathrm{H}$ stretch to this normal mode, rather than from a weakening of the $\mathrm{C}-\mathrm{O}$ bond. If these proposed changes in the normalmode compositions actually occur, then the parallel-mode approximation used here is not completely valid.

The derived ( $\mathrm{C} 1 \mathrm{~s})^{-1} \pi^{*}$ molecular geometry of $\mathrm{H}_{2} \mathrm{CO}$ can be compared with calculated values for the ground state of the equivalent-core molecule $\mathrm{H}_{2} \mathrm{NO}[13,14,15]$. The results of these calculations reflect the same trend as found here for the $\mathrm{C}-\mathrm{H}$ and $\mathrm{C}-\mathrm{O}$ distances, but the absolute values $\left(\mathrm{R}_{\mathrm{N}-\mathrm{H}}=0.99 \AA[13,14,15], \mathrm{R}_{\mathrm{N}-\mathrm{O}}=1.30 \AA[13], 1.26 \AA\right.$ [14], and $1.34 \AA$ [15]) are slightly different. A discrepancy is also observed between the calculated $(\mathrm{H} \cdot \mathrm{N}-\mathrm{H})$ molecular bond angle for the ground state of $\mathrm{H}_{2} \mathrm{NO}\left(\theta=116^{\circ}[15]\right)$ and the derived value for $\mathrm{H}_{2} \mathrm{CO}$ in the $(\mathrm{C} 1 \mathrm{~s})^{-1} \pi^{*}$ state.

\section{IV.D. Oxygen K-edge}

Fig. IV-5 shows the oxygen K-edge region of the photoionization spectrum of gasphase formaldehyde. As in the carbon K-edge region, the spectrum is dominated by an intense $(\mathrm{O} 1 \mathrm{~s})^{-1} \pi^{*}$ resonance at $530.8 \mathrm{eV}$ and a series of weaker transitions to $(\mathrm{O} 1 \mathrm{~s})^{-1}$ Rydberg states at higher energies. All these peaks can be assigned in analogy to the corresponding spectrum for gas-phase $\mathrm{CO}$ [7]. The resulting assignments are given in Fig. IV-5 and summarized in Table IV-1, which also shows a comparison with former measurements and theoretical calculations.

The $(\mathrm{O} 1 \mathrm{~s})^{-1} \pi^{*}$ resonance shows a very broad width, presumably due to unresolved vibrational structure, similar to the analogous resonance in $\mathrm{CO}$ [7]. In the present case, however, the $v_{2}$ vibrational spacing (mainly C-O stretch) is apparently below 
the resolution limit [ $\triangle \mathrm{E} \approx 150 \mathrm{meV}$ (FWHM)]. The $\mathrm{v}_{2}$ vibrational spacing for the $(\mathrm{O} 1 \mathrm{~s})^{-1}$ $\pi^{*}$ state can thus be estimated to be $\leq 100 \mathrm{meV}$, smaller than that of the equivalent state in $\mathrm{CO}(\mathrm{hv}=146 \mathrm{meV})$. Such a small $v_{2}$ vibrational spacing is not unreasonable, in view of the present result for the (C $1 \mathrm{~s})^{-1} \pi^{*}$ state, where the $v_{2}$ vibrational spacing was found to be only $\approx 65 \%$ of the corresponding energy in the $\mathrm{CO}$ molecule. Note that, in the ground state, the $\mathrm{H}_{2} \mathrm{CO} v_{2}$ vibrational energy is $\approx 80 \%$ of the $\mathrm{CO}$ vibrational energy. Together, these results suggest that the $\pi^{*}$ orbital is more strongly antibonding (for $\mathrm{C}-\mathrm{O}$ ) in $\mathrm{H}_{2} \mathrm{CO}$ than in CO. This conclusion is also supported by the much larger increase in $\mathrm{C}-\mathrm{O}$ bond length upon excitation of the (C $1 \mathrm{~s})^{-1} \pi^{*}$ state of $\mathrm{H}_{2} \mathrm{CO}$ than for the corresponding excitation in CO. Comparison of the $v_{2}$ vibrational spacing $(160 \mathrm{meV})$ in the (C $\left.1 \mathrm{~s}\right)^{-1} \pi^{*}$ resonance of $\mathrm{H}_{2} \mathrm{CO}$ to the upper limit of $100 \mathrm{meV}$ for the $(\mathrm{O} 1 \mathrm{~s})^{-1} \pi^{*}$ resonance indicates a weaker $\mathrm{C}-\mathrm{O}$ bond in the latter state. This is expected due to the $\mathrm{C}-\mathrm{O}$ bond-weakening influence of the oxygen 1s core-hole.

The energy spacing of the $v_{1}$ vibrational mode is not expected to be less than 100 $\mathrm{meV}$ in the $(\mathrm{O} 1 \mathrm{~s})^{-1} \pi^{*}$ state of formaldehyde, considering the ground-state value of 345.0 $\mathrm{meV}(254.9 \mathrm{meV})$ in $\mathrm{H}_{2} \mathrm{CO}\left(\mathrm{D}_{2} \mathrm{CO}\right)$, and similar values in the $(\mathrm{C} 1 \mathrm{~s})^{-1}$ states (see Table IV3). This suggests that there is no significant vibrational excitation of the $v_{1}$ mode when the oxygen $1 \mathrm{~s}$ electron is excited into the $\pi^{*}$ orbital. Since, on the other hand, strong $v_{1}$ excitations are observed for the (C 1s) ${ }^{-1} \pi^{*}$ state (see Fig. IV-2), it appears that these excitations are caused mainly by the creation of a carbon $1 \mathrm{~s}$ core hole and much less by the addition of an electron to the $\pi^{*}$ molecular orbital. This result supports the interpretations given in the previous section.

The equivalent-core molecule to $\mathrm{H}_{2} \mathrm{CO}$ in the $(\mathrm{O} 1 \mathrm{~s})^{-1} \pi^{*}$ state is ground-state $\mathrm{H}_{2} \mathrm{CF}$. Semi-empirical calculations predict the bond lengths $\mathrm{R}_{\mathrm{C}-\mathrm{H}}=1.11 \AA$ and $\mathrm{R}_{\mathrm{C}-\mathrm{F}}=$ $1.32 \AA$ in that molecule [16]. A comparison of these results to the ground-state values for $\mathrm{H}_{2} \mathrm{CO}$, given in Table IV-3, supports the qualitative arguments given above. The $\mathrm{C}-\mathrm{H}$ bond length is essentially unchanged, while the $\mathrm{C}-\mathrm{O}(\mathrm{C}-\mathrm{F})$ bond length shows a large 
increase going from $\mathrm{H}_{2} \mathrm{CO}$ to $\mathrm{H}_{2} \mathrm{CF}$.

The lowest $(\mathrm{O} 1 \mathrm{~s})^{-1}$ Rydberg state is assigned to a transition to the $(\mathrm{O} 1 \mathrm{~s})^{-1} 3 \mathrm{~s}-\mathrm{a}_{1}$ state. This peak is quite broad, as seen above for the $(\mathrm{C} 1 \mathrm{~s})^{-1} 3 \mathrm{~s}-\mathrm{a}_{1}$ transition. The remaining (O 1s) $)^{-1}$ Rydberg peaks arise from transitions into np orbitals (up to $n=7$ ) and converge to the oxygen 1s ionization threshold IPO-K. A least-squares fit of this series with the Rydberg formula gives $I_{\mathrm{O}} \mathrm{K}=539.30 \mathrm{eV}$ and $\delta_{\mathrm{p}}=1.01$. This assignment is much more consistent than that given in Ref. [1], where the most intense peak was assigned to the $(\mathrm{O} 1 \mathrm{~s})^{-1} 4 \mathrm{~s}$ state. All the Rydberg states are noticeably asymmetric, with tails extending to higher energies. This is probably due to unresolved vibrational sidebands of the $v_{2}$ mode, with vibrational energies apparently smaller than those in the corresponding states of $\mathrm{CO}(\mathrm{h} v \approx 225 \mathrm{meV})[7]$. As seen for the $(\mathrm{O} 1 \mathrm{~s})^{-1} \pi^{*}$ resonance, the $v_{1}$ vibrational mode does not seem to be noticeably excited in the $(\mathrm{O} 1 \mathrm{~s})^{-1}$ Rydberg states. Apparently the oxygen 1s core hole has little influence on the strength of the $\mathrm{C}-\mathrm{H}$ bonds.

The lower spectrum in Fig. IV-5 is the (O 1s) $)^{-1}$ photoionization spectrum of $\mathrm{D}_{2} \mathrm{CO}$. Clearly there are only minor isotopic differences observed in this energy range. This is mainly because vibrational fine structures are not resolved in these $(\mathrm{O} 1 \mathrm{~s})^{-1}$ core-excitation spectra, giving rise, at best, to high-energy shoulders of the observed peaks. A notable isotopic effect is observed for the $(\mathrm{O} 1 \mathrm{~s})^{-1} 3 \mathrm{~s}-\mathrm{a}_{1}$ Rydberg state; there is a decrease of linewidth and a shift to higher energies upon deuteration. These effects are discussed below along with the very similar observations for the $(\mathrm{C} 1 \mathrm{~s})^{-1} 3 \mathrm{~s}-\mathrm{a}_{1}$ state.

\section{IV.E. Isotopic effects}

\section{Isotopic effects on vibrational fine structure}

It has already been mentioned above that all observed vibrational modes in these spectra exhibit isotopic effects, even though only $\mathrm{H}$ (and not $\mathrm{C}$ or $\mathrm{O}$ ) was replaced by the 
heavier isotope (D). This applies, in particular, to the $v_{2}$ mode, which consists mainly of symmetric $\mathrm{C}-\mathrm{O}$ stretching motions. To understand these isotopic effects, one must consider the contributions from all the different symmetry coordinates to the various normal modes. These contributions were determined for the ground electronic states of $\mathrm{H}_{2} \mathrm{CO}$ and $\mathrm{D}_{2} \mathrm{CO}$ by a normal-mode analysis, as described above, with the results shown in Table IV2. Note that the contributions of the symmetry coordinates to a given normal vibrational mode change strongly upon deuteration, e.g. whereas the $\mathrm{v}_{1}$ mode in $\mathrm{H}_{2} \mathrm{CO}$ consists of $\approx 78 \% \mathrm{C}$-H symmetric stretch, only $\approx 50 \%$ of C-D symmetric stretch contributes to this mode in $\mathrm{D}_{2} \mathrm{CO}$. For the $\mathrm{v}_{2}$ mode, the contribution of the symmetric C-H (C-D) stretching vibration increases from $\approx 10 \%$ in $\mathrm{H}_{2} \mathrm{CO}$ to $\approx 26 \%$ in $\mathrm{D}_{2} \mathrm{CO}$.

The observed effects of deuteration on the vibrational energies in the core-excited states can only be discussed in a qualitative way, since the relative contributions of the symmetry coordinates to the normal modes are not known for these states. It is generally expected, however, that all the vibrational energies will decrease upon deuteration, if the composition of the normal modes does not change. The results of Table IV-3 show that decrease is indeed observed for the (C $1 \mathrm{~s})^{-1}$ states. The only exception is in the (C $\left.1 \mathrm{~s}\right)^{-1} \pi^{*}$ resonance, where $\mathrm{hv}_{2}$ increases by $12 \pm 6 \%$ upon deuteration. An increase in vibrational energy is possible, despite decreasing energies of all the symmetry-coordinate vibrational energies, if the contribution to this mode from the $\mathrm{C}-\mathrm{H}$ symmetric-stretch coordinate, with its high vibrational energy, increases upon deuteration. Comparing the compositions of the $v_{2}$ normal mode in the ground states of the two isotopic species, as shown in Table IV-2, it is seen that the high-frequency $\mathrm{C}-\mathrm{H}(\mathrm{C}-\mathrm{D})$ stretch makes a significantly larger contribution in $\mathrm{D}_{2} \mathrm{CO}$ than in $\mathrm{H}_{2} \mathrm{CO}$. This explains the very small decrease observed for the groundstate vibrational energy hv 2 upon deuteration, as shown in Table IV-3. Apparently in the (C $1 \mathrm{~s})^{-1} \pi^{*}$ excited state, the compositions of the normal modes have changed enough, relative to the ground state, to make $h v_{2}$ increase upon deuteration. This is a direct demonstration that the parallel-mode approximation has limited validity for the (C $1 \mathrm{~s})^{-1} \pi^{*}$ 
excited state.

The changes in bond distances, going from $\mathrm{H}_{2} \mathrm{CO}$ to $\mathrm{D}_{2} \mathrm{CO}$, which are small in the molecular ground state $[-0.004$ and $-0.0005 \AA$ for the $\mathrm{C}-\mathrm{H}(\mathrm{D})$ and $\mathrm{C}-\mathrm{O}$ distances, respectively], are much larger in the (C $1 \mathrm{~s})^{-1}$ core-excited states. In the (C $\left.1 \mathrm{~s}\right)^{-1} \pi^{*}$ state, for example, they amount to $+0.054 \pm 0.008$ and $-0.014 \pm 0.010 \AA$ for the C-H(D) and C-O distances, respectively. Thus, for the (C 1s) $)^{-1} \pi^{*}$ state, the C.D distance in $\mathrm{D}_{2} \mathrm{CO}$ is larger than the $\mathrm{C}$ - $\mathrm{H}$ distance in $\mathrm{H}_{2} \mathrm{CO}$, while the opposite is observed for the $\mathrm{C}$-O distance. This is in qualitative agreement with the observed decrease (or increase) in vibrational frequency of the $v_{1}$ (or $v_{2}$ ) excited-state mode upon deuteration. Of course, the bond lengths for the (C 1s) $)^{-1}$ states were derived using the parallel-mode approximation, i.e. by assuming the composition of the normal modes is unchanged upon excitation. The actual compositions of the normal modes in these states, which are not known, might lead to derived geometries for $\mathrm{H}_{2} \mathrm{CO}$ and $\mathrm{D}_{2} \mathrm{CO}$ with smaller isotopic differences than are obtained here. In fact, that situation seems more likely than the rather large isotopic effects on molecular geometry derived in the present analysis.

\section{Isotopic effects on the (C 1s) $)^{-1} 3 s-a_{1}$ and $\left(\begin{array}{ll}O & 1 s\end{array}\right)^{-1} 3 s-a_{1}$ Rydberg states}

Table IV-4 summarizes the results for the linewidths, W, and the energy positions, $\mathrm{E}$, of the $(\mathrm{C} 1 \mathrm{~s})^{-1} 3 \mathrm{~s}-\mathrm{a}_{1}$ and $(\mathrm{O} 1 \mathrm{~s})^{-1} 3 \mathrm{~s}-\mathrm{a}_{1}$ Rydberg states as obtained from the leastsquares analysis. For both of these Rydberg states, a substantial decrease in linewidth and a shift of the excitation energy to higher values are observed when $H$ is replaced by $D$. The isotopic effects appear to be larger for the $(C 1 \mathrm{~s})^{-1} 3 \mathrm{~s}-\mathrm{a}_{1}$ state $(\Delta \mathrm{W}=-60 \mathrm{meV}, \Delta \mathrm{E}=+54$ $\mathrm{meV})$ than for the $(\mathrm{O} 1 \mathrm{~s})^{-1} 3 \mathrm{~s}-\mathrm{a}_{1}$ state $(\Delta \mathrm{W}=-44 \mathrm{meV}, \Delta \mathrm{E}=+40 \mathrm{meV})$.

There are three unusual effects observed for the $(C 1 s)^{-1} 3 s-a_{1}$ and $(O 1 s)^{-1} 3 s-a_{1}$ Rydberg states: (1) The fundamental transition shifts to higher energy upon deuteration; (2) The linewidth decreases upon deuteration; (3) The linewidth is larger than the 
linewidths of other Rydberg states (with the same core hole). Effect (1) may be explained by a large change in the vibrational energies of the molecule upon excitation, which implies a different zero-point vibrational energy contribution in the initial and final states, with an accompanying isotopic dependence of the fundamental transition energy. These zero-point influences on the energies of fundamental transitions were discussed in Section I.D.6. The contribution of vibrational mode $i$ to the shift of the fundamental transition energy upon deuteration is given by $\Delta \mathrm{E}_{1}=(1 / 2)\left[h v_{i}{ }^{\prime \prime}\left(\mathrm{H}_{2} \mathrm{CO}\right)-h v_{1}^{\prime \prime}\left(\mathrm{D}_{2} \mathrm{CO}\right)-h v_{j}^{\prime}\left(\mathrm{H}_{2} \mathrm{CO}\right)+h v_{1}^{\prime}\left(\mathrm{D}_{2} \mathrm{CO}\right)\right]$. Of course, the largest changes in vibrational energy generally occur when the final state has a purely repulsive potential for one (or more) of the vibrational modes, i.e. the final-state vibrational energy of that mode is zero. In that case, the resulting dissociation of the molecule can occur so quickly that it will compete with the autoionization decay of the excited state, leading to a larger natural linewidth. This rapid neutral dissociation has been observed for core-excited valence-shell states in $\mathrm{HBr}$ [17], $\mathrm{HCl}$ [18], and $\mathrm{H}_{2} \mathrm{~S}$ [19]. This process would explain effect (3), with the dissociation presumably involving removal of one of the $H(D)$ atoms.

If the dissociation rate is smaller for $\mathrm{D}_{2} \mathrm{CO}$ than for $\mathrm{H}_{2} \mathrm{CO}$, this model also explains effect (2). This isotopic dependence of the rate can arise if there is a small barrier to dissociation along the excited-state potential, such that the $\mathrm{H}$ atom can rapidly tunnel through the baurier, whereas the $D$ atom tunnels more slowly because of its larger mass. Of course, if there is such a barrier to dissociation, there is a zero-point vibrational energy for the associated vibrational mode. While this implies a smaller isotopic effect on vibrational zero-point energy than for a purely repulsive potential, the excited-state vibrational energy of the dissociative mode could be significantly smaller than in the initial state, contributing to the observed isotopic shift of the fundamental transition. To summarize, effect (1) is explained by any large changes in the vibrational energies upon excitation, including those arising from a purely repulsive excited-state potential. Effect (2) suggests a dissociative decay of the neutral excited state, which proceeds faster than 
autoionization. And effect (3) can arise if the dissociation potential includes a barrier which is penetrated faster by a $H$ atom than by a $D$ atom.

This interpretation of the observed effects suggests that the core-excited 3s-a 1 state are dissociative within the lifetime of the core hole. Resonant Auger electron spectroscopy and resonant photo-ion mass spectroscopy could be used to study these states, and possibly determine if this model is correct. Those techniques, along with theoretical calculations, were used to characterize the dissociative states of $\mathrm{H}_{2} \mathrm{~S}$ below the sulfur $2 p$ ionization threshold [19]. This possibility indicates the ability of high-resolution XANES to identify promising directions for more detailed investigations of core-excited electronic states.

For the $(C / s)^{-1} 3 s-a_{1}$ state, the $v_{1}$ and $v_{2}$ vibrational energies are known from the leas ' squares analysis [hv ${ }^{\prime}=324(236) \mathrm{meV}, h_{2}^{\prime}=123(106) \mathrm{meV}^{\prime}$ for $\mathrm{H}_{2} \mathrm{CO}\left(\mathrm{D}_{2} \mathrm{CO}\right)$ ]. The combined contribution of these two modes to the isotopic shift of the fundamental transition energy is therefore $\Delta E_{1,2}=-5 \mathrm{meV}$. This implies that $+59 \mathrm{meV}$ of the observed shift is caused by the other four modes. If the excited state is dissociative, with a purely repulsive potential surface that rapidly expels a $H(D)$ atom, then the $v_{5}$ vibrational mode [predominantly asymmetric C-H (C-D) stretch] is essentially absent in the excited state. This creates an isotopic shift $\Delta E_{9}=+42 \mathrm{meV}$, using the ground state vibrational energies from ref. [6]. In this case, the combined shift from the $v_{1}, v_{2}$, and $v_{5}$ modes, $\Delta E_{1,2.5}=+37 \mathrm{meV}$, is somewhat smaller than the observed shift of $+54 \mathrm{meV}$; the difference can be attributed to changes in the other three vibrational energies and/or by the uncertainties of the measulements.

A further consideration in the interpretation of the observed isotopic energy shifts is the relative energy calibration of the $\mathrm{H}_{2} \mathrm{CO}$ and $\mathrm{D}_{2} \mathrm{CO}$ spectra. The present results indicate that the spectra of the two isotopic species compared in Figs. IV-2, IV-3, and IV-5 are aligned, i.e. the fundamental transitions (except those the 3s-al orbital) show no energy shift upon deuteration. However, due to difficulties in the absolute calibration of energy in 
these measurements, there is possibly some error in the relative alignment of the energy scales for $\mathrm{H}_{2} \mathrm{CO}$ and $\mathrm{D}_{2} \mathrm{CO}$. Such a shift would appear if the zero-point vibrational energy of the core-ionized molecule, e.g. $\mathrm{H}_{2} \mathrm{C}^{*} \mathrm{O}^{+}$, is different from that of the ground state. This difference would be observed as a uniform isotopic energy shift of the upper Rydberg states, caused by a corresponding shift in the ionization threshold. Therefore such a shift is expected if the core-ionized molecule has significant changes in vibrational energies, relative to the ground state. In case of carbon Is ionization, it was predicted above that the carbon core hole will tend to strengthen the bonding. This should be reflected by larger vibrational energies, if the composition of the normal modes is not modified too much by the excitation. This would result in an shift of the upper Rydberg states, and the carbon $1 \mathrm{~s}$ ionization threshold, to lower energies upon deuteration. While the observed shift of the (C $1 \mathrm{~s})^{-1} 3 \mathrm{~s}-a_{1}$ state upon deuteration would he increased hy this difference in ionization threshold energy, the part of that shift due to the unique character of the $3 \mathrm{~s}$-al orbital would still be the value reported here. It would be desirable, in regard to these issues, to measure the carbon $1 \mathrm{~s}$ binding energies of $\mathrm{H}_{2} \mathrm{CO}$ and $\mathrm{D}_{2} \mathrm{CO}$ by $\mathrm{x}$-ray photoelectron spectroscopy with high resolution, and thereby independently determine whether an isotopic shift of the threshold exists.

\section{Comparison to the $\left(2 b_{1}\right)^{-1}$ valence-excited Rydberg states of formaldehyde}

The preceding section shows that the assumption of a rapidly dissociating $(\mathrm{C} 1 \mathrm{~s})^{-1}$ 3s-al state provides a plausible explanation for all three observed effects, and in particular gives a reasonable prediction of the observed isotopic energy shift. Presumably a similar explanation could apply for the $(\mathrm{O} 1 \mathrm{~s})^{-1} 3 \mathrm{~s}-\mathrm{a}_{1}$ state, since the difference in the location of the core hole should not affect the dissociative nature of the state. It is somewhat unexpected, however, that promoting an electron to a Rydberg orbital creates a dissociative 
potential. In fact, the characterization of the $3 \mathrm{~s}$ orbital in these states purely as a Rydberg orbital is probably inaccurate, based upon the observed behavior. This orbital may have intermediate character, i.e. it may resemble a valence orbital to some extent. This possibility was already mentioned by Lessard and Moule for the $3 \mathrm{~s}$ orbital in the $\left(2 \mathrm{~b}_{1}\right)^{-1} 3 \mathrm{~s}$ a) valence-excited state of formaldehyde [20]. In that case, they suggest that the intermediate character of the $3 \mathrm{~s}$ orbital may arise from mixing of the $\left(2 b_{1}\right)^{-1} 3 \mathrm{~s}-\mathrm{a}_{1}$ state with the $\left(2 b_{1}\right)^{-1} \sigma^{*}$ inter-valence state at $\boldsymbol{m} 3.5 \mathrm{eV}$ higher energy (see Section 1.D.3). Such mixing seems less likely for these core-excited states, because the corresponding (C $1 \mathrm{~s})^{-1}$ $\sigma^{*}$ resonance is observed well above threshold, $-19 \mathrm{eV}$ higher than the $(C \mathrm{ls})^{-1} 3 \mathrm{~s}$-a transition (see Fig. IV-1). In any case, the sign of the observed isotopic energy shift indicates that the overall bonding is weaker in the $(\mathrm{C} 1 \mathrm{~s})^{-1} 3 \mathrm{~s}-\mathrm{a}_{1}$ and $(\mathrm{O} / \mathrm{s})^{-1} 3 \mathrm{~s}-\mathrm{a}_{1}$ states than in the ground state, and therefore 3s-a, orbital in these states must be somewhat antibonding.

The study of the valence-excited Rydberg states of formaldehyde by Lessard and Moule [20] is of interest, because they observed notable isotopic shifts of the fundamental energies, which are largest for the $\left(2 b_{1}\right)^{-1} 3 \mathrm{~s}-\mathrm{a}_{1}$ state $(\Delta E=+39 \mathrm{meV})$. They presert a detailed discussion of isotopic energy shifts in electronic transitions, based upon the same zero-point vibrational energy arguments discussed here. Isotopic energy shifts were observed for the $\left(2 b_{1}\right)^{-1} 3 p-a_{1}$ and $\left(2 b_{1}\right)^{-1} 3 p-b_{1}$ states, which were $-60 \%$ of the shifts seen for the $\left(2 b_{1}\right)^{-1} 3 s-a_{1}$ state. The present results do not indicate corresponding isotopic shifts for any of the $(\mathrm{C} 1 \mathrm{~s})^{-1} 3 \mathrm{p}$ or $(\mathrm{O} 1 \mathrm{~s})^{-1} 3 \mathrm{p}$ states.

\section{Similar isotopic effects in the $(C 1 s)^{-1} 3 \mathrm{~s}$ states of other molecules}

A survey of high-resolution XANES spectra shows that the effects seen for the core-excited 3s Rydberg states of formaldehyde are not unique. In fact, an isotopic shift of the fundamental transition energy was observed for the $(\mathrm{C} 1 \mathrm{~s})^{-1} 3 \mathrm{~s}$ states of propane, 
ethane, and possibly methane, when compared to the fully deuterated isotopic species [21]. In particular, the energy of the $(\mathrm{C} 1 \mathrm{~s})^{-1} 3 \mathrm{~s}$ statc increases $=20 \mathrm{meV}$ between $\mathrm{C}_{2} \mathrm{H}_{6}$ and $\mathrm{C}_{2} \mathrm{D}_{6} ;$ and a similar amount hetween $\mathrm{C}_{3} \mathrm{H}_{8}$ and $\mathrm{C}_{3} \mathrm{D}_{8}$. A smaller increase was proposed, but not direculy observed, between $\mathrm{CH}_{4}$ and $\mathrm{CD}_{4}$. No other fundamental transitions in these spectra showed isotopic shifts. In contrast to the present case of formaldehyde, notable isotopic effects upon the (C $1 \mathrm{~s})^{-1} 3 \mathrm{~s}$ linewidths were not observed in the alkane spectra [21]. The carbon K-edge spectra of ethylene exhibit similar isotopic effects, as shown in Ref. [22]. In that case, a comparison of the spectra of $\mathrm{C}_{2} \mathrm{H}_{4}$ and $\mathrm{C}_{2} \mathrm{D}_{4}$, and the derived transition energies, shows that most of the fundamental transitions below the carbon $\mathrm{K}$ edge are shifted $-20 \mathrm{meV}$ upon deuteration. This is indicative of an isotopic shift of the carbon Is ionization threshold, as discussed above in Section IV.E.2. The (C Is $)^{-1}$ 3 resonance, however, shows no shift: by necessity since the two spectra were aligned using this feature. It may be that the spectra are in fact misaligned, i.e. there is a $+20 \mathrm{meV}$ shift of the $(\mathrm{C} 1 \mathrm{~s})^{-1} 3 \mathrm{~s}$ resonance upon deuteration, and the other fundamentals do not shift. In any case, the energy separations between the $(C \text { is })^{-1} 3 \mathrm{~s} \mathrm{state}$ and the higherenergy fundarnental transitions are reduced upon deuteration, as seen for formaldehyde and the alkanes listed above. Ref. [22] also presented spectra of $\mathrm{C}_{2} \mathrm{H}_{2}$ and $\mathrm{C}_{2} \mathrm{D}_{2}$, but those results do not clearly indicate any notable isotopic shifts of the $(C \text { ls })^{-1} 3 \mathrm{~s}$ state.

\section{An alternative explanation for the isotopic effects on the (C $1 \mathrm{~s})^{-1} 3 \mathrm{~s}-\mathrm{a}_{1}$

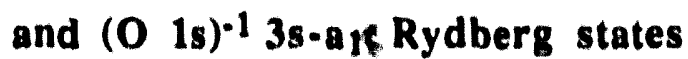

The zero-point vibrational-energy explanation of the isotopic effects, as given above in Section IV.E.2, is very likely correct, insofar as it addresses energy shifts. The only assumption was that fairly large changes in the vibrational energies cccurred upon excitation. However, to explain the large linewidths of the $(\mathrm{C} 1 \mathrm{~s})^{-1} 3 \mathrm{~s}-\mathrm{a}_{1}$ and $(\mathrm{O} 1 \mathrm{~s})^{-1} 3 \mathrm{~s}-$ a1 states, and the isotopic dependence of those widths, it was necessary to assume that 
these states were rapidly dissociating. This behavior is rather surprising, even if the $3 \mathrm{~s}$ Rydberg orbital is presumed to have acquired some valence character. In their analysis of the $\left(2 b_{1}\right)^{-1} 3 s-a_{1}$ state of formaldehyde, Lessard and Moule apparently found no indication of dissociative behavior [20]. The known cases of rapidly dissociating core-excited states clearly involve a transition to a valence-shell orbital, not to an intermediate or Rydberg orbital $[17,18,19]$. In the absence of experimental results to better characterize the (C $1 s)^{-1}$ $3 s-a_{1}$ and $(\mathrm{O} 1 \mathrm{~s})^{-1} 3 \mathrm{~s}-\mathrm{a}_{1}$ states, the following alternative explanation, which does not assume a dissociative state, is worth consideration.

The isotopic effects upon linewidth in the (C 1s $)^{-1} 3 s-a_{1}$ and $(\mathrm{O} 1 \mathrm{~s})^{-1} 3 \mathrm{~s}-\mathrm{a}_{1}$ states may be a result of the large size of the formaldehyde molecule, which causes the deepest Rydberg orbital to be perturbed by the molecular orbitals. As a consequence, this orbital is no longer purely atomic-like; it becomes a mixed orbital. The observed effects suggest that this deviation is more pronounced for $\mathrm{H}_{2} \mathrm{CO}$ (larger linewidth, lower energy) than for $\mathrm{D}_{2} \mathrm{CO}$, which is plausible, if the $\mathrm{D}_{2} \mathrm{CO}$ molecule is assumed to bo smaller in the coreexcited $3 s-a_{1}$ states as compared to $\mathrm{H}_{2} \mathrm{CO}$ in the corresponding states. This is reflected in the FC fits results for the (C $1 s)^{-1} 3 p-b_{2}$ states in the two molecules (see Table IV-3), which indicate that the C-D bond length is shorter by $\approx 0.03 \AA$ as compared to the C-H bond length.

The isotopic effect on the energy of the core-excited $3 s-a_{1}$ state (see Table IV-4) is then explained on the basis of an interaction with the molecular orbitals. In the larger coreexcited molecule, $\mathrm{H}_{2} \mathrm{CO}$, the $3 \mathrm{~s}$ - $\mathrm{a}_{1}$ electron penetrates the screening electrons more and sees a less completely screened nuclear potential. This causes an increase in the quantum defect and a corresponding decrease in the energy of the core-excited state. In $\mathrm{D}_{2} \mathrm{CO}$, the effect is reduced due to the smaller size of the molecule. The magnitude of this energy effect may be comparable to that of the zero-point vibrational-energy effect described above, accounting for some of the observed energy shift. A strong dependence of the energy of the (C $1 \mathrm{~s})^{-1} 3$ s state upon molecular geometry has also been suggested for $\mathrm{CO}_{2}$ 
[23].

The large width of the (C 1s) $)^{-1} 3 s-a_{1}$ state as well as the observed isotopic effect (see Table IV-4) are then explained by a breakdown of the Born-Oppenheimer approximation. For a Rydberg orbital that is perturbed by molecular orbitals, the orbital energy will depend on the size of the molecule, as discussed above. In this case, a coupling between the orbital energy and the zero-point vibrational motion of the groundstate molecule is possible, since the excitation energy may then depend on the size of the molecule at the instant of excitation. The result is a distribution of excitation energies, observed as a non-Lorentzian line broadening. Again the effect will be larger for $\mathrm{H}_{2} \mathrm{CO}$ than for $\mathrm{D}_{2} \mathrm{CO}$ due to molecular size.

It might be expected that these isotopic effects are larger for the $(\mathrm{O} 1 \mathrm{~s})^{-1} 3 \mathrm{~s}-\mathrm{a}_{1}$ state, as compared to the (C 1s)-1 $3 s-a_{1}$ state, because in the former case, the Rydberg orbital is less centered on the molecule. This is not borne out by the results listed in Table IV-4. One reason is that the vibrational fine structure of the $(\mathrm{O} 1 \mathrm{~s})^{-1} 3 \mathrm{~s}-\mathrm{a}_{1}$ state was not resolved, so that the vibrational contributions, which were deconvoluted for the (C 1s $)^{-1} 3 s-a_{1}$ state, could not be removed. This explains the smaller isotopic effect upon the energy of the observed peak; the smaller vibrational spacings in $\mathrm{D}_{2} \mathrm{CO}$, as compared to $\mathrm{H}_{2} \mathrm{CO}$, will lead to an underestimate of the isotopic energy shift of the fundamental transition, if the fine structure is not resolved. 


\section{REFERENCES:}

1. A. P. Hitchcock and C. E. Brion, J. Electron Spectrosc. Relat. Phenom. 19, 231 (1980).

2. J. Schirmer, A. Barth, and F. Tarantelli, Chem. Phys. 122, 9 (1988).

3. C. T. Chen, Y. Ma, and F. Sette, Phys. Rev. A40, 6737 (1989).

4. E. B. Wilson, Jr., J. C. Decius, and P. C. Cross, Molecular Vibrations (Dover, New York, 1955).

5. I. C. Hisatsune and D. F. Eggers, Jr., J. Chem. Phys. 23, 487 (1955).

6. D. J. Cloutier and D. A. Ramsey, Annu. Rev. Phys. Chem. 34, 31 (1983).

7. See Chapter III of this dissertation.

8. D. C. Moule and A. D. Walsh, Chem. Rev. 75, 67 (1975).

9. J. Schirmer (private communication).

10. E. Hutchisson, Phys. Rev. 36, 410 (1930); Phys. Rev. 37, 45 (1931).

11. O. Kysel, P. Mach, and M. Haring, J. Mol. Struct. (THEOCHEM) 138, 299 (1986).

12. W. L. Jorgensen and L. Salem, The Organic Chemist's Book of Orbitals (Academic, New York, 1973) p.84.

13. V. Barone, P. L. Christinziano, F. Lelj, and A. Pastore, J. Mol. Struct. (THEOCHEM) 90, 59 (1982).

14. T. D. Davis, R. E. Christoffersen, and G. M. Maggiora, J. Am. Chem. Soc. 97, 1347 (1975).

15. A. W. Salotti and L. Burnelle, J. Chem. Phys. 53, 333 (1970).

16. M. S. Gordon and J. A. Pople, J. Chem. Phys. 49, 4643 (1968).

17. P. Morin and I. Nenner, Phys. Rev. Lett. 56, 1913 (1986).

18. H. Aksela, S. Aksela, M. Ala-Korpela, O. P. Sairanen, M. Hotokka, G. M. Bancroft, K. H. Tan, and J. Tulkki, Phys. Rev. A 41, 6000 (1990).

19. See the discussion and references in Section VI.F of this dissertation

20. C. R. Lessard and D. C. Moule, J. Chem. Phys. 66, 3908 (1977).

21. G. Remmers, M. Domke, and G. Kaindl, Phys. Rev. A 47, 3085 (1993).

22. Y. Ma, C. T. Chen, G. Meigs, K. Randall, and F. Sette, Phys. Rev. A44, 1848 (1991).

23. W. H. E. Schwarz and R. J. Buenker, Chem. Phys. 13, 153 (1976). 
Table IV-1: Energies (in eV) of the (C $1 \mathrm{~s})^{-1}$ and $(\mathrm{O} 1 \mathrm{~s})^{-1}$ core-excited states of $\mathrm{H}_{2} \mathrm{CO}$ in

\begin{tabular}{cllll}
\hline Assignment & This work & EELS & Theory & Theoryc \\
\hline$(\mathrm{C} 1 \mathrm{~s})^{-1} \pi^{*}$ & 285.590 & 285.7 & 286.06 & 286.02 \\
$3 \mathrm{~s}-\mathrm{a}_{1}$ & 290.178 & 290.15 & 290.64 & 290.50 \\
$3 \mathrm{p}-\mathrm{b}_{2}$ & 291.253 & 291.21 & 291.63 & 291.50 \\
$3 \mathrm{p}-\mathrm{b}_{1}$ & 291.729 & 291.64 & 292.05 & 291.91 \\
$4 \mathrm{~s}-\mathrm{a} 1$ & 292.217 & 292.68 & 293.00 & 292.70 \\
$3 \mathrm{~d}$ & 292.755 & 292.10 & & \\
$4 \mathrm{p}-\mathrm{b}_{2}$ & 292.978 & 292.95 & 293.27 & 293.06 \\
$4 \mathrm{p}-\mathrm{b}_{1}$ & 293.085 & & 293.32 & 293.21 \\
$4 \mathrm{~d}$ & 293.559 & & & \\
$5 \mathrm{p}$ & 293.460 & 293.45 & & \\
$6 \mathrm{p}$ & 293.811 & 293.92 & & \\
$7 \mathrm{p}$ & 293.963 & & & \\
$8 \mathrm{p}$ & 294.057 & & & \\
$9 \mathrm{p}$ & 294.123 & & & \\
$(\mathrm{O} 1 \mathrm{~s})^{-1} \pi^{*}$ & 530.82 & 530.80 & 529.78 & \\
$3 \mathrm{~s}-\mathrm{a}_{1}$ & 535.43 & 535.47 & 534.50 & \\
$3 \mathrm{p}-\mathrm{a}_{1}$ & 536.34 & 536.13 & 535.27 & \\
$4 \mathrm{~s}-\mathrm{a}_{1}$ & 537.78 & 537.65 & 536.54 & \\
$4 \mathrm{p}$ & 538.46 & & 536.69 & \\
$5 \mathrm{p}$ & 538.73 & & & \\
$6 \mathrm{p}$ & 538.94 & & & \\
$7 \mathrm{p}$ & & & \\
\hline \hline
\end{tabular}

comparison with previous EELS results and theoretical predictions. Only fundamental transitions are shown, i.e. transitions including vibrational excitation are not listed.

aRef. [1]

bRef. [2]

cRef. [9] 
Table IV-2: Contributions, in percent, of the symmetry coordinates to the normal modes in the ground states of $\mathrm{H}_{2} \mathrm{CO}$ and $\mathrm{D}_{2} \mathrm{CO} . \mathrm{v}_{4}$ is assumed to consist of $100 \%$ out-of-plane bending motions (see Ref. [5]); it is therefore omitted from the table.

\begin{tabular}{|c|c|c|c|c|c|}
\hline $\mathrm{H}_{2} \mathrm{CO}$ & $v_{1}$ & $v_{2}$ & $v_{3}$ & $v_{5}$ & $v_{6}$ \\
\hline $\begin{array}{l}\mathrm{C}-\mathrm{H} \text { symmetric stretch }\left(\mathrm{a}_{1}\right) \\
\mathrm{C}-\mathrm{O} \text { symmetric stretch }\left(\mathrm{a}_{1}\right) \\
\mathrm{HCH} \text { scissors bend }\left(\mathrm{a}_{1}\right) \\
\mathrm{C}-\mathrm{H} \text { antisymmetric stretch }\left(\mathrm{b}_{2}\right) \\
\mathrm{CH} \mathrm{H}_{2} \text { in-plane wag }\left(\mathrm{b}_{2}\right)\end{array}$ & $\begin{array}{l}77.6 \\
21.1 \\
1.3 \\
0 \\
0\end{array}$ & $\begin{array}{l}9.9 \\
82.3 \\
7.8 \\
0 \\
0\end{array}$ & $\begin{array}{c}2.0 \\
74.1 \\
23.9 \\
0 \\
0\end{array}$ & $\begin{array}{c}0 \\
0 \\
0 \\
96.8 \\
3.2\end{array}$ & $\begin{array}{c}0 \\
0 \\
0 \\
19.2 \\
80.8\end{array}$ \\
\hline \multicolumn{6}{|l|}{$\mathrm{D}_{2} \mathrm{CO}$} \\
\hline $\begin{array}{l}\text { C-D symmetric stretch }\left(a_{1}\right) \\
\text { C-O symmetric stretch }\left(a_{1}\right) \\
\text { DCD scissors bend }\left(a_{1}\right) \\
C-D \text { antisymmetric stretch }\left(b_{2}\right) \\
C D_{2} \text { in-plane wag }\left(b_{2}\right)\end{array}$ & $\begin{array}{l}50.3 \\
47.4 \\
2.3 \\
0 \\
0\end{array}$ & $\begin{array}{l}26.1 \\
71.3 \\
2.6 \\
0 \\
0\end{array}$ & $\begin{array}{r}3.0 \\
59.9 \\
37.1 \\
0 \\
0\end{array}$ & $\begin{array}{r}0 \\
0 \\
0 \\
94.1 \\
5.9\end{array}$ & $\begin{array}{c}0 \\
0 \\
0 \\
30.6 \\
69.4\end{array}$ \\
\hline
\end{tabular}


Table IV-3: Derived equilibrium values for the C-O and C.H (C-D) bond lengths, $R$ (in angstroms), and the $\mathrm{HCH}(\mathrm{DCD})$ molecular bond angle $\theta$, and vibrational energies $\mathrm{h} v$ (in $\mathrm{meV}$ ) for the $v_{1}, v_{2}$, and $v_{3}$ modes in the ground and core-excited states of $\mathrm{H}_{2} \mathrm{CO}$ and $\mathrm{D}_{2} \mathrm{CO}$. The ground-state values were taken from Ref. [6]. The parameters for core-excited states were obtained from least-squares Franck-Condon fits. The numbers in parenthesis give the statistical error bars in units of the last digit. Systematic errors arising from the limited validity of the parallel-mode approximation may be larger. The numbers in square brackets are those results from FC fits which are considered less likely on the basis of chemical arguments (see text).

\begin{tabular}{|c|c|c|c|c|c|c|}
\hline $\mathrm{H}_{2} \mathrm{CO}$ & $\mathrm{R}_{\Gamma \cdot \mathrm{H}}$ & $\mathrm{R}_{\mathrm{r}-\mathrm{O}}$ & $\theta_{\mathrm{HCH}}$ & $h v_{1}$ & $h v_{2}$ & $\mathrm{~h} v_{3}$ \\
\hline 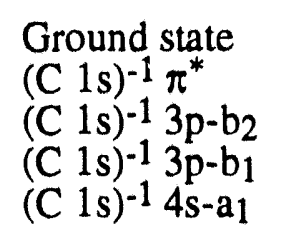 & $\begin{array}{l}1.1171(10) \\
1.037(3) \\
1.09[1.14] \\
1.06[1.17] \\
1.01[1.22]\end{array}$ & $\begin{array}{l}1.2072(5) \\
1.316(5) \\
1.16[1.25] \\
1.18[1.23] \\
1.17[1.24]\end{array}$ & $\begin{array}{l}116^{\circ} 14^{\prime}(6) \\
111^{\circ}(2)\end{array}$ & $\begin{array}{l}345.0 \\
430(5) \\
325(10) \\
381(5) \\
364(5)\end{array}$ & $\begin{array}{l}216.5 \\
160(4) \\
199(8) \\
178(10) \\
227(5)\end{array}$ & $\begin{array}{l}186.0 \\
280(20)\end{array}$ \\
\hline $\mathrm{D}_{2} \mathrm{CO}$ & $\mathrm{R}_{C-\mathrm{D}}$ & $\mathrm{R}_{\mathrm{C}-\mathrm{O}}$ & $\theta_{\mathrm{DCD}}$ & $h v_{1}$ & $h v_{2}$ & $h_{13}$ \\
\hline 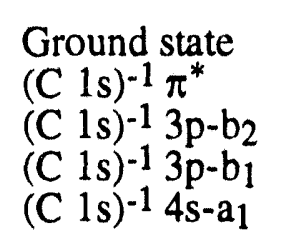 & $\begin{array}{l}1.1130(10) \\
1.091(5) \\
1.06[1.16] \\
1.06[1.17] \\
1.03[1.20]\end{array}$ & $\begin{array}{l}1.2067(5) \\
1.302(5) \\
1.19[1.22] \\
1.19[1.21] \\
1.17[1.25]\end{array}$ & $\begin{array}{l}116^{\circ} 19^{\prime}(6) \\
105^{\circ}(2)\end{array}$ & $\begin{array}{l}254.9 \\
315(5) \\
239(12) \\
268(5) \\
258(12)\end{array}$ & $\begin{array}{l}211.0 \\
179(5) \\
157(10) \\
149(20) \\
134(5)\end{array}$ & $\begin{array}{l}137.1 \\
160(25)\end{array}$ \\
\hline
\end{tabular}


Table IV-4: Isotopic effects on energies $E$ and linewidths W (FWHM) of the (C 1s) ${ }^{-1} 3 s-a_{1}$ and $(\mathrm{O} 1 \mathrm{~s})^{-1} 3 \mathrm{~s}-\mathrm{a}_{1}$ states of formaldehyde. The numbers in parenthesis give the error bars in units of the last digit.

\begin{tabular}{|c|c|c|c|c|}
\hline & \multicolumn{2}{|c|}{ Linewidth (meV) } & \multicolumn{2}{|c|}{ Energy $(\mathrm{eV})$} \\
\hline & $\mathrm{H}_{2} \mathrm{CO}$ & $\mathrm{D}_{2} \mathrm{CO}$ & $\mathrm{H}_{2} \mathrm{CO}$ & $\mathrm{D}_{2} \mathrm{CO}$ \\
\hline $\begin{array}{l}\left(\begin{array}{lll}C & 1 s\end{array}\right)^{-1} 3 s-a_{1} \\
\left(\begin{array}{lll}O & 1 s\end{array}\right)^{-1} 3 s-a_{1}\end{array}$ & $\begin{array}{l}240(20) \\
346(25)\end{array}$ & $\begin{array}{l}180(20) \\
302(25)\end{array}$ & $\begin{array}{l}290.18(1) \\
535.43(1)\end{array}$ & $\begin{array}{l}290.23(1) \\
535.47(1)\end{array}$ \\
\hline
\end{tabular}




\section{FIGURE CAPTIONS:}

Figure IV-1: Overview of the photoionization spectrum of $\mathrm{H}_{2} \mathrm{CO}$ near the carbon is ionization threshold $\mathrm{IP}_{\mathrm{C}-\mathrm{K}}$. The broad resonance above threshold is plotted in the insert on an energy scale which is compressed by a factor of 3 ; the ordinate scale of the inset is the same as that of the main figure.

Figure IV-2: High-resolution spectrum of the (C $1 \mathrm{~s})^{-1} \pi^{*}$ resonance in (a) $\mathrm{H}_{2} \mathrm{CO}$ and (b) $\mathrm{D}_{2} \mathrm{CO}$. The solid line through the data points shows the results of a least-squares fit with Lorentzian functions convoluted by a Gaussian function for instrumental resolution, using Franck-Condon factors to predict the relative peak intensities. The subspectra show the $v_{2}{ }^{\prime}$ vibrational series for different fixed $v_{1}$ ' and $v_{3}$ ' quantum numbers; for detailed explanations, see the text. Note the pronounced changes in vibrational energies upon replacement of $H$ by $D$, indicated in part by the vertical bars.

Figure IV-3: Core-excited Rydberg states below the carbon $\mathrm{K}$-edge in (a) $\mathrm{H}_{2} \mathrm{CO}$ and (b) $\mathrm{D}_{2} \mathrm{CO}$. The solid line through the data points shows the results of a least-squares fit with Lorentzian functions convoluted by a Gaussian function for instrumental resolution. The component peaks are given by solid lines if their energies show no isotopic shift; these represent fundamental transitions to the ground vibrational level of an excited electronic state. Dotted and dashed lines are used for states in which the $v_{2}$ and $v_{1}$ modes, respectively, are excited; these states decrease in energy upon replacement of $H$ by $D$. The dash-dotted component represents the fundamental transition for the $(\mathrm{C} 1 \mathrm{~s})^{-1} 3 \mathrm{~s}-\mathrm{a}_{1}$ state, which exhibits a strong isotopic effect.

Figure IV-4: Spatial distributions of the molecular orbitals in ground-state $\mathrm{H}_{2} \mathrm{CO}$. Reprinted, with permission, from Ref. [12]. 
Figure IV-5: Photoionization spectrum of formaldehyde near the oxygen 1s ionization threshold. The spectra of $\mathrm{H}_{2} \mathrm{CO}$ and $\mathrm{D}_{2} \mathrm{CO}$ are seen to be quite similar, except for small differences in the $(\mathrm{O} 1 \mathrm{~s})^{-1} 3 \mathrm{~s}-\mathrm{a}_{1}$ transition. Note the break in the energy scale, which removes an intermediate part of the spectrum containing no absorption features. 


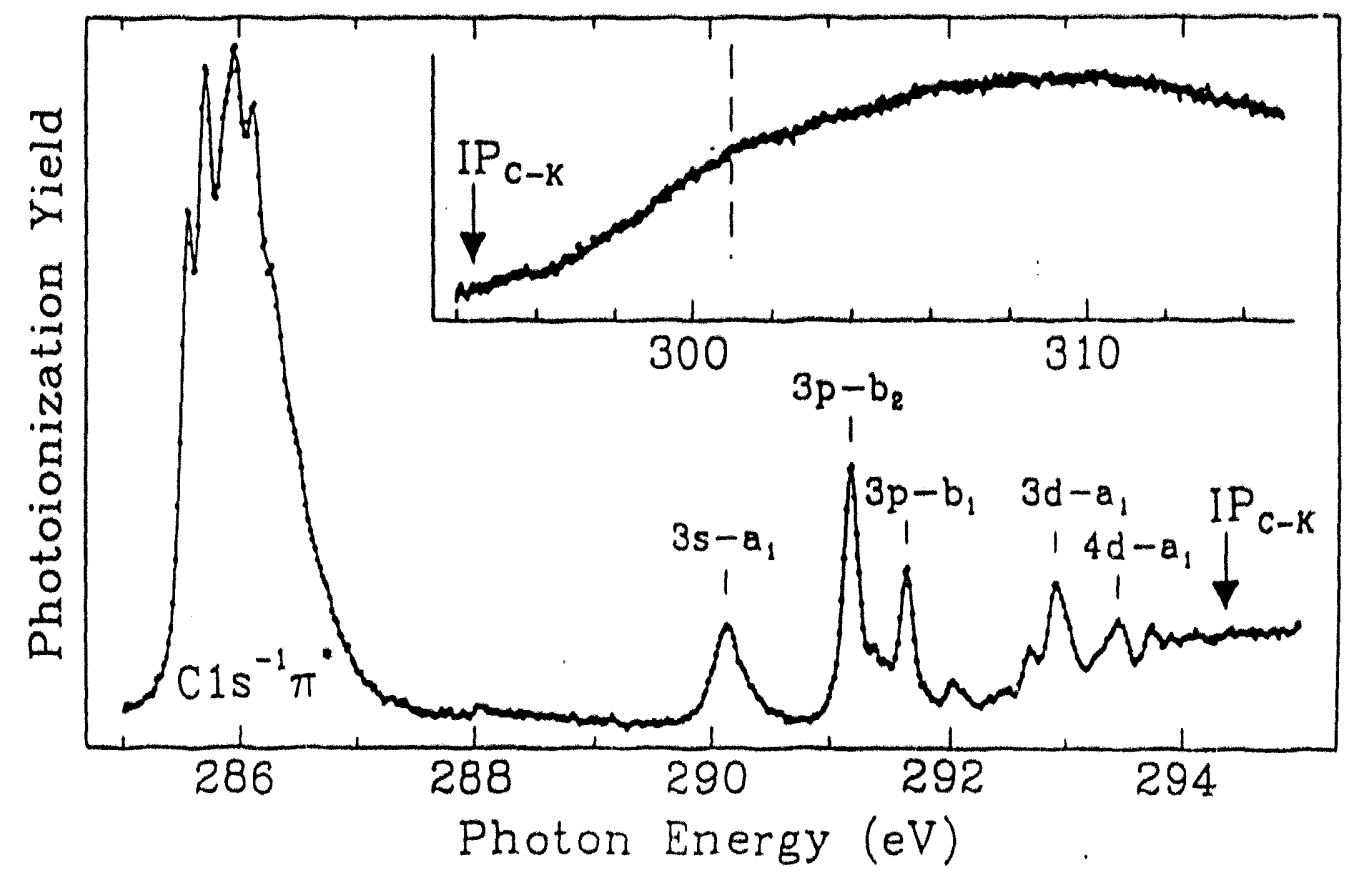

Figure IV-1 


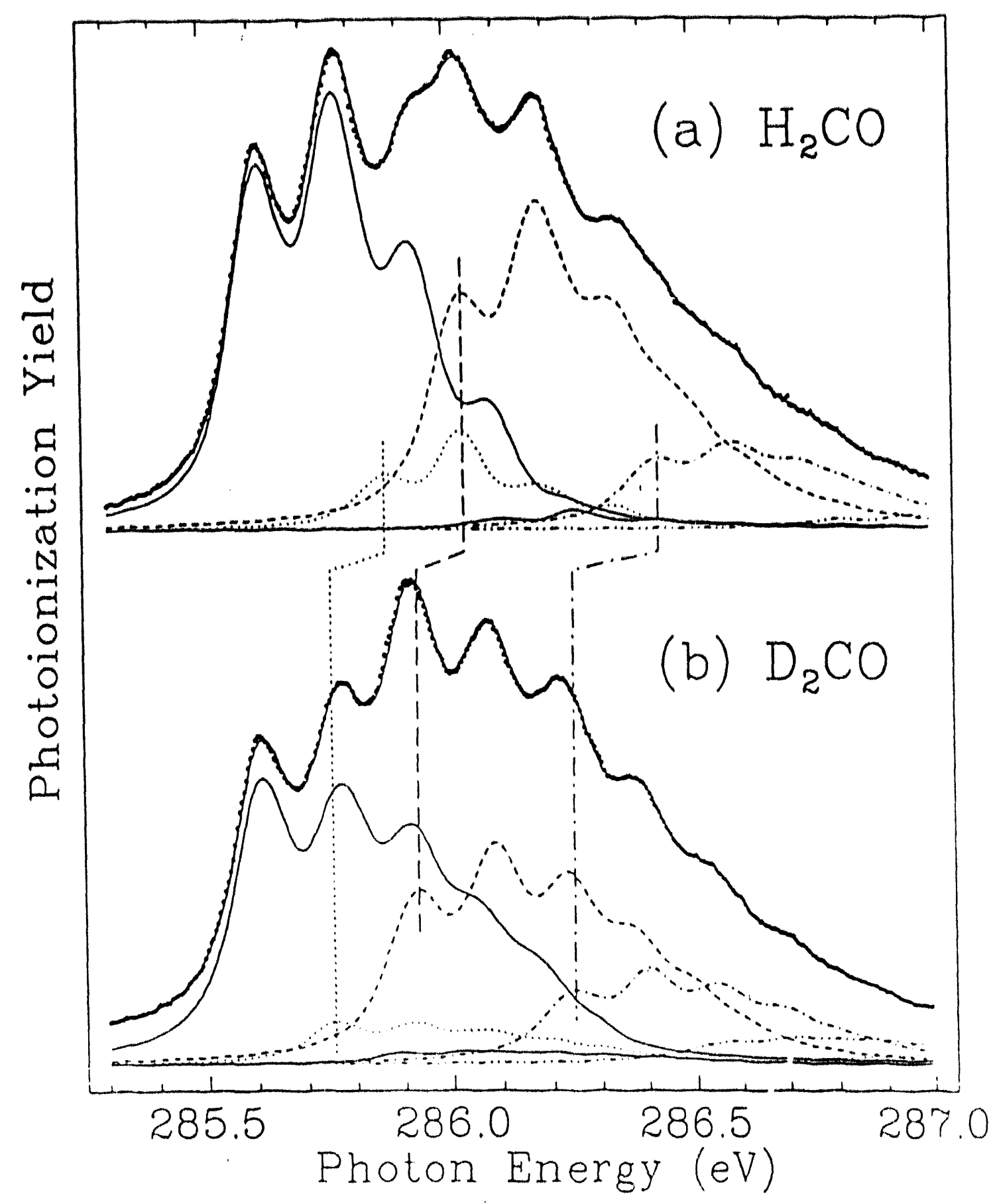

Figure IV-2 


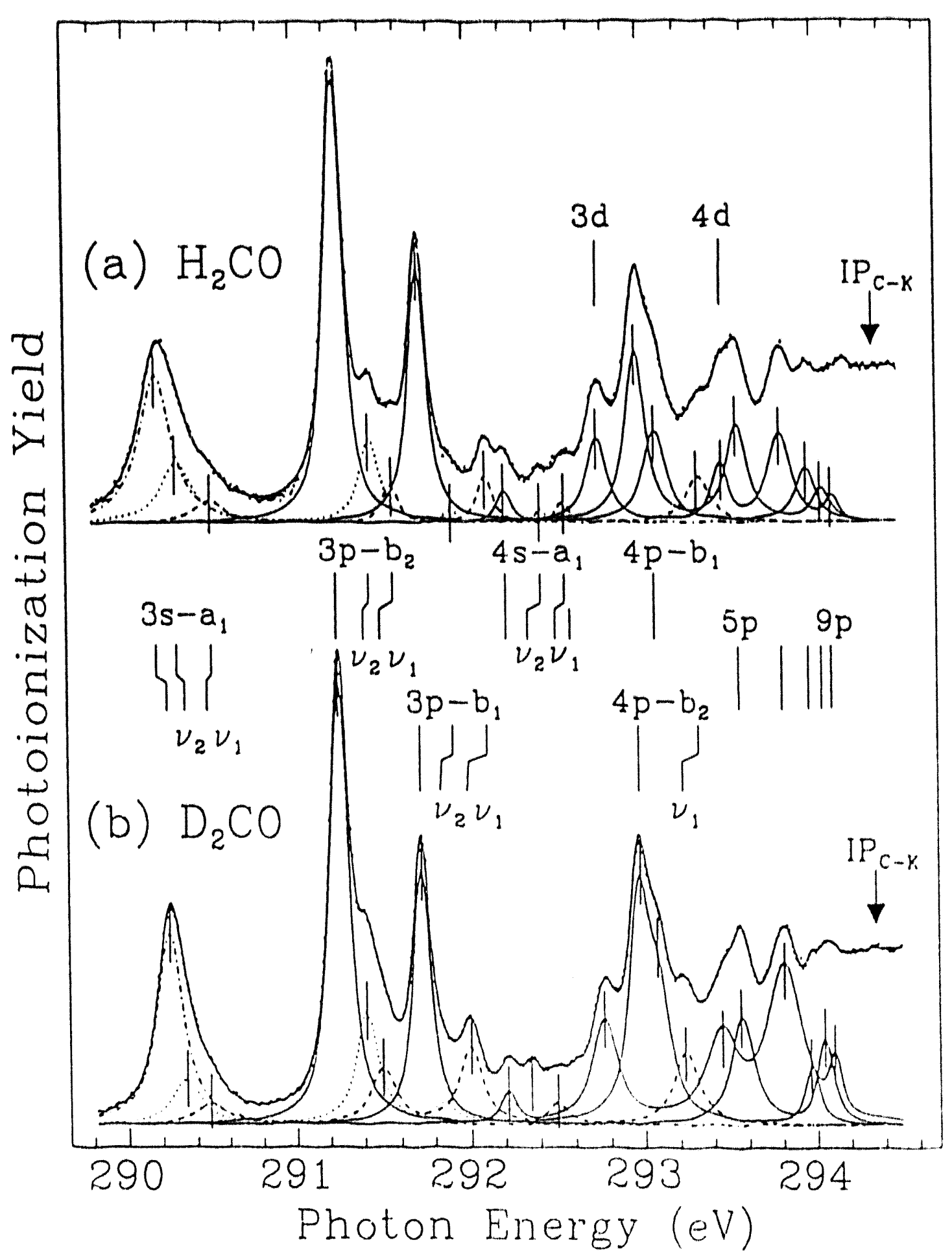

Figure IV-3 

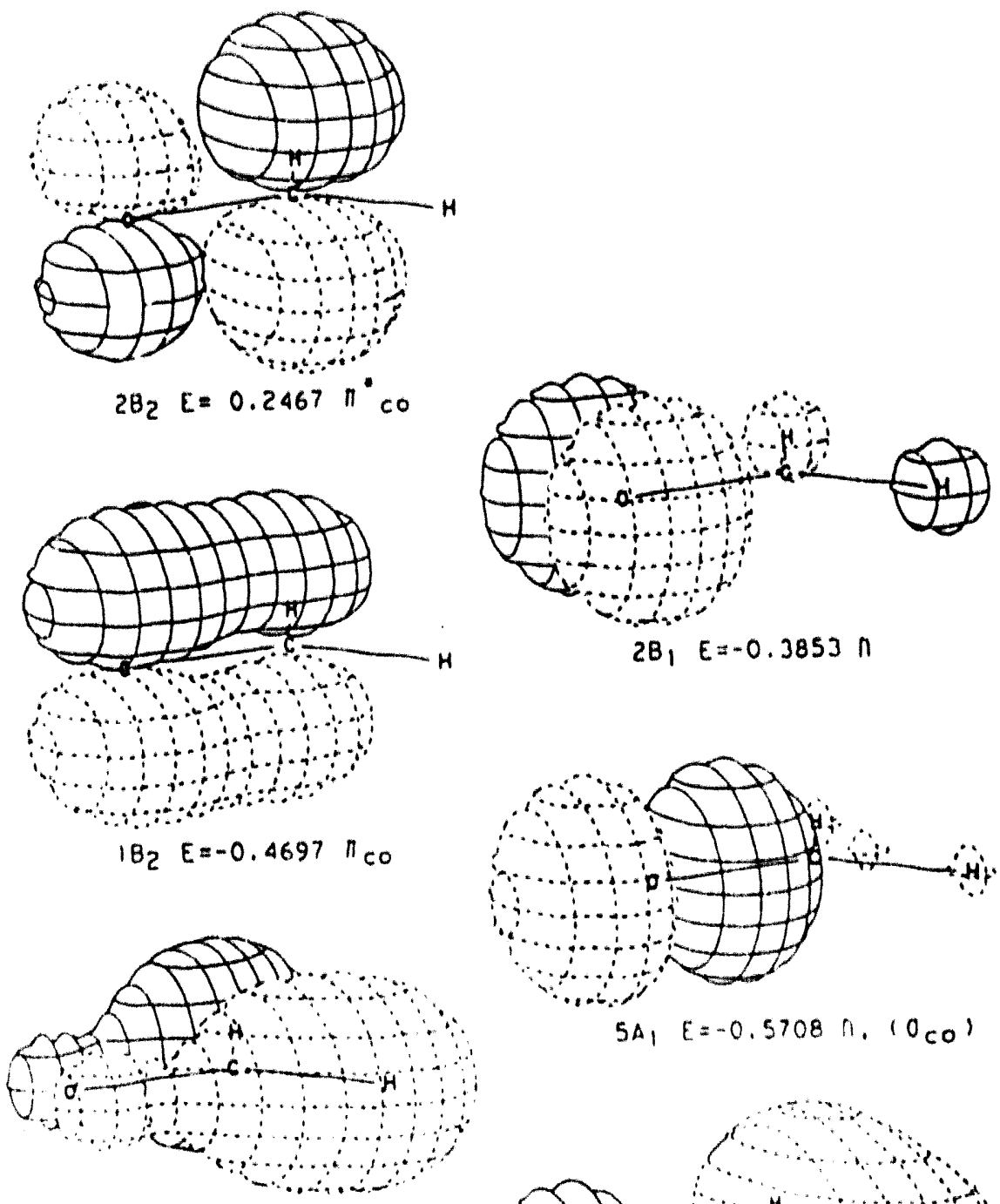

$18, \varepsilon=-0.6745 \mathrm{ncm}$,
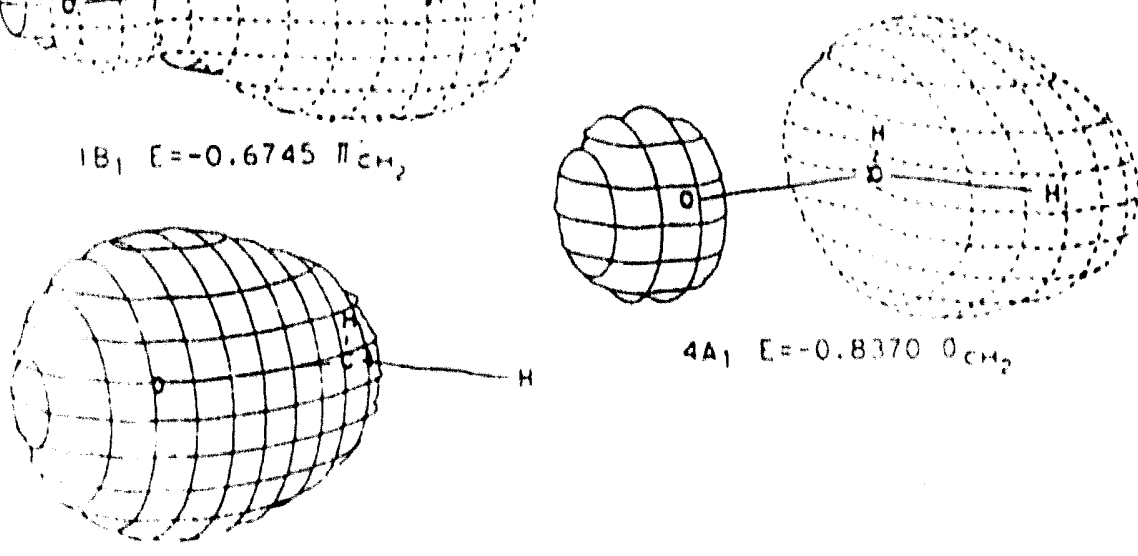

$4 A, E=-0.877000^{\circ}$.

$3 A_{1} E=-1.36930$ CO

Figure IV-4 


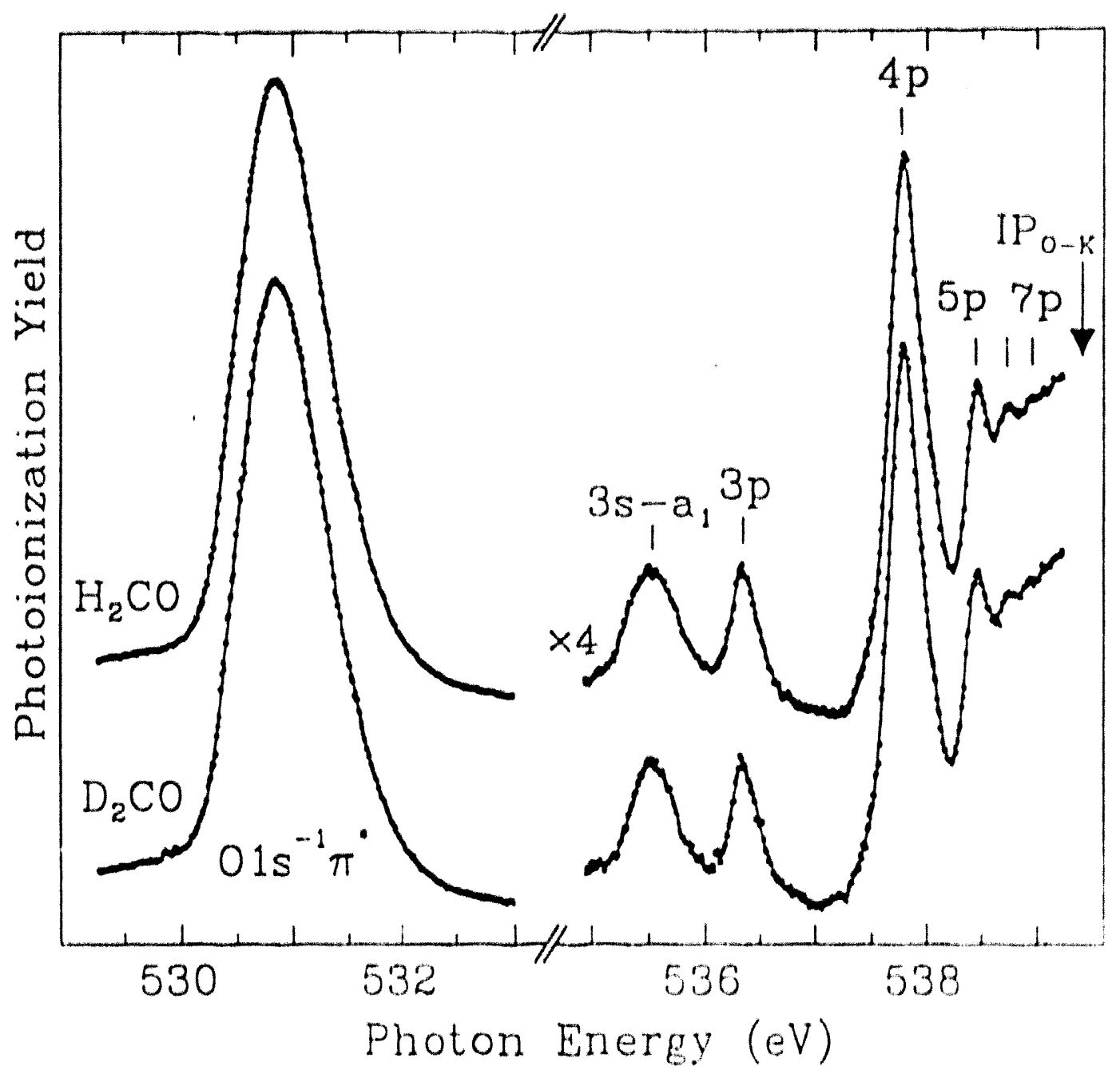

F1gure IV-5 


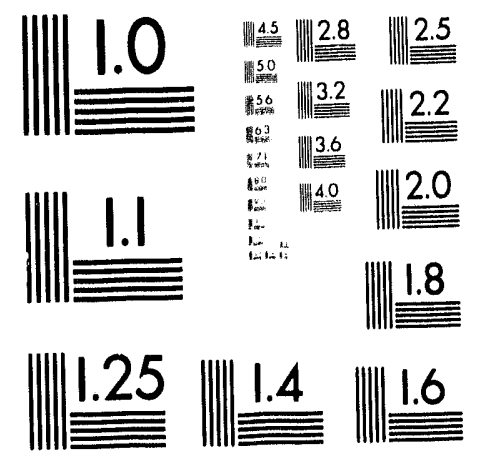



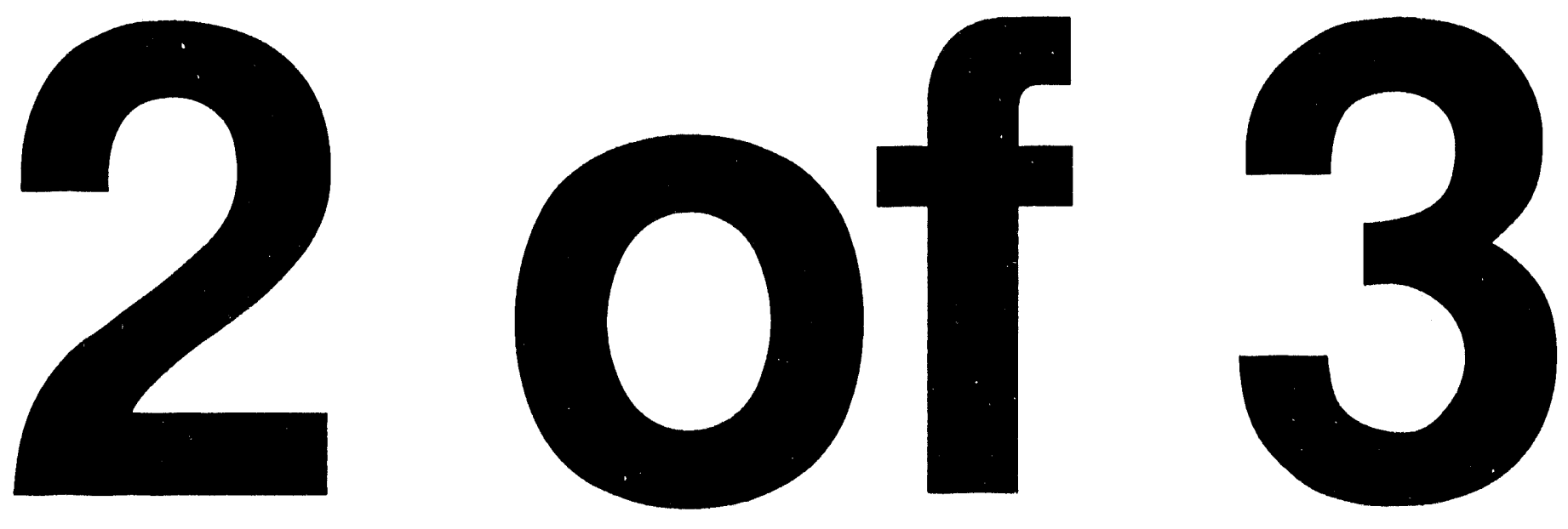


\section{CHAPTER V: HIGH-RESOLUTION MEASUREMENTS OF NEAR-EDGE RESONANCES IN THE CORE-LEVEL PHOTOIONIZATION SPECTRA OF SF6}

\section{V.A. Introduction}

Resonances in XANES spectra arise from the promotion of a core electron into a virtual orbital. The final state of the system is commonly described by referring to the character of the newly occupied orbital. For some systems, the final state may be classified as an inner- or outer-well state. This scheme refers to the atomic partial-wave potential energy diagram for an electron of angular momentum $\ell>0$ (Fig. V-1). The centrifugal contribution to the potential energy may combine with the electrostatic contributions to produce a potential barrier as shown. In molecules, the electrons around the outer atoms may produce a similar barrier along the bond directions. In $\mathrm{SF}_{6}$, this double-well potential is particularly well-developed, because the six $\mathrm{F}$ atoms surround the $\mathrm{S}$ atom. Indeed, because of its chemical stability, small size, and large coordination, $\mathrm{SF}_{6}$ provides the best example of a molecular potential barrier. Virtual orbitals with a spatial distribution primarily inside the potential barrier are known as inner-well orbitals. If an electron is promoted into such a valence orbital, the system is said to be in an inner-well state. Dipoleallowed transitions to such states are normally quite intense in XANES spectra. If the virtual orbital is located mainly in the outer well, the transition is to a Rydberg state. These transitions are weak, as a result of poor spatial overlap between the core orbitals and the outer-well orbitals. Transitions to bound inner-well states lie at energies lower than transitions to Rydberg states. The Rydberg resonances have a series of energies which converge on the absorption edge from below. Transitions to quasi-bound inner-well states are referred to as shape resonances and occur at energies above the absorption edge. 
This chapter presents photoionization spectra of gaseous $\mathrm{SF}_{6}$ at the sulfur $\mathrm{L}_{2,3}$ and fluorine $\mathrm{K}$ edges. Previous photoabsorption $[1,2,3,4,5,6,7]$ and EELS $[8,9]$ measurements showed intense resonances both above and below these thresholds, arising from the promotion of a core electron into a virtual molecular orbital with large amplitude within the molecule. Very weak Rydberg resonances were also detected just below the sulfur $L_{2,3}$ edges, but were not completely characterized. In the present work, improved resolution and statistics resulted in the detection of additional members of the Rydberg series below the sulfur $L_{2,3}$ edges. This allows an unambiguous assignment of peaks in this region, including the confirmation of vibronically-coupled excitations and a $d$ symmetry Rydberg series. Quantum defects, spin-orbit splitting, and the $\mathrm{L}_{2,3}$ ionizationthreshold energies were determined from a least-squares fit of the spectrum. Vibrational structure was resolved for the first time in several Rydberg states. A Franck-Condon analysis yielded the vibrational spacing and S-F bond length for the core-excited ( $\left.2 \mathrm{p}_{3} / 2\right)^{-1}$ $4 \mathrm{~s}$ state. Multiplet splitting was observed for the (S $\left.2 \mathrm{p}_{3 / 2}\right)^{-1} 3 \mathrm{~d}$ state. The derived natural linewidths of the (S 2p $3 / 2)^{-1} 3 d-t_{2 g}$, (S 2p3/2) $)^{-1} 5 s$ and (S 2p $\left.3 / 2\right)^{-1} 4 d$ Rydberg states were found to be strikingly narrower than those of the $(S 2 p)^{-1} \mathrm{a}_{1 \mathrm{~g}}$ inner-well resonances.

High signal-to-noise ratios in the spectra of the more intense resonances allowed lineshape analyses. Non-Lorentzian broadening of the $(\mathrm{S} 2 \mathrm{p})^{-1} \mathrm{a}_{1 \mathrm{~g}}$ states is attributed to unresolved vibrational structure. The peaks assigned to the $(S 2 p)^{-1} \mathrm{t} 2 \mathrm{~g}$ states have large Lorentzian components, suggesting that vibrational effects are relatively small for these shape resonances. Significant intensity very close in energy to the fluorine K edge could not be fitted by a simple edge-jump model and was assigned to a manifold of unresolved resonances. A previously unobserved resonance of large width and low intensity was detected above both the sulfur $L_{2,3}$ and fluorine $K$ edges. 


\section{V.B. Experimental}

The SX700/II monochromator with the 1221 line/mm grating was employed for all measurements, except as noted below. Using this grating, some spectra were recorded using the special low-emittance storage-ring conditions (small-source mode) to allow better resolution. With normal emittance at BESSY, resolution in the first order of diffraction is $\approx 45 \mathrm{meV}$ (Gaussian Full Width at Half Maximum [FWHM]) at the sulfur $\mathrm{L}_{2,3}$ edges and $\approx 310 \mathrm{meV}$ at the fluorine $\mathrm{K}$ edge. In the small-source mode, the resolution improves to $\approx 30 \mathrm{meV}$ at the sulfur $L_{2,3}$ edges. Additional measurements were made using a 2442 line/mm grating in the first order of diffraction with normal emittance conditions, providing a resolution of $=33 \mathrm{meV}$ at the sulfur $\mathrm{L}_{2,3}$ edges with relatively high flux. The synchrotron radiation beam passed through a $1500 \AA$ thick $\mathrm{Al}(1 \% \mathrm{Si})$ window into an interaction region containing $\mathrm{SF}_{6}$ gas (Messer Griesheim $\mathrm{GmbH}, 99.9 \%$ ), which was maintained at pressures ranging from $60 \mathrm{mtorr}$ to $175 \mathrm{mtorr}$.

\section{V.C. Overview of Results}

Fig. V-2 shows the photoionization spectrum of the octahedral molecule $\mathrm{SF}_{6}$ near the sulfur $L_{2,3}$ ionization thresholds. The double-peaked structure 1,2 at $172-174 \mathrm{eV}$ arises from the promotion of a sulfur $2 p$ electron to an unoccupied molecular orbital of $a_{1 g}$ symmetry. This absorption feature is split by the spin-orbit interaction of the $2 p$ core electrons in the final state. A better-resolved spectrum of the (S 2p) $)^{-1} \mathrm{a}_{1 \mathrm{~g}}$ resonances, taken in the small-source mode of BESSY, is shown in Fig. V-3. A more intense doublet 3,4 appears at $182-184 \mathrm{eV}$, just above the sulfur $\mathrm{L}_{2,3}$ thresholds in Fig. V-2. It is assigned to the promotion of a single sulfur $2 p$ electron to a quasi-bound orbital of $\mathrm{t}_{2 \mathrm{~g}}$ symmetry, with spin-orbit splitting again accounting for the double-peaked structure. This is a well-known example of a molecular shape resonance, i.e. the promotion of an electron into a one- 
electron continuum state which is resonantly enhanced within the molecule by the molecular potential $[10,11]$. The broad absorption peak 5 at $196 \mathrm{eV}$ is assigned to the excitation of a resonance of $e_{\mathrm{g}}$ symmetry. This state is a shape resonance which may couple to several other continuum channels and may also include contributions from autoionizing multielectron excitations [12]. The small peak 6 at $205 \mathrm{eV}$ has been described as a multi-electron excitation [8] and as an EXAFS resonance [9], but it is not yet fully understood. An even smaller resonance, peak 7 at $209 \mathrm{eV}$, which appears to be a doublet, is observed here for the first time and may also be a multi-electron excitation. Note that the inset data of Fig. V2 showing peaks 6 and 7 in greater detail were taken using the 2442 line/mm grating. The known complexity of the $e_{g}$ resonance suggests that the $t_{2 g}$ resonance may also be more than a simple one-electron excitation shape resonance. An autoionizing state with two excited electrons has been predicted near the energy of the $t_{2 g}$ resonance [12]. A lowerenergy $t_{2 g}$ resonance of this molecule is known to couple to several continuum channels upon excitation at $23 \mathrm{eV}$ photon energy [13]. A similar process is possible at the $\mathrm{L}_{2,3}$ edges, as discussed below.

Fig. V-4 shows the spectrum in the $177-182 \mathrm{eV}$ range obtained with better resolution and statistics using the 2442 line/mm grating. The weak fine structure detected in the spectrum of Fig. V-2 is clearly resolved here. Many peaks are present; most are readily assigned to one-electron excitations into Rydberg orbitals. These Rydberg states correspond to those of atomic sulfur, the central atom, and are labeled as such. The s- and d-symmetry Rydberg states form two overlapping series, each of which is split into two series by the spin-orbit interaction of the core hole. The resulting four series overlap to give the complicated structure observed. Such an assignment leaves the features E, F, and $\mathrm{G}$ around $178.2 \mathrm{eV}$ unexplained. They are assigned to a vibronically-coupled transition to the (S $\left.2 \mathrm{p}_{3 / 2}\right)^{-1} 4 \mathrm{p}$ Rydberg state. In addition, the region from 177 to $179 \mathrm{eV}$ is characterized by some broad intensity above background (shaded area), which is attributed to a vibronically-coupled transition to a virtual orbital with $t_{1 u}$ symmetry . 
Fig. V-5 shows the photoabsorption spectrum of $\mathrm{SF}_{6}$ near the fluorine $\mathrm{K}$ ionization threshold. Peak 8 at $689.0 \mathrm{eV}$ is assigned to a transition to the $\mathrm{a}_{1 \mathrm{~g}}$ unoccupied molecular orbital, as seen at the sulfur $\mathrm{L}_{2,3}$ edges. The following peaks 9,10 , and 11 , below and just above the edge jump at $695.7 \mathrm{eV}$, are assigned to transitions to a number of $\mathrm{t}_{1 \mathrm{u}}$ virtual orbitals. Peaks 12 and 13, at $699.9 \mathrm{eV}$ and $713.3 \mathrm{eV}$, are excitations to $\mathrm{t}_{2 \mathrm{~g}}$ and $\mathrm{eg}$ shape resonances, respectively, which are analogous to those seen at the sulfur $\mathrm{L}_{2,3}$ edges. Peaks 14 and 15, at $722 \mathrm{eV}$ and $727 \mathrm{eV}$, are probably multi-electron excitations analogous to peaks 6 and 7 in Fig. V-1. Note that dipole-allowed transitions into orbitals of both $g$ and $\mathrm{u}$ parity are observed here, arising from the combination of the six fluorine $1 \mathrm{~s}$ atomic orbitals into the $2 \mathrm{a}_{1 \mathrm{~g}}, 1 \mathrm{e}_{\mathrm{g}}$, and $1 \mathrm{t}_{1 \mathrm{u}}$ molecular orbitals of the initial state. Fig. V-6 shows a level diagram of the core-excited states of $\mathrm{SF}_{6}$, based upon the energies and assignments given above and in Tables $\mathrm{V}-1,2,3$.

\section{V.D. Sulfur $L_{2,3}$ thresholds: Inner-well resonances}

\section{Spin-orbit interaction and exchange interaction}

Nearly all features observed near the sulfur $\mathrm{L}_{2,3}$ thresholds clearly display a doublet structure, with a splitting of $\approx 1.2 \mathrm{eV}$. This is explained by the spin-orbit interaction of the core electrons in the final state. According to Hund's rule, the ( $\left.2 \mathrm{p}_{1 / 2}\right)^{-1}$ configuration will have higher energy than the ( $\left.2 \mathrm{p}_{3} / 2\right)^{-1}$ configuration. The value of the splitting observed here agrees with the measured splittings in $\mathrm{x}$-ray photoelectron spectra (XPS) of

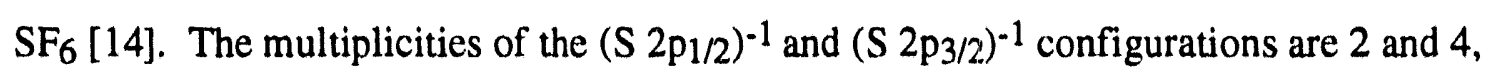
respectively. A simple spin-orbit model of the core-excited final state would predict a

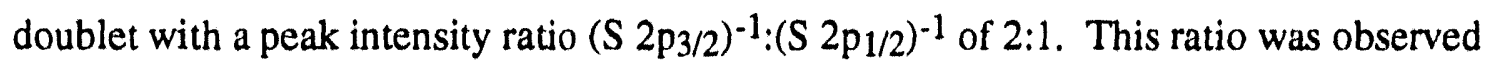
in the XPS spectrum [15], but is not observed for the $\left(S_{2} p_{1 / 2,3 / 2}\right)^{-1} a_{1 g}$ and $\left(S_{2} 2 p_{1 / 2,3 / 2}\right)^{-1}$ 
$\mathrm{t}_{2 \mathrm{~g}}$ photoabsorption resonances. In fact the ratios for these resonances, obtained from least-squares fits of the spectrum in Fig. V-2, are both less than unity (see Table V-1).

This reversal of the intensity ratio can be explained by including the effects of exchange interaction between the core hole and the excited electron $[10,16]$. The (S

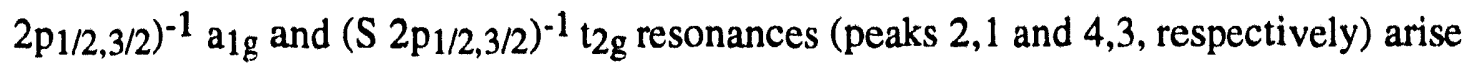
from one-electron transitions into unoccupied molecular orbitals spatially localized within the molecule. An electron excited from a core hole into such an inner-well state will have considerable spatial overlap with the remaining sulfur core electrons. The resulting (core hole)-(excited electron) exchange interaction will be significant in comparison to the spinorbit interaction. In such a case the $\mathrm{j}-\mathrm{j}$ coupling scheme used above is no longer valid; intermediate coupling must be employed. Theoretical studies of this phenomenon in $\mathrm{SF}_{6}$ and other systems show that the main effect of intermediate coupling is to decrease the ( $\left.\mathrm{S} 2 \mathrm{p}_{3 / 2}\right)^{-1}$ to $\left(\mathrm{S} 2 \mathrm{p}_{1 / 2}\right)^{-1}$ intensity ratio [17]. In the limit where exchange coupling completely dominates over spin-orbit coupling, the intensity ratio becomes zero because the lower-energy peak then represents a dipole-forbidden transition to a triplet state.

Calculations also predict that intermediate coupling has little effect on the energy splitting of the doublet [17], in agreement with the present observations (see Tables V-1 and V-2). The observed spin-orbit splittings of the (S 2 $\left.\mathrm{p}_{1 / 2,3 / 2}\right)^{-1} \mathrm{a}_{1 \mathrm{~g}}$ and $\left(\mathrm{S}_{2} \mathrm{p}_{1 / 2,3 / 2}\right)^{-1} \mathrm{t}_{2 \mathrm{~g}}$ doublets $(1.17 \mathrm{eV})$ are only slightly less than those of the Rydberg states $(1.20 \mathrm{eV})$, where the exchange interaction is expected to be small because of the limited overlap of the Rydberg orbitals with the core hole.

\section{Lineshapes}

\section{a. (S 2p)-1 a1g resonances}




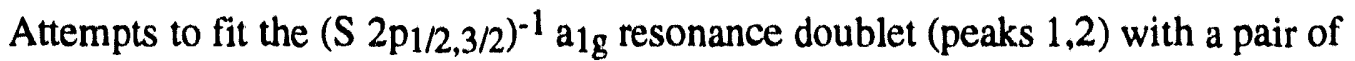
Voigt functions based on reasonable values of the Lorentzian natural linewidth and the Gaussian instrumental width were unsuccessful. The a1g resonance therefore appears to be broadened by non-lifetime effects. An earlier analysis [9] suggested that multiplet splitting within this resonance might lead to observable peak splittings, in analogy to those observed for the $(S 2 p)^{-1} b_{1}{ }^{*}$ resonance in the sulfur $L_{2,3}$ spectrum of $\mathrm{SO}_{2}$ [18]. This turns out to be unlikely for the (S 2p)-1 a $1 \mathrm{~g}$ transitions in $\mathrm{SF}_{6}$, as shown by the following grouptheoretical analysis. In the octahedral symmetry of the $\mathrm{SF}_{6}$ molecule, there are dipoleallowed transitions only to electronic states of $\mathrm{T}_{1 \mathrm{u}}$ symmetry. By multiplying the spatial and spin characters of the $a_{1 g}$ electron and the sulfur $2 p$ core hole, the possible electronic states corresponding to the (S $2 p)^{-1}$ alg configuration are determined (see the discussion in Sec. VI.E.1 ). They are $A_{1 u}, E_{u}, 2 T_{1 u}$, and $T_{2 u}$; thus there are only two dipole-allowed transitions, corresponding to the two observed peaks. Note that weak transitions to the $E_{u}$ and $T_{2 u}$ states are possible through vibronic coupling, while there is no suitable vibrational mode to couple the $A_{1 u}$ state. Therefore small contributions from vibronically-

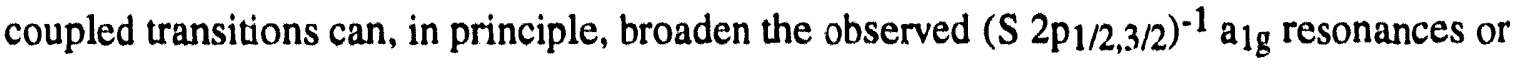
lead to observable splittings. However, based upon the relative intensity of the vibronically-coupled (S $2 p)^{-1} t_{1 u}$ transitions (see Sec. V.D.3), observable effects from transitions to these dipole-forbidden states seem unlikely.

Instead, the non-Lorentzian broadening probably arises from extensive unresolved vibrational excitations in the final state, resulting in an overlapping manifold of peaks. Such excitations are expected, because a non-bonding core electron has been promoted into an anti-bonding orbital. Semi-empirical calculations [19] for the ground state of the octahedral molecule $\mathrm{ClF}_{6}$, which is the equivalent-core molecule to S-core-excited SF6, predict a bond length of $1.63 \AA$ for $\mathrm{Cl}-\mathrm{F}$ compared to $1.561 \AA$ in ground-state $\mathrm{SF}_{6}[20]$. Such a large change in bond length could produce the extensive vibrational excitation suggested here. The only dipole-allowed vibrational excitation is the single a $1 \mathrm{~g}$ symmetric- 
stretch mode, $v_{1}$, (with ground-state frequency $h v_{1} "=96 \mathrm{meV}$ ) [21]. Vibrational fine structure was not observed in this study, even in the spectra taken in the small-source mode of BESSY, with a resolution of $\approx 30 \mathrm{meV}$ (see Fig. V-3). The low-energy shoulders of the (S 2 $\left.\mathrm{p}_{3 / 2}\right)^{-1} \mathrm{a}_{1 \mathrm{~g}}$ peak, which were proposed in Ref. [9], were not observed here in measurements with higher resolution and better statistics. There are slight indications of irregularities in several regions of the (S 2p $)^{-1} \mathrm{a}_{1 \mathrm{~g}}$ lineshape, however, which are not completely convincing in the present data, but suggest that measurements with better spectral resolution and very good statistics might resolve vibrational fine structure in the (S $2 \mathrm{p})^{-1} \mathrm{a}_{1 \mathrm{~g}}$ resonances. This would be particularly desirable considering the unusual results of the Franck-Condon analysis given below. The difficulty in observing fine structure is presumably a consequence of the large natural linewidths of the individual vibrational states. Those natural linewidths are estimated to be $>190 \mathrm{meV}$, FWHM; smaller linewidths would have produced resolved fine structure, assuming a vibrational spacing of $>90 \mathrm{meV}$. Assuming a vibrational spacing of $127 \mathrm{meV}$, as derived below, predicts a natural linewidth of $>230 \mathrm{meV}$.

A Franck-Condon (FC) analysis [22] of the (S 2p- $)^{-1} \mathrm{a}_{1 \mathrm{~g}}$ resonance lineshape was performed, based on harmonic oscillator potentials and Voigt lineshapes. Even without resolved vibrational structure in the spectrum, the FC approach can be used to fit the observed intensity envelope, although the results will be less reliable than those of fits to resolved progressions. The results of the fit are plotted with the small-source data in Fig. V-3. A good fit to the lineshape was obtained only by using an excited-state vibrational spacing greater than that of the ground state $\left(h v^{\prime} \approx 127 \mathrm{meV} v s . h v^{\prime \prime}=96 \mathrm{meV}\right.$, where primes indicate excited state parameters and double primes refer to the ground state). The derived bond length $\left(R^{\prime} \approx 1.62 \AA\right)$ was close to the prediction from the equivalent-core molecule $\mathrm{ClF}_{6}$, but an increase in bond length accompanied by an increase in the associated vibrational stretch frequency is difficult to understand. (Note that there is only one totallysymmetric vibrational mode in this molecule, so the normal-mode composition arguments 
of Chapter IV do not apply here.) In the harmonic oscillator approximation used here, equal contractions and extensions of the bond have equivalent effects on the $\mathrm{FC}$ profile of transitions originating in the ground vibrational state. Thus the fit is also consistent with a decrease in the bond length $\left(\mathrm{R}^{\prime} \approx 1.50 \AA\right)$; this, however, is hard to reconcile with the calculated bond length for $\mathrm{ClF}_{6}$. Also, the $\mathrm{a}_{1 \mathrm{~g}}$ orbital is expected to have antibonding character; populating it should weaken the S-F bonds. Further studies are necessary to gain a better understanding of this excited state.

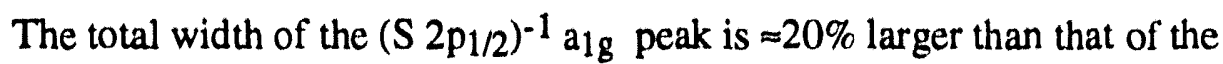
$\left(\mathrm{S} 2 \mathrm{p}_{3 / 2}\right)^{-1}$ a1g peak (see Table V-1). The shapes of the FC envelopes for the two resonances should be identical, except for the effect of the natural linewidths of the individual vibrational states and a very small difference from the fourth-power dependence of the FC factors on excitation energy. The natural linewidths resulting from the FC fit based on this assumption $\left(\Gamma\left[\left(S 2 \mathrm{p}_{3 / 2}\right)^{-1} \mathrm{a}_{1 \mathrm{~g}}\right] \approx 240 \mathrm{meV}(\mathrm{FWHM}), \Gamma\left[\left(\mathrm{S}_{2} \mathrm{p}_{1 / 2}\right)^{-1} \mathrm{a}_{1 \mathrm{~g}}\right] \approx\right.$ $390 \mathrm{meV}$ ) are both quite large, especially in comparison with the exceptionally narrow natural linewidths (e.g. $35 \mathrm{meV}$ ) measured in the Rydberg region of this spectrum (see Section V.B.3). The large difference between the derived natural linewidths of the $\left(\mathrm{S} 2 \mathrm{p}_{1 / 2}\right)^{-1} \mathrm{a}_{1 \mathrm{~g}}$ and $\left(\mathrm{S} 2 \mathrm{p}_{3 / 2}\right)^{-1} \mathrm{a}_{1 \mathrm{~g}}$ states indicates different decay rates of the two excited states. The higher-energy $\left(S_{2} 2 \mathrm{p}_{1 / 2}\right)^{-1} \mathrm{a}_{1 \mathrm{~g}}$ resonance may autoionize faster because it has more decay channels, perhaps including decay through the $\left(S 2 p_{3 / 2}\right)^{-1}$ a $1 \mathrm{~g}$ resonance. An analogous difference in linewidths has not been observed in the XANES spectra of rare gases in the soft x-ray energy range (see e.g. Ref. [23], Figs. 3,4,5). In these atomic systems, however, there are no vibrational or rotational levels to take up excess electronic energy, so that an inter-system crossing between non-degenerate electronic states is not possible. This effect might also be a further manifestation of intermediate coupling. Specifically, the (S 2p3/2)-1 alg state acquires some triplet-state character through exchange interaction. In the triplet state, the excited electron has its spin parallel to the unpaired sulfur $2 p$ electron. The excited electron will avoid the unpaired electron and thus also avoid 
the core hole, which has the same spatial distribution. The result is a reduced probability for an autoionization process involving the excited electron (which may be the dominant decay process; see Sec. V.E.3). The (S $\left.2 \mathrm{p}_{3 / 2}\right)^{-1} \mathrm{a}_{1 \mathrm{~g}}$ peak narrows to reflect the increased lifetime of this state. The $\left(\mathrm{S}_{2} \mathrm{p}_{1 / 2}\right)^{-1} \mathrm{a}_{1 \mathrm{~g}}$ state has no triplet character and thus has a shorter lifetime. This explanation is consistent with the absence of the described effect in the XANES spectra of rare gases [23], since those systems do not exhibit notable intermediate coupling effects.

\section{b. $(S 2 p)^{-1} t_{2 g}$ resonances}

A least-squares fit of the lineshapes of the $\left(S 2 p_{3 / 2,1 / 2}\right)^{-1} t_{2 g}$ resonances (peaks 3,4) using Voigt functions showed significant Lorentzian contributions. A good fit to the data was obtained using a single Gaussian width of $270 \mathrm{meV}$ (FWHM) and Lorentzian widths

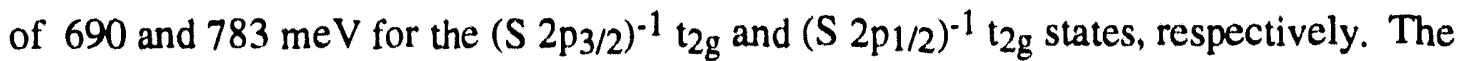
bandwidth of radiation contributes $\approx 45 \mathrm{meV}$ to the Gaussian width, so that most of the Gaussian width observed represents real effects in the molecule. Some of the intensity of this resonance may be from a two-electron excitation to a bound state, which could be Gaussian-broadened by vibrational excitations. Gaussian-like broadening could also result from a slight offset of two or more overlapping resonances (e.g. one shape resonance state and one bound state or two shape resonance states). The $t_{2 g}$ resonance was observed, however, in the direct photoelectron spectrum of the sulfur $2 \mathrm{p}$ levels, indicating that the resonance must be at least partly due to a shape resonance [12]. If the observed lineshape were determined mainly by two-electron excitations, then the shape resonance contribution would have an extraordinarily narrow linewidth $(<0.3 \mathrm{eV})$. The more likely possibility is that the lineshape is partly or completely determined by the shape resonance.

The relatively large Lorentzian contribution to the lineshape of the $(S 2 \mathrm{p} 3 / 2,1 / 2)^{-1} \mathrm{t}_{2 \mathrm{~g}}$ shape resonance is somewhat unexpected. The implication is that the vibrational effects 
upon this resonance differ from those of other known shape resonances. Shape resonances are generally believed to induce some vibrational excitation of the final-state core-ionized molecule [24]. While this vibrational structure will probably not be resolved, because of the large natural linewidths of the shape resonance, the resulting photoabsorption lineshapes should show non-Lorentzian broadening. Ground-state vibrational (zero-point) motion may also couple to the transition, creating broad asymmetric lineshapes [24]. The relatively small Gaussian contribution to the lineshape observed here indicates that both of these vibrational effects are small in the $\mathrm{t}_{2 \mathrm{~g}}$ shape resonance. A study of the ionic fragmentation pattern obtained from the decay of this excited state also indicated that there is little vibrational excitation in this state [25].

The strong Lorentzian character of the lineshape is consistent with a rapidly decaying quasi-bound shape resonance state, but is also consistent with an alternative interpretation of this feature as a resonance in more than one continuum channel. Core-hole lifetimes are generally longer than the lifetime of quasi-bound shape resonance states. This can be seen directly by comparing the narrow photoelectron peaks at resonance to the wide photoabsorption peaks [26]. The $\mathrm{t}_{\mathrm{g}}$ resonance may differ, such that the autoionization decay of the core hole is faster than the departure of the quasi-bound electron. This could explain the large observed Lorentzian component of the lineshape, but would also describe the $t_{2 g}$ peak not really as a shape resonance but rather as a quasi-bound one-electron excited state that decays rapidly by an Auger-like autoionization process. This would give the same final state as a direct photoelectron transition from a subshell less tightly bound than sulfur $2 p$. In general, this type of continuum-continuum coupling is observed as a resonance in one photoelectron channel at the same photon energy as a shape resonance in another channel. Such an inter-channel resonance was observed in the valence photoelectron spectra of $\mathrm{SF}_{6}$ and attributed to the $\mathrm{t}_{2 \mathrm{~g}}$ shape resonance of the $1 \mathrm{t}_{2 u}+5 \mathrm{t}_{1 \mathrm{u}}$ channel coupling to the $3 \mathrm{e}_{\mathrm{g}}$ photoelectron channel [13]. This is not a normal shape resonance; the kinetic energy of the photoelectron is not tuned to the shape of the potential. 
The most general model of the ( $\left.2 \mathrm{p}_{1 / 2,3 / 2}\right)^{-1} \mathrm{t}_{2 \mathrm{~g}}$ resonance includes contributions from double excitations, a shape resonance, and continuum-continuum coupling. The results of the ionic fragmentation study [25] suggest that the direct shape resonance dominates. More work is needed, however, for a complete understanding of the observed lineshape.

The lineshape analysis also shows that the $\left(\mathrm{S} 2 \mathrm{p}_{1 / 2}\right)^{-1} \mathrm{t}_{2 \mathrm{~g}}$ resonance, peak 4 , is $\approx 13 \%$ broader than the $\left(\mathrm{S}_{2} \mathrm{p}_{3 / 2}\right)^{-1} \mathrm{t}_{2 \mathrm{~g}}$ resonance, peak 3 . This is consistent with the above results for the widths of the (S $\left.2 \mathrm{p}_{3 / 2,1 / 2}\right)^{-1} \mathrm{a}_{1 \mathrm{~g}}$ resonances. Again it seems that the ( $\mathrm{S}$ $\left.2 \mathrm{p}_{1 / 2}\right)^{-1}$ core hole decays faster than the (S $\left.2 \mathrm{p}_{3 / 2}\right)^{-1}$ core hole.

\section{Vibronic coupling}

Transitions to electronic final states which are not dipole-allowed may still have noticeable intensity through vibronic coupling. If vibrational excitation occurs in nontotally-symmetric vibrational modes such that the final vibrational-electronic (vibronic) state is of a symmetry accessible by a dipole transition, the transition can occur, though usually with low intensity [27]. This phenomenon can explain two features of the spectra shown in Fig. V-4. One feature is the set of three ( $\left.2 \mathrm{p}_{3 / 2}\right)^{-1} 4 \mathrm{p}$ peaks E,F,G mentioned in Section V.C. The other is the broad underlying intensity (shaded area in Fig. V-4) in the photon-energy range $175-179 \mathrm{eV}$. This latter feature is assigned to transitions into one or two inner-well orbitals with $t_{1 u}$ symmetry. Both features involve $t_{1 u}$ symmetry final-state orbitals. Direct excitation of these states is electric-dipole forbidden, but electricquadrupole allowed. Both features have more spectral weight in the EELS spectra of Ref. [8] than in the corresponding photoabsorption spectra. This indicates that the higher order transitions (electric quadrupole, etc.), which may be induced by the EELS excitation process [28], enhance these two spectral features. More recent studies using angleresolved EELS [29] clearly identified, at $177.5 \mathrm{eV}$, a dipole-forbidden, quadrupole-allowed transition to an antisymmetric orbital, assigned to a $t_{1 u}$ inner-well state. In contrast, the 
EELS spectra of Ref. [9] were taken under conditions which suppress higher-order transitions, and the results are quite similar to the present photoionization results. Even in these spectra, where dipole-selection rules apply, transitions to $(S 2 p)^{-1} t_{1} u$ states may be observed if at least one quantum of an antisymmetric vibrational mode is excited. There are two $t_{1} u$ modes and one $t_{2 u}$ mode in $S_{6}$.

This interpretation is also supported by the assignment of the remaining Rydberg structure given below. Attempts to assign peak $\mathrm{E}$ at $178.3 \mathrm{eV}$ as a member of a Rydberg sor $\mathrm{d}$-series led to a poor description of the remaining peaks. The assignment of $E$ instead to a $4 p$ Rydberg state leads to a sensible value of the quantum defect (see Sec. V.E.5).

The existence of $t_{1 u}$ inner-well orbitals in $\mathrm{SF}_{6}$ has been predicted by theoretical work [30,31,32]. Dipole-allowed transitions to these orbitals were observed in $\mathrm{SF}_{6}$ spectra at the fluorine $K$ edge $[8,9]$, as well as the $L_{1}[8,9]$ and $K[6]$ edges of sulfur. Excellent spatial overlap with sulfur $2 p$ electrons is expected, considering the large intensity of the transition to the $t_{1 u}$ orbital below the sulfur $L_{1}$ edge $[8,9]$.

The initially published spectra of the $S L_{2,3}$ region showed only the most intense features of the rich fine structure in the spectrum of Fig. V-4. Several papers $[11,31,33]$ assigned these weakly resolved peaks to transitions to $(S 2 p)^{-1} t_{1 u}$ states. The present work illustrates the success of the Rydberg formula in predicting the energies of the observed transitions. Their assignment as Rydberg states is further supported by their linewidths, which are much smaller than those of the ( $\left.\mathrm{S}_{2} \mathrm{p}_{1 / 2,3 / 2}\right)^{-1}$ inner-well resonances. The $\left(S_{2}\right)^{-1} t_{1 u}$ states anticipated by these early workers are observed here as the broad underlying intensity described above (shaded area in Fig. V-4).

Vibronic coupling between the ground state and a dipole-forbidden electronic final state is actually mediated by one or more dipole-allowed excited electronic states. Such states mix with the dipole-forbidden electronic final state via vibronic interaction [27]. The mixing and thus the vibronically-coupled transition are facilitated in this case by the existence of intense dipole-allowed transitions (the $\mathrm{a}_{1 \mathrm{~g}}, \mathrm{t}_{2 \mathrm{~g}}$, and $\mathrm{e}_{\mathrm{g}}$ resonances) that are 
close in energy to the vibronically-coupled states. Vibronic interaction between these sets of states is symmetry-allowed through the two $t_{1 u}$ or one $t_{2 u}$ vibrational modes of $\mathrm{SF}_{6}$. Trends of the $t_{1 u}$ vibrational-mode frequencies in octahedral halogen hexafluoride molecules suggest significant vibronic interaction between the $\mathrm{a}_{1 \mathrm{~g}}$ and $\mathrm{t}_{1 \mathrm{u}}$ molecular orbitals in the ground state [34].

\section{V.E. Sulfur $\mathbf{L}_{2,3}$ thresholds: Rydberg series}

\section{Analysis using the Rydberg formula}

Dipole selection rules allow transitions to the s-and d-series Rydberg states below both the sulfur $L_{2}$ and $L_{3}$ edges. Basing the assignment on the sulfur atomic quantum numbers indicates that the $s$-series should begin with $n=4$ and the $d$ series with $n=3$. A least-squares fit of the spectrum of Fig. V-4, using the Rydberg formula, determined the best values for the quantum defects and vibrational spacings, and the energies of the absorption thresholds. Fit results for intensities and widths are most reliable for the more intense and well-isolated features. Derived values are also given for some of the weaker features, but they should not be regarded as definitive. The fit required the use of many adjustable parameters, and regions of overlapping peaks can probably be fitted nearly as well with other combinations of intensities and widths. The background was modeled by the sum of a linear contribution, the tail of two Voigt functions from peaks 3 and 4 (see Fig. V-2), and several overlapping Gaussian peaks to represent the $(S 2 p)^{-1} t_{1 u}$ transitions. Two additional broad peaks ( $\mathrm{D}$ and $\mathrm{K}$ ) were necessary to obtain reasonable results. These

may be regarded as essential modifications to the approximated lineshape of the (S $2 p)^{-1} t_{1 u}$ resonances.

The results of the least-squares fit are shown in Fig. V-4 and in Table V-2, and the derived term values are compared to other measurements and calculated values in 
Table V-4. The peak assignments generally agree with those of previous reports based on spectra with fewer discernible features $[5,8,9]$. Even the previously unobserved features are easily assigned (e.g. peak a, the shoulder at $180.7 \mathrm{eV}$, is the (S 2p1/2)-1 6s state). A single value of the quantum defect is sufficient for each series beyond the lowest energy peak. The energy of the (S $\left.2 \mathrm{p}_{3 / 2}\right)^{-1} 4 \mathrm{~s}$ state (peak $\mathrm{A}$ ) is perturbed by $-37 \mathrm{meV}$ and that of

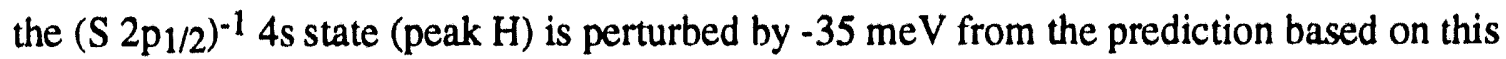
quantum defect. The perturbations of the (S 2p) $)^{-1} 3 \mathrm{~d}$ states are discussed below (see Sec. V.E.2). Note that there has been some uncertainty in the past whether the Rydberg d-series would be observed in this spectrum, because the $\mathrm{t}_{\mathrm{g}}$ and $\mathrm{e}_{\mathrm{g}}$ above-edge resonances might be associated with the atomic sulfur $3 \mathrm{~d}$ orbitals. The present results clearly indicate the presence of the d-series.

The values of the quantum defect obtained from the fit, $\delta_{s}=1.80$ and $\delta_{d}=-0.03$, are somewhat lower than expected for sulfur-based Rydberg series (see Table VI-3 and Section VI.E.4.b). It is also unexpected, based upon atomic spectroscopy, that the s-series is more intense than the $d$-series, for excitation of a p electron. For example, predicted cross section ratios for the hydrogen atom are $I(2 p \rightarrow 4 s) / I(2 p \rightarrow 4 d)=0.25$ [35], compared to 7.6 observed here. Multiple-scattering (MS) calculations for $\mathrm{SF}_{6}$ [32], discussed in more detail in Section V.F, predict that the s-series is more intense than the d-series. The calculated ratio is $I(2 p \rightarrow 4 s) /(2 p \rightarrow 4 d)=22.1$, which is possibly so large because the relative intensity of the $4 \mathrm{~s}$ transition is overestimated in the MS calculation. Better agreement is seen for the ratio $\mathrm{I}(2 \mathrm{p} \rightarrow 5 \mathrm{~s}) / \mathrm{I}(2 \mathrm{p} \rightarrow 4 \mathrm{~d})$, with an observed value of 0.9 and a MS prediction of 1.2 [32], compared to an atomic hydrogen prediction of 0.098 [35].

Both the reduced quantum defects and the inverted s- and d-intensity ratios may be explained by the distinctly non-atomic double-well potential in $\mathrm{SF}_{6}$. The potential barrier excludes the outer-well Rydberg states from the interior of the molecule, leading to better screening of the nuclear charge and thus smaller quantum defects. The intense transitions to $d$-derived $t_{2 g}$ and $e_{g}$ inner-well states above threshold can steal intensity from the below- 
threshold d-Rydberg series. Of course, the below-threshold s-derived a $1 \mathrm{~g}$ inner-well resonance similarly steal intensity from the s-Rydberg series, but the $\mathrm{a}_{1 \mathrm{~g}}$ resonance is much less intense than the $t_{2 g}$ and $e_{g}$ resonances (see Table V-1), and so should steal less. The net effect of these intensity redistributions is to reduce the d-series intensity, relative to the $s$-series. Further calculations of the energies and intensities of these transitions would be helpful to better understand the origin of these unusual results.

Despite these anomalies, the Rydberg formula is remarkably successful at predicting the energies of the higher Rydberg states. Such simplicity in a polyatomic molecule, with 70 electrons, may be a result of the small size of the molecule; the groundstate $S-F$ bond length is only $1.561 \AA$, while the smallest Rydberg orbital, $4 \mathrm{~s}$, has an estimated mean radius of $\approx 3.8 \AA$ [36]. For the $3 \mathrm{~d}$ and $5 \mathrm{~s}$ Rydberg orbitals, the estimated mean radii are $=5.6 \AA$ and $\approx 8.0 \AA$, respectively. This shows that even the lowest Rydberg orbitals lie mainly in the outer well of Fig. V-1. Further evidence for the extra-molecular nature of these Rydberg states comes from a recent photoabsorption experiment [7]. That work showed that the sulfur $L_{2,3}$ Rydberg resonances observed, as here, for gas-phase $\mathrm{SF}_{6}$, are absent in the corresponding spectrum of condensed $\mathrm{SF}_{6}$.

\section{Multiplet splitting}

The octahedral symmetry of the $\mathrm{SF}_{6}$ molecule induces multiplet splitting of each sulfur atomic $d$ orbital into $t_{2 g}$ and $e_{g}$ molecular orbitals. A naive model would then predict four transitions in the sulfur $L_{2,3}$ spectrum corresponding to a single nd orbital, arising from the combination of two core holes ( $2 \mathrm{p}_{1 / 2}$ and $2 \mathrm{p}_{3 / 2}$ ) with two levels (nd- $\mathrm{t}_{2}$ and nd-

$e_{g}$ ). A more complete analysis shows, however, that the ( $\left.2 p\right)^{-1}$ nd configuration leads to seven dipole-allowed transitions [37]. These "extra" transitions essentially result from a full consideration of spin-orbit splitting in the nd orbital and electron-hole coupling (see Section VI.E.1 for a more detailed discussion). In the present case, however, where the 
multiplet splitting is small and is only resolved in regions of overlapping peaks, the naive model given above should be an adequate description.

Core-excited Rydberg states show multiplet splitting in many molecular systems [38 39,40]. Generally, this splitting is expected to be largest for the lowest states of a given Rydberg series because these states feel the strongest anisotropic molecular field. In the d-symmetry Rydberg series observed here, multiplet splitting is resolved only for the (S $2 p)^{-1} 3 d$ states. Fits of the lineshape near the expected energy of the (S 2p3/2)-13d state reveal the presence of two shoulders at 178.80 and $178.85 \mathrm{eV}$ just above the main peak at 178.76. The most likely assignment is that the peak at 178.76 is the $(\mathrm{S} 2 \mathrm{p} 3 / 2)^{-1} 3 \mathrm{~d}-\mathrm{t}_{2 \mathrm{~g}}$ state, the first shoulder is the $\left(S 2 p_{3 / 2}\right)^{-1} 3 \mathrm{~d}-\mathrm{e}_{\mathrm{g}}$ state, and the higher shoulder is a vibrational sideband associated with the (S 2p3/2)-1 3d-t2g state. This results in a multiplet splitting of $39 \mathrm{meV}$ and a vibrational spacing of $84 \mathrm{meV}$. The weighted average of the energies of the $\left(S 2 p_{3 / 2}\right)^{-1} 3 d-t_{2 g}$ and $-e_{g}$ states is perturbed by $-24 \mathrm{meV}$ from the prediction based on the quantum defect given above.

Analysis of the lineshape around the expected energy of the $\left(\mathrm{S}_{2} \mathrm{p}_{1 / 2}\right)^{-1} 3 \mathrm{~d}$ state is complicated by the overlapping Rydberg states leading up to the sulfur $L_{3}$ edge at 180.27 $\mathrm{eV}$. The fit shows, however, that there is more intensity in the region $179.95-180.05 \mathrm{eV}$ than would be expected from only the $\left(\mathrm{S} 2 \mathrm{p}_{3} / 2\right)^{-1} 7 \mathrm{~d}$ state at $180.00 \mathrm{eV}$. This result is consistent with the presence of the $\left(\mathrm{S}_{2} \mathrm{p}_{1 / 2}\right)^{-1} 3 \mathrm{~d}-\mathrm{e}_{\mathrm{g}}$ state at $180.00 \mathrm{eV}, 39 \mathrm{meV}$ above the $\left(\mathrm{S} 2 \mathrm{p}_{1 / 2}\right)^{-1} 3 \mathrm{~d}-\mathrm{t}_{2 \mathrm{~g}}$ state at $179.96 \mathrm{eV}$. Similarly, the vibrational shoulder expected for the $\left(S 2 p_{1 / 2}\right)^{-1} 3 d-t_{2 g}$ state overlaps the ( $\left.2 p_{3 / 2}\right)^{-1} 8 d$ state, accounting for its anomalously high intensity. The energy of the ( $\left.2 \mathrm{p}_{1 / 2}\right)^{-1} 3 \mathrm{~d}-\mathrm{t}_{2 \mathrm{~g}}$ state is perturbed by $-36 \mathrm{meV}$ from the prediction based on the quantum defect given above. 


\section{Linewidths of the Rydberg resonances}

The linewidths of the Rydberg resonances are significantly smaller than those of the inner-well resonances near the sulfur $L_{2,3}$ thresholds, as can be seen by comparing the values given in Tables V-1 and V-2. Exact linewidths for the individual Rydberg states ar not easily obtained because nearly all the resonances lie in regions of overlapping features. Nevertheless, the fit is good enough in most regions to allow the deconvolution of reliable individual 'inewidths for the more intense resonances. In particular, the extracted total

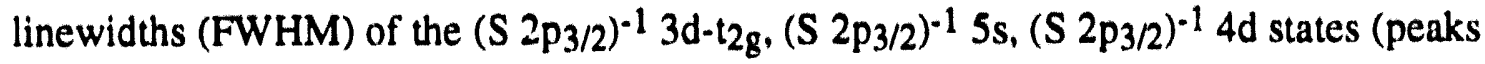
$\mathrm{J}, \mathrm{L}, \mathrm{O}$ ) are 57,54 , and $60 \mathrm{meV}$, respectively. Assuming a Gaussian contribution of 33 $\mathrm{meV}$ from the instrumental resolution, the derived Lorentzian natural linewidths are 38(8), $35(8)$, and $42(8) \mathrm{meV}$, respectively. These results are striking, considering the much larger Lorentzian width (at least $190 \mathrm{meV}$ ) of the energetically nearby inner-well (S 2p1/2,3/2) $)^{-1}$ a $1 \mathrm{~g}$ states (see Section V.D.2).

The large difference in the natural linewidths of the Rydberg resonances and the $(S 2 p)^{-1} a_{1 g}$ resonances can be explained by considering the decay mechanisms of these states. Semi-empirical calculations [41] for atomic sulfur show that a sulfur $2 p$ core hole will decay primarily by an Auger process. The predicted natural linewidth is $54 \mathrm{meV}$. In the case of $\mathrm{SF}_{6}$, the fluorine atoms withdraw electron density from the region of the sulfur core hole [42], resulting in a decrease in the Auger decay rate simply because the valence electrons are further away than in the atom. This qualitatively explains the natural linewidth of $<54 \mathrm{meV}$ for some of the Rydberg resonances observed in the present work, and also predicts a very narrow natural linewidth for photoelectrons emitted from the sulfur $2 p$ subshell in $\mathrm{SF}_{6}$.

In the (S 2p)-1 a $1 \mathrm{~g}$ resonances, the excited electron occupies an orbital which must overlap the S $2 p$ core hole strongly, since the absorption resonance is intense. Apparently the availability of this additional electron, which can dipole-couple to the core hole, 
significantly accelerates the Auger process, resulting in the large natural linewidths observed for these states. This phenomenon emphasizes the substantial difference between the spatial distributions of the inner-well $\mathrm{a}_{\mathrm{g}}$ orbital and the outer-well Rydberg orbitals. Moving a single electron from the inner well to the outer well can increase the lifetime of a core-excited state by a factor of at least five.

If this explanation for the reduced lifetime of the $\left(\mathrm{S}_{2} \mathrm{p}_{1 / 2,3 / 2}\right)^{-1} \mathrm{a}_{1 \mathrm{~g}}$ resonances is correct, then the resonant autoionization spectrum should reflect mainly a "participant" decay process. In other words, the photoelectron spectrum obtained with the photon energy tuned to the resonance should show an enhancement of some valence or subvalence peaks, but not show any additional peaks, as compared to off-resonant excitation. In fact, this experiment has already been performed [43], and the resonance spectrum shows evidence of both participant and spectator decay. Spectator decay refers to autoionization processes not involving the excited $a_{1} g$ electron; these lead to additional peaks in the spectrum, not observed off-resonance. Thus these autoionization results are not entirely consistent with the present linewidth results. Detailed calculations of the (S $2 \mathrm{p})^{-1} \mathrm{a}_{1 \mathrm{~g}}$ resonance decay rates might clarify these uncertainties. In any case, the present results strongly suggest that the participant process plays a role in the decay of the ( $2 p)^{-1}$ a $1 \mathrm{~g}$ resonances. This helps to resolve one question raised in Ref. [43]; specifically, whether the resonant enhancement of the photoelectron peaks at $-40 \mathrm{eV}$ binding energy arises from a spectator-peak satellite or from a simple participant process. The latter interpretation is supported by the present results.

\section{Vibrational structure}

Several of the s- and d-symmetry Rydberg states observed show high-energy shoulders. These may be assigned to final states which include the excitation of a quantum of vibrational energy. The only dipole-allowed vibrational excitation is for the single $a_{1 g}$ 
symmetric stretch mode $\left(v_{1}\right)$. This rule applies to all the s- and d-symmetry excitations. The best example is the (S 2 $\left.\mathrm{p}_{3 / 2}\right)^{-1} 4 \mathrm{~s}$ state (peak $\mathrm{A}$ ) at $177.42 \mathrm{eV}$ and its shoulder at $177.50 \mathrm{eV}$ (peak B). This shoulder is assigned to the vibrational excitation of the $v_{1}$ mode in the (S 2p $\left.p_{3 / 2}\right)^{-1} 4 \mathrm{~s}$ state. A Franck-Condon (FC) analysis [22] of the $\left(S 2 p_{3 / 2}\right)^{-1} 4 \mathrm{~s}$ peak manifold (see Fig. V-7) used harmonic oscillator potentials and Voigt lineshapes. The excited-state parameters obtained were $h v^{\prime}=69 \mathrm{meV}$ and $R^{\prime}=1.58 \AA$ (or $1.54 \AA$ ) compared to $h V^{\prime \prime}=95.9 \mathrm{meV}$ and $\mathrm{R}^{\prime \prime}=1.561 \AA$ in the ground state $[20,21]$. As noted above (in Section V.D.2), in the harmonic oscillator approximation used here, equal contractions and extensions of the bond give equivalent results, so the fit cannot distinguish between the two values given for the S.F bond length $R$ '. However the decrease in the vibrational energy spacing suggests a weakening of the S-F bonds, corresponding to a lengthening of the bond distance. The relative FC factors resulting from the fit are 1.000 : $0.417: 0.032: 0.000$.

The $\left(S 2 p_{1 / 2}\right)^{-1} 4 \mathrm{~s}$ state, peak $\mathrm{H}$ at 178.63 , has the shoulder I at 178.69 . This is a vibrational sideband, analogous to peak B. A FC fit is not practical in this region of overlapping peaks, but the vibrational spacing determined from the least-squares fit of Fig. $\mathrm{V}-4$ is $\mathrm{hV}^{\prime}=61 \mathrm{meV}$, which is close to the value determined for the $\left(S 2 \mathrm{p}_{3 / 2}\right)^{-1} 4 \mathrm{~s}$ state. The derived intensity ratio of peak I to peak $\mathrm{H}$ is $1.00: 0.417$, identical to the corresponding value for the (S 2 $\left.\mathrm{p}_{3 / 2}\right)^{-1} 4 \mathrm{~s}$ state. The similarity of the vibrational parameters of these two states is expected, because the difference in the core-hole. ( $\mathrm{S}$ $\left.2 p_{3 / 2}\right)^{-1}$ vs. (S $\left.2 p_{1 / 2}\right)^{-1}$, should have little influence on the inter-atomic bonds.

The $\left(S 2 p_{3 / 2}\right)^{-1} 5$ s peak $L$ at $178.95 \mathrm{eV}$ shows a vibrational shoulder $M$ at 179.02 $\mathrm{eV}$. The small intensity ratio estimated from the fit $(1.00 ; 0.13)$ suggests that the bond length of the $\left(S 2 p_{3 / 2}\right)^{-1} 5$ s state changes less, relative to the ground state, than that of the (S 2 $\left.\mathrm{p}_{3 / 2}\right)^{-1} 4 \mathrm{~s}$ state. Similarly, the spacing determined from the fit, $h v^{\prime}=74 \mathrm{meV}$, is closer to the ground-state value than the spacing determined above for the (S $\left.2 p_{3 / 2}\right)^{-1}$ is state. These results and the lack of detectable vibrational structure in the higher members of this 
series are expected, because higher Rydberg orbitals have less amplitude within the molecule and are more completely non-bonding. The $\left(\mathrm{S}_{2} \mathrm{p}_{1 / 2}\right)^{-1} 5 \mathrm{~s}$ peak $\mathrm{V}$ at $180.15 \mathrm{eV}$ shows a vibrational shoulder $\mathrm{W}$ at $180.22 \mathrm{eV}$. This feature has not been fit very accurately because it lies in a region where the background is difficult to model.

Vibrational excitations are also observed in several of the d-symmetry Rydberg states. The $\left(S 2 p_{3 / 2}\right)^{-1} 3 d-t_{2} g$ state, as mentioned in Section V.E.2, has a vibrational sideband with a spacing of $h v^{\prime}=85 \mathrm{meV}$ and an intensity ratio of $1.00: 0.12$. This ratio is comparable to that of the nearby $\left(S 2 p_{3 / 2}\right)^{-1} 5 s$ state, while the spacing is slightly larger, indicating that the $3 \mathrm{~d}-12 \mathrm{~g}$ orbital is slightly more non-bonding than the $5 \mathrm{~s}$ orbital. These parameters cannot be derived for the $\left(S 2 p_{1} / 2\right)^{-1} 3 d-12 g$ state, where a vibrational shoulder may be present, but is obscured by the overlapping (S 2p3/2) $)^{-1} 8 \mathrm{~d}$ state. The $\left(S 2 p_{3} / 2\right)^{-1} 4 d$ state, peak $\mathrm{O}$ at $179.44 \mathrm{eV}$, shows a weak vibrational shoulder $\mathrm{P}$ at $179.54 \mathrm{eV}$. The corresponding (S 2p1/2) $)^{-1} 4 \mathrm{~d}$ state, peak $\mathrm{Z}$ at $180.64 \mathrm{eV}$, does not show a vibrational shoulder. Peak P might therefore be alternatively assigned as a second vibrational peak of the $\left(S 2 p_{1 / 2}\right)^{-1} 4 \mathrm{p}$ state, peak $N$ at 179.36 . The $\left(S 2 p_{3 / 2}\right)^{-1} 5 \mathrm{~d}$ peak $(i)$ at $179.74 \mathrm{eV}$ has a vibrational shoulder $R$ at $179.83 \mathrm{eV}$, and the corresponding $\left(S_{2} 2 \mathrm{p}_{1 / 2}\right)^{-1}$ Sit peak b at 180.94 $\mathrm{eV}$ has a similar shoulder $\mathrm{c}$ at $181.03 \mathrm{eV}$. Both of these vibrational excitations have a spacing of $h v^{\prime}=95 \mathrm{meV}$, quite close to the ground-state value $(95.9 \mathrm{meV})$. This reflects the non-bonding nature of these higher Rydberg states, as well as the small influence upon the bonding by the sulfur $2 p$ core hole.

Overall, the observed vibrational structure indicates that the higher Rydherg states induce only small changes in the bond length and the $v_{1}$ vibrational frequency. Apparently the creation of the sulfur $2 p$ core does not induce notable changes in the S-F bond strength. This is somewhat surprising, considering the polarization of the bonds towards the $F$ atoms in $\mathrm{SF}_{6}$ [42]. As discussed in Sections MI.C.3, III.D.2, and III.E for CO, a core hole is expected to redistribute polarized bonding electrons, leading to a change in the bond strength. Perhaps, in the case of $\mathrm{SF}_{6}$, this effect is not as noticeable because it is 
distributed among six bonds. With moderate advances, high-resolution photoelectron spectroscopy may be able to better study the influence of the sulfur $2 p$ core hole on the structure of $\mathrm{SF}_{6}$. The present results clearly indicate some antibonding character in the lower Rydberg orbitals, particularly in the $4 s$ orbital, suggesting that this orbital has some valence, i.e. inner-well, character. This is consistent with the rather large (S 2p) $)^{-1} 4 \mathrm{~s}$ intensities, as compared to the higher s-states, and the deviation of the $(S 2 p)^{-1} 4 \mathrm{~s}$ energies from predictions of the quantum defect $\delta_{\mathrm{s}}$ which applies to the higher s-states. A calculation which included Configuration Interaction $(\mathrm{Cl})$ also indicated that the $4 \mathrm{~s}$ orbital has some valence character [44].

\section{Sulfur p Rydberg orbitals}

Peak $E$ at $178.23 \mathrm{eV}$ has been assigned to the $\left(\mathrm{S} 2 \mathrm{p}_{3 / 2}\right)^{-1} 4 \mathrm{p}$ state for reasons discussed above (see Section V.D.3). In this assignment, this state sits atop a relatively intense, though broad, $\left(S 2 \mathrm{p}_{1 / 2}\right)^{-1} 1_{1}$ peak centered at $\sim 177.5 \mathrm{eV}$. These excited states share the same symmetry and similar energies, so non-vibronic mixing is likely to occur. Essentially, the $(S 2 p 3 / 2)^{-1} 4 p$ state acquires some inner-well character from this mixing. In contrast, the $\left(S 2 p_{1 / 2}\right)^{-1} 4 p$ state will not be perturbed as strongly by the $\left(S 2 p_{1 / 2}\right)^{-1} t_{14}$ state(s) because the energy separation is larger. This may be seen directly in the level diagram of Fig. V-6, where the $\left(S 2 p_{3 / 2}\right)^{-1} 4 p$ state overlaps the $\left(S_{2} p_{1 / 2}\right)^{-1} t_{1}$ shaded region, while the $\left(S 2 p_{1 / 2}\right)^{-1} 4 \mathrm{p}$ state is $-1.0 \mathrm{eV}$ above the top of this region. To facilitate this comparison, the spin-orbit splitting of the $\left(S_{2} 2 p_{1 / 2,3 / 2}\right)^{-1} t_{1}$ resonances is shown in the diagram, although it was not resolved in the spectra. The $\left(S 2 p_{3 / 2}\right)^{-1} 4 \mathrm{p}$ state may be shifted to a higher energy by the interaction with the $\left(S 2 p_{1 / 2}\right)^{-1} 1_{1}$ state(s), reducing the observed spin-orbit splitting of the $\left(S_{2} 2 p_{1 / 2,3 / 2}\right)^{-1} 4 p$ pair. The $\left(S_{2} p_{1 / 2}\right)^{-1} 4 p$ state can thus be assigned to the weak feature $\mathrm{K}$ at $179.3 \mathrm{eV}$. This assumes a shift of $\approx 0.1 \mathrm{eV}$ of the ( $\mathrm{S}$ $2 p 3 / 2)^{-1} 4 p$ state to higher energy. This is a tentative assignment, but it is the most plausible 
one. Peaks $\mathrm{X}$ and $\mathrm{Y}$ at $180.42 \mathrm{eV}$ and $180.50 \mathrm{eV}$ have been assigned to the $\left(\mathrm{S} 2 \mathrm{p}_{1 / 2}\right)^{-1} \mathrm{5p}$ state and a vibrational sideband, respectively. These are very weak resonances, and their assignment is not certain. A corresponding ( $\mathrm{S} 2 \mathrm{p} 3 / 2)^{-1} 5 \mathrm{p}$ state may be present at 179.22 $\mathrm{eV}$, but only barely above the noise level.

Note that the vibronically-coupled $\left(S 2 p_{3} / 2\right)^{-1} 4 p$ transition (peak E) must already include the excitation of at least one quantum of an anti-symmetric vibrational mode of the excited state. Assuming the offset of the transition energy from the fundamental to be $\mathbf{7 6 . 2}$ $\mathrm{meV}$, the corresponding quantum defect derived from the energy of peak $E$ is $\delta_{\mathrm{p}}=1.48$. This value is not unique; it depends on the vibrational offset, which was rather arhitrarily chosen here to equal the energy spacing of the ground-state $v_{4}$ mode ( $t_{1 u}$ symmetry). Use of the ground-state energies [21] of the other two anti-symmetric modes, $v_{3}(117.5 \mathrm{meV}$, $t_{1 u}$ symmetry) and $v_{6}\left(42.9 \mathrm{meV}, t_{2 u}\right.$ symmetry), would result in $\delta_{p}=1.50$ and 1.46 , respectively. This analysis assumes that the Jahn-Teller effect is small for this electronic state. If the Jahn-Teller effect is large, which is possible, the offset of peak $E$ from the fundamental (S 2p3/2) $)^{-1} 4 p$ electronic transition will also reflect the Jahn-Teller splitting of the vibrational states.

Peak E shows two shoulders F.G at higher energies. These are assigned to transitions to $\left(\mathrm{S} 2 \mathrm{p}_{3} / 2\right)^{-1} 4 \mathrm{p}$ states with additional vibrational excitations. The spacings of the shoulders from the main line determined from a least-squares fit are $116 \mathrm{meV}$ and 220 $\mathrm{meV}$. As in the case of the $(\mathrm{S} 2 \mathrm{p} 3 / 2)^{-1} 4 \mathrm{~s}$ state, excitation of the $\mathrm{a}_{1 \mathrm{~g}}$ vibrational mode is allowed. Vibrational excitations are also allowed of any combination of anti-symmetric modes with an odd number of total quanta, e.g. $2 v_{3}+1 v_{4}$. This odd number includes the single quantum of an anti-symmetric vibration which contributes to the energy of Peak $E$. If the Jahn-Teller effect is small in this electronic state, a Ig $_{\mathrm{g}}$ vibrational excitations should dominate. The ground-state energy of this vibration $\left(\mathrm{hv}_{1}{ }^{\prime \prime}=96 \mathrm{meV}\right)$ is roughly comparable to the spacings observed for peaks $F$ and $G$. If the Jahn-Teller effect is large, excitations of the anti-symmetric modes may have significant intensity. Each combination 
of the anti-symmetric vibrational modes will be split into multiple components by the dynamic Jahn-Teller effect. In that case, the complexity of the resulting situation makes any assignment of these vibrational excitations highly speculative.

\section{Energies and edge jumps of the sulfur $L_{2,3}$ thresholds}

The energies of the sulfur $\mathrm{L}_{2,3}$ thresholds are determined by fitting the Rydberg series. The resulting values, given in Table V-2, are in agreement with previous results from photoabsorption $[1,2,3,4,5]$, EELS [8,9], and photoelectron spectroscopy [14,15], within the expected accuracy of monochromator calibration. The absolute energies from EELS are probably more accurate than those given here. The fit confirms what is apparent by visual inspection: there is no measurable edge jump at these thresholds. This is actually expected for systems with intense resonances near threshold [45]. The intense $t_{2 g}$ absorption lines just above the thresholds, as well as the other nearby intense absorption features, "borrow" oscillator strength from the background of direct photoexcitation of sulfur $2 p$ electrons into low kinetic-energy states of the continuum.

\section{V.F. Sulfur $\mathbf{L}_{2,3}$ thresholds: Comparison with theory}

There are four main theoretical papers concerning the core-level photoabsorption of SF6. Gianturco, Guidotti, and Lamanna (GGL) used a LCAO-MO approach [31]. Hay used the Improved-Virtual-Orbital (Hartree Fock) method [46]. Wallace employed the Multiple Scattering $X \alpha$ method [32]. Nakamatsu, Mukoyama, and Adachi (NMA) used the discrete variational $X \alpha$ method [30]. In addition, Reynaud, et. al., [44] applied a Configuration Interaction $(\mathrm{CI})$ calculation to $\mathrm{ClF}_{6}$, the core-equivalent molecule to sulfurcore-excited $\mathrm{SF}_{6}$. Overall, the theoretical results give a fairly good qualitative agreement 
with experiment. Table V-4 compares measured and calculated term values for the (S 2p) $)^{-1}$ below-threshold resonances.

The assignments given in this paper are essentially consistent with the theoretical results, with the following notable results and exceptions: (1) Wallace predicts both the sand the $d-R y d b e r g$ series below the sulfur $L_{2,3}$ edges, with term values in good agreement with the present results (see Table V-4). The calculated multiplet splitting of the $3 \mathrm{~d}$ state into $t_{2 \mathrm{~g}}$ and $\mathrm{e}_{\mathrm{g}}$ species is $0.07 \mathrm{eV}$, in rough agreement with the result of Hay $(0.1 \mathrm{eV})$, and much less for higher nd states. The observed $3 \mathrm{~d}$ splitting was $39 \mathrm{meV}$ (see Section V.E.2), in excellent agreement with the $0.04 \mathrm{eV}$ prediction of Reynaud, et. al. Wallace's intensity predictions are discussed above, in Section V.E.1. (2) NMA describe the

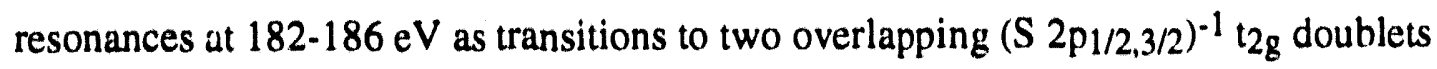
rather than to one doublet. Likewise, the resonance at $196 \mathrm{eV}$ is attributed to two (S $\left.2 \mathrm{p}_{1 / 2,3 / 2}\right)^{-1} \mathrm{eg}_{\mathrm{g}}$ doublets rather than to one doublet. (3) The results of NMA suggest that the potential-barrier model shown in Fig. $\mathrm{V}-1$ is not accurate for $\mathrm{SF}_{6}$. The description of excited states as inner- and outer-well states remains useful, however, to specify the spatial distribution of the excited electron. (4) Hay calculated orbital energies for the (S $2 p)^{-1}$ $4 \mathrm{~s}, 5 \mathrm{~s}, 4 \mathrm{p}$, and $3 \mathrm{~d}$ states that agree closely with those observed (see Table V-4). His predictions for the $(S 2 p)^{-1} a_{1 g}$ and $(S 2 p)^{-1} t_{1 u}$ states are not as accurate. The results in his paper indicate that the (S $2 p)^{-1} t_{1 u}$ state observed here (shaded region in Fig. V-4) is a Rydberg state when it is excited at the more weakly bound thresholds (i.e. valence and subvalence). The state shifts to a larger orbital energy (further below the edge) in the core-hole states. This transition from Rydberg to valence character occurs as the orbital "collapses" into the deepening inner well. This is reflected by an approximate change of +1 in the quantum defect, or, equivalently, a change in the numbering of the Rydberg states (i.e. $5 p$ is relabeled $4 \mathrm{p}$, etc.). The latter representation is used in the present assignment, which labels peak $D$ as the $4 p$ state. Hay refers to the same resonance as the $5 p$ state. (5) Reynaud, et. al., predicted term values for sulfur-core-excited states which are comparable 
to the observed values below the sulfur $L_{2,3}$ edges. In particular, the $3 d-t_{2 g}$ and $3 d-e_{g}$ term values are in excellent agreement with present results.

\section{V.G. Fluorine $\mathrm{K}$ threshold}

Previous reports $[6,8,9]$ of XANES spectra at the fluorine $\mathrm{K}$ edge of $\mathrm{SF}_{6}$ have given assignments similar to those in Table V-3 and Section V.C. The assignments here differ only in the region from $692 \mathrm{eV}$ to $698 \mathrm{eV}$ (see Fig. V-5). The spectrum clearly shows one peak 10 at $694.7 \mathrm{eV}$ with a low-energy shoulder 9 at $692.9 \mathrm{eV}$. Careful lineshape analysis of the spectrum in Fig. V-5, using a Gaussian-broadened arctangent function to model the edge jump, revealed additional intensity in this range (peak 10), as also observed in the spectra of Ref. [9]. The results of the fit are shown in Fig. V-5 and Table V-3. Based on the theoretical results discussed below, the two peaks 9,10 are assigned primarily to two $(\mathrm{F} 1 \mathrm{~s})^{-1} \mathrm{t}_{1 \mathrm{u}}$ final states. The additional peak 11 at $\approx 697 \mathrm{eV}$ has been tentatively assigned to a manifold of weaker $(\mathrm{F} 1 \mathrm{~s})^{-1} \mathrm{t}_{1 \mathrm{u}}$ final states, which are essentially equivalent to (F $1 \mathrm{~s})^{-1} \mathrm{np}$ states. The possibility of contributions from unresolved $(\mathrm{F} 1 \mathrm{~s})^{-1} \mathrm{~ns}$ and nd states cannot be excluded, however. A previous report [8] suggested that the $\approx 1.8 \mathrm{eV}$ splitting of the $694.7 \mathrm{eV}$ peak (into pe:ks 9,10) might be the result of initial-state symmetry splitting of the fluorine $1 \mathrm{~s}$ core level. This is unlikely since no splitting of this level was observed in core-level photoelectron spectra taken with a resolution of $\approx 0.8 \mathrm{eV}$ [47]. Another paper [9] proposed that the splitting of peaks 10 and 11 arises from removal of degeneracy of the $t_{1 u}$ virtual orbital in the reduced symmetry of the $(\mathrm{F} 1 \mathrm{~s})^{-1}$ excited state. That explanation is plausible but apparently has not yet been tested by calculations.

The least-squares fit of the lineshape resulted in large Gaussian contributions to all peaks, indicating non-Lorentzian broadening. No vibrational structure could be detected at a resolution as low as $\approx 310 \mathrm{meV}$. The lineshape of the $(\mathrm{F} 1 \mathrm{~s})^{-1} \mathrm{t}_{2 \mathrm{~g}}$ shape resonance, in 
contrast to the corresponding resonance at the $\mathrm{L}_{2,3}$ thresholds, does not have a large Lorentzian contribution. This non-Lorentzian broadening could be explained by the stronger vibrational excitations expected in a core-excited state with reduced symmetry, as in F-core-excited $\mathrm{SF}_{6}$. An alternative explanation is suggested below.

The four main theoretical papers cited above (in Section V.F) are also qualitatively successful in the assignment of the fluorine $\mathrm{K}$ threshold region, with the following notable results and exceptions. GGL and NMA predict only one strong transition to a $t_{1 u}$ orbital below the fluorine $\mathrm{K}$ threshold. Neither work predicts a resonance of any symmetry to correspond to shoulder 9 at $692.9 \mathrm{eV}$. NMA also predict two weaker (F 1s $)^{-1} \mathrm{t}_{1 \mathrm{u}}$ resonances in the region where extra intensity was observed in this study. Wallace predicts three or four strong and several weak $(\mathrm{F} 1 \mathrm{~s})^{-1} \mathrm{t}_{1 \mathrm{u}}$ resonances in the pre-edge region, with contributions from other resonances being much smaller. GGL assign the resonance just above the fluorine $\mathrm{K}$ edge to a transition to a $t_{1 \mathrm{u}}$ orbital rather than a $\mathrm{t}_{2 \mathrm{~g}}$ orbital. NMA assign approximately one third of the intensity of this resonance to a $(\mathrm{F} 1 \mathrm{~s})^{-1} \mathrm{t}_{1 \mathrm{u}}$ state and the remaining intensity to a slightly lower $(\mathrm{F} 1 \mathrm{~s})^{-1} \mathrm{t} 2 \mathrm{~g}$ state. This is another possible explanation for the difference in lineshapes observed between the $\left(\mathrm{S}_{2} \mathrm{p}_{1 / 2,3 / 2}\right)^{-1} \mathrm{t}_{2 \mathrm{~g}}$ and the $(\mathrm{F} 1 \mathrm{~s})^{-1} \mathrm{t}_{2 \mathrm{~g}}$ states; the resonance above the fluorine $\mathrm{K}$ edge may be broadened by the presence of an offset (F 1s) $)^{-1} \mathrm{t}_{1 \mathrm{u}}$ contribution with possibly a non-Lorentzian lineshape. Hay again had success in predicting the orbital energies of some states below threshold. His results agree very well with the present assignment, with the added possibilities that (1) peak 9 includes contributions from a (F $1 \mathrm{~s})^{-1} \mathrm{a}_{1 \mathrm{~g}}$ state and (2) peak 10 includes contributions from $\left(F_{1 s}\right)^{-1} a_{1 g},(F 1 s)^{-1} e_{g}$, or $(F 1 s)^{-1} t_{2 g}$ states. Overall, Wallace's and Hay's results are in good agreement with the present experimental measurements. Since Hay's work did not predict relative intensities of transitions, Wallace's results were in fact the main guide for the present assignments. 


\section{REFERENCES:}

1. T. M. Zimkina and V. A. Fomichev, Dokl. Akad. Nauk SSSR 169,1304 (1966); Sov. Phys. Dokl. 11, 726 (1967); A. S. Vinogradov and T. M. Zimkina, Opt. Spektroskopiya 32, 33 (1972); Opt. Spectrosc. USSR 31, 364 (1971).

2. M. Nakamura, Y. Morioka, T. Hayaishi, E. Ishigura, and M. Sasanuma, Conference proceedings, Third International Conference on Vacuum UV Radiation Physics, Tokyo (1971) 1, p. A1-6.

3. D. Blechschmidt, R. Haensel, E. E. Koch, U. Nielsen, and T. Sagawa, Chem. Phys. Lett. 14, 33 (1972).

4. B. M. Addison, K. H. Tan, G. M. Bancroft, and F. Cerrina, Chem. Phys. Lett. 129, 468 (1986).

5. E. S. Gluskin, A. A. Krasnoperova, and L. N. Mazalov, Zh. Strukt. Khim. 18, 185 (1977); J. Struct. Chem. USSR 18, 156 (1977).

6. T. M. Zimkina and A. S. Vinogradov, J. Phys. (Paris) C4, 3 (1971); R. E. LaVilla, J. Chem. Phys. 57, 899 (1972).

7. J. S. Tse and Z. F. Liu, Phys. Rev. A 44, 7838 (1991).

8. A. P. Hitchcock and C. E. Brion, Chem. Phys. 33, 55 (1978).

9. K. H. Sze and C. E. Brion, Chem. Phys. 140, 439 (1990).

10. J. L. Dehmer, J. Chem. Phys. 56, 4496 (1972).

11. W. H. E. Schwarz, Angew. Chem. Int. Ed. Engl. 13, 454 (1974); V. I. Nefedov, Zh. Strukt. Khim. 11, 292 (1970); J. Struct. Chem. USSR 11, 272 (1970).

12. T. A. Ferrett, D. W. Lindle, P. A. Heimann, M. N. Piancastelli, P. H. Kobrin, H. G. Kerkhoff, U. Becker, W. D. Brewer, and D. A. Shirley, J. Chem. Phys. 89, 4726 (1988).

13. J. L. Dehmer, A. C. Parr, S. Wallace, and D. Dill, Phys. Rev. A 26, 3283 (1982).

14. K. Siegbahn, C. Nordling, G. Johansson, J. Hedman, P. F. Hedén, K. Hamrin, U. Gelius, and T. Bergmark, ESCA Applied to Free Molecules (North-Holland, Amsterdam, 1969), pp. 94-98.

15. U. Gelius, J. Electron Spectrosc. Relat. Phenom. 5, 985 (1974).

16. R. V. Vedrinskii, A. P. Kovtun, V. V. Kolesnikov, Y. F. Migal, E. V. Polozhentsev, and V. P. Sachenko, Izv. Akad. Nauk SSSR Ser. Fiz. 38, 434 (1974); Bull. Acad. Sci. USSR Ser. Phys. 38, Nr. 3, 8 (1974).

17. U. Nielson, R. Haensel, and W. H. E. Schwarz, J. Chem. Phys. 61, 3581 (1974). 
18. K. H. Sze, C. E. Brion, X. M. Tong, and J. M. Li, Chem. Phys. 115, 433 (1987); N. A. Shklyaeva, L. N. Mazalov, and V. V. Murakhtanov, J. Struct. Chem. USSR 20, 621 (1979).

19. A. R. Boate, J. R. Morton, and K. F. Preston, J. Phys. Chem. 80, 2954 (1976).

20. L. S. Bartell and S. K. Doun, J. Mol. Struct. 43, 245 (1978); V. C. Ewing and L. E. Sutton, Trans. Faraday Soc. 59, 1241 (1963).

21. Y. M. Bosworth, R. J. H. Clark, and D. M. Rippon, J. Mol. Spectrosc. 46, 240 (1973); R. S. McDowell, J. P. Aldridge, and R. F. Holland, J. Phys. Chem. 80, 1203 (1976).

22. See Section I.D.4 of this dissertation.

23. M. Domke, T. Mandel, A. Puschmann, C. Xue, D. A. Shirley, G. Kaindl, H. Petersen, and P. Kuske, Rev. Sci. Instrum. 63, 80 (1992).

24. J. L. Dehmer, A. C. Parr, and S. H. Southworth, in Handbook on Synchrotron Radiation, edited by G.V. Marr (North-Holland, Amsterdam, 1987), Vol. II, pp. 280284.

25. A. P. Hitchcock, C. E. Brion, and M. J. Van der Wiel, J Phys. B: Atom. Molec. Phys. 11, 3245 (1978).

26. C. M. Truesdale, D. W. Lindle, P. H. Kobrin, U. E. Becker, H. G. Kerkhoif, P. A. Heimann, T. A. Ferrett, and D. A. Shirley, J. Chem. Phys. 80, 2319 (1984).

27. G. Herzberg, Molecular Spectra and Molecular Structure (Van Nostrand Reinhold, New York, 1966), Vol. III, pp. 137-41.

28. G. C. King and F. H. Read, Atomic Inner-Shell Physics, edited by B. Crasemann (Plenum, New York, 1985), pp. 357-365.

29. J. F. Ying, C. P. Mathers, and X. T. Leung, Phys. Rev. A 47, R5 (1993).

30. H. Nakamatsu, T. Muknyama, and H. Adachi, J. Chem Phys. 95, 3167 (1991); Chem Phys. 143, 221 (1990).

31.F. A. Gianturco, C. Guidotti, and U. Lamanna, J. Chem. Phys, 57, 840 (1972).

32. R. Scott Wallace, Ph. D. Thesis, Boston University, 1980.

33. V. I. Nefedov and V. A. Fomichev, Zh. Strukt. Khim. 9, 279 (1968); J. Struct. Chem. USSR 9, 217 (1968).

34. A. R. Boate, J. R. Morton, K. F. Preston, and S. J. Strach, J. Chem. Phys, 71, 388 (1979).

35. H. A. Bethe and E. E. Salpeter, Quantum Mechanics of One- and Two-Electron Atoms (Plenum, New York, 1977) p. 265. 
36. Derived using the results for hydrogenic atoms in Ref. [35], p. 17. The principle quantum numbers $n$, used in the formula, were corrected by the respective quantum defects observed here.

37. F. M. F. de Groot, J. C. Fuggle, B. T. Thole, and G. A. Sawatzky, Phys. Rev. B 41, 928 (1990).

38. C. T. Chen, Y. Ma, and F. Sette, Phys, Rev. A40, 6737 (1989); Y. Ma, C. T. Chen, G. Meigs, K. Randall, and F. Sette, Phys. Rev. A44, 1848 (1991).

39. See Sections III.C. 2 and IV.C. 3 of this dissertation.

40. G. R. Wright and C. E. Brion, J. Electron Spectrosc. Relat. Phenom. 4, 25 (1974).

41. M.O. Krause and J. H. Oliver, J. Phys. Chem. Ref. Data 8. 329 (1979).

42. D. P. Santry and G. A. Segal, J. Chem. Phys. 47, 158 (1967); G. L. Bendazzoli, P. Palmieri, B. Cadioli, and U. Pincelli, Molec. Phys. 19, 865 (1970); P. Jeffrey Hay, J. Am. Chem. Soc. 99, 1003 (1977).

43. G. M. Bancroft, K. H. Tan, O.-P. Sairanen, S. Aksela, and H. Aksela, Phys. Rev. A 41, 3716 (1990).

44. C. Reynaud, S. Bodeur, J. L. Maréchal, D. Bazin, P. Millié, I. Nenner, U.

Rockland, and H. Baumgärtel, Chem. Phys. 166, 411 (1992).

45. U. Fano and J. W. Cooper, Rev. Mod. Phys. 40, 441 (1968).

46. P. Jeffrey Hay, J. Am. Chem. Soc. 99, 1013 (1977).

47. Ref. [14], p. 24. 
Table V-1 : $\mathrm{SF}_{6}$ sulfur $2 \mathrm{p}$ inner-well resonances: Assignments and fit results.

\begin{tabular}{llllcc}
\hline Peak & $\begin{array}{c}\text { Energy } \\
(\mathrm{eV})\end{array}$ & \multicolumn{2}{c}{ Assignment } & $\begin{array}{c}\text { Intensity } \\
\text { (arb. units) }\end{array}$ & $\begin{array}{c}\text { Width } \\
\text { (FWHM, eV) }\end{array}$ \\
\hline 1 & 172.27 & $\mathrm{a}_{1 \mathrm{~g}}$ & $(\mathrm{~S} 2 \mathrm{p} 3 / 2)^{-1}$ & 1.00 & 0.87 \\
2 & 173.44 & $\mathrm{a}_{1 \mathrm{~g}}$ & $(\mathrm{~S} 2 \mathrm{p} 1 / 2)^{-1}$ & 1.62 & 1.06 \\
3 & 183.40 & $\mathrm{t}_{\mathrm{g}}$ & $(\mathrm{S} 2 \mathrm{p} 3 / 2)^{-1}$ & 2.71 & 0.76 \\
4 & 184.57 & $\mathrm{t}_{2 \mathrm{~g}}$ & $(\mathrm{~S} 2 \mathrm{p} 1 / 2)^{-1}$ & 5.53 & 0.85 \\
5 & 196.2 & $\mathrm{e}_{\mathrm{g}}$ & $(\mathrm{S} 2 \mathrm{p} 1 / 2,3 / 2)^{-1}$ & 15.7 & 4.1 \\
6 & 205 & multi-electr. excit.? & $\ldots$ & $\ldots$ \\
7 & 209 & multi-electr. excit.? & $\ldots$ & $\ldots$ \\
\hline
\end{tabular}


Table V-2 : $\mathrm{SF}_{6}$ sulfur $2 p$ Rydberg states: Assignments and fit results.

\begin{tabular}{|c|c|c|c|c|c|}
\hline \multirow{2}{*}{$\begin{array}{l}\text { Peak } \\
\text { A } \\
\text { B }\end{array}$} & $\begin{array}{l}\text { Energy } \\
(\mathrm{eV})\end{array}$ & \multicolumn{2}{|c|}{ Assignment } & \multirow{2}{*}{$\begin{array}{l}\text { Intensity } \\
\text { (arb. units) } \\
100.0 \\
41.7\end{array}$} & \multirow{2}{*}{$\begin{array}{c}\begin{array}{c}\text { Width } \\
\text { (FWHM,meV) }\end{array} \\
76 \\
76\end{array}$} \\
\hline & $\begin{array}{l}177.42 \\
177.49\end{array}$ & $4 s$ & $\begin{array}{l}(\mathrm{S} 2 \mathrm{p} 3 / 2)^{-1} \\
\text { vibr. }\end{array}$ & & \\
\hline C & 177.56 & & vibr. & $\ldots$ & ... \\
\hline D & 177.72 & \multicolumn{2}{|c|}{ background } & & 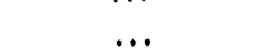 \\
\hline $\mathbf{E}$ & 178.21 & \multicolumn{2}{|c|}{$4 p \quad(S 2 p 3 / 2)^{-1}$} & 19.4 & 102 \\
\hline & 178.33 & & \multirow{3}{*}{$\begin{array}{l}\text { vibr. } \\
\text { vibr. } \\
(\text { (S 2p1/2) }\end{array}$} & $\ldots$ & $\cdots$ \\
\hline G & 178.43 & & & & \\
\hline $\begin{array}{l}\mathrm{H} \\
\mathrm{I}\end{array}$ & $\begin{array}{l}178.63 \\
178.69\end{array}$ & $4 \mathrm{~s}$ & & 54.7 & 85 \\
\hline \multirow[t]{2}{*}{ J } & $/ 178.76$ & \multicolumn{2}{|c|}{$3 d-t_{2 g} \quad\left(S 2 p_{3 / 2}\right)^{-1}$} & 27.2 & 57 \\
\hline & 1178.80 & \multirow{2}{*}{\multicolumn{2}{|c|}{$\begin{array}{ll}3 d-e_{g} & \left(S 2 p_{3 / 2}\right)^{-1} \\
& \text { vibr. }\left(3 d-t_{2 g}\right)\end{array}$}} & $\cdots$ & ... \\
\hline $\mathrm{K}$ & 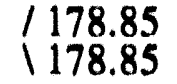 & & & $\cdots$ & ... \\
\hline L & 178.95 & $5 s$ & $\left(\mathrm{~S} 2 \mathrm{p}_{3 / 2}\right)^{-1}$ & 15.4 & 54 \\
\hline $\mathbf{M}$ & 179.02 & & vibr. & $\ldots$ & $\ldots$ \\
\hline $\mathrm{N}$ & 179.36 & $4 p$ & $\left(\mathrm{~S} 2 \mathrm{p}_{1 / 2}\right)^{-1}$ & & \\
\hline 0 & 179.44 & $4 d$ & $\left(\mathrm{~S} 2 \mathrm{p}_{3 / 2}\right)^{-1}$ & 19.1 & 60 \\
\hline $\mathrm{P}$ & $\begin{array}{l}179.50 \\
1179.54\end{array}$ & $6 s$ & $\begin{array}{l}\left(S_{2} 2 p_{3} / 2\right)^{-1} \\
\text { vibr. }(4 d)\end{array}$ & 10.6 & 67 \\
\hline & $/ 179.74$ & $5 d$ & $\left(\mathrm{~S} 2 \mathrm{p}_{3 / 2}\right)^{-1}$ & & \\
\hline Q & 1179.77 & $7 \mathrm{~s}$ & $\left(S 2 p_{3 / 2}\right)^{-1}$ & & \\
\hline $\mathbf{R}$ & 179.83 & & vibr. (5d) & & \\
\hline$S$ & $\begin{array}{l}/ 179.90 \\
\backslash 179.92\end{array}$ & $\begin{array}{l}6 d \\
8 s\end{array}$ & $\begin{array}{l}\left(\mathrm{S} 2 \mathrm{p}_{3 / 2}\right)^{-1} \\
\left(\mathrm{~S} 2 \mathrm{p}_{3 / 2}\right)^{-1}\end{array}$ & & \\
\hline & / 179.96 & $3 d-t_{2 g}$ & $\left(\mathrm{~S} 2 \mathrm{p}_{1 / 2}\right)^{-1}$ & & \\
\hline $\mathrm{T}$ & 180.00 & $7 \mathrm{~d}^{-0}$ & $\left(S 2 p_{3} / 2\right)^{-1}$ & & \\
\hline & $\begin{array}{l}180.00 \\
/ 180.05\end{array}$ & $3 d-e_{g}$ & $\begin{array}{l}\left(S 2 p_{1} 1 / 2\right)^{-1} \\
\text { vibr. }\left(3 d-t_{0}\right)\end{array}$ & & \\
\hline U & $\backslash 180.06$ & $8 d$ & $\left(\mathrm{~S} 2 \mathrm{p}_{3 / 2}\right)^{-1}$ & & \\
\hline V & / 180.13 & $9,10 d$ & $(\mathrm{~S} 2 \mathrm{p} 3 / 2)^{-1}$ & & \\
\hline $\mathrm{W}$ & 1180.15 & 5s & $\left(\mathrm{S} 2 \mathrm{p}_{1 / 2}\right)^{-1}$ & & \\
\hline $\mathrm{L}_{3}$ edge & $\begin{array}{l}180.22 \\
180.27\end{array}$ & & vibr. & & \\
\hline & 180.42 & $5 p$ & $\left(\mathrm{~S} 2 \mathrm{p}_{1 / 2}\right)^{-1}$ & & \\
\hline $\mathrm{Y}$ & 180.50 & & vibr. & & \\
\hline $\mathbf{Z}$ & 180.64 & $4 d$ & $\left(\mathrm{~S} 2 \mathrm{p}_{1 / 2}\right)^{-1}$ & & \\
\hline 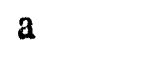 & 180.71 & $6 s$ & $\left(\mathrm{~S} 2 \mathrm{p}_{1 / 2}\right)^{-1}$ & & \\
\hline & / 180.94 & $5 \mathrm{~d}$ & $\left(\mathrm{~S}_{2} \mathrm{p}_{1 / 2}\right)^{-1}$ & & \\
\hline D & $\backslash 180.97$ & $7 s$ & $\left(S 2 p_{1 / 2}\right)^{-1}$ & & \\
\hline c & 181.03 & & vibr. (5d) & & \\
\hline & / 181.10 & $6 d$ & $\left(\mathrm{~S} 2 \mathrm{p}_{1 / 2}\right)^{-1}$ & & \\
\hline$a$ & $\mid 181.12$ & $8 s$ & $(\mathrm{~S} 2 \mathrm{p} 1 / 2)^{-1}$ & & \\
\hline e & 181.20 & $7 d$ & $\left(\mathrm{~S} 2 \mathrm{p}_{1 / 2}\right)^{-1}$ & & \\
\hline & 181.27 & $8 d$ & $\left(\mathrm{~S} 2 \mathrm{p}_{1 / 2}\right)^{-1}$ & & \\
\hline L2 edge & 181.48 & & & & \\
\hline
\end{tabular}


Table V-3 : SF6 fluorine K inner-well resonances: Assignments and fit results.

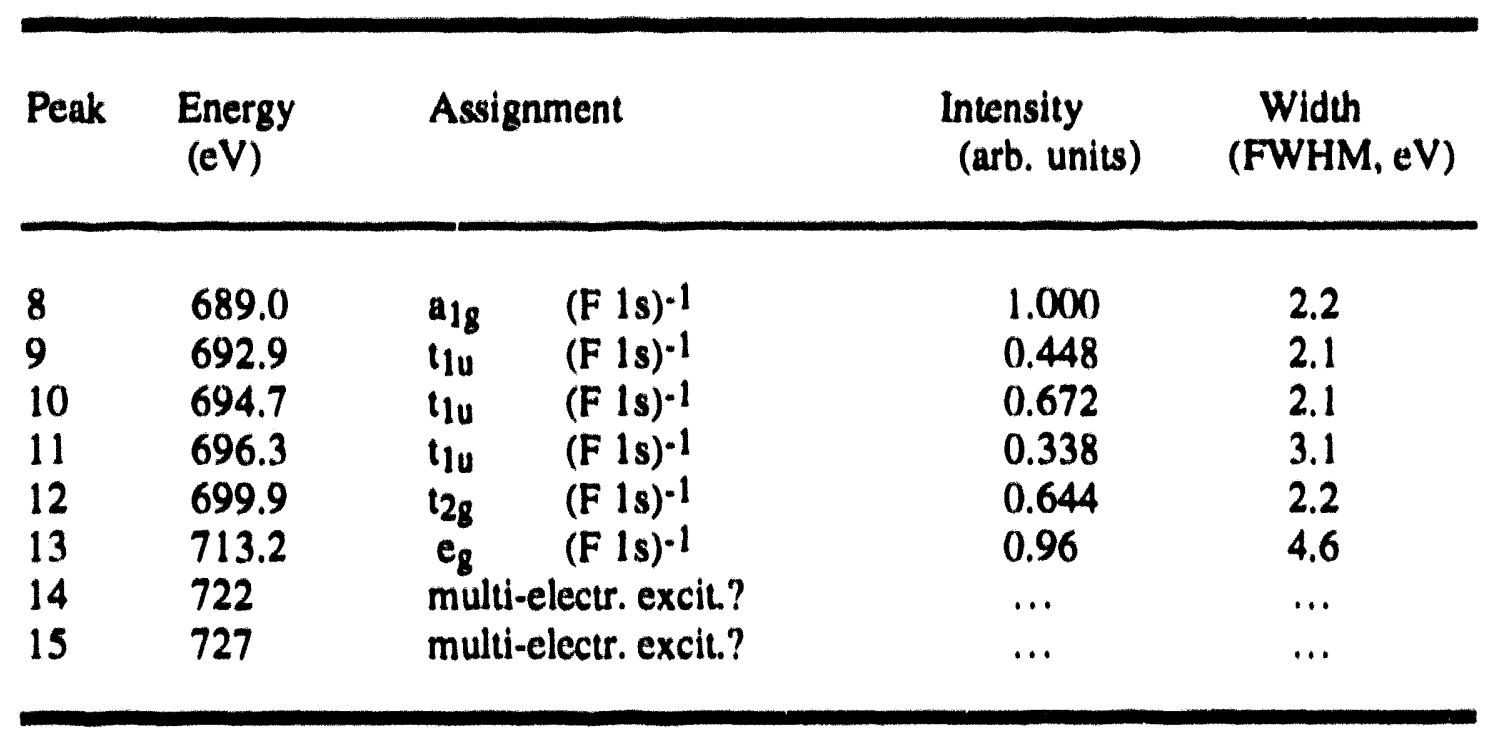


Table V-4: Comparison of measured and calculated term values for the $(S 2 p)^{-1}$ belowedge resonances. All energies are in $\mathrm{eV}$.

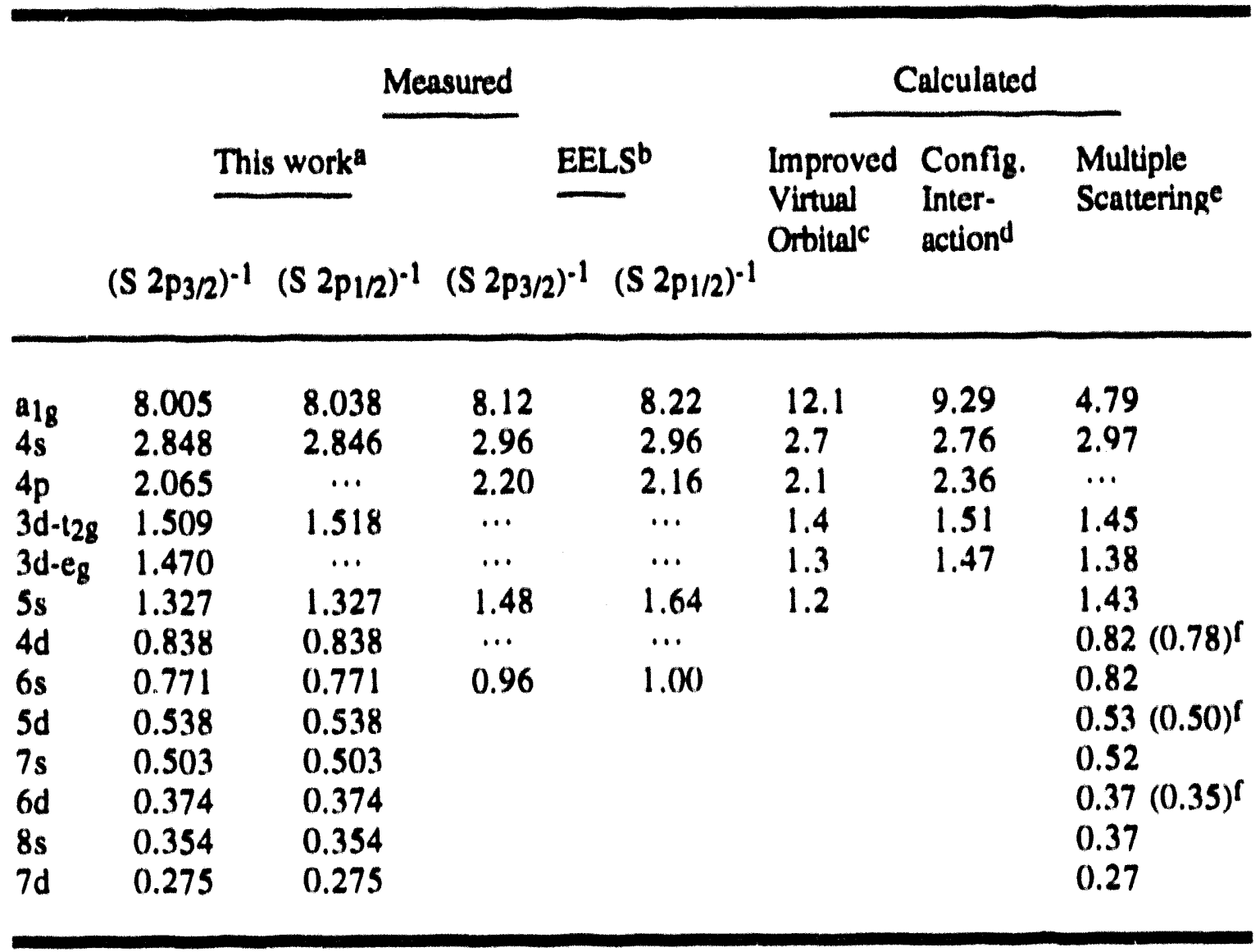

aObtained from fit results for transition energies using threshold values $L_{3}=180.275 \mathrm{eV}$ and $\mathrm{L}_{2}=181.478 \mathrm{eV}$.

bRef. [9]. Note that these term values are based upon an assignment which differs slightly from the present one.

CRef. [44]. Calculated for $\mathrm{ClF}_{6}$, i.e. the core-equivalent molecule to sulfur-core-excited SF6.

dRef. [46]. The value listed here for the $4 p$ level was attributed in Ref. [46] to 5p, due to a difference in notation.

eMeasured from Fig. 39 in Ref. [32].

The two values given refer to the nd- $t_{2 g}$ (nd-e $g$ ) levels. 


\section{FIGURE CAPTIONS:}

Figure V-1. Schematic representation of a double-well potential (not to scale). The barrier divides the potential curve into two regions: the inner well and the outer well. Valence orbitals are located mainly in the inner well. Rydberg orbitals are located mainly in the outer well. Some possible energies of such orbitals are shown as horizontal lines in the figure. Note that, in some inner-well states, the electron has positive energy. Such states are quasi-bound; the electron rapidly tunnels through the barrier and escapes the molecule.

Figure V-2. Photoabsorption spectrum of $\mathrm{SF}_{6}$ near the sulfur $\mathrm{L}_{2.3}$ edges. The numbered peaks are assigned in Table V-1. For peaks 1-4, the line through the data points is a leastsquares fit resulting in the individual components shown. Each component peak is a Voigt function. The results of the fit are given in Table V-1. The data points are connected in the Rydberg region to guide the eye. The inset data showing peaks 6 and 7 in greater detail were measured using the 2442 line/mm grating.

Figure V-3. The $(S 2 p)^{-1} a_{1 g}$ resonances of $S_{6}$. The data here were obtained using the small-source running mode of BESSY, resulting in higher resolution than in Fig. V-2. The line through the data points is the result of a Franck-Condon analysis, with the individual vibronic transitions represented by Voigt functions and plotted as subspectra. Note the larger linewidths of the individual vibronic peaks for the higher-energy $\left(\mathrm{S}_{2} \mathrm{p}_{1 / 2}\right)^{-1} \mathrm{a}_{1 \mathrm{~g}}$ resonance, as compared to the $\left(S 2 p_{3 / 2}\right)^{\cdot 1} \mathrm{a}_{1 \mathrm{~g}}$ components.

Figure V-4. Sulfur $L_{2,3}$ pre-edge fine structure of the SF6 photoabsorption spectrum. The shaded area indicates the contribution of the (S $2 p)^{-1} / 1 u$ transitions to the overall background. The peaks labeled by letters are assigned in Table V-2. The line through the data points is the result of a least-squares fit. For the fit, the background was modeled by 
a linear contribution, the tail of a Voigt function from peak 3 (see Fig. V.2), and several overlapping Gaussian peaks to represent the $(S 2 p)^{-1} t_{1}$ transitions. Each of the individual component peaks shown is a Voigt function. The results of the fit are given in Table V-2. The data in this figure were measured using the 2442 line/mm grating.

Figure V.s. Photoabsorption spectrum of $\mathrm{SF}_{6}$ near the fluorine $\mathrm{K}$ edge. The numbered peaks are assigned in Table V.3. The line through the data points from 680 to $708 \mathrm{eV}$ is a least-squares fit with the individual components shown. Each component peak is a Voigt function. A Gaussian-broadened arctangent function models the edge jump. The results of the fit are given in Table V.3.

Figure V-6. Level diagram of the core-excited states of $S_{6}$ at the sulfur $L_{2,3}$ and fluorine $K$ edges. The relative energies of the states are plotled to scale, using the results given in Tables V-1.2.3. The fluorine $K$ edge is aligned with the weighted average of the sulfur $L_{2,3}$ edges to facilitate the comparison of relative state energies. On the left side of the diagram, only the states with a (S $\left.2 p_{3 / 2}\right)^{-1}$ core hole are labeled. The corresponding states with a $\left(S 2 p_{1 / 2}\right)^{-1}$ core hole are shown as a separate series, hut not labeled. Several Rydberg states are shown below the sulfur $L_{2,3}$ edges, but only the two lowest states are labeled. The spin-orbit splitting of the $\left(S 2 p_{1 / 2,3 / 2}\right)^{-1} \mathrm{e}_{\mathrm{g}}$ doublet was not resolved in the spectrum and the short solid horizontal line shows the energy of the single feature observed. The energies of these two individual states have been approximated by using the spin-orbit splitting and peak intensity ratio measured for the (S 2p1/2,3/2) $)^{-1}{ }_{2 g}$ doublet. The levels obtained are shown by the horizontal dashed lines. The shaded regions indicate the positions of broad features in the spectra which may include contributions from more than one state. In the case of the (S 2p1/2,3/2) $)^{-1}$ //u shaded regions, the spin-orbit splitting shown was not actually resolved in the spectra, and, in addition, there may be two separate 
resonances. The broad shaded region at the fluorine $K$ edge is tentatively assigned to an unresolved manifold of $(\mathrm{F} / \mathrm{s})^{-1}$ t/u states. See text for further details.

Figure V-7. Franck Condon analysis of the vibrational structure of the $(S 2 p)^{-1} 48$ state (peaks A,B,C in Fig. V-4). The ground-state parameters were taken from Refs. [20] and [21] and the final-state parameters were varied to obtain the best fit. Each of the individual component peaks shown is a Voigt function. See text for further details. 


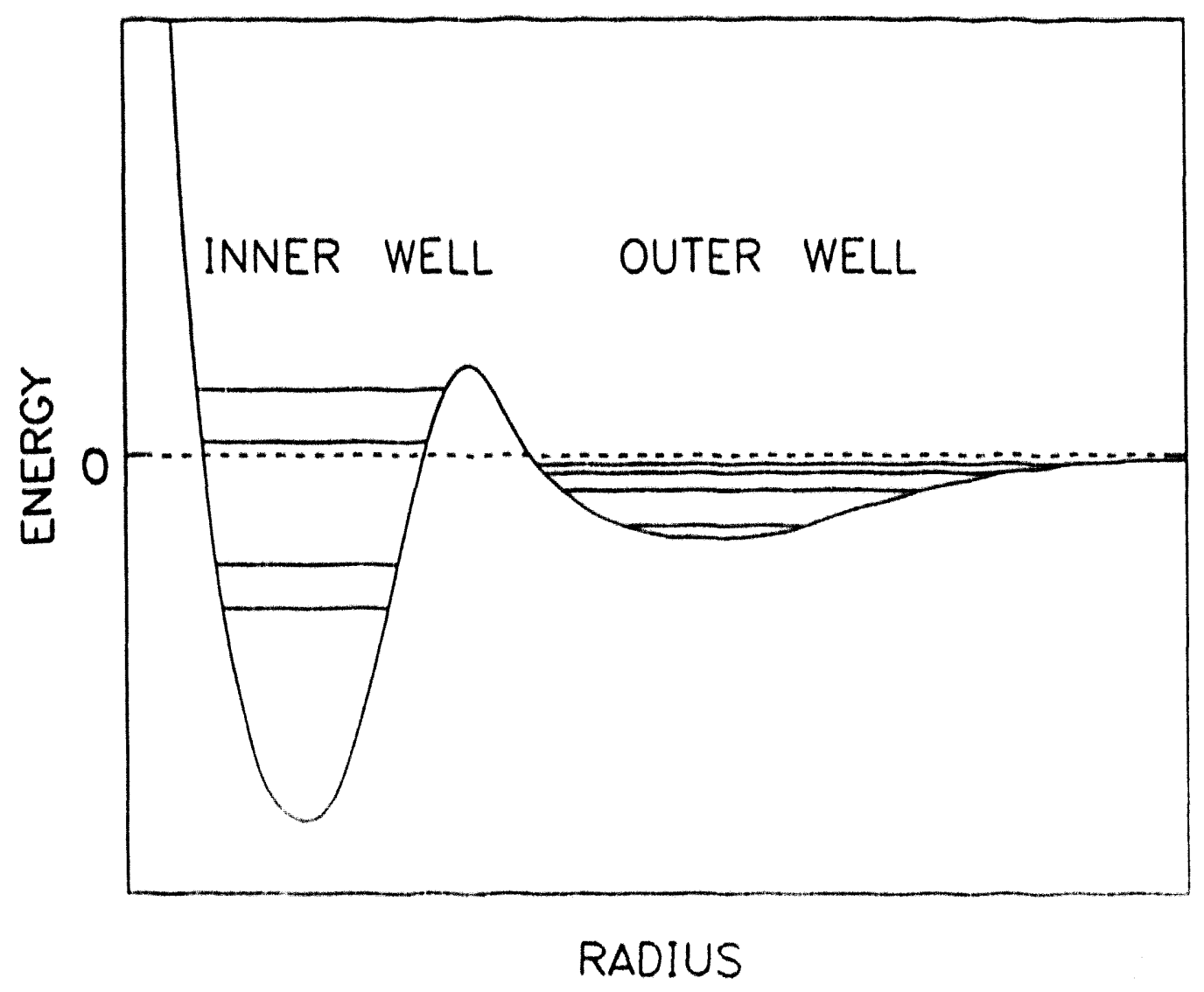

Figure V-1 


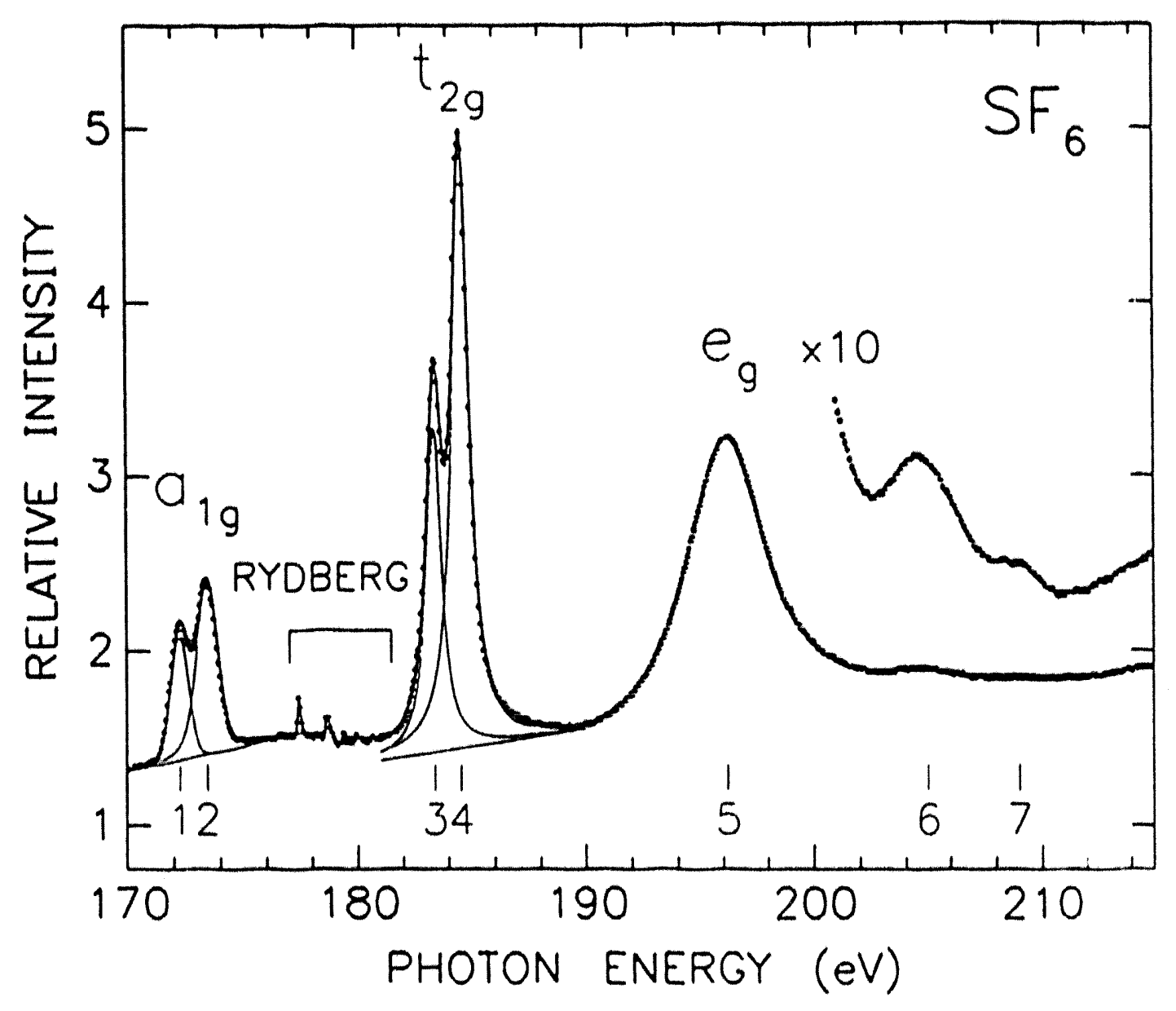

Figure V-2 


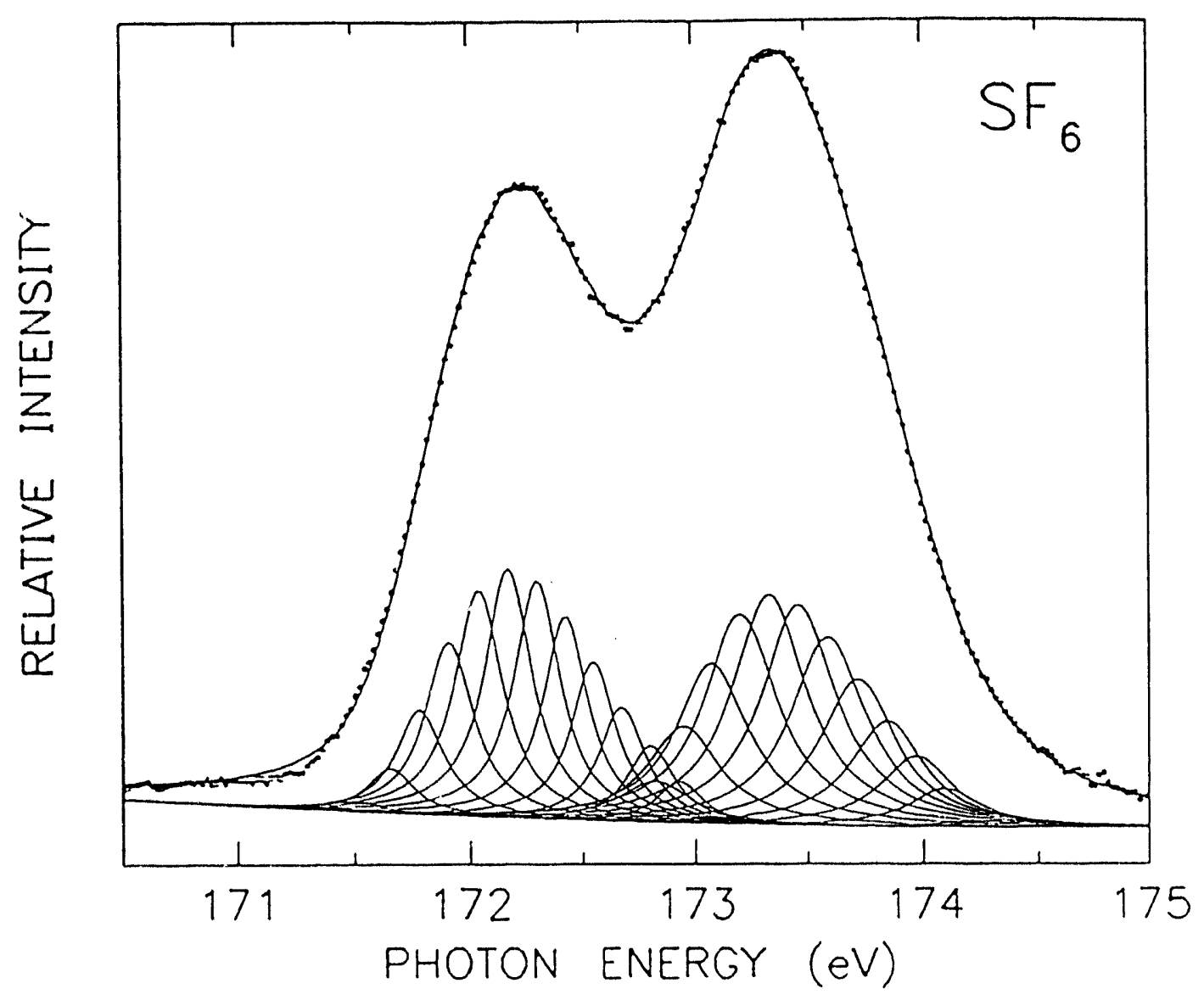

Figure V-3 


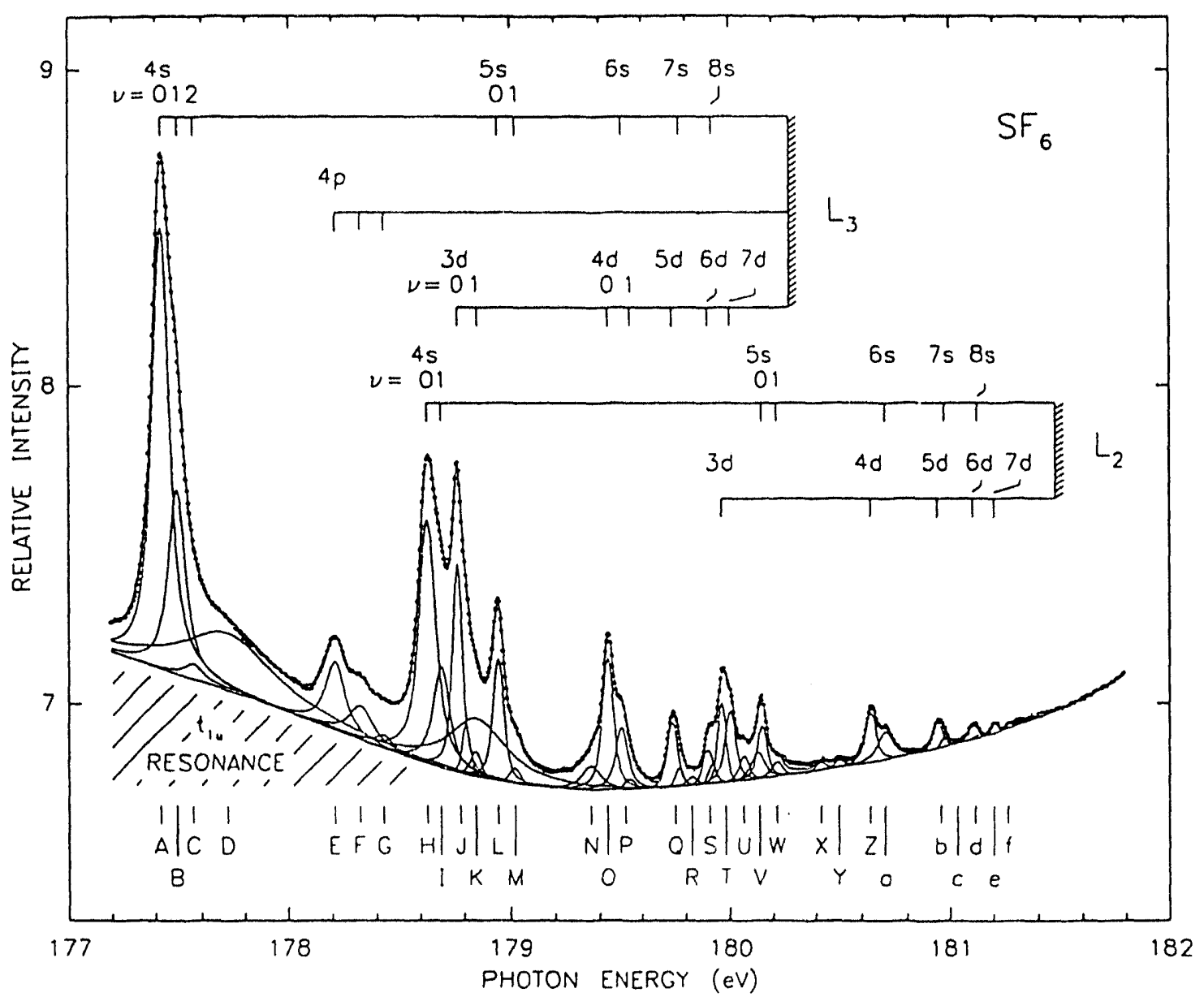

Figure V-4 


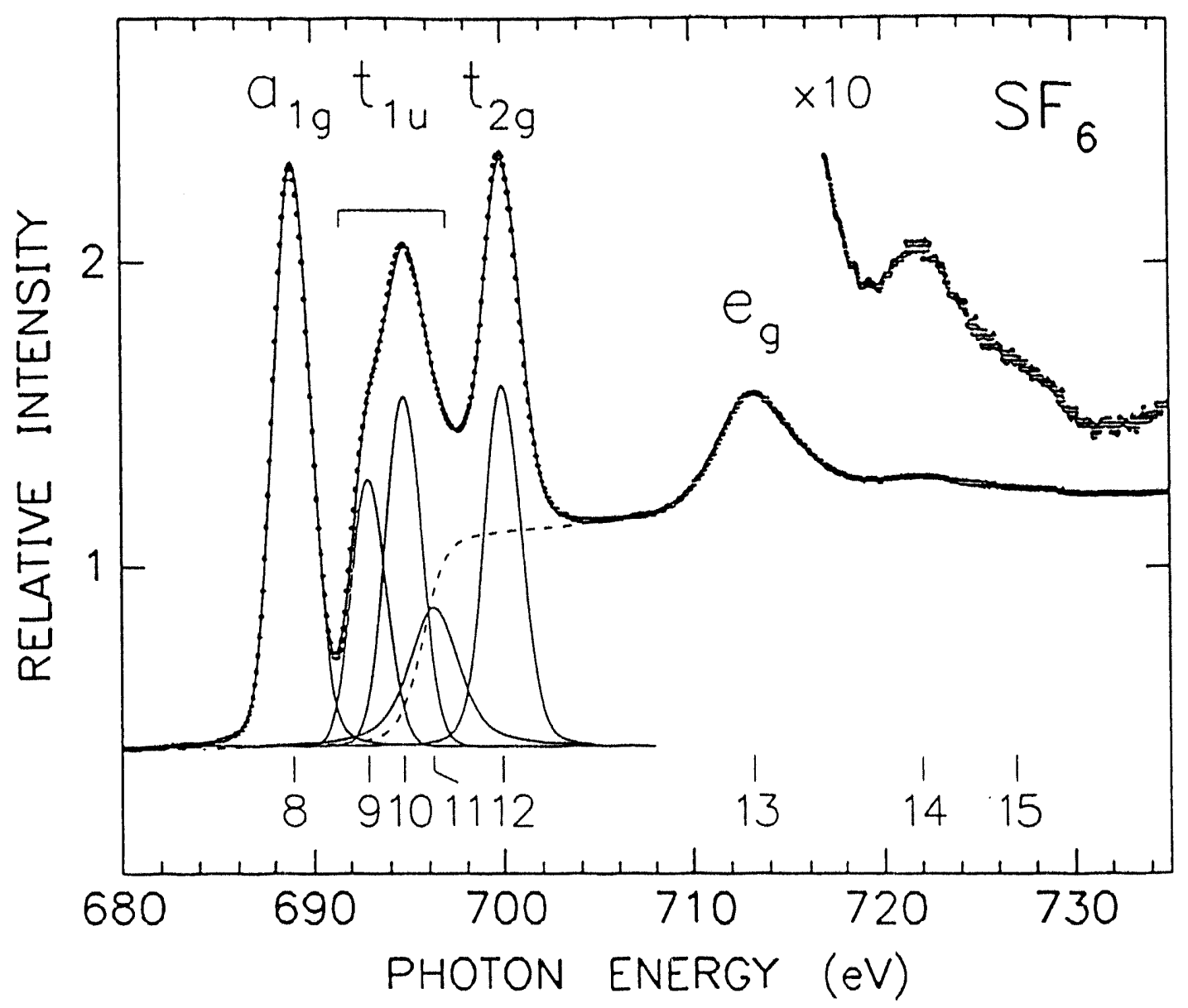

Figure V-5 


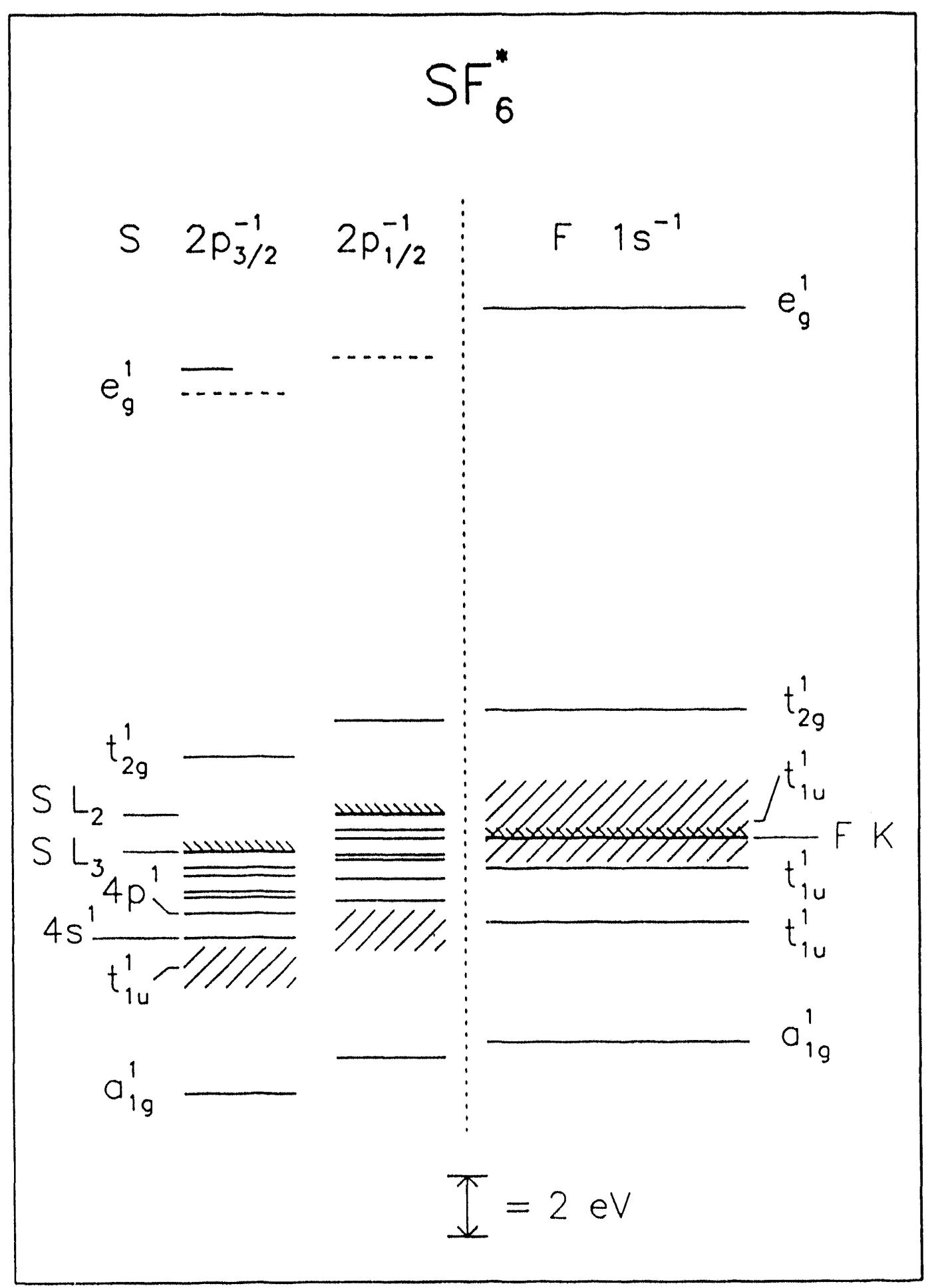

Figure V-6 


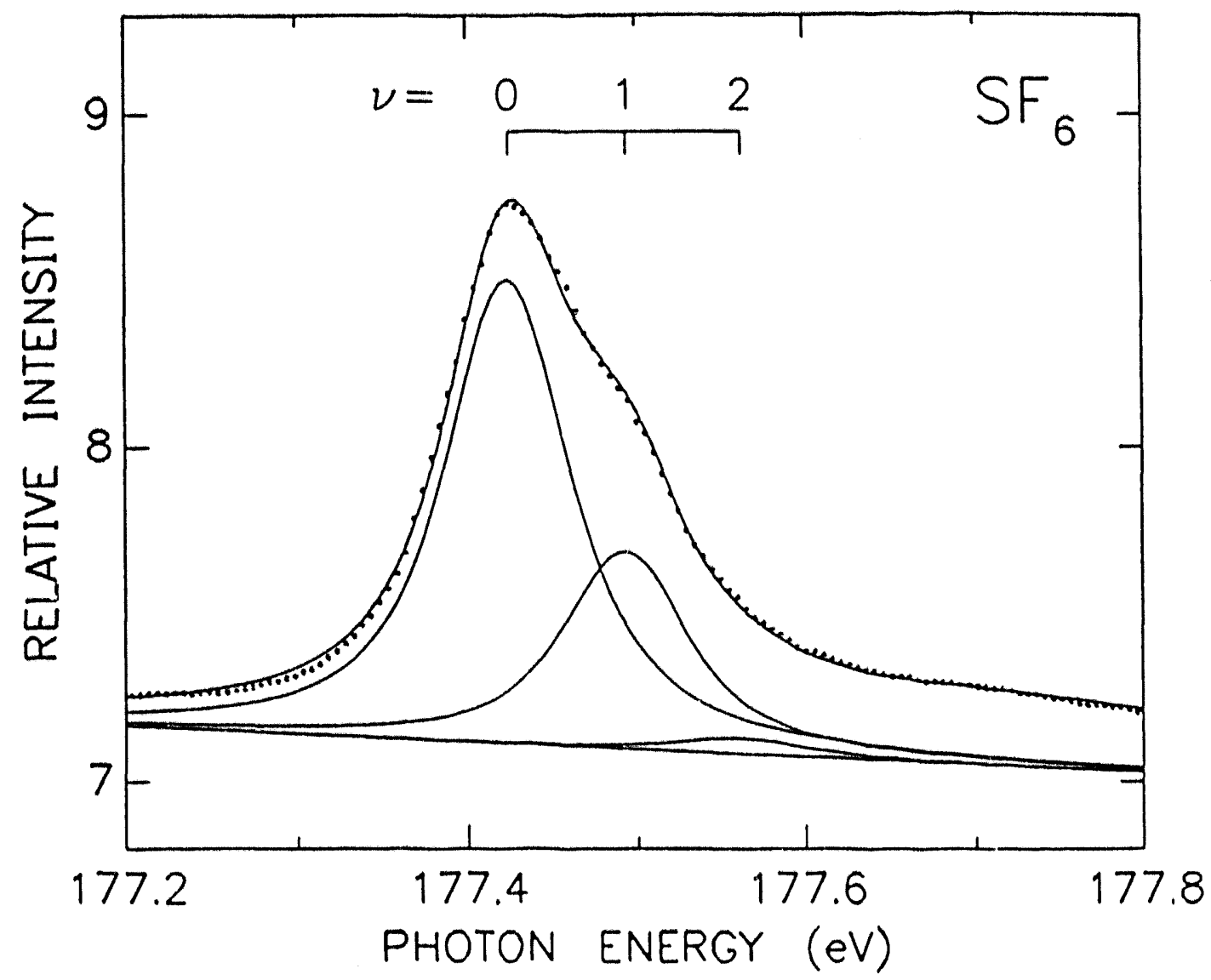

Figure V-7 


\section{CHAPTER VI: HIGH-RESOLUTION PHOTOIONIZATION SPECTRA NEAR THE SULFUR $\mathrm{L}_{1,2,3}$ THRESHOLDS: $\mathrm{H}_{2} \mathrm{~S}$ AND $\mathbf{D}_{\mathbf{2}} \mathrm{S}$}

\section{VI.A. Introduction}

The core levels of an atom exhibit a small but, in many cases, measurable sensitivity to chemical environment [1]. Besides its significance to a comprehensive understanding of molecular and condensed-phase electronic structure, this dependence is important in several widely-used spectroscopic techniques. Inner-shell spectroscopies such as Auger Electron Spectroscopy (AES), X-ray Emission Spectroscopy (XES), X-ray Photoelectron Spectroscopy (XPS), Electron-Energy Loss Spectroscopy (EELS) and Xray Absorption Near Edge Structure (XANES) are sensitive, each in its own way, to chemically-induced shifts in core-orbital energies and spatial distributions. As these techniques improve, there is an increasing ability to detect very subtle changes in core orbitals. With these developments comes the need to understand effects which were previously difficult or impossible to measure. In this chapter, analysis of high-resolution molecular photoionization spectra allows the measurement of the molecular-field splitting of a core level. This result demonstrates the potential of high-resolution core-level spectroscopies for the study of atomic-core structure in different chemical surroundings.

For this work, the high-resolution photoionization spectra of hydrogen sulfide, $\mathrm{H}_{2} \mathrm{~S}$, and its fully deuterated analog, $\mathrm{D}_{2} \mathrm{~S}$, have been measured near the sulfur $L_{1,2,3}$ ionization thresholds. A detailed understanding of the spectra is obtained by applying new approaches in data analysis. Previous photoabsorption measurements near the $L_{2,3}$ thresholds of $\mathrm{H}_{2} \mathrm{~S}$, as well as other third-row hydrides, yielded complicated spectra with many overlapping sharp lines preceded by a few broader features $[2,3]$. The latter features were assigned to one-electron transitions of a sulfur $2 \mathrm{p}$ electron into the two lowest 
unoccupied molecular orbitals [4,5]. Following the terminology used at lower photon [6] energies, these core-excited states are referred to here as "valence-shell states" (see Section I.D.2). The sharper peaks at higher energies were assigned to similar transitions into several series of atomic-like Rydberg orbitals leading up to the ionization thresholds. These Rydberg states, in particular, were difficult to assign fully due to limited resolution and statistics. In the present work, these limitations were overcome, and the assignment is now limited more by the complexity of the spectrum than by the quality of the data. The analysis presented here unambiguously assigns the higher Rydberg states converging on the sulfur $\mathrm{L}_{2,3}$ edges. A more tentative assignment of the lower Rydberg states is also given. Most important, however, is the determination of the core hole corresponding to each feature, and thus the term value of the associated Rydberg orbital. To accomplish this, a least-squares analysis was employed, which allowed the deconvolution of core-level and excited-orbital splittings.

The sulfur $2 p$ core orbitals are split into $2 p_{3 / 2}$ and $2 p_{1 / 2}$ levels by spin-orbit interaction. This $\approx 1.2 \mathrm{eV}$ splitting has been measured previously in $\mathrm{H}_{2} \mathrm{~S}$ by XANES, AES, and XPS $[3,7,8,9]$. The atomic $2 p_{3 / 2}$ level is doubly degenerate, but in the $C_{2 v}$ symmetry of the $\mathrm{H}_{2} \mathrm{~S}$ molecule, this degeneracy may be removed by the anisotropic molecular field. A recent comparison of high-resolution XPS and AES suggested that this splitting is $\approx 100 \mathrm{meV}$ in core-ionized $\mathrm{H}_{2} \mathrm{~S}$ [7]. In this report, the least-squares analysis confirms the molecular-field splitting of the sulfur $2 p_{3 / 2}$ level, and determines it to be 115 meV for the higher Rydberg states. Because the total observed linewidths of the Rydberg peaks are $\approx 70 \mathrm{meV}$ in this experiment, this splitting has a notable effect upon the spectrum.

Photoabsorption [10] and especially photoelectron spectroscopy [11] have been used to measure molecular-field splittings in the $3 \mathrm{~d}$ and $4 \mathrm{~d}$ levels of a number of elements. The molecular-field splitting of the $2 \mathrm{p}_{3} / 2$ core level has been predicted for the XANES spectra of $\mathrm{Cl}$ and $\mathrm{P}$ compounds, and has possibly been resolved for $\mathrm{PH}_{3}[12,13]$. The pronounced multiplet splitting of the $\left(\mathrm{S} 2 \mathrm{p}_{1 / 2,3 / 2}\right)^{-1} \mathrm{~b}_{1}{ }^{*}$ XANES resonances in $\mathrm{SO}_{2}$ 
indicates a sizable anisotropic influence on the sulfur $2 p$ levels in this electronic state, but no such effects are evident for the higher-energy (S 2p)-1 Rydberg resonances in $\mathrm{SO}_{2}$ [14]. The present work clearly resolves the 2p3/2 core level splitting in the XANES spectrum of $\mathrm{H}_{2} \mathrm{~S}$. With an lonization energy of $=170 \mathrm{eV}$, this is the deepest core-level for which this splitting has been measured.

Photoionization spectra of $D_{2} S$ were measured for the first time to aid in the assignment of the spectra. Transitions which include the excitation of vibrational modes are identified by a shift in energy upon deuteration of the molecule, because of the isotopic dependence of vibrational frequencies. Isotopic differences appear mainly in the region of the spectra between the valence-shell and upper Rydberg excitations. Spectral features in this region are characterized by narrow linewidths, like the Rydberg states al higher energies, and by extensive vibrational progressions, as observed for the valence-shell states at lower energies. For these reasons, and others, these features are assigned to transitions to "mixed orbitals", intermediate in spatial distribution and in energy between the valence and Rydberg orbitals (see Section I.D.3 and Ref. [6]).

Creation of a $2 p$ core hole may affect bonding within the molecule, but that influence should be nearly the same for any of the three non-degenerate $2 p$ core holes (i .e. the single $2 p_{1 / 2}$ hole and the two molecular-field-split $2 p_{3 / 2}$ holes). Therefore, the three non-degenerate excited states resulting from promotion of a $2 p$ electron into a particular final-state orbital should have essentially the same vibrational structure. The associated spectrum will contain a partially overlapping set of three vibrational progressions, of fset in energy from eachother, but otherwise identical. In principle, deconvoluting the contribution of the core level splitting to the spectrum will reveal the vibrational spacings corresponding to the excitation of each individual final-state orbital. In fact, because of overlapping vibrational progressions the resulting information is still too complicated for a definitive assignment and/or a Franck-Condon analysis.

Fine structure with an isotopic dependence is also observed in the broad, lower- 
energy features of the spectra. This regularly-spaced structure is only barely resolved, due to the large natural linewidths of the valence-shell excitations. Vibrational excitation is expected for these transitions into unoccupied molecular orbitals, since at least one of the orbitals accessed is strongly antibonding $[4,15]$. Putting an electron into an antibonding orital tends to lengthen bonds and thus induce vibrational excitations, unless there are strong compensating effects from the creation of the core hole. Recent studies showed that rapid dissociation is an important decay process for these core-excited valence-shell states $[16,17,18]$. Interpretation of the vibrational structure is complicated hy this dissociation and by the possibility of vibronic interaction hetween the several electronic states in this energy range.

Photolonization spectra of $\mathrm{H}_{2} S$ and $\mathrm{D}_{2} \mathrm{~S}$ in the region of the sulfur $\mathrm{L}_{1}$ threshold are presented here for the first time. These spectra are very similar to the corresponding spectra of $\mathrm{H}_{2} \mathrm{~S}$ in the sulfur is region [19]. Due to the short lifetime of the $2 \mathrm{~s}$ core hole. the natural linewidths are large in these spectra and fine structure is not observed.

\section{VI.B. Experimental}

The SX-700/II monochromator, with the 2442 line/mm grating used in the first order of diffraction, produced the optimum combination of flux and energy resolution. For the measurements near the sulfur $L_{2,3}$ edges, the resolution was $\approx 30 \mathrm{meV}$ (Gaussian Full Width at Half Maximum (FWHM]). Al the sulfur $L_{l}$ edge, the resolution was $=45$ meV. The gas cell contained $\mathrm{H}_{2} \mathrm{~S}$ (Messer Griesheim. $99.3 \%$ ) or $\mathrm{D}_{2} \mathrm{~S}$ (CIL. 98\%) at a typical pressure of $0.10 \mathrm{mbar}$. A $1000 \AA$ carton window separated the gas cell from the ultra-high vacuum of the monochromator. 


\section{vi.c. Overview of results}

Fig. VI-I shows an overview of the $\mathrm{H}_{2} \mathrm{~S}$ photoionization spectrum in the 160-240 $\mathrm{eV}$ photon energy range. Intense resonances are apparent below the sulfur $L_{1}$ ionization threshold at $=235 \mathrm{eV}$ and the sulfur $L_{2,3}$ thresholds at $=171 \mathrm{eV}$. The region below the $L_{2,3}$ thresholds contains an extensive series of peaks, shown in greater detail in Figs. 2 and 3. The inset of Fig. VI-I shows a higher-resolution spectrum of the weak features in the range 178.56-185.56 eV. Note that the energy scale of the inset is five times that of the main spectrum. Similar structure is also observed in the corresponding spectrum of $\mathrm{D}_{2} \mathrm{~S}$. These peaks are attributed to multi-electron excitations in which a valence electron and a sulfur $2 p$ core electron are simultaneously excited to valence and/or Rydberg orbitals. Analogous features are observed in the core-level absorption spectra of many atoms and molecules 120.211 .

Fig. VI-2 shows the high-resolution spectra of $\mathrm{H}_{2} \mathrm{~S}$ and $\mathrm{D}_{2} \mathrm{~S}$ in the range $=167-172$ eV. For both spectra, the complicated pattern of features is assigned to one-electron excitations from the sulfur $2 p$ core levels to several series of intermediate and Rydbers orbitals. These ortitals may be split into several components by the anisotropic molecular field. Additionally, each final stare orbital is accessed by transitions from the three nondegenerate sulfur $2 p$ core levels. The large number of dipole-allowed transitions is further increased by the possibility of vibrational excitations accompanying the electronic excitation. Differences between the two spectra are most prominent in Fig. VI-2 for the lower energy range $-167-168 \mathrm{cV}$, i.e. the region of transitions to mixed states. These differences are expected in the vibrational sidebands of electronic transitions due to the strong isotopic dependence of vibrational frequencies. The energy scales of the $\mathrm{H}_{2} \mathrm{~S}$ and $\mathrm{D}_{2} \mathrm{~S}$ spectra were aligned by assuming the $\left(2 \mathrm{p}_{1 / 2}\right)^{-1}$ sd Rydherg state (at $170.94 \mathrm{eV}$ in Fig. VI-2) has the same energy in both molecules.

The lowest-energy features of Fig. VI-1 are shown with much higher energy 
resolution in Fig. VI-3, along with the corresponding spectrum of $\mathrm{D}_{2} \mathrm{~S}$. These peaks are assigned to one-electron transitions from the sulfur $2 p$ core level in the unoccupied $6 a_{1}$ and $3 b_{2}$ molecular orbltals. Both of these transitions are split by the non-degeneracy of the sulfur $2 p$ core electrons. The weak fine structure apparent in both spectra is also shown in Fig. VI-3 with an expanded intensity scale. These regularly spaced features, seen here for the first time, are attributed to the excitation of vibrations in the final state. Small isotopic shifts in the overall peak wideh and position were also observed, as indicated by the vertical lines.

The photolonization spectrum of $\mathrm{H}_{2} \mathrm{~S}$ near the sulfur $L_{1}$ edge is shown in Fig. VI.

4. The series of peaks arises from transitions in which a sulfur 28 electron is promoted to a valence or Rydherg orbital; these are analogous to the sulfur $2 \mathrm{p}$ excitations in the spectra of Figs. 2 and 3. However the lifetime of the sulfur $2 s$ cone hole is much shorter than that of the sulfur $2 p$ core hole because of the rapid Coster-Kronig decay process [22]. This difference is reflected in the larger overall linewidths of the resonances near the $L_{1}$ edge.

\section{VI.D. Sulfur L2,3 Edges: Non-degeneracy of the sulfur $2 p$ core level}

To understand the molecular-field effects upon the sulfur $2 p$ core levels, it is first necessary to review the hehavior of these core levels in the atomic case. Consider, then, an initially closed-shell atom, e.g. Ar, in which a 2 p electron is excited to a Rydherg orbital $\mathrm{n}$, where $\mathrm{n}$ and $\mathrm{f}$ are the principal and angular-momentum quantum numbers of the orbital. Following the discussion in Ref. [23], the Hartree-Fock energy of this N-electron system is given by

$$
\mathrm{E}=\langle\Psi|H| \Psi\rangle=-\sum_{i}^{N}\left\langle\chi_{i}\left|\frac{\nabla_{i}^{2}}{2}+\frac{Z_{1}}{r_{i}}\right| \chi_{i}\right\rangle+\sum_{j<i} \sum_{i}^{N}\left(J_{i j}-K_{i j}\right)+\sum_{i}^{N}\left\langle\chi_{i}\left|\xi_{i}\left(r_{i}\right) \varepsilon_{i} \cdot s_{i}\right| \chi_{i}\right\rangle
$$


where the total wavefunction $\Psi$ is the antisymmetrized product of $N$ single-electron spin orbitals, $\chi_{i}$. In Eq. (VI-1), $Z$ is the nuclear charge, $r_{i}$ is the position operator for electron $i, J_{i j}$ is the direct (Coulomb) integral hetween the $i$ th and jth electrons. $K_{i j}$ is the exchange integral between the $i$ th and $j$ th electrons, and $\xi_{i}\left(r_{i}\right) \ell_{i} s_{i}$ is the spin-orbit interaction of the ith electron. The terms which are important here for the final-state splittings are the spinortit interaction energies of the $2 p$ electrons, and the two-electron exchange terms, $K_{i j}$ in which one electron is the excited electron and the other is one of the remaining $2 p$ electrons. In the limit of ne gligible spin-orbit interaction. LS (Russell-Saunders) coupling applies. In the limit of negligihle exchange interaction. $j-j$ coupling applies. In the general case, an intermediate coupling must be introduced which lies between these two extremes [24]. For pure $f-j$ coupling, the two possible excited states are labeled $\left(2 p_{3} / 2\right) \cdot 1 n\left(\right.$ and $\left(2 p_{1 / 2}\right)^{-1} n h$. Their energy difference is given by comparing the last term of Eq. (VI-1) for the two different core configurations. The statistical intensity ratio of transitions to the two states is $2: 1\left(2 p_{3} / 2^{-1}: 2 p_{1 / 2} \cdot 1\right)$.

The spin-orbit interaction of the $2 p$ electrons is not influenced by the excited electron, and thus is independent of $\mathrm{n}$ and $\mathrm{Q}$. The exchange interaction, however, is strongly dependent on the spatial overlap of the $2 p$ and $n c$ orbitals. For large enough values of $n+l$, exchange effects are negligible and $j \cdot j$ coupling is valid. For the lower values of $n+c$, the exchange interaction is somewhat larger, and intermediate coupling may apply. Several examples show that the effect of the exchange interaction in this situation is mainly to modify the intensity ratio of the two transitions, without changing the observed energy difference very much [25]. The transition to the $\left(2 \mathrm{p}_{3} / 2\right)^{-1} \mathrm{n}$ s state loses intensity, reflecting its correlation with the dipole-fortidden triplet state in the limit of LS coupling.

This discussion is readily extended to closed-shell molecules. One difference is the stronger spatial overlap which occurs, in a molecule, hetween a valence-shell-excited electron and a core hice, as compared to the limited overlap of electrons in atomic orbitals with differing $n$. Because of this. molecular core-excited valence-shell states will generally 
show larger exchange effects than are seen in the corresponding atomic states. Another complication in the molecular case is that the spherical symmetry of the free atom is removed. Specifically, the $\mathrm{H}_{2} \mathrm{~S}$ molecule has $\mathrm{C}_{2 v}$ symmetry, which splits the sulfur $2 p$ core level into three energy levels. In the atomic case, the spin-orbit interaction splits the $2 p$ core level into the $2 p_{1 / 2}$ and the twofold degenerate $2 p_{3 / 2}$ levels. When the influcnce of the anisotropic molecular field of $\mathrm{H}_{2} \mathrm{~S}$ is also considered, the degeneracy of the sulfur $2 \mathrm{p}_{3 / 2}$ level is removed. The two resulting levels may be designated $4 e_{1 / 2}$ and $5 e_{1 / 2}$ in the extended point group which applies to $\mathrm{C}_{2 v}$ symmetry, In this chapter these sulfur core levels are labeled $2 p_{3 / 2}-4 e_{1 / 2}$ and $2 p_{3 / 2}-5 e_{1 / 2}$, and the corresponding ionization thresholds are $L_{3}-4 e_{1 / 2}$ and $L_{3}-5 e_{12}$, where the latter core hole is the shallower of the two and thus the latter threshold has the lower energy of the two. The abbreviated label $2 p_{3} / 2$ refers to hoth levels. The deepest sulfur $2 p$ level is still best described as $2 p_{1 / 2}$ hecause the molecular-field splitting is much smaller than the spin-orbit splitting. For convenience, the difference between the energy of a $\left(2 p_{1} / 2\right)^{-1}$ stute and the average energy of the corresponding $\left(2 p_{3 / 2}\right)^{-1}$ states will the called the mean spin-orbit splitting. although this quantity may in fact be influenced by the molecular field and by the (direct and exchange) Coulombic interactions of the excited electron with the core hole. Note that the molecular field refers to the anisotropic electrostatic field felt by the sulfur $2 p$ electrons, which includes a contribution from the direct interaction with the excited electron.

It is important to recognize the distinction hetween the energy splittings of the sulfur $2 p$ core levels in the core-excited ion, as measured by XPS, and the splittings which are observed in photoabsorption (or photoionization). In XPS, in the high kinetic-energy limit, the splittings ohserved are those of the core levels in the electronically-relaxed coreexcited ion. In near-edge photoabsorption measurements, however, one must also account for the influence of the excited electron. As in the atomic case, the exchange interaction, $K_{i j}$, between the excited electron and the core electrons may modify the observed splittings. However there are three configurations of the molecular core electrons, corresponding to 
the three possible core holes, rather than two. Thus there are two splittings which may be modified, rather than one. In $\mathrm{H}_{2} \mathrm{~S}$ it is also possible for the direct interaction, $\mathrm{F}_{i j}$, between the excited electron and the $2 p$ electrons to influence the observed splittings. This is possible because, in contrast to the atomic case, the $2 p$ electrons occupy non-identical spatial orbitals and thus the direct Coulombic repulsion "between the excited electron and the three possible configurations of core electrons may vary. Both the exchange and direct interactions will be largest for states in which the excited electron has strong spatial overlap with the core. Dipole-allowed transitions to such states should be intense and, in the absence of strong shape resonances, should have the largest term values (smallest excitation energies).

\section{VI.E. Sulfur L2,3 edges: Rydberg and mixed excitations}

\section{General considerations}

The geometric [26] and electronic structures of ground-state $\mathrm{H}_{2} \mathrm{~S}$ and $\mathrm{D}_{2} \mathrm{~S}$ are very similar. Therefore, differences between the photoexcitation spectra of the two molecules are expected primarily as a result of the large differences in vibrational frequencies between the two molecules in their final excited states. Fig. VI-2 shows high-resolution spectra of $\mathrm{H}_{2} \mathrm{~S}$ and $\mathrm{D}_{2} \mathrm{~S}$ in the region of the intermediate and Rydberg excitations. Upon comparison of the spectra, it is apparent that isotopic differences lie mainly in the lowest third of the energy range shown. Therefore, some electronic transitions in this lower range must include vibrational excitations. Vibrational sidebands are often observed for transitions to core-excited molecular Rydberg states $[20,21,27,28]$. However, the large isotopic differences seen here indicate more extensive vibrational excitations than are normally seen for Rydberg states. The excited electron may therefore be better described as possessing both Rydberg-orbital and molecular-orbital character. These states are referted to here as 
"mixed" or intermediate states, as mentioned above.

Further comparison shows that the two spectra are nearly identical in the upper third of the energy range. An immediate conclusion is that above $\approx 169 \mathrm{eV}$ the spectra contain, at most, minor contributions from vibrational sidebands. Thus, the rich fine structure of the upper region arises only from electronic excitations, without vibrational excitation. By comparison, the corresponding region of the $\mathrm{L}_{2,3}$ absorption spectrum for the isoelectronic rare gas atom, argon, $[29,30]$ is much less complex. One complicating factor for $\mathrm{H}_{2} \mathrm{~S}$ is the molecular-field splitting of the sulfur $2 \mathrm{p}_{3 / 2}$ core level. Another consideration is the influence of the $\mathrm{C}_{2 \mathrm{v}}$-symmetry molecular field upon the Rydberg orbitals and upon the dipole-selection rules.

The group-theoretical treatment of electronic transitions in $\mathrm{H}_{2} \mathrm{~S}$ summarized here is based upon the thorough discussion in Ref. [31]. When atomic orbitals are resolved into $\mathrm{C}_{2 v}$ molecular orbitals, $s$ becomes $a_{1}, p$ becomes $a_{1}+b_{1}+b_{2}$, and $d$ becomes $2 a_{1}+a_{2}+b_{1}+b_{2}$. Note the complete removal of spatial degeneracy for the $\mathrm{p}$ and $d$ orbitals. For the randomly-oriented closed-shell molecules of this experiment, the dipole-selection rules allow transitions to $A_{1}, B_{1}$, or $B_{2}$ molecular final states. The characters of the molecular states which correspond to a given atomic configuration are obtained by multiplying the spin and space characters of all the open-shell electrons. For example, the $2 p^{-1} n$ atomic $c^{\prime n f i g u r a t i o n ~ c o r r e s p o n d s ~ t o ~ a ~ s p a c e ~ c h a r a c t e r ~}\left(a_{1}+b_{1}+b_{2}\right) \times a_{1}=a_{1}+b_{1}+b_{2}$, and a total spin of $S=1 / 2 \pm 1 / 2=0,1$. In $C_{2 v}$ symmetry, $S=0$ becomes $a_{1}$ and $S=1$ becomes $a_{2}+b_{1}+b_{2}$. Thus the total molecular states are $\left(a_{1}+a_{2}+b_{1}+b_{2}\right) \times\left(a_{1}+b_{1}+b_{2}\right)=$ $3\left(A_{1}+A_{2}+B_{1}+B_{2}\right)$. Therefore ground-state transitions are dipole-allowed to 9 final states, $3\left(\mathrm{~A}_{1}+\mathrm{B}_{1}+\mathrm{B}_{2}\right)$, corresponding to the $(2 \mathrm{p})^{-1} \mathrm{~ns}$ atomic configuration for a single value of $\mathrm{n}$. It can be shown that one subset of dipole-allowed states, $A_{1}+B_{1}+B_{2}$, corresponds to each of the three possible $2 p$ core holes: $2 p_{3 / 2}-5 e_{1 / 2}, 2 p_{3 / 2}-4 e_{1 / 2}$, and $2 p_{1 / 2}$. This example does not show that the ns atomic orbital somehow splits into three components in $\mathrm{H}_{2} \mathrm{~S}$; the tripling results from the relaxation of atomic angular-momentum constraints in the 
molecular dipole selection rules. Similar analyses show that the $(2 p)^{-1} \mathrm{np}$ and $(2 \mathrm{p})^{-1}$ nd configurations correspond to $9\left(A_{1}+A_{2}+B_{1}+B_{2}\right)$ and $15\left(A_{1}+A_{2}+B_{1}+B_{2}\right)$, respectively. Hence, three and five subsets, respectively, of dipole-allowed states, $A_{1}+B_{1}+B_{2}$ correspond to each of the three possible $2 p$ core holes. For np and nd, the removal of the spatial degeneracy of the Kydberg orbital and the possibility of spin-orbit splitting of the Rydberg orbital also contribute to the large number of dipole-allowed final states.

By comparison, for the corresponding excitations in $\mathrm{Ar}$, there are dipole-allowed transitions only to $2 p^{-1} n s$ and $2 p^{-1}$ nd configurations. These result in two and three triplydegenerate states, respectively, totaling 15 states altogether, for a single value of $n$. In $\mathrm{H}_{2} \mathrm{~S}$, for the same shell $\mathrm{n}$, there are dipole-allowed transitions to 81 singly-degenerate states! In light of these numbers, it becomes evident that the Rydberg spectra observed for $\mathrm{H}_{2} \mathrm{~S}$ and $\mathrm{D}_{2} \mathrm{~S}$ are in fact much simpler than they would be if all possibls states were resolved. This is not surprising, because the Rydberg orbitals, which extend far away from the molecule, mostly feel only a weak molecular field. The higher Rydberg orbitals, in particular, behave much like atomic orbitals and the corresponding transitions are readily assigned. And even purely atomic splittings might not be resolved. For example, in the Ar $\mathrm{L}_{2,3}$ spectrum, the spin-orbit splitting of the nd Rydberg orbitals is too small to be observed [30].

The interpretation of the spectra in Fig. VI-2 is facilitated by a detailed analysis of the data. A least-squares fitting routine was employed to extract peak positions, intensities, etc. from the data, within the constraints of the assumed functional form of the spectra. The same approach was used for both the $\mathrm{H}_{2} \mathrm{~S}$ and $\mathrm{D}_{2} \mathrm{~S}$ spectra. Voigt functions modeled the lineshapes of the peaks, with fixed Gaussian contributions to represent the estimated resolution and adjustable Lorentzian contributions to represent lifetime broadening. The edge jumps were modeled by arctangent functions, broadened by convolution with the Gaussian resolution function. To account for the "pile-up" of unresolved transitions just below the ionization threshold, [32] the $\mathrm{L}_{2}$-edge arctangent function was shifted $-172 \mathrm{meV}$ 
relative to the $\mathrm{L}_{2}$ threshold energy determined from the Rydberg analysis. In addition, a broad Voigt function centered on the arctangent function was necessary for a good fit to the lineshape. The resulting model of the $\mathrm{L}_{2}$ edge jump was shifted to the lower energies corresponding to the $\mathrm{L}_{3}-4 \mathrm{e}_{1 / 2}$ and $\mathrm{L}_{3}-5 \mathrm{e}_{1 / 2}$ edge jumps, using the splittings determined from the upper range of Rydberg transitions. The fit also included a linear background and, near the lower-energy limit, a contribution from the high-energy tail of the broad valence-shell transitions of Fig. VI-3. The results of this least-squares analysis are presented in Tables I and II and are plotted with the data in Fig. VI-2. For each spectrum in Fig. VI-2, one subspectrum shows the edge jumps and the tail of the valence-shell transition. The other three subspectra show the transitions corresponding to the three possible $2 p$ core-level vacancies. Further details of the data analysis are given below, as necessary.

\section{Upper range: Rydberg states}

The region of the Rydberg spectrum above $170.4 \mathrm{eV}$ is the simplest to interpret because it lies above the $\mathrm{L}_{3}-4 \mathrm{e}_{1 / 2}$ and $\mathrm{L}_{3}-5 \mathrm{e}_{1 / 2}$ ionization thresholds. All the peaks observed must therefore correspond to excitations from the sulfur $2 \mathrm{p}_{1 / 2}$ core-level. This conclusion results immediately from an estimate of the spin-orbit splitting of the sulfur $2 \mathrm{p}$ core level; it does not depend on any other details of the analysis. Above $170.8 \mathrm{eV}$, the interpretation is further facilitated by assuming that atomic designations apply, so that s-,

p-, and d-symmetry Rydberg series may be identified. Note that the reduced symmetry of $\mathrm{H}_{2} \mathrm{~S}$ relaxes the atomic dipole selection rule which forbids $2 \mathrm{p}$ to np transitions. For the region above $170.8 \mathrm{eV}$, the least-squares analysis of the $\mathrm{H}_{2} \mathrm{~S}$ and $\mathrm{D}_{2} \mathrm{~S}$ spectra assumed the Rydberg formula. Excellent fits were obtained using the quantum defects $\delta_{\mathrm{p}}=1.63$ and $\delta_{\mathrm{d}}$ $=0.32$, and $\mathrm{E}_{\mathrm{IP}}=171.564 \mathrm{eV}$ for the sulfur $\mathrm{L}_{2}$ ionization threshold. The assignment of these quantum defects to the p- and d-Rydberg series is discussed below, in Sec. VI.E.4.b, 
along with a comparison of the derived quantum defects to corresponding values for some isoelectronic systems.

The region between $170.3 \mathrm{eV}$ and $170.8 \mathrm{eV}$ is somewhat more complicated. Two prominent peaks are observed at $170.315 \mathrm{eV}$ and $170.534 \mathrm{eV}$, near the energies predicted by the Rydberg formula for the $\left(2 \mathrm{p}_{1 / 2}\right)^{-1} 5 \mathrm{p}$ and $\left(2 \mathrm{p}_{1 / 2}\right)^{-1} 4 \mathrm{~d}$ states, but both show highenergy shoulders (See Fig. VI-2 and Table VI-1). The spectra of $\mathrm{H}_{2} \mathrm{~S}$ and $\mathrm{D}_{2} \mathrm{~S}$ are very similar in this region, indicating that vibrational sidebands are not present, beyond the exception described below. The energies discussed here are for $\mathrm{H}_{2} \mathrm{~S}$, but the corresponding values for $\mathrm{D}_{2} \mathrm{~S}$ are nearly the same (see Table VI-1). The most prominent peak associated with the $\left(2 \mathrm{p}_{1 / 2}\right)^{-1} 4 \mathrm{~d}$ state has a transition energy $26 \mathrm{meV}$ smaller than predicted by $\delta_{\mathrm{d}}$. The two shoulders lie $70 \mathrm{meV}$ and $136 \mathrm{meV}$ above the prominent peak. The second shoulder is teniatively assigned to a transition to the $6 \mathrm{~s}$ Rydberg orbital, with a resulting quantum defect of $\delta_{\mathrm{G}}=2.10$. The first shoulder is assigned to a transition associated with the $4 \mathrm{~d}$ orbital. As discussed above, the influence of the molecular field can remove the spatial degeneracy of the d-orbital and relax angular-momentum restrictions on transitions. Many transitions may be observed which are derived from a single atomic transition, and the assignments given in Table VI-1 stress that correspondence wherever possible. The prominent peak and large shoulder associated with the ( $\left.2 \mathrm{p}_{1 / 2}\right)^{-1} 5 \mathrm{p}$ state have transition energies $49 \mathrm{meV}$ and $11 \mathrm{meV}$ smaller than predicted by $\delta_{\mathrm{p}}$. Note that these peaks lie at low enough energies to include contributions from states with a $2 p_{3 / 2}-4 e_{1 / 2}$ core hole, but no fine structure is expected in the region immediately below an ionization threshold.

The detailed fits of the $\left(2 p_{1 / 2}\right)^{-1} 5 p, 4 d$ region showed a notable difference between the $\mathrm{H}_{2} \mathrm{~S}$ and $\mathrm{D}_{2} \mathrm{~S}$ spectra which is not easily detected by eye; the low-intensity feature at $170.466 \mathrm{eV}$ in $\mathrm{H}_{2} \mathrm{~S}$ is shifted $-43 \mathrm{meV}$ in $\mathrm{D}_{2} \mathrm{~S}$ (see Table VI-1). This shoulder is assigned to a vibrational sideband of the intense $5 \mathrm{p}$-derived transition at $170.315 \mathrm{eV}(170.309 \mathrm{eV})$ in $\mathrm{H}_{2} \mathrm{~S}\left(\mathrm{D}_{2} \mathrm{~S}\right)$. The derived vibrational spacing for the excited state is $153 \mathrm{meV}$ (114 meV) for 
$\mathrm{H}_{2} \mathrm{~S}\left(\mathrm{D}_{2} \mathrm{~S}\right)$. These values suggest an assignment to the $v_{2}$ bending mode, with a groundstate spacing of $147 \mathrm{meV}(106 \mathrm{meV})$ [33]. Other vibrational results are discussed below in Sec IV D. Another low-intensity feature in this region, which lies at $170.807 \mathrm{eV}$ in $\mathrm{H}_{2} \mathrm{~S}$, is shifted $-16 \mathrm{meV}$ in $\mathrm{D}_{2} \mathrm{~S}$. Neither the isotopic shift nor the spacing of the nearby states suggests a simple vibrational assignment for this feature. It could be attributed to a molecular-field-split component of the nearby $\left(2 \mathrm{p}_{1 / 2}\right)^{-1} 6 \mathrm{p}$ state, but that implies a larger splitting than observed for the $\left(2 p_{1 / 2}\right)^{-1} 5 p$ state and does not explain the isotopic energy dependence.

\section{Lower range: Rydberg and mixed states}

\section{a. Approach used for analysis}

In the range 166.8-170.3 eV, interpretation of the $\mathrm{H}_{2} \mathrm{~S}$ and $\mathrm{D}_{2} \mathrm{~S}$ spectra is more difficult. An independent least-squares fit of the $\approx 65$ transitions observed in this range for the two spectra would require an adjustable energy, intensity, and linewidth for each transition. Such an analysis could produce near-perfect fits of the two spectra in this region but would not provide much understanding of the nature of the transitions. An alternative approach was employed instead. The set of adjustable parart ,ers was reduced by making the small set of assumptions described below, which were carefully selected to represent the essential physics of the problem. A least-squares analysis based on these restrictions gave good unique fits of the two spectra when the molecular-field splitting of the sulfur $2 p_{3 / 2}$ core levels was included. Much poorer fits were obtained if the $2 p_{3 / 2}$ levels were assumed to be degenerate. Beyond this important result, the analysis correlated each individual feature with one of the three possible $2 \mathrm{p}$ core holes, thus deconvoluting the Rydberg-orbital structure from the core-level structure. Due to the complexity of the spectrum, the large number of adjustable parameters, and the limited accuracy of the fit, not 
all the results of this deconvolution are reliable, as discussed in detail below. However, a general understanding of the spectrum is obtained, which might not be apparent otherwise.

For the least-squares analysis of the Rydberg spectra, the following assumptions were applied. (1) The energy spacing of transitions from the three $2 \mathrm{p}$ core levels to a single Rydberg orbital, i.e. the observed splitting of the three core levels, is independent of which Rydberg orbital is accessed (but see below for a major exception). (2) The spacing of these triplets is equal for $\mathrm{H}_{2} \mathrm{~S}$ and $\mathrm{D}_{2} \mathrm{~S}$. (3) For every pair of transitions $(A, B)$ from the two $2 p_{3 / 2}$ core levels to one Rydberg orbital, the two peaks $A, B$ have equal intensities. Thus, for the triplet of transitions (A,B,C) to a single Rydberg orbital, A and B have equal intensity, but $C$, the transition from the $2 \mathrm{p}_{1 / 2}$ level, can have a different intensity. (4) The ratio, $X$, of the intensities of peaks $A$ and $C$ (i.e. $X \equiv$ [intensity of $A$ ] / [intensity of $C$ ]) may vary for different Rydberg orbitals, but must be the same for corresponding triplets in the $\mathrm{H}_{2} \mathrm{~S}$ and $\mathrm{D}_{2} \mathrm{~S}$ spectra. (Note that if the intensity ratio between transitions from the $2 p_{1 / 2}$ level and the $2 p_{3 / 2}$ levels follows the $j-j$ coupling "statistical" prediction of $1: 2$, the ratio $X$ will equal unity.) (5) Each triplet of peaks $(A, B, C)$ must have the same linewidth, which does not have to be the same for corresponding triplets in the $\mathrm{H}_{2} \mathrm{~S}$ and $\mathrm{D}_{2} \mathrm{~S}$ spectra.

Assumption (1) is only valid if the exchange interaction between the excited electron and the core hole is small, as mentioned in Sec. VI.D. This should apply for the higher Rydberg states, but may be less accurate for the lower Rydberg states. Indeed, this assumption had to be relaxed to allow a reasonable fit of the lowest-energy features of Fig. VI-2, the mixed states, as discussed below. Assumption (2) is valid if the electronic and geometric structure of the final state is essentially the same in $\mathrm{H}_{2} \mathrm{~S}$ and $\mathrm{D}_{2} \mathrm{~S}$. Because the splitting of the $2 p_{3 / 2}$ level is small, the $2 p_{3 / 2}-4 e_{1 / 2}$ and $2 p_{3 / 2}-5 e_{1 / 2}$ core orbitals must have very similar spatial distributions, supporting assumption (3). Assumption (4) applies because corresponding Rydberg orbitals in $\mathrm{H}_{2} \mathrm{~S}$ and $\mathrm{D}_{2} \mathrm{~S}$ should have essentially the same spatial distributions. Note that a deviation of the intensity ratio $\mathrm{X}$ from unity indicates a departure from the statistical ratio, which can arise even from a relatively small exchange 
interaction between the core hole and excited electron. $\mathrm{X}$ is allowed to vary for different Rydberg orbitals, since differences in the spatial distributions of the various Rydberg orbitals can result in different interactions with the core hole. As discussed below, assumption (4) is not applied to the mixed states, because the correspondence of these features between $\mathrm{H}_{2} \mathrm{~S}$ and $\mathrm{D}_{2} \mathrm{~S}$ is difficult to determine. Assumption (5) is only valid if the three core holes decay at the same rate for a given Rydberg excitation. This assumption should apply for the two $2 p_{3 / 2}$ core holes, but is not expected to be entirely valid for $2 p_{1 / 2}$ vs. $2 p_{3 / 2}$ core holes, particularly for the lower Rydberg states. However, this restriction was necessary in keep the number of adjustable parameters in the analysis manageable, and the data showed no evidence of deviations from this assumption.

For the analysis, the $\mathrm{H}_{2} \mathrm{~S}$ and $\mathrm{D}_{2} \mathrm{~S}$ data sets were combined to best exploit assumptions (2) and (4). The fits of the upper Rydberg region, discussed above in Sec. VI.E.2, were included in the analysis. Note that this uppermost part of the spectrum, $170.3-171.4 \mathrm{eV}$, is essentially reproduced twice at lower energies in the fit, to represent the sequence of states approaching the $\mathrm{L}_{3}-4 \mathrm{e}_{1 / 2}$ and $\mathrm{L}_{3}-5 \mathrm{e}_{1 / 2}$ edges. This is seen in the subspectra of Fig. VI-2, where the dashed line shows features attributed to $2 \mathrm{p}_{1 / 2}$ core-hole states, the solid line shows features attributed to $2 \mathrm{p}_{3 / 2}-4 \mathrm{e}_{1 / 2}$ core-hole states, and the dotted line shows features attributed to $2 \mathrm{p}_{1 / 3}-5 \mathrm{e}_{1 / 2}$ core-hole states. Ideally, for each spectrum the three subspectra should be equivalent except for a shift in energy due to the splitting of the $2 p$ levels. To obtain a reasonable fit, however, the ratios $X$ for each feature in the subspectra were independently varied within the range $0.20-2.50$. The ratios obtained are shown in Tables I and II. For most of the intense features, the derived ratio was close to one. But for some features, including a few intense peaks, significant deviations of $\mathrm{X}$ from unity were necessary to obtain a good fit. This is reflected in the subspectra of Fig. VI-2, where the dashed line is, in some places, different from the solid or dotted line. Of course the latter two lines have identical shapes, as required by assumption (3). Some of these deviations of $X$ from unity may represent real ratios of transition intensities in the 
spectrum. Others might be a result of the limited validity of the assumptions (1)-(5), which could force unrealistic values onto some adjustable parameters. Because of this, and also because of the imperfect fit to the data, some of the quantitative results of this least-squares analysis may be unreliable. The results discussed below were selected with these restrictions in mind, and are believed to be valid.

\section{b. Results of analysis}

Results of the least-squares analysis based on this approach are given in Tables I and II and are plotted as the fits and subspectra in Fig. VI-2. Due to the large number of adjustable parameters, a simultaneous least-squares fit was not possible. Instead, alternating subsets of parameters were adjusted reiteratively until a good fit was obtained. As a result, uncertainties could not be calculated for the final fit parameters. Some uncertainties have been estimated, however. The energies, intensities, and intensity ratios (X, as defined above) from the fits are shown in Tables I and II. The only parameters not tabulated are the linewidths of the individual peaks, which have typical values from $75 \mathrm{meV}$ to $100 \mathrm{meV}$ (Voigt function FWHM). Linewidths are discussed in more detail below, in Sec. VI.G.

Tables I and II give energies of the $\left(2 \mathrm{p}_{1 / 2}\right)^{-1}$ core-excited states. The term values are obtained by subtraction from the derived sulfur $\mathrm{L}_{2}$ threshold energy of $171.564 \mathrm{eV}$. The energy of a particular $\left(2 p_{3 / 2}-4 e_{1 / 2}\right)^{-1}$ or $\left(2 p_{3 / 2}-5 e_{1 / 2}\right)^{-1}$ core-excited state can be obtained from Table VI-1 by subtracting the term value of the corresponding $\left(2 \mathrm{p}_{1 / 2}\right)^{-1}$ state from the derived values of the $L_{3}-4 e_{1 / 2}$ and $L_{3}-5 e_{1 / 2}$ threshold energies; $170.418 \mathrm{eV}$ and $170.303 \mathrm{eV}$, respectively. For the mixed states listed in Table VI-2 the term values are derived by assuming the same $L_{2}$ threshold energy as in Table VI-1. Because different core-level splittings apply for these states (see below), the effective $L_{3}-4 e_{1 / 2}$ and $L_{3}-5 e_{1 / 2}$ threshold energies are $170.471 \mathrm{eV}$ and $170.432 \mathrm{eV}$, respectively. 
The band of features in the range $167.0-168.2 \mathrm{eV}$, are of special interest because of their dissimilarity in the $\mathrm{H}_{2} \mathrm{~S}$ and $\mathrm{D}_{2} \mathrm{~S}$ spectra. Since no sharp, prominent features are observed at lower energies, l.e. in the range $165.8-167.0 \mathrm{eV}$ (see Fig. V1.3), the transitions within this band must access $\left(2 p_{3 / 2}\right)^{-1}$ core-excited states. The corresponding band of $\left(2 \mathrm{p}_{1 / 2}\right)^{-1}$ states should lie approximately in the range 168.2-169.4 eV, possibly overlapping some $\left(2 p_{3 / 2}\right)^{-1}$ core-excited Rydberg states. This rough assignment is supported by the observation of notable isotopic differences around 168.7-169.2 eV. The large isotopic differences of these two bands indicate that extensive vibrational excitations accompany the electronic excitations. Such strong vibrational excitations, in turn, indicatc that the orbital accessed by the transition has some valence character, rather than residing almost entirely outside the molecule. Thus the features belonging to these two bands are assigned to transitions to mixed orbitals, as discussed in Sec. VI.E.1. It should be stressed that the range $168.2-169.4 \mathrm{eV}$ includes some $\left(2 \mathrm{p}_{3} / 2\right)^{-1}$ core-excited Rydberg states as well as the $\left(2 \mathrm{p}_{1 / 2}\right)^{-1}$ core-excited mixed states. Deconvoluting these different contributions to the spectra in this region and in other regions is one of the main accomplishments of the least-squares analysis.

Without a detailed assignment of the mixed-state transitions for both isotopic species, a reliable one-to-one correlation of the mixed states between $\mathrm{H}_{2} \mathrm{~S}$ and $\mathrm{D}_{2} \mathrm{~S}$ is not available because of their large isotopic differences. As a result, assumption (4) was not applied to the mixed states; the intensity ratios used for these peaks are not the sarne for $\mathrm{H}_{2} \mathrm{~S}$ and $\mathrm{D}_{2} \mathrm{~S}$ (see Table VI-2). In order to obtain a good fit to the data, it was also necessary to relax assumption (1) for the mixed states. The mean spin-ortit splitting and molecular-field splitting used for all of the mixed states were allowed to differ from the splittings used for the rest of the Rydberg spectrum, and the overall fit improved dramatically for both $\mathrm{H}_{2} \mathrm{~S}$ and $\mathrm{D}_{2} \mathrm{~S}$. This result, along with observation of extensive vibrational excitations, is the main motivation for identifying the states in Table VI-2 as intermediate states. The sharp distinction between the mixed and Rydberg states assumed 
for this analysis is, of course, only an approximation. A more realistic model would allow the splittings of each state to be unique, and the resulting variation of the energy splittings observed would probably not be as abrupt.

For the mixed states, the least-squares analysis resulted in a molecular-field splitting of $39(9) \mathrm{meV}$ and a mean spin-orbit splitting of $1.112(6) \mathrm{eV}$. For each quantity, the estimated uncertainty of the last digit is given in parenthesis. For the Rydberg states, the analysis gave a molecular-field splitting of $115(9) \mathrm{meV}$ and a mean spin-orbit splitting of $1.204(6) \mathrm{eV}$. Thus, the molecular-field splitting is $76 \mathrm{meV}$ smaller and the mean spin-orbit splitting is $92 \mathrm{meV}$ smaller in the mixed states, as compared to the Rydberg states. These modified splittings can occur if the direct and/or exchange interactions with the sulfur core hole are stronger for an electron in an intermediate-type ortital than they are for an electron in a Rydberg orbital. The splittings for the Rydberg states were mainly determined in the fit by the intense features assigned in Table VI-1 to excitations of 3d-and 4d-derived Rydberg states. They are probably also fairly accurate for the higher Rydherg states. Even these near-threshold splittings may include some direct-and exchange-interaction contributions, but they nonetheless can be compared to recent measurements made by Svensson, et .al., using high-resolution XPS and AES [7). In that letter, a small difference was reported between the spin-orbit splitting of the $\mathrm{H}_{2} \mathrm{~S}$ sulfur $2 \mathrm{p}$ level as measured by XPS and by AES. This results were explained, in part, by estimating the molecular-field splitting of the sulfur $2 p_{3 / 2}$ level to be $=106 \mathrm{meV}$ in the core-excited ion. The mean spinorbit splitting measured by XPS was $1.201 \mathrm{eV}$. Both of these values are in very good agreement with the corresponding quantities measured in the present experiment for the core-excited Rydberg states, suggesting that the interpretation of Svensson, et al., is valid and also that Coulombic interactions do not affect the measured splittings very much for the Rydberg states.

As a test, an alternative least-squares analysis of the data was performed with the molecular-field splitting fixed at zero. Otherwise this "no-splitting" test analysis was 
subject to exactly the same freedoms and restrictions as the "split" fit. Thus the "split" fit had only wo more adjustable parameters than the "no-splitting" nit; the 2p3/2 splitting of the Rydberg states and of the mixed states. The results of the "no-splitting" fit, shown in Fig. VI-5, are not as convincing as the results of the "split" fit presented above and in Fig. VI-2. Most notably, the regions around 1 (19.0-169.4 $\mathrm{eV}$ and $169.8-170.2 \mathrm{eV}$ are poorly approximated by the "no-splitting" fit. Besides giving a poorer fit, the derived parameters are more difficult to interpret. The intensity ratios, $X$, for the more intense features show significantly larger deviations from unity in the "no-splitting" fit than in the "split" fit presented here in Tables I and II. For example, in the "no-splitting" fit for $\mathrm{H}_{2} \mathrm{~S}\left(\mathrm{D}_{2} \mathrm{~S}\right)$, five of the ten most intense features have a ratio outside the range 0.7-2.4 (0.5-2.4). By comparison, in the "split" fit for $\mathrm{H}_{2} \mathrm{~S}\left(\mathrm{D}_{2} \mathrm{~S}\right)$, only one (Iwo) of the ten most intense features have a ratio outside the narrower ranges $0.7-1.2$ (0.7-1.5). As mentioned above, some deviation of the intensity ratio, $X$, from unity is plausible, but when ratios for many intense peaks assume values much smaller or larger than one, with no apparent pattem, the validity of the analysis is questionable. The results of the "no-splitting" fit are much poorer than would he expected merely from reducing the total number of adjustable parameters by two, as compared to the "split" fit. This test therefore provides substantial evidence for the molecular-field splitting of the $2 p_{3 / 2}$ core level. The unambiguous identification of the $2 p_{3 / 2}$ molecular-field splitting in this spectrum is, in fact, the most significant result of this work.

Just helow threshold, the Rydberg formula successfully predicts the energies of the transitions, as discussed in Sec. VI.E.2. The quantum defects obtained from this analysis can be used to roughly characterize the lower-energy core-excited states. Using the quantum defect determined from the energies of the $\left(2 p_{1 / 2}\right)^{-1}$ nd $(n=5-8)$ Rydherg states. the term value of the $2 \mathrm{p}^{-1} 3 \mathrm{~d}$ state is predicted to be $1.89 \mathrm{eV}$. As shown in Table VI-1, there are several intense transitions with term values close to this prediction. They have therefore been assigned as "3d-derived"; i.e. they arise from several molecular core-excited 
states which can be associated with the atomic $2 p^{-1} 3 \mathrm{~d}$ stale. Similarly, there are several 4d-derived states (see Sec. VI.E.2), hut they are spread over a narrower energy range as compared to the $3 \mathrm{~d}$-derived states, because they have a larger spatial extent and are influenced less by the molecular fleld.

Based upon the energy of the $\left(2 p_{1 / 2}\right)^{-1}$ 6s state, the 58 state should have a term value of $-1.6 \mathrm{cV}$. A medium-intensity feature with this term value has been tentatively assigned to a 5s-derived stale, as indicated in Table VI-1 and Fig. VI-2. The 48 state is

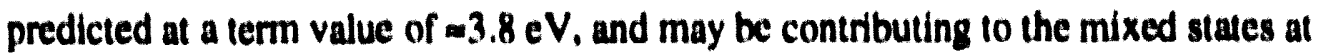
somewhat lower term values and/or to the valence-shell states at somewhat higher ierm values. The defect determined from the energies of the $2 p^{-1} n p(n=6-8)$ Rydherg states predicts a term value of $2.43 \mathrm{eV}$ for the $2 \mathrm{p}^{-1} 4 \mathrm{p}$ states. Tahle VI.2 shows that the spectra of both $\mathrm{H}_{2} \mathrm{~S}$ and $\mathrm{D}_{2} \mathrm{~S}$ have hands centered at slightly higher tenn values (lower transition energies). These bands include significant vibrational excitations, so the average fundamental term values of the electronic states must be even higher than the band center. This is consistent with the assignment of this band to mixed states, hecause the valence contributions to these states could shift them to higher term values than expected for pure 4p-derived Rydhery states. It should be stressed that, for the stales in these spectra with term values greater than $=1 \mathrm{eV}$, the association with a specific alomic Rydberg state is approximate at best. In fact, states which share the same total symmetry and similar energies can $\mathrm{mix}$, and thus a single core-excited state may be derived from a combination of atomic s-, p-, and d-states. Indeed, it is the mixing of d-character into the p-states which gives the p-series greater intensity than the s-series in the near-lhreshold region [34].

\section{Comparison to other XANES spectra}

\section{a. Mixed states}


A comparison to related XANES spectra is especially useful for the interpretation of the splittings of the mixed states in $\mathrm{H}_{2} \mathrm{~S}$. The sulfur $\mathrm{L}_{2,3}$ photoionization spectrum of $\mathrm{SF}_{6}$ shows intense transitions to the $(S 2 p)^{-1} a_{1 g}$ valence-shell states as well as much weaker transitions to (S 2p) $)^{-1}$ Rydberg states (see Sections V.D and V.E). The Rydberg states with the largest term value. $(S 2 p)^{-1} 4 s$, showed the same spin-orbit splitting as the higher Rydberg states, weak vibrational excitations, and only a small deviation from the energy predicted by the quantum defect of the higher ns states. It therefore appears that there is relatively little valence-Rydberg mixing in the $(S 2 p)^{-1}$ states of $\mathrm{SF}_{6}$, presumably because the large potential harrier prevents the interaction of valence and Rydherg orbitals. This is in distinct contrast to $\mathrm{H}_{2} \mathrm{~S}$, where the hydrogen atoms are not expected to create an appreciable potential barrier, and transitions to mixed stales are observed.

The comparison of the $(S 2 p)^{-1}$ Rydherg states and the $(S 2 p)^{-1}$ alg valence-shell states in $\mathrm{SF}_{6}$ is particularly relevant here. A slightly smaller spin-orbit splitting was measured for the valence-shell states, as compared to the splitting of the Rydherg states. Note that molecular-field splitting of the sulfur $2 p$ levels is not possible in SF 6 because of the octahedral symmetry of that molecule. Therefore the best explanation for this apparent reduction of the spin-ortit splitting is the following: hecause of increased spatial overlap. the exchange interaction of the excited electron with the core hole is larger in the valenceshell states than in the Rydherg states, and the dependence of that interaction on the j-value of the core hole produces the observed reduction in the spin-orbit splitting (see Section V.D.1). The observed shift of $\mathrm{m.30} \mathrm{meV}$ in $\mathrm{SF}_{6}$ is smaller than the effect observed in $\mathrm{H}_{2} \mathrm{~S}$ for the mean spin-orbit splitting of the intermediate states (shift $=-92 \mathrm{meV}$ ). As mentioned above in Sec. VI.D, the exchange interaction in SF ${ }_{6}$ for the valence-shell states has a much greater effect upon the intensity ratio of the two $\left(S_{2} 2 p_{1 / 2,3 / 2}\right)^{-1} a_{g}$ transitions than it does upon the spin-ortit splitting. Similar behavior is also seen in spectra of the xenon fluorides, and was predicted by calculations [25]. The measured ratio in $\mathrm{SF}_{6}$ was 0,62 $\left(2 p_{3 / 2}: 2 p_{1 / 2}\right)$ for the valence-shell states as compared to 2.07 for the Rydherg states $|35|$. 
Note that the statistical ratio, expected in the limit of no exchange interaction, is 2.00 , corresponding to an intensity ratio of $X=1.00$ in the notation used here for $\mathrm{H}_{2} \mathrm{~S}$.) Summing the intensities of all the mixed states, the overall intensity ratio $1.03(1.04)$ is obtained for $\mathrm{H}_{2} \mathrm{~S}\left(\mathrm{D}_{2} \mathrm{~S}\right)$. For the summed Rydberg states, the overall intensity ratio is 1.03 (1.01) for $\mathrm{H}_{2} \mathrm{~S}\left(\mathrm{D}_{2} \mathrm{~S}\right)$. These ratios are estimated to be accurate to within $\approx 5 \%$, desplte the limitations of the analysis.

The behavior of the mixed states in $\mathrm{H}_{2} \mathrm{~S}$ is thus quite different from that of the valence-shell states in $\mathrm{SF}_{6}$ : the mean spin-oroit splitting changes significantly from the Rydberg value but the intensity ratio deviates very little from the statistical value. This suggests that the exchange interaction is not the primary cause of the change in mean spinorbit splitting in the mixed states. The difference can also result from the lower symmetry of $\mathrm{H}_{2} \mathrm{~S}$, which allows the observed mean spin-orbit splitting to have contributions from the direct Coulomb interaction of the excited electron with the core hole; this is not possible in the higher-symmetry SF 6 molecule. Therefore much of the $92 \mathrm{meV}$ reduction in the observed mean spin-ortit splitting for the mixed states of $\mathrm{H}_{2} \mathrm{~S}$ can be tentatively attributed to differences in the direct interaction of the excited electron with the different core holes. An accurate theoretical treatment which includes all possible effects is necessary for a more definite understanding of the mixed states.

\section{b. Rydberg states}

A number of earlier works have studied the $\mathrm{L}_{2,3}$ spectra of third-row hydrides $\mathrm{SiH}_{4}$ $[3,4,5,36,37], \mathrm{PH}_{3}[3,4,5,12,36,38,39], \mathrm{H}_{2} \mathrm{~S}[2,3,4,5], \mathrm{HCl}[3,4,5,13,40)$, and the isoelectronic atom, $\operatorname{Ar}[29,30]$. There is still some uncertainty concerning the assignments of the various spectra. A complete consideration of these matters is beyond the scope of this analysis, but some discussion is necessary. Overall, the most important guiding principle for assignment is the similarity of term values for the atomic-like outermost 
Rydberg orbitals in all these systems. Thus, the spectrum of argon guides the assignment for the upper regions of all the spectra.

The high-resolution $\mathrm{L}_{2,3}$ spectrum $0_{i} \mathrm{Ar}$, as measured by EELS, was thoroughly discussed in Ref. [30]. For the present analysis, a high-resolution XANES spectrum [29] with better a signal-to-noise ratio than the EELS spectrum was fitted using a similar assignment. The quantum defects obtained are given in Table VI-3. Due to the accidental equality of $\delta_{\mathrm{s}}$ and $\delta_{\mathrm{d}}$, within modulus 2 , each $2 \mathrm{p}^{-1} \mathrm{~ns}$ Rydberg state above $\mathrm{n}=4$ is exactly degenerate with the corresponding $2 \mathrm{p}^{-1}(\mathrm{n}-2) \mathrm{d}$ state. Calculations in Ref. [41] predict, however, that the transitions to the (n-2)d states account for $\approx 95 \%$ of the obset ved intensity, as compared to the overlapping ns states. As a result of the multipolar nature of EELS excitations, the dipole-forbidden transition to the $\left(2 p_{3 / 2}\right)^{-1} 4 p$ state was also observed in Ref. [30], and the derived quantum defect is also given in Table VI-3. This highlights one perhaps surprising aspect of the assignments for the upper $(2 p)^{-1}$ Rydberg states given in Sec. VI.E.2 and Table VI-1: the intensities of transitions to the 5p and 6p orbitals are comparable to those for the $5 \mathrm{~s}$ and $6 \mathrm{~s}$ orbitals, even though, in atomic dipoleselection rules, the $2 p$ to np transition is dipole-forbidden. Since higher Rydberg orbitals are increasingly atomic-like, it might be expected that these transitions would be very weak in $\mathrm{H}_{2} \mathrm{~S}$. As discussed in Section I.D.1, although these higher orbitals are atomic-like over most of their spatial distribution, the region of the Rydberg orbital which overlaps the core electron is within the molecule and feels the full molecular field. Thus, the molecular dipole selection rules apply even for transitions to the higher Rydberg states. For $\mathrm{H}_{2} \mathrm{~S}$, calculations have shown the mechanism of this effect [34]: Because of the non-spherical molecular symmetry, the p orbitals mix with the $d$ orbitals and "borrow" intensity from the very favorable atomic $2 \mathrm{p} \rightarrow \mathrm{nd}$ transitions.

For the $\mathrm{L}_{2,3} \mathrm{HCl}$ spectrum, the assignment of Ref. [40] best follows the pattern observed in argon, i.e. a dominant $2 \mathrm{p}^{-1}$ nd-series, with contributions from the $2 \mathrm{p}^{-1} \mathrm{~ns}$ states. Due to the reduced symmetry, as compared to Ar, transitions to $2 \mathrm{p}^{-1} \mathrm{np}$ states are 
dipole-allowed, and the $2 \mathrm{p}^{-1} 4 \mathrm{p}$-derived excitation is fairly intense. For $\mathrm{PH}_{3}$, the spectrum in Ref. [12] has the best available resolution and statistics, but the assignment given for the Rydberg states is not entirely convincing. In fact, the uppermost region of the spectrum in Ref. [12] (term values less than $\approx 1.3 \mathrm{eV}$ ) is remarkably similar to same region of the $\mathrm{H}_{2} \mathrm{~S}$ spectrum presented here. This resemblance strongly suggests that the assignment of all structure in this region to vibrational sidebands, as suggested for $\mathrm{PH}_{3}$ in Ref. [39], is also incorrect. Applying the present $\mathrm{H}_{2} \mathrm{~S}$ assignment to the term values derived in Ref. [12] gives the quantum defects for $\mathrm{PH}_{3}$ shown in Table VI-3. Recent measurements with high resolution for $\mathrm{SiH}_{4}$ gave the quantum defects shown in Table VI-3 [37]. Note that the dseries in $\mathrm{SiH}_{4}$ is strongly split by the molecular field, and also that the quantum defects given in Ref. [37] for the two resulting d-series were reduced by one in Table VI-3. Overall, the comparison in Table VI-3 shows that the values of $\delta$ derived for $\mathrm{H}_{2} \mathrm{~S}$ are consistent with the corresponding quantum defects in closely related spectra.

\section{Comparison to previous $\mathrm{H}_{2} \mathrm{~S}$ results}

The $\mathrm{L}_{2,3}$ near-edge spectrum for $\mathrm{H}_{2} \mathrm{~S}$ shown here is significantly improved over earlier published spectra. Recently a spectrum was published [2] with resolution nearly as good as in the present case, but with a poorer signal-to-noise ratio, and no attempt was made to assign the peaks or analyze the spectrum. The first experimental results were presented by Hayes and Brown [3]. Due to limited resolution, only the general outline of the spectrum was seen, without the extensive fine structure now observed. The initial assignments of Hayes and Brown were later re-assessed by Schwarz [4] and Robin [5]. Both of these later assignments attributed the broad features at lowest energies to valenceshell excitations. The higher-energy features were assigned to s-, p-, and d-Rydberg states. Schwarz, in particular, assigned all observed features in a manner consistent with his assignments for the corresponding spectra of other third-row hydrides. The present 
assignments, made in the light of considerably more resolved peaks and a detailed analysis, agree with Schwarz in the assignment of the $6 a_{1}, 3 b_{2}$, and d-Rydberg states. Schwarz's $5 s$ and $6 \mathrm{~s}$ states are attributed here to p-type states, resulting in consistent quantum defects as discussed in the preceding section. The $4 \mathrm{~s}$ and $4 \mathrm{p}$ states are not explicitly assigned here, because the strong influence of the molecular field makes these atomic designations inapplicable. However the mixed states identified here can be loosely attributed to $4 \mathrm{p}$ and $4 s$ atomic parentage (see Sec. VI.E.3.b), which is then in agreement with Schwarz's assignment.

\section{Vibrational structure}

A direct comparison of the $\mathrm{H}_{2} \mathrm{~S}$ and $\mathrm{D}_{2} \mathrm{~S}$ spectra in Fig. VI-2 identifies the features which arise from vibrational excitations, because of the isotopic dependence of vibrational spacings. The least-squares analysis of the spectra essentially deconvolutes the effects of the core-level splittings. The term values obtained are listed in Tables I and II; differences between those of $\mathrm{H}_{2} \mathrm{~S}$ and $\mathrm{D}_{2} \mathrm{~S}$ may be attributed to vibrational sidebands. Weak excitations of the symmetric stretch mode were observed in photoelectron spectra from the $2 p$ levels, with a branching ratio of $\approx 5 \%$ for the first vibrational peak [9]. Similar excitations are expected for the higher Rydberg states in these measurements. No such excitation was observed, however, perhaps because of the small branching ratio. As mentioned in Sec. VI.E.2, the $\left(2 \mathrm{p}_{1 / 2}\right)^{-1} 5 \mathrm{p}, 4 \mathrm{~d}$ region shows a small vibrational sideband probably associated with the $v_{2}$ bend mode.

The peak at $\approx 169.55 \mathrm{eV}$ has noticeably larger intensity in the $\mathrm{D}_{2} \mathrm{~S}$ spectrum, but the results of the analysis do not suggest any simple vibrational explanation. Those results may be somewhat inaccurate considering the unusually small intensity ratio $(X=0.2)$ obtained for one of the two states contributing to this $3 \mathrm{~d}$-derived feature, as well as the small intensity ratios for the states at $168.37 \mathrm{eV}$ which overlap the $\left(2 \mathrm{p}_{3} / 2\right)^{-1}$ states of this 
3d-derived feature.

The mixed states show the most obvious isotopic effects in the spectra. The derived term values in Table VI-2 show some regular spacing, indicated by the labels A1, $\mathrm{A}$, etc. For $\mathrm{H}_{2} \mathrm{~S}$, a spacing of $\approx 155 \mathrm{meV}$ is apparent from the results of the fit, presumably due to a vibrational progression in the $v_{2}$ bend mode, which has a ground state spacing of $147 \mathrm{meV}$ [33]. Regular spacings are much less evident in the $\mathrm{D}_{2} \mathrm{~S}$ results, but a spacing of $140 \mathrm{meV}$ is observed, possibly corresponding to the $v_{2}$ bend mode, which has a ground state spacing of $106 \mathrm{meV}$ [33]. For neither molecule is there a simple way to assign all the states to vibrational progressions of a single electronic transition. Instead, there seems to be at least two electronic transitions in this energy range. The difficulty in interpreting the spectra even as several overlapping vibrational progressions could arise from strong vibronic coupling between two or more electronic states. These interactions can produce irregular fine structure in absorption spectra [42].

\section{VI.F. Sulfur $L_{2,3}$ edges: Valence shell excitations}

\section{Assignment and electron-hole interaction}

The broad, overlapping peaks shown in Fig. VI-3 are assigned to one-electron excitations from the sulfur $2 p$ core levels to the $6 a_{1}$ and $3 b_{2}$ molecular orbitals, in agreement with previous reports [4,5]. A recent calculation predicted the $3 b_{2}$ orbital to have lower energy than $6 a_{1}$ in the presence of a $2 p$ core hole [43]. The opposite order $6 a_{1}$ $<3 b_{2}$ calculated in Refs. $[4,15,18]$ will be assumed here. A simple extension of the discussion in Sec. VI.E.1 shows that, in $\mathrm{H}_{2} \mathrm{~S}, 18$ dipole-allowed final states result from the combination of two unoccupied molecular orbitals with the three possible core levels which may excite them. In fact, as seen above for the Rydberg states, many of these electronic states are nearly degenerate. 
As discussed above in Sec. VI.E.4.a, the spin-orbit splittings and intensity ratios of core-excited valence-shell states are expected to show the effect of the exchange interaction (and possibly the direct interaction) between the core hole and excited electron. In an effort to characterize these effects in the spectra of Fig. VI-3, approximate fits were made to the data. The features between 163 and $167 \mathrm{eV}$ were modeled by two doublets, with splittings arbitrarily set equal, described by four very broad Gaussian peaks. The Gaussian lineshape roughly approximates the non-Lorentzian broadening due to unresolved or barely-resolved vibrational progressions. The four peaks represent the states, in order of

increasing energy, which roughly account for the actual spectrum: $\left(2 \mathrm{p}_{3 / 2}\right)^{-1} 6 \mathrm{a}_{1},\left(2 \mathrm{p}_{3 / 2}\right)^{-1}$ $3 b_{2},\left(2 p_{1 / 2}\right)^{-1} 6 a_{1}$, and $\left(2 p_{1 / 2}\right)^{-1} 3 b_{2}$.

A least-squares analysis based on this simplified model gave the results shown in Table VI-4. The spin-orbir splitting values are slightly smaller than the mean spin-orbit splitting determined for the mixed states and significantly smaller than the mean spin-orbit splitting determined for the Rydberg states. This supports the expectation that Coulombic interactions between the core hole and excited electron are most significant for the coreexcited valence-shell states, less so for the core-excited mixed states, and least important for the core-excited Rydberg states. The derived intensity ratios for the $3 b_{2}$ excitations are much less than the statistical value (2.00), indicating that Coulombic interactions are largest for those states. Similar deviations of the intensity ratio from the statistical value have been observed in the corresponding core-excited valence-shell states of other third-row hydrides $[4,36]$. While the quantitative results of this simplified analysis may be unreliable, the qualitative trends in splittings and intensity observed here very likely reflect real effects.

\section{Vibrational structure and other isotopic shifts}

The spectra of Fig. VI-3 show some regularly-spaced fine structure, more easily observed in the expanded insets, with a notable isotopic dependence. A comparison of the 
two spectra also reveals small differences in the overall lineshape and peak position. The dissociative nature of the electronic states must be considered in the interpretation of these results. Recent photo-ion [16] and Auger electron [17,18] studies have shown that rapid predissociation is the dominant decay process for these core-excited valence-shell states. The core-excited molecule fragments into $\mathrm{H}$ and $\mathrm{HS}^{*}$ with a rate faster than the Auger decay of the $2 \mathrm{p}$ core hole. There has been considerable interest in lower-energy dissociative electronic states of $\mathrm{H}_{2} \mathrm{~S}[44,45,46]$. Specifically, the broad peak in the UV absorption spectrum centered at $\approx 195 \mathrm{~nm}$ is known to correspond to a dissociating state. [45]. This peak shows isotopic-dependent weak fine structure which is notably similar to the structure of Fig. VI-3. This similarity may result from analogous electron configurations; the $195 \mathrm{~nm}$ band corresponds to the excitation of the outermost groundstate electron, in the non-bonding $2 b_{1}$ orbital, to the $6 a_{1}$ orbital. The transition to the $3 b_{2}$ orbital is dipole-forbidden, but that excited state strongly influences the observed spectrum through vibronic coupling [46].

A comparison of the two spectra of Fig. VI-3 shows a shift of the leading edge of the peak to higher energy in $\mathrm{D}_{2} \mathrm{~S}$. Both the peak maximum and the trailing edge show a shift to lower energy in $\mathrm{D}_{2} \mathrm{~S}$. To illustrate these shifts, Fig. VI-3 indicates the position of the peak maxima and the leading- and trailing-edge inflection points with vertical lines. The shift of the leading edge is expected for a direct transition to a dissociative electronic state, because of zero-point vibrational energy effects (see Section I.D.6). The potential surface of such an excited state does not support vibrational states for the asymmetric-stretch mode, and there is no contribution to the total energy of the excited state from the zero-point energy of this mode. The zero-point energy of this mode does contribute to the energy of the ground state, and that contribution is half the harmonic vibrational energy. The harmonic energies are $337 \mathrm{meV}$ for $\mathrm{H}_{2} \mathrm{~S}$ and $\approx 249 \mathrm{meV}$ for $\mathrm{D}_{2} \mathrm{~S}$ [33]. The observed transition energy to a dissociative electronic state should therefore be $1 / 2(337-249)=44$ meV larger for $\mathrm{D}_{2} \mathrm{~S}$. This agrees with the observed shift of $+0.05(2) \mathrm{eV}$ for the leading- 
edge inflection point in $\mathrm{D}_{2} \mathrm{~S}$ as compared to $\mathrm{H}_{2} \mathrm{~S}$. The leading-edge region of the peak is dominated by transitions to the $\left(2 \mathrm{p}_{3 / 2}\right)^{-1} 6 \mathrm{a}_{1}$ state, which was calculated to be dissociative by Naves de Brito, et. al. [18]. It should be noted that changes in the spacings of the other two vibrational modes upon excitation also have an effect on transition energies. These zero-point differences may also contribute to the observed shift.

A series of Voigt functions were used in a least-squares analysis to fit the weak fine structure observed in the range $\approx 165.0-165.7 \mathrm{eV}$ for both $\mathrm{H}_{2} \mathrm{~S}$ and $\mathrm{D}_{2} \mathrm{~S}$. The results of this analysis are plotted with the data in the expanded portion of Fig. VI-3 and listed in Table VI-5. In principle, the individual peaks barely resolved in the spectra could be vibrational sidebands of more than one electronic transition. In the region of the vibrational structure the strongest contributions are probably from the $\left(2 p_{3 / 2}\right)^{-1} 3 b_{2}$ state. Weaker contributions from the $\left(2 \mathrm{p}_{1 / 2}\right)^{-1} 6 \mathrm{a}_{1}$ and $\left(2 \mathrm{p}_{3 / 2}\right)^{-1} 6 \mathrm{a}_{1}$ states are also possible. These designations are only approximate; as many as fifteen distinct electronic states could in fact contribute in this range. Assuming that the sidebands are a single progression from only one electronic transition gives reasonable results, however. This suggests that much of the possible electronic splitting is not actually large enough to affect the spectrum. In particular, the molecular-field splitting of the $2 \mathrm{p}_{3 / 2}$ level must be less than $\approx 50 \mathrm{meV}$, notably smaller than the value derived for the upper Rydberg states. The average spacing determined for the peaks in this range is $167(3) \mathrm{meV}$ for $\mathrm{H}_{2} \mathrm{~S}$ and $145(3) \mathrm{meV}$ for $\mathrm{D}_{2} \mathrm{~S}$.

The interpretation of an apparent vibrational progression in the excitation of a dissociative state is especially difficult in the present case where vibronic coupling may have a significant influence. Based upon the following arguments, the observed progression is tentatively assigned to excitation of the $v_{1}$ symmetric-stretch mode of the $\left(2 p_{3 / 2}\right)^{-1} 3 b_{2}$ state. The calculations of the $(2 p)^{-1} 6 a_{1}$ and $(2 p)^{-1} 3 b_{2}$ hypersurfaces by Naves de Brito, et. al., [18] are particularly useful. They found that the $(2 p)^{-1} 3 b_{2}$ excited state, at higher energy, is bound, but strongly vibronically-coupled to the dissociative (2p)$16 a_{1}$ state. Assuming this model, there are two possible origins for the observed 
vibrational structure: (1) Excitation of the dissociative (2p)-1 $6 a_{1}$ state may exhibit a diffuse vibrational progression in a non-dissociative mode [47]. (2) The vibrational states may reside on the bound $(2 p)^{-1} 3 b_{2}$ potential, before the system crosses to the dissociative potential. The latter explanation, for lower-energy states, was recently obtained by Schinke and coworkers from calculations of the fine structure of the $195 \mathrm{~nm}$ band of $\mathrm{H}_{2} \mathrm{~S}$ [46]. They attribute the observed progression to excitation of the $v_{1}$ symmetric-stretch mode in a bound state, which is strongly vibronically-coupled to a dissociative state at lower energy. This result is somewhat unexpected, because the observed spacings in the $195 \mathrm{~nm}$ band of $\mathrm{H}_{2} \mathrm{~S}$ and $\mathrm{D}_{2} \mathrm{~S}$ are almost exactly equal to the corresponding $v_{2}$ symmetric-bend mode spacings of the ground state.

Based only on their derived spacings in Table VI-5, the peaks of Fig. VI-3 might be attributed to a progression in the $v_{2}$ bending mode, which has a spacing of $147 \mathrm{meV}$ and $106 \mathrm{meV}$ in the ground state of $\mathrm{H}_{2} \mathrm{~S}$ and $\mathrm{D}_{2} \mathrm{~S}$, respectively [33]. The results of Schinke and coworkers [46] suggest the alternate possibility that the progression is associated with a much-softened $v_{1}$ symmetric-stretch mode, which has a spacing of $324 \mathrm{meV}$ and 235 $\mathrm{meV}$ in the ground state of $\mathrm{H}_{2} \mathrm{~S}$ and $\mathrm{D}_{2} \mathrm{~S}$, respectively [33]. Indeed, the bound $(2 \mathrm{p})^{-1} 3 \mathrm{~b}_{2}$ potential calculated by Naves de Brito, et. al., [18] appears to have a much smaller slope (i.e. smaller force constant) in the direction of the symmetric stretch as compared to the calculated ground state potential. Thus the vibrational progression observed here is tentatively assigned to the $v_{1}$ symmetric-stretch mode of the $\left(2 p_{3 / 2}\right)^{-1} 3 b_{2}$ state. A corresponding progression of the $\left(2 \mathrm{p}_{1 / 2}\right)^{-1} 3 \mathrm{~b}_{2}$ state was not clearly resolved, but a secondderivative analysis of the spectra identified some regularly-spaced fine structure in the range $\approx 166.3-166.9 \mathrm{eV}$, with a spacing of $\approx 154 \mathrm{meV}(\approx 127 \mathrm{meV})$ for $\mathrm{H}_{2} \mathrm{~S}\left(\mathrm{D}_{2} \mathrm{~S}\right)$. The lack of distinct fine structure for the $\left(2 \mathrm{p}_{1 / 2}\right)^{-1} 3 \mathrm{~b}_{2}$ state may reflect a larger natural linewidth than for the $\left(2 \mathrm{p}_{3} / 2\right)^{-1} 3 \mathrm{~b}_{2}$ state, perhaps because this higher-energy state has access to additional decay channels. The ratio of vibrational spacings in the $\left(2 \mathrm{p}_{3} / 2\right)^{-1} 3 \mathrm{~b}_{2}$ excited state is $0.87(3)\left(\mathrm{D}_{2} \mathrm{~S}: \mathrm{H}_{2} \mathrm{~S}\right)$, as compared to 0.72 in the ground state for the $v_{1}$ mode (and the $v_{2}$ 
mode). This difference indicates an isotopic dependence of the potential surfaces. This could result from the strong vibronic coupling between the electronic states predicted by Naves de Brito, et. al., for the region of the vertical transition.

Portions of the spectra in Fig. VI-3 are attributed to the excitations of bound states, which should be accompanied by progressions of vibrational excitations. The resulting contributions to the observed spectrum are broad bands, each composed of a series of individual vibronic peaks. Therefore, the center of such a band should shift to lower energies for $D_{2} S$, because of the smaller vibrational spacings as compared to $\mathrm{H}_{2} \mathrm{~S}$. This explains the shifts of the peak maximum and the trailing-edge inflection point indicated in Fig. VI-3, which are $-0.08(1) \mathrm{eV}$ and $-0.05(3) \mathrm{eV}$ (for $\mathrm{D}_{2} \mathrm{~S}$ as compared to $\mathrm{H}_{2} \mathrm{~S}$ ) and are associated with the $\left(2 n_{3 / 2}\right)^{-1} 3 b_{2}$ and $\left(2 p_{1 / 2}\right)^{-1} 3 b_{2}$ states, respectively. These shifts may also include contributions from the vibrational zero-point energy changes described above. which are expected to be positive and less than $\approx .05 \mathrm{eV}$ in this case.

\section{VI.G. Linewidths}

The least-squares fit presented in Table VI-5 and in Fig. VI-3 gave an excellent fit to the fine structure of the valence-shell states, suggesting that the derived parameters have physical significance. Assuming an instrumental linewidth of $30 \mathrm{meV}$ (Gaussian FWHM), the derived natural linewidths (Lorentzian FWHM) for the individual vibrational peaks in the fit are 344 (15) meV for $\mathrm{H}_{2} \mathrm{~S}$ and $306(15) \mathrm{meV}$ for $\mathrm{D}_{2} \mathrm{~S}$. The implication is that these core-excited valence-shell states decay faster for $\mathrm{H}_{2} \mathrm{~S}$ than for $\mathrm{D}_{2} \mathrm{~S}$, an isotopic dependence which is unexpected for the Auger decay process. This result is consistent with the assignment of this fine structure to vibrations of a bound state, which is rapidly depopulated by vibronic coupling to a dissociative state. The observed isotopic lifetime dependence indicates that the vibronic coupling is somewhat weaker for $\mathrm{D}_{2} \mathrm{~S}$.

These valence-shell excitation natural linewidths are much larger than those derived 
for the Rydberg excitations. For example, assuming an instrumental linewidth of $30 \mathrm{meV}$, the derived natural linewidths for $\mathrm{H}_{2} \mathrm{~S}$ are $65 \mathrm{meV}$ and $55 \mathrm{meV}$ for the $\left(2 \mathrm{p}_{1 / 2}\right)^{-1} 7 \mathrm{~d}$ and $\left(2 p_{1 / 2}\right)^{-1} 8 d$ states, respectively, compared with $344 \mathrm{meV}$ for the valence-shell states. This trend is consistent with the decreasing evidence of predissociation observed by photo-ion spectroscopy as the excitation energy was increased into the Rydberg region of the photoabsorption spectrum [16]. A similar difference in dertved natural linewidths is observed in the corresponding sulfur $\mathbf{L}_{2,3}$ spectrum of $\mathrm{SF}_{6}$, where predissociation is not expected to occur (see Section V.E.3). In that case, the differences are interpreted as the result of a valence-shell-excited electron participating much more strongly in the Auger decay than a Rydherg-excited electron. For $\mathrm{H}_{2} \mathrm{~S}$, the predissociation of the valence-shell excitations precludes a direct comparison to the corresponding states of $\mathrm{SF}_{6}$. The derived natural linewidths of the Rydberg excitations may be compared, however. The narrowest natural linewidth for $\mathrm{H}_{2} \mathrm{~S}$ is $55 \mathrm{meV}$, compared to $35 \mathrm{meV}$ in $\mathrm{SF}_{6}$. The difference reflects the effect of chemical substituents on the electron density at the sulfur core: six fluorine atoms will withdraw much more electron density then two hydrogen atoms, resulting in a much-reduced rale of Auger decay. The calculated natural linewidth for atomic sulfur is $\mathbf{5 4}$ meV [22], very close to the minimum value measured for $\mathrm{H}_{2} \mathrm{~S}$.

\section{VI.H. Sulfur $L_{1}$ Edge}

The high-resolution photoionization spectra of $\mathrm{H}_{2} \mathrm{~S}$ and $\mathrm{D}_{2} \mathrm{~S}$ near the sulfur $\mathrm{L}_{1}$ edge are shown in Fig. VI-4. Apparently, XANES spectra in this range have not been measured previously for hydrogen sulfide. Several resonances are observed in each spectrum, leading up to an edge jump. The interpretation of the spectrum is facilitated by comparison to the very similar XANES spectrum of $\mathrm{H}_{2} \mathrm{~S}$ at the sulfur $\mathrm{K}$ edge [8]. With those results as a guide, the most intense resonance, at $228.9 \mathrm{eV}$, is assigned to overlapping transitions to the $3 \mathrm{~b}_{2}$ and $6 \mathrm{a}_{1}$ valence-shell orbitals. The next feature, at 232.0 
eV, is attributed to transitions to $4 \mathrm{p}$-derived and/or $3 \mathrm{~d}$-derived orbitals. The weak feature at $233.4 \mathrm{eV}$, which is more clearly seen in the lower-noise $\mathrm{D}_{2} \mathrm{~S}$ spectrum, is assigned to overlapping higher Rydberg states. The spectra of $\mathrm{H}_{2} \mathrm{~S}$ and $\mathrm{D}_{2} \mathrm{~S}$ are very similar, but there may be slight differences in the valence-shell resonances. In particular, there appears to be a shift of the leading edge of the peak to higher energy in $D_{2} S$, as also seen for the valence. shell peak below the $\mathrm{L}_{2,3}$ edges. This shift is $=0.1 \mathrm{eV}$, as mensured by the change in leadingedge inflection point, with the spectra aligned at the $231.5 \mathrm{eV}$ peak. Furthermore. there are slight indications of vibrational fine structure in the valence-shell resonances (see insets of Fig. VI-4). Although the present results are not completely convincing, measurements with better resolution and counting rates might resolve structure similar to that seen in Fig. VI-3 for the (2p)-1 valence-shell resonances.

Because of the rapid Coster-Kronig decay of the sulfur $2 \mathrm{~s}$ core hole, all features observed in the $L_{1}$ region are broad, as compared to the sharp structure near the sulfur $L_{2,3}$ edges. The calculated natural linewidth for atomic sulfur is $1.49 \mathrm{cV}$ for a $2 \mathrm{~s}$ core hole. compared to $54 \mathrm{meV}$ for a $2 p$ core hole [22]. Sulfur $2 \mathrm{~s}$ peaks in the XPS spectra of several sulfur-containing compounds were observed to be much wider than the corresponding sulfur $2 p$ peaks [48]. If the vibrational structure proposed here for the $(2 s)^{-1}$ valence-shell resonances were confirmed, it would indicate a much nartower linewidth than predicted by the theoretical and XPS results.

\section{VI.I. Comparison to 'Theoretical Results}

Comparisons to theoretical work have been mentioned, as appropriate, in the preceding sections. Only a few points need further comment here. There have been several theoretical studies of the $\mathrm{L}_{2,3}$ XANES spectrum of $\mathrm{H}_{2} \mathrm{~S}[4,15,34,43]$. While none of these theoretical results accurately predict the fine structure observed in the present measurements, some of these results were used as a guide in the assignments given here. 
Specifically, Schwarz $[4]$ and Cacelli, et. al. $[15]$ both predict transitions to 4 p-derived othitals with term values of $=2.4 \mathrm{eV}$, as also predicted hy the quantum defect derived here for the higher np otbitals. These results suggest a strong $4 p$ contribution to the mixed states. Cacelli, et. al., predict relatively intense transitions to orbitals having mainly $3 \mathrm{~d}$ and ss contributions with term values of $=1.7-2.0 \mathrm{eV}$, again in agreement with the predictions of quantum defects derived here for the higher Rydberg orbitals. Recent calculations by Liu, et. al. (34) suggest that transitions to the higher np ortitals are more intense than to the higher ns orbitals. This supports the assignment deduced here on the hasis of consistent quantum defects.

In one calculation, the molecular-field splitting of the sulfur $2 \mathrm{p} 3 / 2$ level was predicted to be $44 \mathrm{meV}$ for core-ionized $\mathrm{H}_{2} \mathrm{~S}^{+}[49]$. Another calculation estimated that splitting to be $80 \mathrm{meV}[50]$. Because the Rydherg electron should have only a small influence at the atomic core. these values can be compared to the $115 \mathrm{meV}$ splitting derived here for the Rydberg states. Agrecment with the calculations is fair, considering the $=170$ eV total energy of the excited state.

\section{VI.J. Conclusions}

In the present work, high-resolution low-noise XANES spectra of $\mathrm{H}_{2} \mathrm{~S}$ and $\mathrm{D}_{2} \mathrm{~S}$ below the sulfur $L_{2,3}$ edges were measured, providing significant improvements over previous measurements. Analysis of these spectra clearly indicated that the sulfur $2 p_{3} / 2$ core level is split into two levels by the anisotropic molecular field. That splitting is measured to be $115 \mathrm{meV}$ for the Rydherg excitations, in good agreement with the splitting proposed for the core-ionized molecule hased on high-resolution XPS and AES spectra [7]. A sophisticated least-squares analysis was applied to the data. allowing the deconvolution of core-level and excited-ortital splittings. This approach will also be useful for the analysis of complex high-resolution XANES spectra of other molecules. The comparison 
of the $\mathrm{H}_{2} \mathrm{~S}$ spectrum to the corresponding $\mathrm{D}_{2} \mathrm{~S}$ spectrum was essential in this analysis for the identification of vibrational excitations. Quantum defects were derived for the higher Rydberg ortitals which were similar to the best avallable quantum defects from the corresponding spectra of the isoclectronic systems $\mathrm{SiH}_{4}, \mathrm{PH}_{3}, \mathrm{HCl}$, and $\mathrm{Ar}$. Fine structure was observed for the transitions to the dissociative valence-shell states. The regular spacing and isotopic dependence of this structure clearly identifies it as a vibrational progression.

Besides their relevance to core-excitations in general, the present results for $\mathrm{H}_{2} \mathrm{~S}$ provide information about the core-equivalent molecule $\mathrm{H}_{2} \mathrm{Cl}$ [4]. Specifically, the term values derived here for the mixed and Rydberg orbitals of core-excited $\mathrm{H}_{2} \mathrm{~S}$ should be fairly close to the term values of the corresponding orbitals of valence-excited $\mathrm{H}_{2} \mathrm{Cl}$. These results provide a useful comparison for theoretical results, in the absence of any direct information on the $\mathrm{H}_{2} \mathrm{Cl}$ radical, which presumably dissociates in its ground clectronic state. The first few excited electronic states of this radical are of particular interest as they may influence the dynamics of the $\mathrm{HCl}+\mathrm{H}=\mathrm{H}+\mathrm{ClH}$ exchange reaction. 


\section{REFERENCES:}

1. K. Sieghahn, C. Nordling, G. Johansson, J. Hedman, P. F. Hedén, K. Hamrin, U. Gellus, and T. Bergmark, ESCA Applied to Free Molecules (North-Holland, Amsterdam. 1969), pp. 104-136; D. A. Shirley. Chem. Phys. Lett. 16, 220 (1972); U. Gelius. Phys. Script. 9, 133 (1974); L. Asplund, P. Kelfve, H. Siegbahn, O. Goscinski, H. FellnerFeldegg, K. Hamrin, B. Blomster, and K. Sieghahn, Chem. Phys. Lett. 40, 3.53 (1976); N. D. Lang and A. R. Williams. Phys. Rev. B 16. 2408 (1977).

2. D. Thomas. M. Coville, R. Thissen, and P. Morin, Synchrotron Radiat. News 5, No. 3. 9 (1992).

3. W. Hayes and F. C. Brown, Phys. Rev. A 6. 21 (1972).

4. W. H. E. Schwarz, Chem. Phys. 11, 217 (1975).

5. M. B. Kobin. Chem. Phys. Lell. 31, 140 (1975).

6. C. Sandorfy. Top. Curr. Chem. 86, 91 (1979).

7. S. Svensson. A. Naves de Brito, M. P. Keane, N. Correia, and L. Karlsson, Phys. Rev. A 43, 6441 (1991).

8. K. Sieghahn, C. Nordling, G. Johansson, J. Hedman, P. F. Heden, K. Hamrin, U. Gelius, and T. Bergmark. ESCA Applied to Free Molecules (North.Holland. Ainsterdam, 1969), pp.84-87.

9. R. G. Cavell and K. H. Tan, Chem. Phys. Lell. 197, 161 (1992).

10. F. J. Comes, R. Haensel, U. Nielson, and W. H. E. Schwarz. J. Chem. Phys. 58. $516(1973)$;

II. R. P. Gupta, J. S. Tse, and G. M. Bancroft, Philos. Trans. Roy. Soc. 293, 535 (1980); G. M. Bancroft and J. S. Tse, Comments Inorg. Chem. 5. 89 (1986); J. N. Cutler, G. M. Buncroft, and K. H. Tan. J. Chem. Phys. 97, 79.32 (1992).

12. K. Ninomiya, E. Ishiguro, S. Iwata, A. Mikuni, and T. Sasaki, J. Phys. B 14, 1777 (1981).

13. E. Ishiguro, S. Iwata, A. Mikuni, Y, Suzuki, H. Kanamori, and T. Sasaki, J, Phys. B 20, 4725 (1897).

14. N. A. Shklyaeva, L. N. Mazalov, and V. V. Murakhtanov, J. Struct. Chem. 20, 621 (1979); K. H. Sze, C. E. Brion, X. M. Tong, and J. M. Li, Chem. Phys, 115. 433 (1987).

15. I. Cacelli, V. Carravetta, and R. Moccia, Chem. Phys. 120. 51 (1988).

16. P. Morin, M. Lavollee, and M. Simon, to be published.

17. H. Aksela. S. Aksela, A. Naves de Brito, G. M. Bancroft, and K. H. Tan, Phys. Rev. A 45, 7948 (1992). 
18. A. N. de Brito and H. Agren, Phys. Rev. A 45, 7953 (1992).

19. R. E. LaVilla, J. Chem. Phys. 62, 2209 (1975); S. Bodeur and J. M. Esteva, Chem. Phys. 100, 415 (1985); R. Mayer, D. W. Lindle, S. H. Southworth, and P. L. Cowan, Phys. Rev. A 43, 235 (1991).

20. C. T. Chen, Y. Ma, and F. Sette, Phys. Rev. A 40, 6737 (1989).

21. For example, see Chapter IV of this dissertation.

22. M. O. Krause and J. H. Oliver, J. Phys. Chem. Ref. Data 8, 329 (1979).

23. M. Weissbluth, Atoms and Molecules, (Academic Press, San Francisco, 1978) pp. $385-402$.

24. R. D. Cowan, The Theory of Atomic Structure and Spectra (University of California Press, Berkeley, 1981) pp. 134-135.

25. U. Nielson, R. Haensel, and W. H. E. Schwarz, J. Chem. Phys. 61, 3581 (1974);

Y. Onodera and Y. Toyozawa, J. Phys. Soc. Japan 22, 833 (1967).

26. R. L. Cook, F. C. DeLucia, and P. Helminger, J. Mol. Struct. 28, 237 (1975).

27. For example, see Chapters III and V of this dissertation.

28. Y. Ma, C. T. Chen, G. Meigs, K. Randall, and F. Sette, Phys. Rev. A 44, 1848 (1991); F. X. Gadea, H. Köppel, J. Schirmer, L. S. Cederbaum. K. J. Randall, A. M. Bradshaw, Y. Ma, F. Sette, C. T. Chen, Phys. Rev. Lett. 66, 883 (1991); J. Schirmer, A. B. Trofimov, K. J. Randall, J. Feldhaus, A. M. Bradshaw, Y. Ma, C. T. Chen, and F. Sette, Phys. Rev. A 47, 1136 (1993).

29. M. Domke, T. Mandel, A. Puschmann, C. Xue, D. A. Shirley, G. Kaindl, H. Petersen, and P. Kuske, Rev. Sci. Instrum. 63, 80 (1992).

30. G. C. King, M. Tronc, F. H. Read, and R. C. Bradford, J. Phys. B 10, 2479 (1977).

31. G. Herzberg, Molecular Spectra and Molecular Structure (Van Nostrand Reinhold, New York, 1966) Vol. III, pp.17-18.

32. U. Fano and J. W. Cooper, Rev. Mod. Phys. 40, 441 (1968); D. A. Outka and J. Stöhr, J. Chem. Phys. 88, 3539 (1988).

33. L. Halonen and T. Carrington, Jr., J. Chem. Phys. 88, 4171 (1988).

34. Z. F. Liu, G. M. Bancroft and J. S. Tse, to be published.

35. Derived from the analysis presented in Chapter V (see Sections V.D.1 and V.E.1, Tables V-1,2, and Figs. V-2,4).

36. H. Friedrich, B. Sonntag, P. Rabe, W. Butscher, and W. H. E. Schwarz, Chem. Phys. Lett. 64, 360 (1979).

37. D. G. J. Sutherland, G. M. Bancroft, J. D. Bozek, and K. H. Tan, Chem. Phys. 
Lett. 199, 341 (1992).

38. R. N. S. Sodhi and C. E. Brion, J. Electron Spectrosc. Relat. Phenom. 37, 97 (1985).

39. Z. Liu, J. N. Cutler, G. M. Bancroft, K. H. Tan, R. G. Cavell, and J. S. Tse, Chem. Phys. Lett. 172, 421 (1990).

40. D. A. Shaw, D. Cvejanovic, G. C. King, and F. H. Read, J. Phys. B 14, 1777 (1981).

41. Y. Hahn, Phys. Rev. A 13, 1326 (1976).

42. L. S. Cederbaum, J. Chem. Phys. 78, 5714 (1983).

43. H. Nakamatsu, T. Mukoyama, and H. Adachi, J. Chem. Phys. 95, 3167 (1991).

44. L. C. Lee, X. Wang, and M. Suto, J. Chem. Phys. 86, 4353 (1987).

45. X. Xie, L. Schnieder, H. Wallmeier, K. H. Welge, and M. N. R. Ashfold, J. Chem. Phys. 92, 1608 (1990) and references therein.

46. K. Weide, V. Staemmler, and R. Schinke, J. Chem. Phys. 93, 861 (1990); B.

Heumann, R. Duren, and R. Schinke, Chem. Phys. Lett. 180, 583 (1991).

47. M. Braunstein and R. T. Pack, J. Chem. Phys. 96, 891 (1992).

48. U. Gelius, J. Electron Spectrosc. Relat. Phenom. 5, 985 (1974).

49. D. Thomas, private communication.

50. A. Cesar and H. Agren, unpublished, cited in Ref. [7]. 
Table VI-1: $\mathrm{H}_{2} \mathrm{~S}$ and $\mathrm{D}_{2} \mathrm{~S}(2 \mathrm{p})^{-1}$ Rydberg resonances: Assignments and fit results. The energies tabulated apply to states with the $\left(2 p_{1 / 2}\right)^{-1}$ core vacancy. Energies for corresponding states with $\left(2 \mathrm{p}_{3 / 2}-4 \mathrm{e}_{1 / 2}\right)^{-1}$ and $\left(2 \mathrm{p}_{3 / 2}-5 \mathrm{e}_{1 / 2}\right)^{-1}$ core vacancies can be obtained by subtraction of term values from the tabulated energies of the $\mathrm{L}_{3}-4 \mathrm{e}_{1 / 2}$ and $\mathrm{L}_{3}-5 \mathrm{e}_{1 / 2}$ edges. The Rydberg orbital assignments shown apply to all three possible core vacancies. The intensity ratio $\mathrm{X}$ is a dimensionless fit parameter defined in the text.

\begin{tabular}{|c|c|c|c|c|c|c|c|}
\hline & $\mathrm{H}_{2} \mathrm{~S}$ & & Assignment & & & $\mathrm{D}_{2} \mathrm{~S}$ & \\
\hline $\begin{array}{c}\text { Energy } \\
(\mathrm{eV})\end{array}$ & $\begin{array}{l}\text { Term } \\
\text { Value } \\
(\mathrm{eV})\end{array}$ & $\begin{array}{l}\text { Inten- } \\
\text { sity }\end{array}$ & & & $\begin{array}{c}\text { Energy } \\
(\mathrm{eV})\end{array}$ & $\begin{array}{l}\text { Term } \\
\text { Value } \\
(\mathrm{eV})\end{array}$ & $\begin{array}{l}\text { Inten- } \\
\text { sity }\end{array}$ \\
\hline $\begin{array}{l}169.535 \\
169.577 \\
169.688 \\
169.833 \\
169.935 \\
170.134 \\
170.303 \\
170.315 \\
170.353 \\
170.418 \\
170.466 \\
170.534 \\
170.604 \\
170.670 \\
170.807 \\
170.851 \\
170.943 \\
171.092 \\
171.142 \\
171.228 \\
171.259 \\
171.333 \\
171.564\end{array}$ & $\begin{array}{l}2.029 \\
1.987 \\
1.876 \\
1.731 \\
1.629 \\
1.430 \\
\\
1.249 \\
1.211 \\
\\
1.098 \\
1.030 \\
0.960 \\
0.894 \\
0.757 \\
0.713 \\
0.621 \\
0.472 \\
0.422 \\
0.336 \\
0.305 \\
0.231 \\
0\end{array}$ & $\begin{array}{r}442 \\
589 \\
1010 \\
660 \\
399 \\
81 \\
298 \\
192 \\
\\
90 \\
1175 \\
243 \\
240 \\
118 \\
115 \\
1000 \\
46 \\
546 \\
10 \\
281 \\
129 \\
\ldots\end{array}$ & $\begin{array}{c}\text { 3d-derived } \\
\text { 3d-derived } \\
\text { 3d-derived } \\
\text { 3d-derived } \\
5 s \text {-derived? } \\
? \\
\text { ? } \\
\mathrm{L}_{3}-5 \mathrm{e}_{1 / 2} \text { edge } \\
5 \mathrm{p} \text {-derived } \\
5 \mathrm{p} \text {-derived } \\
\mathrm{L}_{3}-4 \mathrm{e}_{1 / 2} \text { edge } \\
\text { vibration } \\
4 \mathrm{~d}-\text {-derived } \\
4 \mathrm{~d}-\text { derived } \\
6 \mathrm{~s} ? \\
? \\
6 \mathrm{p} \\
5 \mathrm{~d} \\
7 \mathrm{p} \\
6 \mathrm{~d} \\
8 \mathrm{p} \\
7 \mathrm{~d} \\
8 \mathrm{~d} \\
\mathrm{~L}_{2} \text { edge }\end{array}$ & $\begin{array}{l}0.20 \\
1.07 \\
1.72 \\
0.78 \\
0.70 \\
2.50 \\
\\
1.14 \\
0.79 \\
\\
2.50 \\
1.01 \\
0.98 \\
0.20 \\
0.76 \\
0.88 \\
1.04 \\
1.00 \\
1.00 \\
1.00 \\
1.00 \\
1.00 \\
\ldots\end{array}$ & $\begin{array}{l}169.527 \\
169.557 \\
169.680 \\
169.822 \\
169.933 \\
170.143 \\
170.303 \\
170.309 \\
170.351 \\
170.418 \\
170.423 \\
170.532 \\
170.601 \\
170.667 \\
170.791 \\
170.851 \\
171.943 \\
171.092 \\
171.142 \\
171.228 \\
171.259 \\
171.333 \\
171.564\end{array}$ & $\begin{array}{l}2.037 \\
2.007 \\
1.884 \\
1.742 \\
1.631 \\
1.421 \\
\\
1.255 \\
1.213 \\
\\
1.141 \\
1.032 \\
0.963 \\
0.897 \\
0.773 \\
0.713 \\
0.621 \\
0.472 \\
0.422 \\
0.336 \\
0.305 \\
0.231 \\
0\end{array}$ & $\begin{array}{r}518 \\
626 \\
930 \\
885 \\
391 \\
116 \\
\\
267 \\
210 \\
\\
94 \\
1180 \\
249 \\
243 \\
118 \\
114 \\
1000 \\
41 \\
549 \\
11 \\
283 \\
127 \\
\ldots\end{array}$ \\
\hline
\end{tabular}


Table VI-2: $\mathrm{H}_{2} \mathrm{~S}$ and $\mathrm{D}_{2} \mathrm{~S}(2 \mathrm{p})^{-1}$ mixed resonances: Fit results and vibrational assignments. The energies tabulated apply to states with the $\left(2 \mathrm{p}_{1 / 2}\right)^{-1}$ core vacancy. Energies for corresponding states with $\left(2 \mathrm{p}_{3 / 2}-4 \mathrm{e}_{1 / 2}\right)^{-1}$ and $\left(2 \mathrm{p}_{3 / 2}-5 \mathrm{e}_{1 / 2}\right)^{-1}$ core vacancies can be obtained by subtraction of term values from $170.471 \mathrm{eV}$ and $170.432 \mathrm{eV}$, respectively. The intensity ratio $\mathrm{X}$ is a dimensionless fit parameter defined in the text. The assignments shown apply to all three possible core vacancies. Tentative assignments of vibrational progressions are identified by $\mathrm{A} 1, \mathrm{~A} 2$, etc. The energy spacing between succesive states of a progression, e.g. $\mathrm{E}_{\mathrm{A} 2}-\mathrm{E}_{\mathrm{A} 1}$, is also listed.

\begin{tabular}{|c|c|c|c|c|c|}
\hline $\begin{array}{l}\text { Energy } \\
(\mathrm{eV})\end{array}$ & $\begin{array}{c}\text { Term Value } \\
(\mathrm{eV})\end{array}$ & Intensity & $\begin{array}{c}\text { Intensity } \\
\text { Ratio } \\
\text { (X) }\end{array}$ & $\begin{array}{l}\text { Vibrational } \\
\text { Assignment }\end{array}$ & $\begin{array}{c}\text { Vibrational } \\
\text { Spacing } \\
(\mathrm{meV})\end{array}$ \\
\hline \multicolumn{6}{|l|}{$\mathrm{H}_{2} \mathrm{~S}$} \\
\hline $\begin{array}{l}168.371 \\
168.407 \\
168.564 \\
168.716 \\
168.806 \\
168.872 \\
168.923 \\
169.040 \\
169.190 \\
169.340 \\
169.443\end{array}$ & $\begin{array}{l}3.193 \\
3.157 \\
3.000 \\
2.848 \\
2.758 \\
2.692 \\
2.641 \\
2.524 \\
2.374 \\
2.224 \\
2.121\end{array}$ & $\begin{array}{r}91 \\
33 \\
246 \\
505 \\
629 \\
524 \\
204 \\
457 \\
260 \\
167 \\
122\end{array}$ & $\begin{array}{l}0.20 \\
2.04 \\
2.50 \\
0.96 \\
0.88 \\
1.04 \\
1.38 \\
1.18 \\
0.52 \\
0.20 \\
1.23\end{array}$ & $\begin{array}{l}\text { A1 } \\
\text { A2 } \\
\text { A3 } \\
\text { A4 } \\
\\
\text { A5 } \\
\text { A6 } \\
\text { A7 }\end{array}$ & $\begin{array}{l}157 \\
152 \\
\\
156 \\
\\
164 \\
150 \\
150\end{array}$ \\
\hline \multicolumn{6}{|l|}{$\mathrm{D}_{2} \mathrm{~S}$} \\
\hline $\begin{array}{l}168.377 \\
168.514 \\
168.632 \\
168.680 \\
168.711 \\
168.785 \\
168.850 \\
168.990 \\
169.166 \\
169.341\end{array}$ & $\begin{array}{l}3.187 \\
3.050 \\
2.932 \\
2.884 \\
2.853 \\
2.779 \\
2.714 \\
2.574 \\
2.398 \\
2.223\end{array}$ & $\begin{array}{r}364 \\
157 \\
150 \\
47 \\
105 \\
721 \\
747 \\
756 \\
318 \\
176\end{array}$ & $\begin{array}{l}0.20 \\
2.49 \\
2.50 \\
0.32 \\
1.84 \\
0.88 \\
1.10 \\
1.45 \\
0.20 \\
0.20\end{array}$ & $\begin{array}{l}\mathrm{A} 1 \\
\mathrm{~A} 2 \\
\mathrm{~A} 3\end{array}$ & $\begin{array}{l}139 \\
140\end{array}$ \\
\hline
\end{tabular}


Table VI-3 : Quantum defects for Rydberg states in the $\mathrm{L}_{2,3}$ XANES spectra of Ar and the second-row hydrides.

\begin{tabular}{cccccc}
\hline & $\mathrm{SiH}_{4}{ }^{\mathrm{a}}$ & $\mathrm{PH}_{3}{ }^{\mathrm{b}}$ & $\mathrm{H}_{2} \mathrm{Sc}^{\mathrm{c}}$ & $\mathrm{HCl}$ & $\mathrm{Ar}$ \\
\hline$\delta_{\mathrm{s}}$ & 2.8 & 2.3 & 2.1 & 2.12 & $2.20 \mathrm{e}$ \\
$\delta_{\mathrm{p}}$ & 1.73 & 1.8 & 1.63 & 1.7 & $1.74 \mathrm{f}$ \\
$\delta_{\mathrm{d}}$ & 0.2 and $0.4 \mathrm{~g}$ & 0.44 & 0.32 & 0.3 & $0.20^{\mathrm{e}}$ \\
\hline
\end{tabular}

a Ref. [37].

b Derived from term values given in Ref. [13].

c This work.

d Ref. [40].

e Derived from the spectrum in Ref. [29].

f Ref. [30].

g In $\mathrm{SiH}_{4}$ the d-series splits into d-t $\mathrm{t}_{2}$ and d-e. 
Table VI-4: Results of least-squares analysis of $(2 p)^{-1}$ valence-shell region: Approximate fits of overall lineshapes.

\begin{tabular}{|c|c|c|c|}
\hline & $\begin{array}{l}\text { Spin-Orbit } \\
\text { Splitting } \\
\text { (eV) }\end{array}$ & $\begin{array}{c}\text { Intensity Ratio } \\
\left(2 \mathrm{p}_{3 / 2}\right)^{-1} 6 \mathrm{a}_{1}:\left(2 \mathrm{p}_{1 / 2}\right)^{-1} 6 \mathrm{a}_{1}\end{array}$ & $\begin{array}{c}\text { Intensity Ratio } \\
\left(2 \mathrm{p}_{3 / 2}\right)^{-1} 3 \mathrm{~b}_{2}:\left(2 \mathrm{p}_{1 / 2}\right)^{-1} 3 \mathrm{~b}_{2}\end{array}$ \\
\hline $\begin{array}{l}\mathrm{H}_{2} \mathrm{~S} \\
\Gamma_{2} \mathrm{~S}\end{array}$ & $\begin{array}{l}1.07 \\
1.09\end{array}$ & $\begin{array}{l}1.8 \\
2.1\end{array}$ & $\begin{array}{l}1.2 \\
1.1\end{array}$ \\
\hline
\end{tabular}

Table VI-5: Results of least-squares analysis of $(2 p)^{-1}$ valence-shell region: Exact fit of fine structure. Energies of peaks, with uncertainties, and energy spacings between successive peaks are tabulated for $\mathrm{H}_{2} \mathrm{~S}$ and $\mathrm{D}_{2} \mathrm{~S}$.

\begin{tabular}{|c|c|c|c|}
\hline$\underset{(\mathrm{eV})}{\text { Energy }}$ & $\begin{array}{l}\mathrm{H}_{2} \mathrm{~S} \\
\text { Spacing (meV) }\end{array}$ & $\begin{array}{c}\text { Energy } \\
(\mathrm{eV})\end{array}$ & $\begin{array}{l}\mathrm{D}_{2} \mathrm{~S} \\
\text { Spacing (meV) }\end{array}$ \\
\hline $\begin{array}{l}165.037(9) \\
165.204(6) \\
165.371(7) \\
165.536(9) \\
165.706(9)\end{array}$ & $\begin{array}{l}167 \\
167 \\
165 \\
170\end{array}$ & $\begin{array}{l}164.998(9) \\
165.146(8) \\
165.289(7) \\
165.428(7) \\
165.577(9)\end{array}$ & $\begin{array}{l}148 \\
143 \\
139 \\
149\end{array}$ \\
\hline
\end{tabular}




\section{FIGURE CAPTIONS:}

Figure VI-1. Overview of the photoionization spectrum of $\mathrm{H}_{2} \mathrm{~S}$ near the sulfur $\mathrm{L}$ edges. The positions of the ionization thresholds determined from analysis of the spectra are also shown. The small molecular-field splitting of the $\mathrm{L}_{3}$ edge is not visible with this energy scale. The inset shows a higher-resolution spectrum in the $178.56-175.56 \mathrm{eV}$ range, with the energy scale expanded by a factor of five. The features in this range are attribuled to multi-electron excitations.

Figure VI-2. High-resolution photoionization spectra of $\mathrm{H}_{2} \mathrm{~S}$ and $\mathrm{D}_{2} \mathrm{~S}$ in the mixed and Rydberg region of the sulfur $\mathrm{L}_{2,3}$ edges. Rydberg assignments applying to both spectra are shown in the bar diagrams, identifying s-,p-, and d-series converging on each of the three ionization thresholds. Note that the $s$ assignments are less certain than the others. For the mixed states, different assignments are shown for the two spectra, because of the large isotopic differences for these states. The isotopic shift of the $\left(2 \mathrm{p}_{1 / 2}\right)^{-1} 5 \mathrm{p}$ vibrational sideband, as determined from the fit, is indicated by the vertical line. The data points are plotted as circles. The results of a least-squares analysis are plotted as solid lines. The contributing subspectra plotted underneath, with dashed lines showing features attributed to $2 p_{1 / 2}$ core-hole states, solid lines showing features attributed to $2 p_{3 / 2}-4 e_{1 / 2}$ core-hole states, and dotted lines showing features attributed to $2 \mathrm{p}_{1 / 3}-5 \mathrm{e}_{1 / 2}$ core-hole states. See Sec. VI.E.3 a for further details.

Figure VI-3. High-resolution photoionizationtion spectra of $\mathrm{H}_{2} \mathrm{~S}$ and $\mathrm{D}_{2} \mathrm{~S}$ in the region of valence shell excitations below the sulfur $L_{2,3}$ edges. The vertical lines indicate isotopic shifts in some features of the peak. Portions of the data are also shown with the vertical scale expanded, to display the weakly resolved fine structure. The results of a leastsquares analysis are plotted as solid lines with the expanded data. For $\mathrm{D}_{2} \mathrm{~S}$, the fit line is 
not visible, due the large point density and the high quality of the fit.

Figure VI-4. High-resolution photoionization spectra of $\mathrm{H}_{2} \mathrm{~S}$ and $\mathrm{D}_{2} \mathrm{~S}$ near the sulfur $\mathrm{L}_{1}$ edge. A linear background was subtracted from the data for plotting purposes. Note the weak feature at $233.4 \mathrm{eV}$. The region $227-229 \mathrm{eV}$, which shows slight indications of fine structure, is shown in the insets with both the energy and intensity scales expanded by a factor of three.

Figure VI-5. Alternative fits to the data of Fig. VI-2, assuming there is no molecular-field splitting of the sulfur $2 \mathrm{p}_{3 / 2}$ core level. In the subspectra, the dashed lines show features attributed to $2 p_{1 / 2}$ core-hole states and the solid lines show features attributed to $2 \mathrm{p}_{3} / 2$ corehole states. Note the poorer agreement with data for these fits, as compared to the fits in Fig. VI-2, particularly in the $169.0-169.4 \mathrm{eV}$ range. 


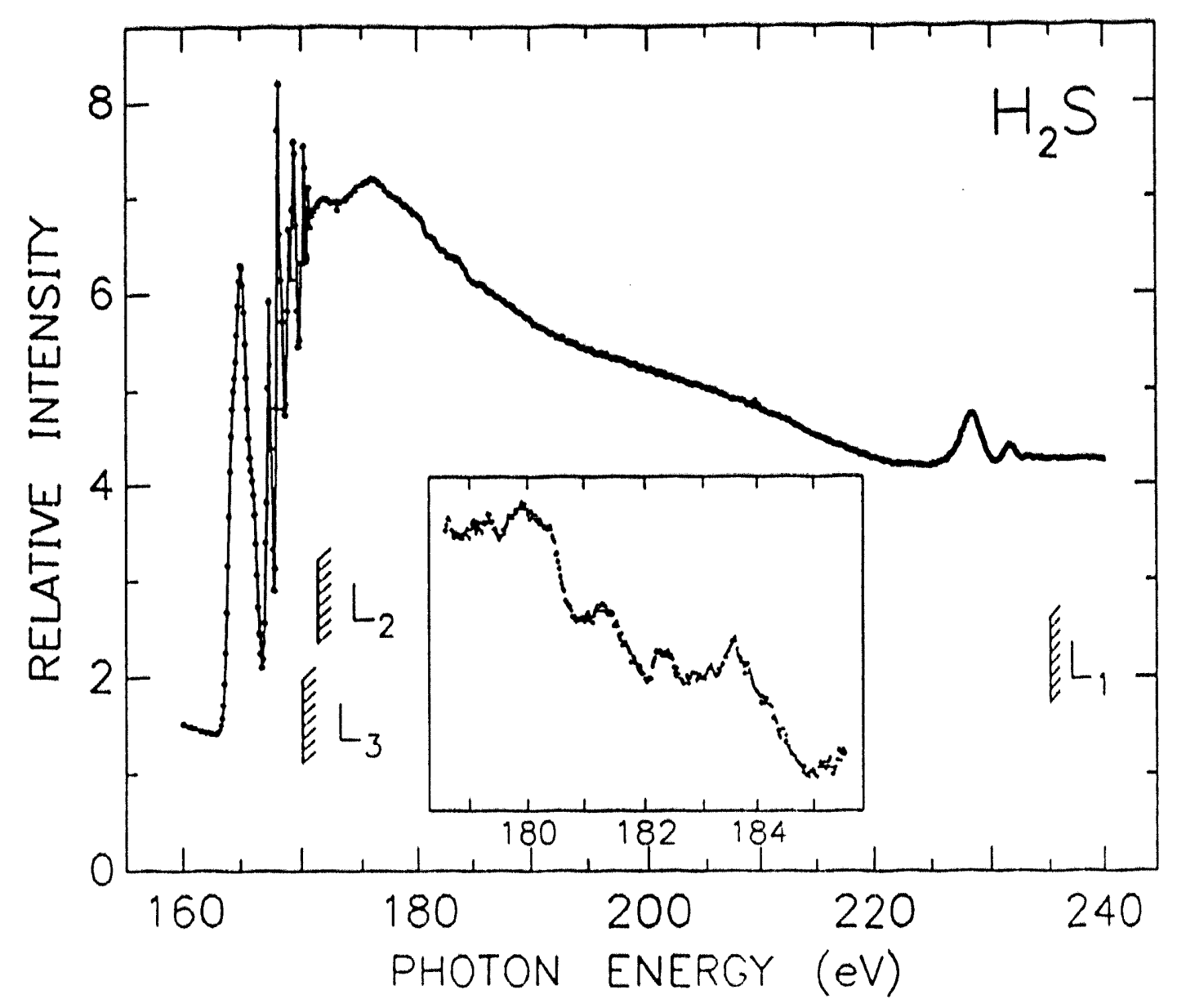

Eigure VI-1 


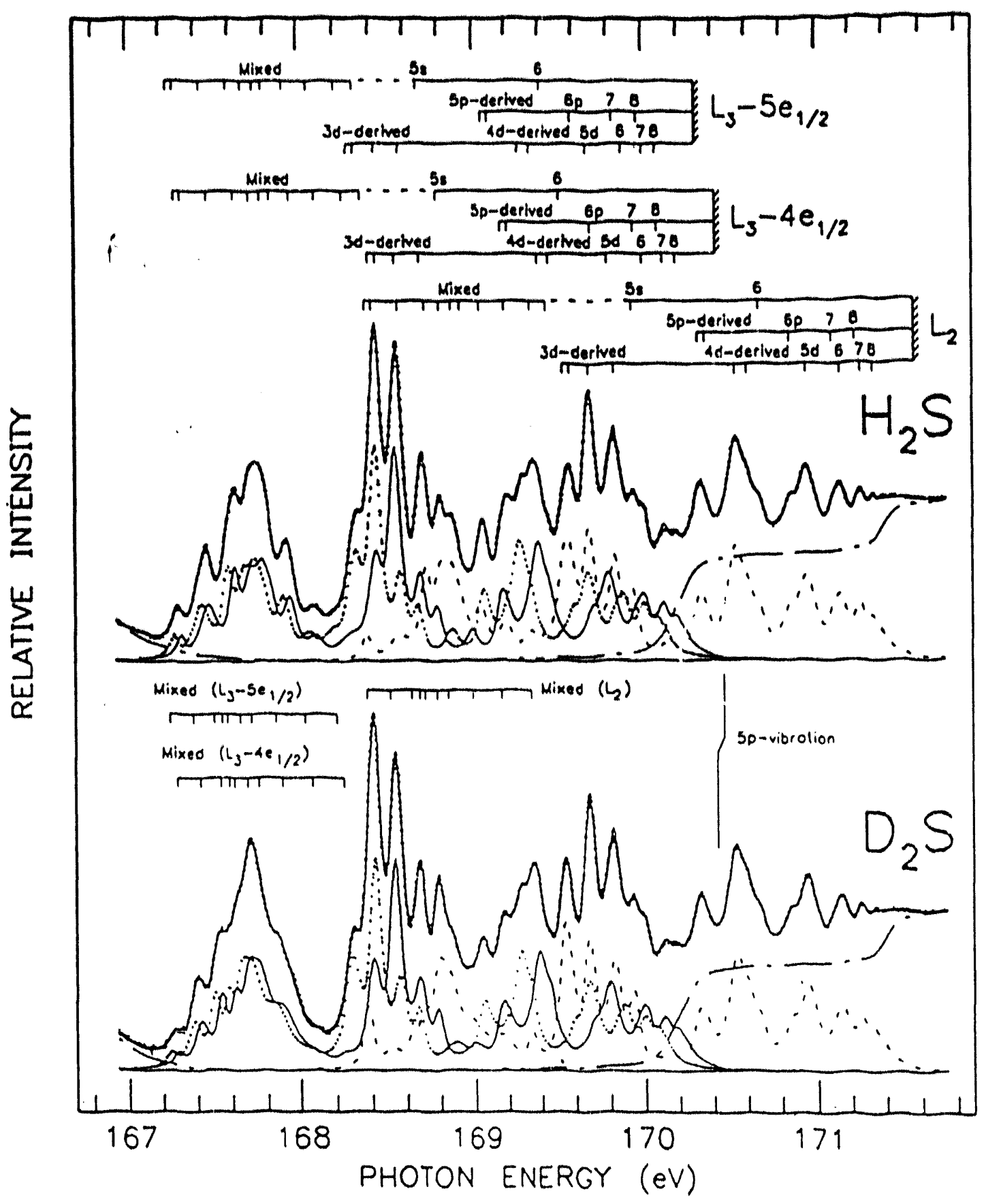

Figure VI-2 


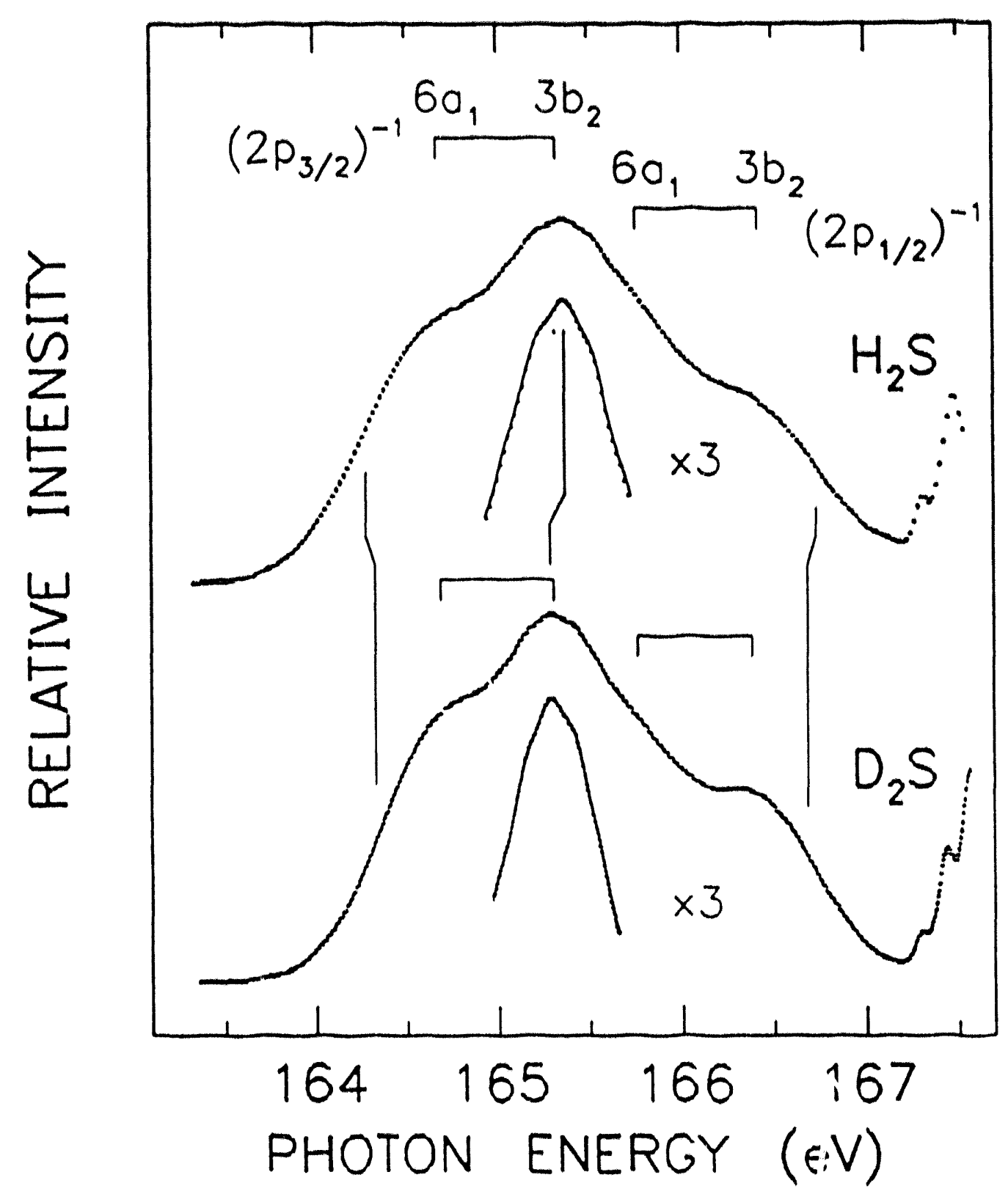

Figure VI-3 


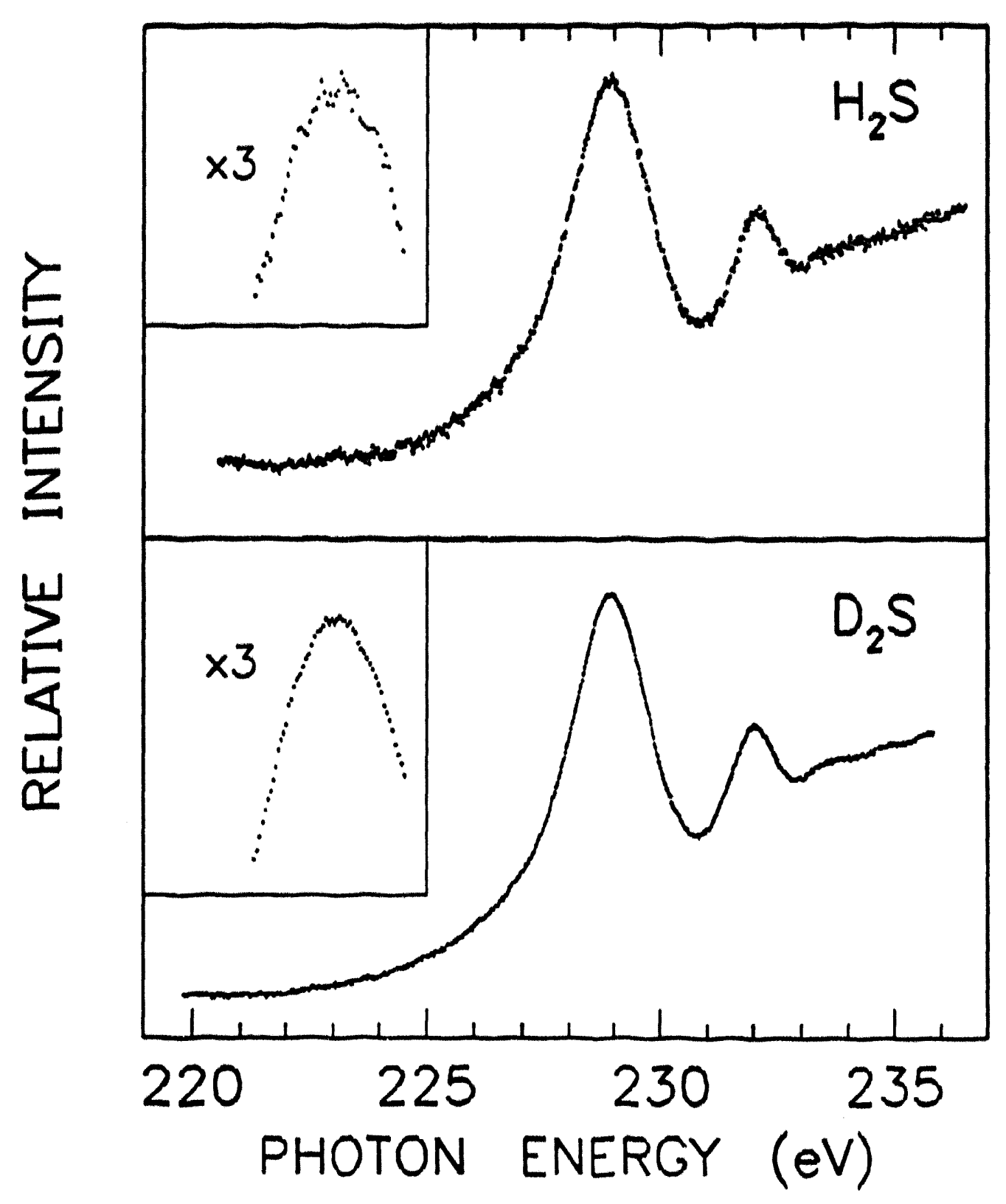

Figure VI-4 


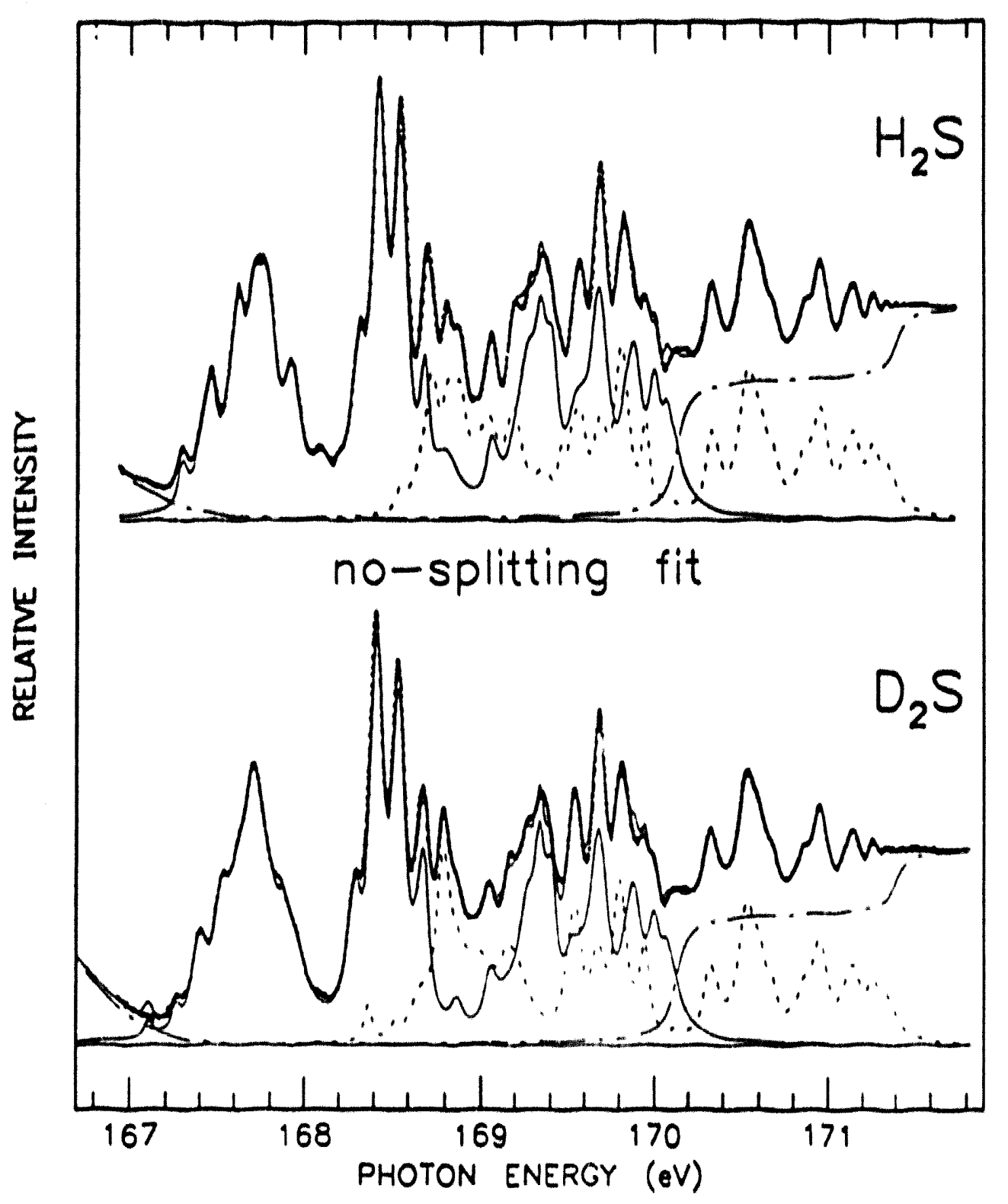

Figure VI-5 


\section{CHAPTER VII: FUTURE DIRECTIONS}

\section{VII.A. Improvements in the present spectra}

The present results are limited less by instrumental factors than by natural linewidths of the excited states. Nonetheless, improvements in flux and resolution from soft $x$-ray monochromators should reveal additional fine structure in nearly all the spectra presented here. Besides the general possibility of enhancing the present understanding of these spectra, there are a few specific features which warrant re-examination with improved monochromators.

The oxygen K-edge spectra of $\mathrm{H}_{2} \mathrm{CO}$ and $\mathrm{D}_{2} \mathrm{CO}$ presented in Section IV.D. did not show any fine structure. Spectra with better instrumental resolution will probably be able to resolve vibrational features in the $(\mathrm{O} 1 \mathrm{~s})^{-1} \pi^{*}$ and Rydberg resonances. This would provide a much better understanding of the structure and bonding in these core-excited states. The isotopic shifts of the $(\mathrm{O} 1 \mathrm{~s})^{-1} 3 \mathrm{~s}$-a 1 resonance are particularly worthy of further study, because of the analogous effects seen for the better-resolved (C 1s)-1 3s-a 1 resonance (see Section IV.E.2).

In Section V.D.2.a, the possibility of fine structure in the $(S 2 p)^{-1} a_{1 g}$ resonances of $\mathrm{SF}_{6}$ was discussed. The present data is inconclusive, but suggests that some shoulders may be resolvable in these peaks. Success in resolving vibrational structure would be of interest, because the Franck-Condon analysis gave a $v_{1}$ vibrational spacing larger than that of the ground state. Such an increase in vibrational energy is difficult to explain for a transition to an orbital which should be anti-bonding. Better-resolved spectra might allow direct measurement of the vibrational spacing in this excited state.

The (S 2s) ${ }^{-1} 3 b_{2}, 6 a_{1}$ valence-shell resonances of $\mathrm{H}_{2} \mathrm{~S}$ and $\mathrm{D}_{2} \mathrm{~S}$ were presented in Section VI.H. Here there were also faint indications of vibrational structure, which could not be confirmed. If such structure can be observed, it will indicate a natural linewidth for 


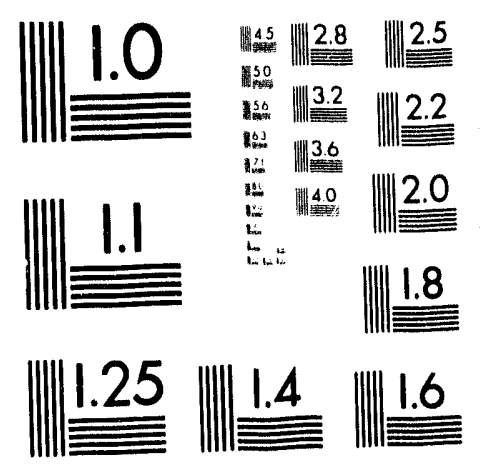



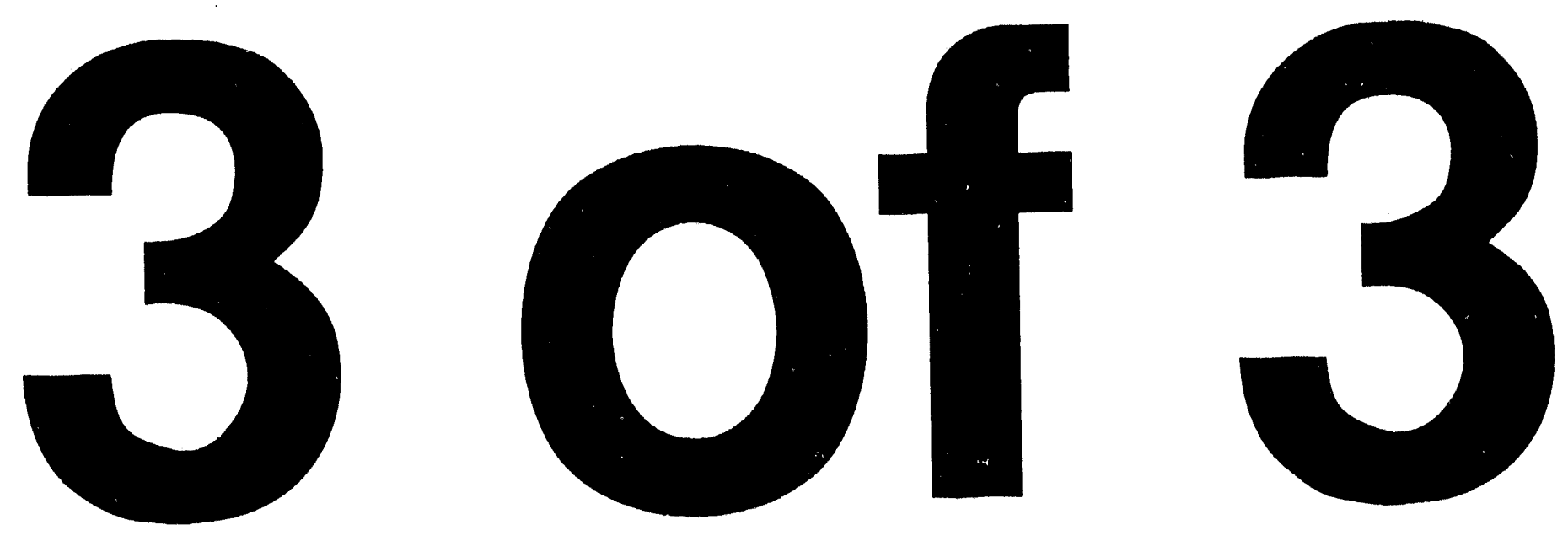
the excited state which is much less than the $1.49 \mathrm{eV}$ predicted for the sulfur $2 \mathrm{~s}$ core hole [1]. In the (S 2p)-1 mixed and Rydberg resonances, the analysis indicated that there are many overlapping peaks (see Section VI.E.4). A better-resolved spectrum will distinguish more features, allowing a more detailed analysis. In particular, the vibrational structure of the (S $2 p)^{-1}$ mixed resonances was difficult to fully interpret with the present results (see Section IV.E.6).

\section{VII.B. High-resolution spectra of other molecules}

The XANES spectra of a number of molecules have been re-examined with high resolution using the new generation of soft $x$-ray monochromators, as discussed in Section I.B. Nonetheless, there are interesting systems which have not been studied with modern high-resolution. Most of the recent work has focussed on the carbon, nitrogen, and oxygen $\mathrm{K}$ edges. There are still possiblities for improved spectra at lower-energy core levels. In particular, the present results for $\mathrm{H}_{2} \mathrm{~S}$ suggest further experiments at $\mathrm{L}_{2,3}$ edges of the isoelectronic hydrides $\mathrm{SiH}_{4}, \mathrm{PH}_{3}$, and $\mathrm{HCl}$. The $\mathrm{L}_{2,3} \mathrm{XANES}$ or EELS spectra of these molecules have all been measured with fairly high resolution (see Section VI.E.5.b for references). However, better combinations of flux and resolution are currently available. Given the rich structure seen here for $\mathrm{H}_{2} \mathrm{~S}$, further study of these isoelectronic systems seems warranted. The interpretation of new (and existing) spectra would be greatly facilitated by concurrent measurements on the fully deuterated isotopic species, as was seen here for $\mathrm{H}_{2} \mathrm{~S}$. (Note that the $\mathrm{L}_{2,3}$ spectrum of $\mathrm{SiD}_{4}$ was recently measured with fairly high resolution [2].)

\section{VII.C. Further studies of core-excited electronic states using high- resolution $\mathrm{x}$-rays}




\section{Photoelectron spectroscopy}

Core-shell PhotoElectron Spectroscopy (PES) mainly provides information about the core-excited ion. However, as discussed in several parts of this thesis, the structure of core-excited Rydberg states is similar to that of corresponding core-excited ions, because the Rydberg electron has little influence on the rest of the molecule. With the development of brighter high-resolution x-ray monochromators, e.g. using undulator radiation or rotating $\mathrm{x}$-ray anodes, and electron analyzers with better resolution and throughput, coreshell PES is approaching the fundamental limits of natural linewidths. Such measurements can provide useful complementary information for the interpretation of high-resolution XANES spectra. Several examples have been mentioned: (1) In Chapter III, a recent measurement of the vibrational spacing in carbon-1s-ionized $\mathrm{CO}$ was compared to values for core-excited Rydberg states. (2) In Chapter VI, a comparison of high-resolution XPS (i.e. PES) and Auger electron spectroscopy was cited which predicted the core-level molecular-field splitting observed in the photoionization spectra. (3) Also in Chapter VI, high-resolution photoelectron spectroscopy results were used to predict the lack of observable vibrational structure for the higher $(2 p)^{-1}$ Rydberg states.

There are obvious extensions of the useful results listed here, especially as the experimental capabilites improve. Within the fundamental limitations of natural linewidth, the analysis of any series of core-excited Rydberg states will be facilitated by a detailed knowledge of the geometry and vibrational spacings of the corresponding core-ionized molecules, as measured by high-resolution PES. Core-level splittings due to molecular fields have, in fint, been studied more by PES than by XANES (see references given in Section VI.A). By directly measuring core-level splittings using PES, one may avoid the difficulties encountered in Chapter VI where the core-level splittings are convoluted with the complicated structure of the Rydberg final states. This approach is not yet feasible for deeper core levels, but may soon be within reach. The chemical effect on sulfur $2 p$ natural 
linewidths, demonstrated by the comparison the $\mathrm{SF}_{6}$ and $\mathrm{H}_{2} \mathrm{~S}$ results (Chapters $\mathrm{V}$ and $\mathrm{VI}$ ), is also better to measure $w$ th PES. The natural linewidths measured in XANES for the upper Rydberg states show some dependence upon the nature of the excited electron. This is particularly troublesome if one wishes to compare values for different molecules, because the correspondence of Rydberg states may not be obvious between molecules. It is simpler to use PES, where the main line represents a well-defined final state in each molecule.

\section{Resonance autoionization electron spectroscopy}

This section and the next explore the possibility of studying the neutral core-excited states accessed in XANES by examining the products of the state's decay. For example, the states accessed in the soft $\mathrm{x}$-ray region typically decay by an autoionization (i.e. Auger) process. The angular and energy distribution of the ejected electrons may provide information about the initial state in the decay, which is the final state accessed in XANES. One example was mentioned in Chapter V, where the autoionization spectrum of the (S 2p)${ }^{1} \mathrm{a} 1 \mathrm{~g}$ resonance indicated the inner-shell character of the $\mathrm{a}_{1 \mathrm{~g}}$ orbital through the presence of a "participant" feature. In fact, a number of core-excited valence-shell resonances in various molecules have been studied in this way. As this technique becomes possible with better resolution and statistics, narrower and less-intense resonances can similarly be studied. This could be particularly useful for the study of mixed resonances, i.e. states in which the excited electron has a mixture of Rydberg and valence character. For example, the autoionization spectra for the (C $1 s)^{-1} 3 s-a_{1}$ and $(\mathrm{O} 1 s)^{-1} 3 s-a_{1}$ states in $\mathrm{H}_{2} \mathrm{CO}$ could be compared. The relative intensities of "participant" features would provide a direct mapping of the relative amplitude of the $3 \mathrm{~s}-\mathrm{a}_{1}$ orbital at the carbon and oxygen atoms. This information could be used to test the different possible explanations of the unusual behavior of these resonances (see Section IV.E). 


\section{Photo-fragmentation spectroscopy}

To study the decay of core-excited states, it is also common to examine the ions which are produced. Energy, mass, and angular distribution of ions can be measured, often in coincidence with other ions or photoelectrons. The distribution of ions shows a dependence on the excited state which is decaying, e.g. the products observed from a coreexcited valence-shell resonance may differ from those of a corresponding core-ionized state. These results can be used to infer the spatial distributions of the orbitals accessed in XANES. Rapidly dissociating excited states are particularly well-suited to this type of study, e.g. the branching ratio for different ions produced by 2 p-excited $\mathrm{H}_{2} \mathrm{~S}$ provides direct evidence for the dissociation of the $(2 p)^{-1}$ valence-shell states (see Chapter VI). One possibility, suggested by the results of Chapter IV, is to study the (C 1s $)^{-1} 3 s-a_{1}$ and $(C$ $1 \mathrm{~s})^{-1} 3 \mathrm{~s}-\mathrm{a}_{1}$ resonances in this way. This could confirm the proposed rapid dissociation used to explain the linewidths of these states. 


\section{REFERENCES:}

1. M. O. Krause and J. H. Oliver, J. Phys. Chem. Ref. Data 8, 329 (1979).

2. D. G. J. Sutherland, G. M. Bancroft, J. D. Bozek, and K. H. Tan, Chem. Phys. Lett. 199, 341 (1992). 

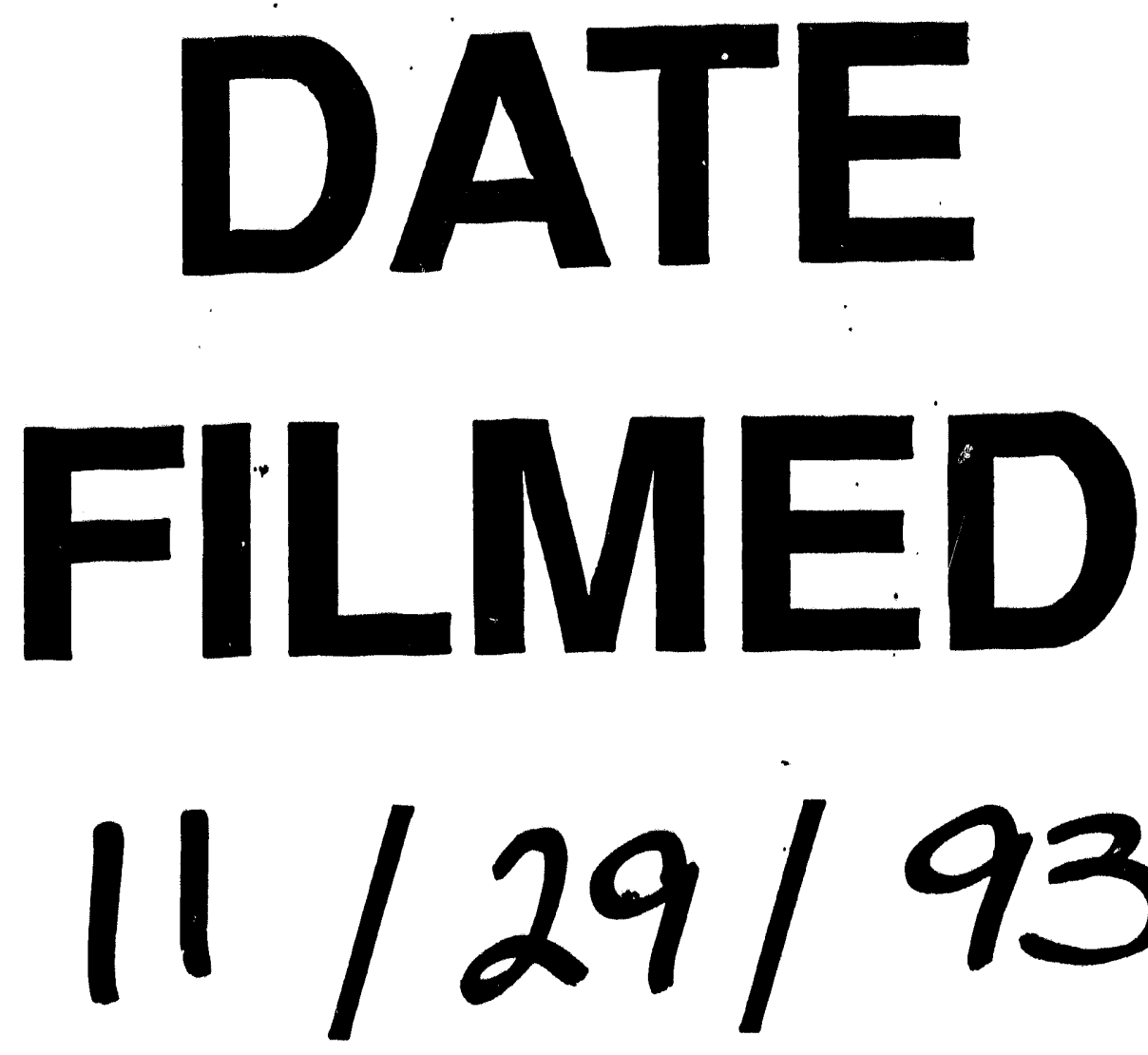

93
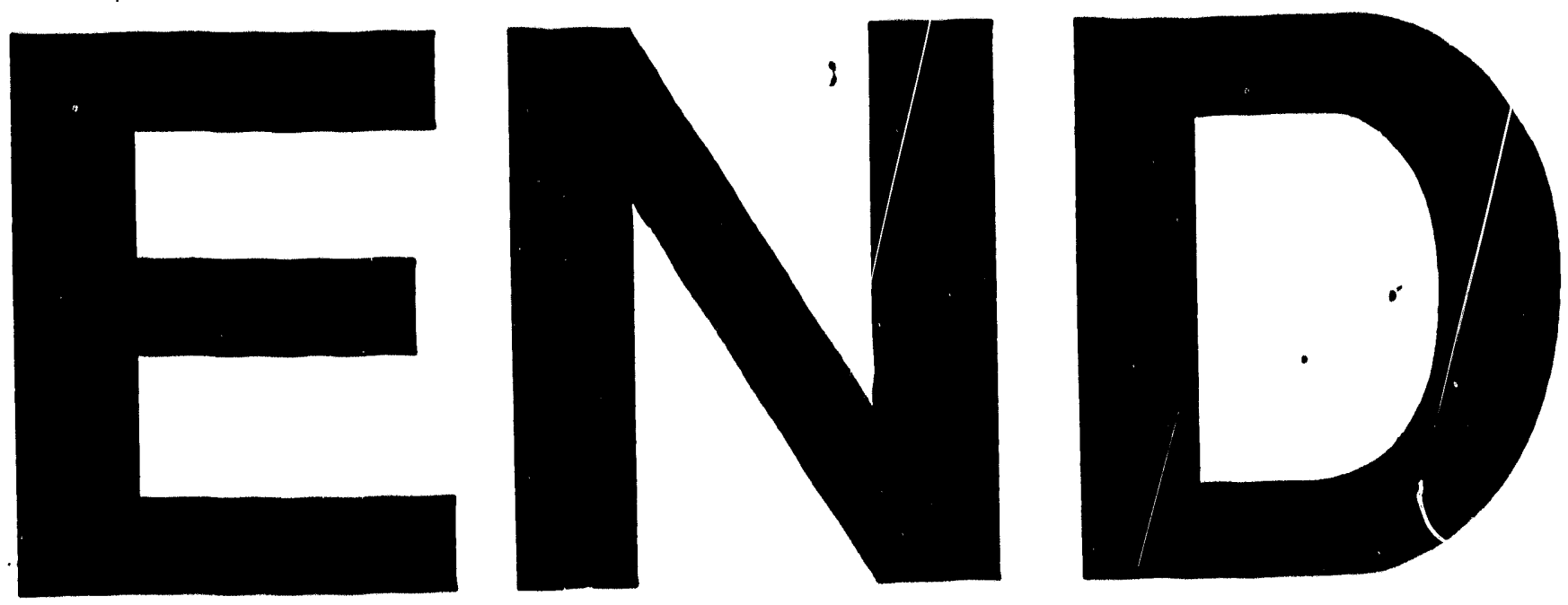
